University of Louisville ThinkIR: The University of Louisville's Institutional Repository

Electronic Theses and Dissertations

$5-2016$

\title{
Mitigating intimate partner violence to promote safe linkage to care for South African women who test HIV+ during mobile counseling and testing.
}

Leslie Lauren Brown

University of Louisville

Follow this and additional works at: https://ir.library.louisville.edu/etd

Part of the Social Work Commons

\section{Recommended Citation}

Brown, Leslie Lauren, "Mitigating intimate partner violence to promote safe linkage to care for South African women who test HIV+ during mobile counseling and testing." (2016). Electronic Theses and Dissertations. Paper 2394.

https://doi.org/10.18297/etd/2394

This Doctoral Dissertation is brought to you for free and open access by ThinkIR: The University of Louisville's Institutional Repository. It has been accepted for inclusion in Electronic Theses and Dissertations by an authorized administrator of ThinkIR: The University of Louisville's Institutional Repository. This title appears here courtesy of the author, who has retained all other copyrights. For more information, please contact thinkir@louisville.edu. 
MITIGATING INTIMATE PARTNER VIOLENCE TO PROMOTE SAFE LINKAGE

TO CARE FOR SOUTH AFRICAN WOMEN WHO TEST HIV+ DURING MOBILE COUNSELING AND TESTING

\author{
By \\ Leslie "Lauren" Brown \\ L.C.S.W., Tennessee Board of Health, 2012 \\ M.S.S.W., University of Tennessee, 2008 \\ B.A., Middle Tennessee State University, 2003
}

\begin{abstract}
A Dissertation Submitted to
the Faculty of the Raymond A. Kent School of Social Work

of University of Louisville

in Partial Fulfillment of the Requirements

for the Degree of

Doctor of Philosophy

In Social Work

Raymond A. Kent School of Social Work

University of Louisville

Louisville, Kentucky

And

University of Kentucky
\end{abstract}

May, 2016 
C2016 by

Leslie Lauren Brown

All rights reserved 

MITIGATING INTIMATE PARTNER VIOLENCE TO PROMOTE SAFE LINKAGE

TO CARE FOR SOUTH AFRICAN WOMEN WHO TEST HIV+ DURING MOBILE COUNSELING AND TESTING

\author{
By Leslie "Lauren" Brown \\ LCSW TN Board of Health, 2012 \\ MSSW University of Tennessee, 2008 \\ BA Middle Tennessee State University, 2003
}

A dissertation approved on

April 11, 2016

By the following Dissertation Committee:

Professor and Associate Dean of Research, Adriaan Michiel van Zyl

Professor and Distinguished University Scholar, Anita Barbee

Professor and Doctoral Program Director, Bibhuti Sar

Assistant Professor, Emma Sterrett-Hong

Associate Professor, Marie Antoinette Soss 


\section{DEDICATION}

Dedicated to all women who have lost their lives or voice while enduring intimate partner violence, especially those living with HIV who have had to fight the hardest for survival.

Dedicated to the women who taught me to use my voice to help others, my mother, Margaret Lutz Brown, and grandmothers, Lottie Marie Spencer and Joyce Ann Lutz.

Dedicated to my daughter, Zollie Maude Sewell-Brown, whom I will raise to exercise her voice as an unwavering instrument to help women throughout the world. 


\section{ACKNOWLEDGEMENTS}

It is difficult to succinctly list all the ways Dr. Riaan van Zyl has been an exemplary mentor to me. The gravity of his efforts and acts of support far outweigh anything I could write about him and leave me with an indelible sense of gratitude. Dr. van Zyl is a consummate professional: He has consistently modeled professional boundaries while maintaining kindness and poise; displayed patience while also pushing me to try harder; demonstrated punctuality while never hurrying me along; and offered necessary critique of my work while also encouragement and optimism. He is a man of his word who so often accomplishes his word with impressive pithiness. He is a solid listener who only talks when there is something real to be spoken. Because of his support I had the privilege to travel to South Africa, where a cadre of his friends and family pampered my family and me with love, laughter and adventure. From my first semester in the doctoral program where we connected over a shared love for Tolstoy, to the trenches of an international study and its entire convolution, Dr. van Zyl has shown he is more than just a person who cares about my scholarship but also is someone who cares about my experience and well-being along the path of scholarship. Although he is an expert in his field, he has always 
placed full faith in my ability to contribute to his life's work. His model of instruction, while more difficult than some, allowed me to grow as an independent and critical thinker, learning lessons that changed the very core of my intellectual self. He truly represents the essence of what it is to be a Social Work mentor.

My respect and appreciation for Dr. Anita Barbee is far reaching and everexpanding. From one dynamo to another, she inspires that academics can be humble, funny, and creative and still get things done with great integrity. She makes even the hardest work look effortless and fun! Her love for my daughter and compassion for me as a mom remind me how strong women can be when they work together. There is not another soul like her, and her encouragement has been priceless.

I am incredibly grateful to Dr. Bibhuti Sar who saw me fit for the doctoral program, cheered me on when I became mentally and physically fatigued, strengthened my writing by admonishing my use of too many quotes, and modeled how to gracefully respond to moody teenage-like students grappling with the ups and downs of the PhD process. I can only hope to be a sliver as patient and kind as he is consistently.

I owe a great deal of gratitude to Drs. Marie Antoinette Sosou and Emma Sterrett-Hong who both so willingly supported my study and gave up their time to serve on my committee. 
I would also like to thank Norma Kyriss for always having answers and my back along this path; Jessica Acuña for sacrificing so much of her time to help with the study's implementation (...for she gave up her only begotton i-pod); for Lyle Cooper for providing unyielding encouragement to me and leading me to Kent school in the first place; for Jennifer Lawson for endlessly meeting for coffee so we could talk about how busy we were; for the staff of Shout-it-Now for all of their time and effort in mobilizing this study, with particular gratitude to Kathryn Pahl who fought for the study because she believes women living with HIV need greater care; for the Research team who administered this study and their extreme diligence in exchange for humble pay: Rejoice, Mona, Zimkhitha, \& Yandisa.

I could not have finished this book without the steadfast support of my partner, Jon Sewell, who gave up many hours of his life so I could work at the big desk upstairs, and who will sacrifice his mustache as my hard-earned reward; my mom for burning up the road between Kentucky and Tennessee so that she could help with Zollie, clean our kitchen, cook us food and lavish us with unconditional love and laughter; my aunt Linda Turley and Elizabeth Cato, who both have supported all my radical endeavors and helped me tremendously during times of their own great difficulty; my "little" brother, Wes(t) Brown, for being patient with me while I have been a mere ghost of the support I normally am and for loving me for who I am; my dad, Robin Brown, for teaching me to put 
the pedal to the medal and push forward with eyes open and all my might; and my other brother, Christopher R. Brown, for modeling courage and strength also in the face of great difficulty.

I would like to acknowledge those who were lost along the way of this process. My father-in-law, Pat Patrick, who was my father-daughter duet partner and a dear friend greatly missed; my cousin Gabe, who inspired me by showing what it means to put love forward in this world and who left this world far, far too soon; and my uncle Steve Dunn, who would have been a master provocateur during this time of great political upheaval.

I would like to thank my best friends who have had to idly endure an increasingly more selfish version of me for which was necessitated in order to meet the demands of the doctoral student lifestyle. To you I hope to be returned in full joy and laughter, knowing I am indebted in all the emotional currency you have given me for which I have so infrequently been able to reciprocate along this journey. I never forget an owed debt.

Finally, I would like to thank the University of Louisville's Commission on Diversity and Racial Equality for their financial support. Because of their funding, significantly more women were able to reap the rewards of the HIV IPV Risk and Safety protocol. 


\title{
ABSTRACT
}

\section{MITIGATING INTIMATE PARTNER VIOLENCE TO PROMOTE SAFE LINKAGE TO CARE FOR SOUTH AFRICAN WOMEN WHO TEST HIV+ DURING MOBILE COUNSELING AND TESTING}

\author{
Leslie "Lauren" Brown
}

April 11, 2016

Sources warn the global HIVIAIDS epidemic will not be halted without significant reductions in Intimate partner violence (IPV). South African women have been hardest hit by the HIV-IPV link, suffering deleterious health outcomes as sequelae of significant barriers to HIV care. Extant literature focuses on HIV prevention, and there is a paucity of effective methods for mitigating IPV-related barriers among women living with HIV.

This study was a Phase II trial testing the effectiveness and safety of the HIV IPV 
Risk and Safety (HIRS) protocol administered to women experiencing IPV and testing HIV+ during mobile HIV Counseling and Testing (HCT). Research questions compared experiences between a Standard of Care and an Experimental group with two dosage levels. All participants $(N=255)$ were black South African females residing in Gauteng province and aged about 33 years $(M=33)$. Two psychometric instruments were created for this study: the Danger Indicator and the HIRS scale with the latter having good reliability (Cronbach alpha .882 ) but both needing refinement in validity.

The protocol was shown to be effective in mitigating violence upon partner notification of serostatus $\left(X^{2}{ }_{(1)}=7.83, p=.005\right)$ and promoting linkage to care, with participants aged 33-43 years trending towards significant $\left(X_{(1)}^{2}=3.57\right.$, $p=.059)$. The protocol was found to be feasible to implement, having impressive retention, brevity in administration, and high usage of safety strategies with minimal risks resulting from participation. Satisfaction was confirmed through positive participant ratings and safety through both non-significant group differences on the HIRS scale $(t(1,237)=-1.09 ; p=.278)$ and the perceived benefits of study participation far outweighing harms.

Women receiving the protocol displayed notable self-determination towards selfprotection and suffered significantly less re-assault upon partner notification. The number of participants utilizing certain safety strategies suggests this brief intervention was effective in galvanizing South African women to reclaim power 
and act towards the betterment of their health.

Findings herald the promise of a protocol that effectively mitigates IPV to improve HIV outcomes. The effectiveness of the HIRS protocol, in facilitating safe partner notification of serostatus, could be a significant contribution to the knowledge base, but more research is needed to confirm these results. 


\section{TABLE OF CONTENTS}

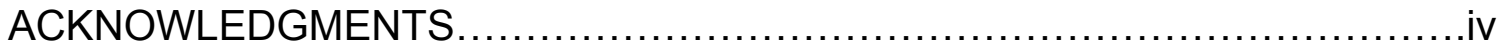

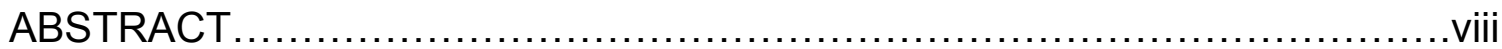

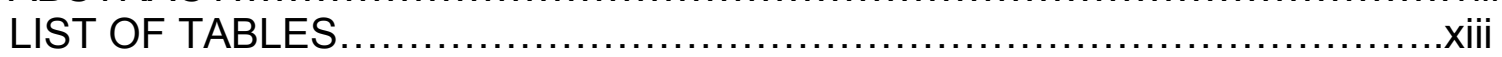

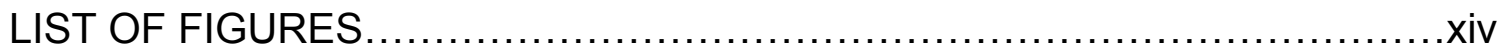

CHAPTER 1. INTRODUCTION: THE HIV-IPV INTERSECTION $\ldots \ldots \ldots \ldots \ldots \ldots . . \ldots$

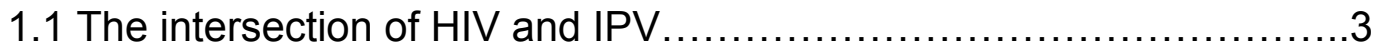

1.2 IPV as a Barrier to HIV Care \& Health-related Implications..............10

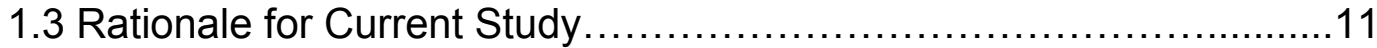

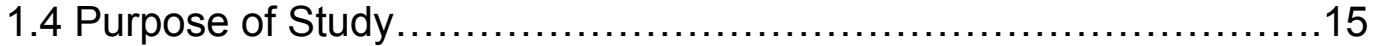

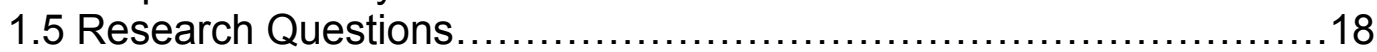

1.6 Summary \& What to Expect....................................20

CHAPTER 2. LITERATURE REVIEW \& THEORETICAL BACKGROUND.....22

2.1 Empirical Support for the Standard of Care .............................24

2.1.1 Theoretical Background of Standard of Care .......................26

2.2 Empirical Support for Experimental Components of HIRS Protocol...30

2.2.1 Theoretical Background of HIRS Protocol...................42

2.3 Evaluating the Intervention \& the Implementation Process............51

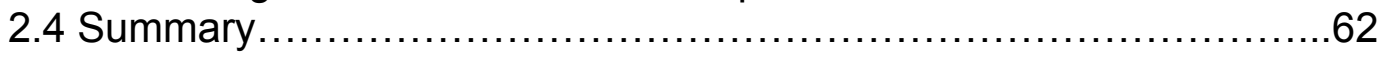

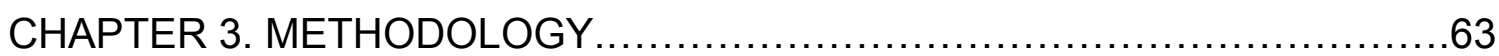

3.1 Research Questions \& Hypotheses..............................64

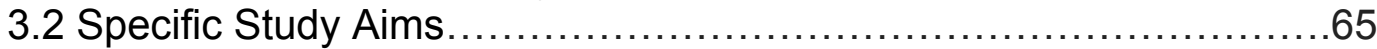

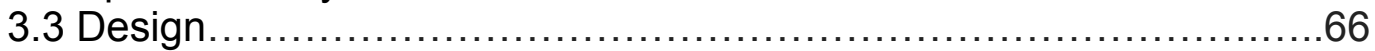

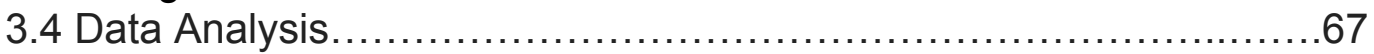

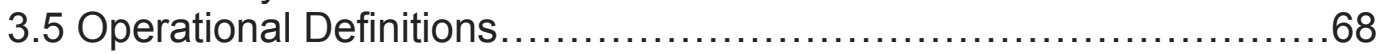

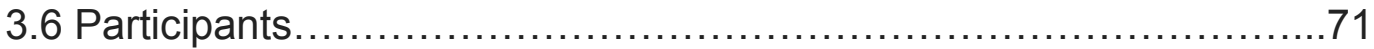

3.7 Description of Instruments \& Rationale for Use.......................73

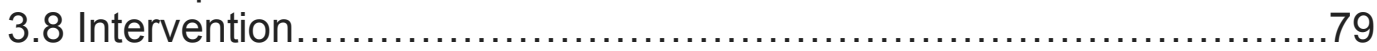

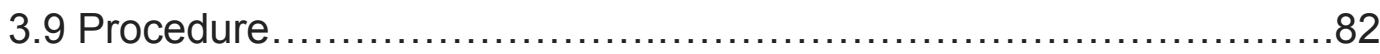

3.10 Ethical considerations.......................................90

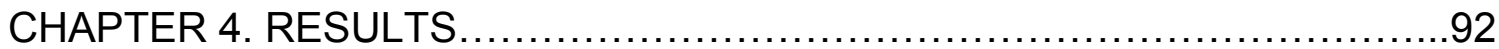

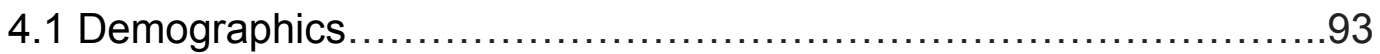

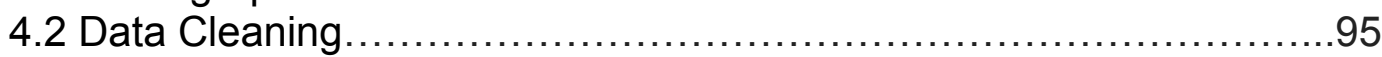

4.3 Reliability and Validity Testing of Instruments......................97

4.4 Conceptual Definitions............................................99

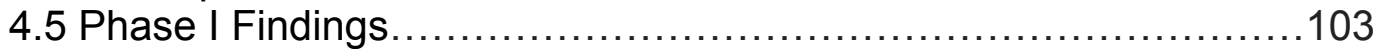




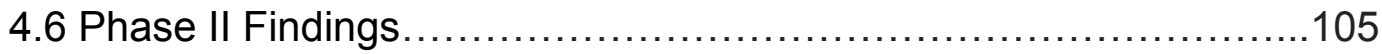

4.7 Primary Research Question 1A.................................. 107

4.8 Primary Research Question 1B...................................108

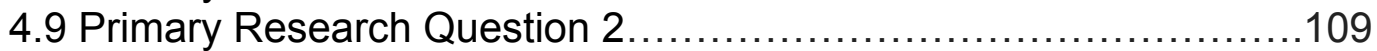

4.10 Secondary Research Question 3A..............................113

4.11 Secondary Research Question 3B..............................114

4.12 Additional Analyses................................................

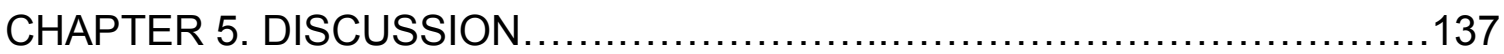

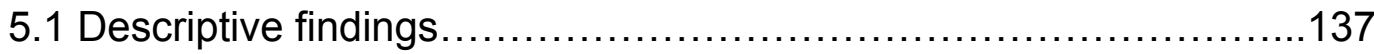

5.2 Demographic Factors................................................ 142

5.3 Contribution of Psychometric Instruments.......................... 143

5.4 Research Question 1A................................................ 145

5.5 Research Question 1B .......................................... 151

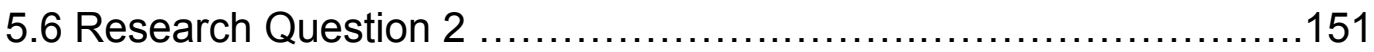

5.7 Research Question 3A ........................................ 154

5.8 Research Question 3B ............................................

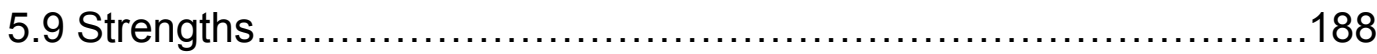

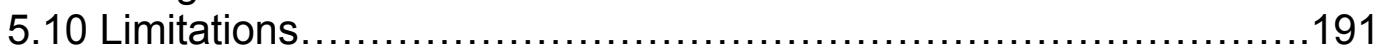

5.11 Implications for Future Practice.................................. 196

5.12 Recommendations for Future Research...........................198

5.13 Conclusion..........................................................

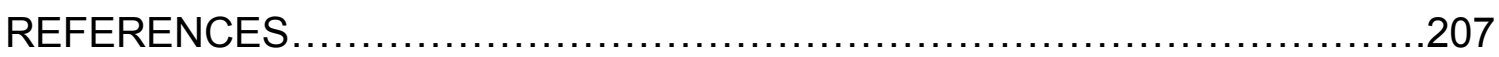

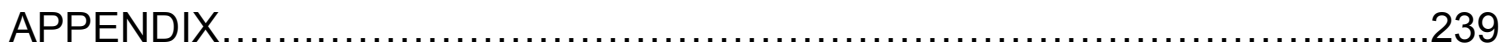

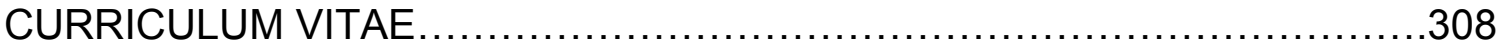




\section{LIST OF TABLES}

Table 3.1

Table 4.1

Table 4.2

Table 4.3

Table 4.4

Table 4.5

Table 4.6

Table 4.7

Table 4.8

Table 4.9

Table 4.10

Table 4.11

Table 4.12

Table 4.13
Instruments \& Program Components.........................73

HIRS Pre/Post \& Mean Difference Scores I................108

HIRS Pre/Post \& Mean Difference Scores II.................109

Linkage to Care.............................................110

Linkage to Care by Age Group............................112

Satisfaction Responses I...............................113

Satisfaction Responses II..........................................115

Acceptability Responses................................116

Feasibility Responses......................................118

Frequency of Strategies Used..........................122

Forms of IPV .............................................. 124

Danger Score Comparisons.................................129

Tester Status ...............................................133

Past Tester Diagnosis Dates.............................133 


\section{LIST OF FIGURES}

Figure 1.1

Figure 1.2

Figure 1.3

Figure 1.4

Figure 2.1

Figure 2.2

Figure 3.1

Figure 4.1

Figure 4.2

Figure 4.3

Figure 4.4

Figure 4.5

Figure 4.6

Figure 4.7

Figure 4.8

Figure 4.9

Figure 4.10

Figure 4.11

Figure 4.12

Figure 4.13
HIV Care Continuum.

.2

Pathways from Gender Inequality to Increased HIV Risk among Survivors of Violence. .6

Model of the Dynamics of HIVIAIDS Stigma....................9

Causes and Consequences of Violence against Women

Living with HIV. 11

HIVIAIDS Power and Control Wheel

Contextualized Assessment for Strategic Safety

Planning with Battered Women................................51

Research Design...........................................67

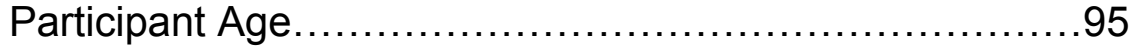

Consort Diagram............................................ 97

Linked to Care by Post-test..................................110

Linked to Care within 30 Days..............................111

Number of Safety Strategies Used.......................120

Frequency of Strategies Used............................121

Non-violent control..........................................125

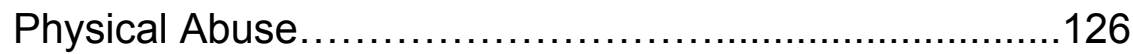

Sexual Abuse........................................... 127

Danger Indicator Scores.................................129

Pre-test Perceived Safety in Getting to Medical

Appointments 131

Post-test Perceived Safety in Getting to Medical

Appointments 132

Tester Status Distribution.

132 


\section{CHAPTER 1}

\section{INTRODUCTION: THE HIV-IPV INTERSECTION}

The story of the human immunodeficiency virus (HIV) epidemic in South Africa is one of widespread devastation and yet also great hope. Although the country has been hardest hit by the HIV epidemic (UNAIDS, 2012), South Africa has one of the strongest national campaigns for HIVIAIDS relief (UNAIDS, 2015b). Additionally, countries worldwide have become allies to tackle the epidemic in sub-Saharan Africa, with greatest force in South Africa. From these efforts have abounded stories and statistics of life-saving programs and initiatives. Yet, for many, HIV continues to claim their lives at an alarming rate, and the number of those impacted in South Africa remains formidable (Mall, Middlekoop, Mark, Wood \& Bekker, 2013). Despite the rollout of more testing and treatment services, many individuals continue to be unaware of their status, and the majority of individuals who have been tested are not utilizing available treatment (Mall et al; UNAIDS, 2000). Given the investment in curbing the disease in South Africa, the need for evidence-based programs that provide preventative measures and halt disease progression is ever increasing, and there is a particular urgency for the development of interventions that effectively 
reach those who do not access services offered in traditional mediums (Krazner et al., 2010).

The HIV care continuum (Figure 1.1) is a model that illustrates the stages of care needed in order for a person with HIV to receive the most long-term health benefits (AIDS.gov, 2015). Approaches like HIV Counseling and Testing (HCT) initiate the care process by informing individuals of their HIV diagnosis. $\mathrm{HCT}$ is an important technique used during the diagnostic stage because it has been associated with reductions in risky behaviors (Painter, 2001), and, consequently, lowered transmission rates (Beer et al., 2012). Further, it can be instrumental in encouraging individuals to successfully engage in the subsequent steps in the care continuum.

\section{Figure 1.1 HIV Care Continuum}

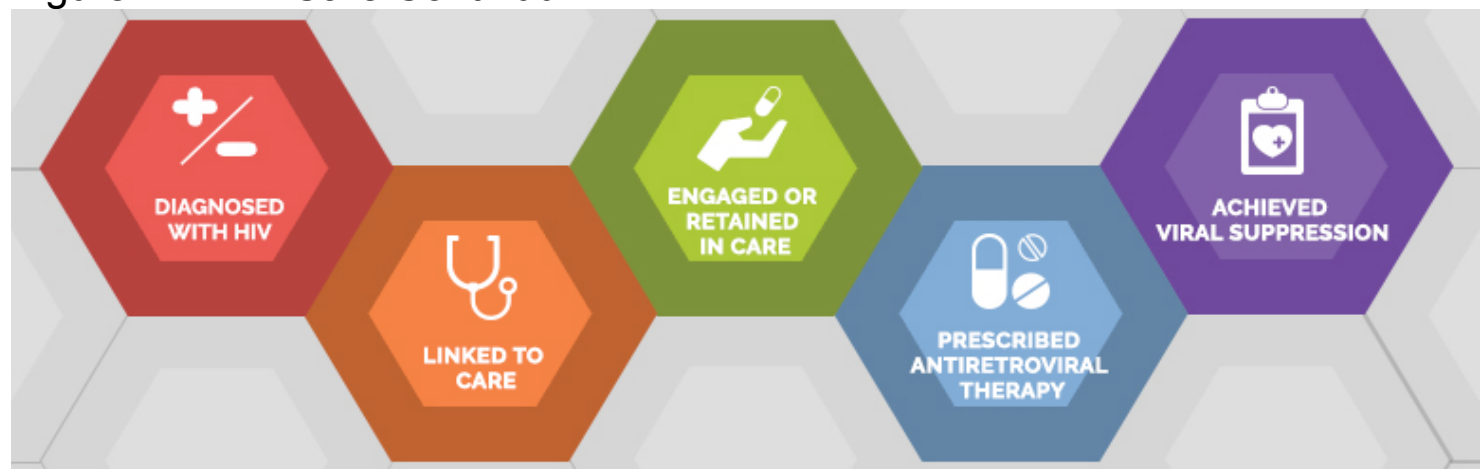

Figure 1.1 HIV Care Continuum AIDS.gov (2015). HIV care continuum. Retrieved on February 6 , 2016 from https://www.aids.gov/federal-resources/policies/care-continuum/

Thus, the effectiveness of HCT can determine if an individual begins the care continuum or falls off the treatment cascade, either delaying or abstaining from linking to care. Linkage to care is the next important stage in the continuum because the care a person receives helps assess disease progression: eligibility 
for antiretroviral therapy (ART) is based on CD4 cell count and often linking to some form of care is the only way to receive these results. Unfortunately, a vast number of individuals delay ART initiation and suffer health consequences such as opportunistic infections and consequently death (Van Zyl, Brown \& Pahl, 2015).

Past research has helped refine HCT methods so that programs more effectively encourage a greater number of individuals to link to care and at a faster pace. Although it is difficult to find an average linkage rate in the literature due to variations in collection periods and classifications of "successful" linkage periods, rates ranged from only $10 \%$ (Bassett, Regan et al., 2014) to $47 \%$ (Losina et al., 2010), 53.4\% (Krazner et al., 2010) and 51\% in a large sample of

South African youth (Van Zyl et al., 2015). While most of these numbers indicate mobile HCT can be effective in assisting many, the demand for more improvements with HCT is underscored. Additional research is required to identify and reduce barriers to timely linkage to care. Notably, the improvement and expansion of services like HCT has been recognized as vital to AIDS eradication (Centers for Disease Control \& Prevention (CDCa, 2015, pg. 1).

\subsection{The intersection of HIV and IPV}

Global strategies to end the HIV epidemic have also identified various societal injustices that contribute to disease transmission and progression and act as barriers to available services such as HCT and ART. Successful eradication of the epidemic hinges on advances of an array of human rights' 
issues (UNAIDS, 2015a), and one issue that is a fulcrum of the HIV epidemic is that of gender inequality (UNAIDS, 2012). Almost $60 \%$ of HIV cases worldwide are among women in sub-Saharan Africa (UNAIDS, 2012), and the disease is the primary cause of death among those of reproductive age (AVERT, 2015). In South Africa alone, there are more than 3.5 million women living with HIV. Young women (aged 20-24) are four times more likely to become HIV infected than their male counterparts, and women (aged 30-34) experience the highest prevalence rate of $42.6 \%$ (UNAIDS, 2012).

It is believed that women suffer disparately from the HIV epidemic, in large part, due to gender inequalities. One mechanism to keep women in their place is gender-based violence (GBV). The elimination of GBV was listed as a top ten goal for the 2015 plan to eradicate HIV (UNAIDS, 2013b); thus, the connection between HIV and GBV has already been made. This elimination plan is paramount for the health and well-being of South African women considering the country has simultaneously been ravaged by the epidemic of gender-based violence and the highest documented rate of femicide in the world (Wong, Huang, DiGangi, Thompson \& Smith, 2008). While GBV may be perpetrated in many different ways by all sorts of men who encounter women publically and privately, the private form of GBV_-intimate partner violence (IPV)—is the most pervasive globally (Maman, Mwambo, Hogan, Kilonzo, \& Sweat, 2002) and is used as a proxy for GBV (UNAIDS, 2015a).

IPV afflicts women gravely in HIV prevalent areas such as South Africa. Definitions vary, but a basic description of IPV includes "physical, sexual or 
psychological harm by a current or former partner or spouse" (CDCb, 2015, para. 1). Prevalence rates also vary; one in four women (Bendall, 2010), or up to $55 \%$ (Dunkle et al. as cited in Adams et al., 2011) of women in South Africa reportedly have experienced IPV at some point in their lives. The prevalence is thought to be higher in actuality with these rates being non-reflective of the gross underreporting of IPV that occurs (Boonzaier \& Gordon, 2015). Many view IPV as a private matter, with some women opting, for myriad reasons, to not discuss what they have endured (Bendall, 2010). Others contextualize IPV in South Africa as a cultural matter-both a vestige of post-apartheid culture, where IPV stems from the general overuse of violence as a common resolution tactic (Jewkes, Levin \& Penn-Kekana, 2002) and an ideology of gender-based violence propagated as a normal, acceptable or even desired mechanism for maintaining traditional masculinity (Jewkes et al. as cited in Boonzaier \& Gordon, 2015). Hence, resulting from cultural norms steeped in gender-based violence, a certain level of IPV is seen as acceptable (Jewkes et al., 2002).

In addition to the baseline gender-based violence commonly endured in South Africa, women living with HIV experience increased IPV through a number of different mechanisms. It is important to note that often an HIV diagnosis does not alone create violence in a relationship (although some violence has been reported as occurring after an HIV diagnosis only, and this is reviewed in Chapter Five). Rather, many women with HIV diagnoses have already been experiencing IPV in their relationship. Thus, the pathway between HIV and IPV is multidirectional (see figure 1.2); IPV increases the risk for HIV transmission, and 
living with HIV increases the risk for [further] IPV in various forms (Ball, 2013;

Dunkle et al. 2004; Hale \& Vazquez, 2011; Jewkes, Sikweyiya, Morrell, \& Dunkle, 2011; Maman, Yamanis, Kouyoumdjian, Watt, \& Mbwambo, 2010; Prowse, Logue, Fantasia, \& Sutherland, 2013; CDC, 2014; Ramachandran, Yonas, Silvestre, \& Burke, 2010; Ball, 2013; Shi, Kouyoumdjian, \& Dushoff, 2013). Women who are HIV+, compared with their HIV-counterparts, are more likely to report a history of IPV—physical, sexual, a combination of both, (Maman et al., 2002) or psychological (controlling partners) (Dunkle et al., 2004; Hale \& Vazquez, 2011). One theory is that men who batter are more likely to be infected with HIV and transmit infections through forced sexual activity (Dunkle et al.). Physical or sexual IPV can lead to HIV transmission by compromising a person's immune system (Shai, Jewkes, Nduna, \& Dunkle, 2012) or through direct contact if a woman's genitalia are damaged in a sexual assault (UNAIDS, 2013b). Other studies have shown a correlation between HIV transmission and other risk factors related to IPV (Shai et al.). For example, coercion in a relationship can reduce a woman's ability to protect herself (Maman et al.; Shi et al.; Dunkle \& Decker, 2012; Prowse et al., 2013), or negotiate safer sex (Shi et al.; UNAIDS, $2013 b)$ as it may not be safe for her to discuss condom use (Dunkle \& Decker). 
Figure 1.2 Pathways from Gender Inequality to Increased HIV Risk among Survivors of Violence

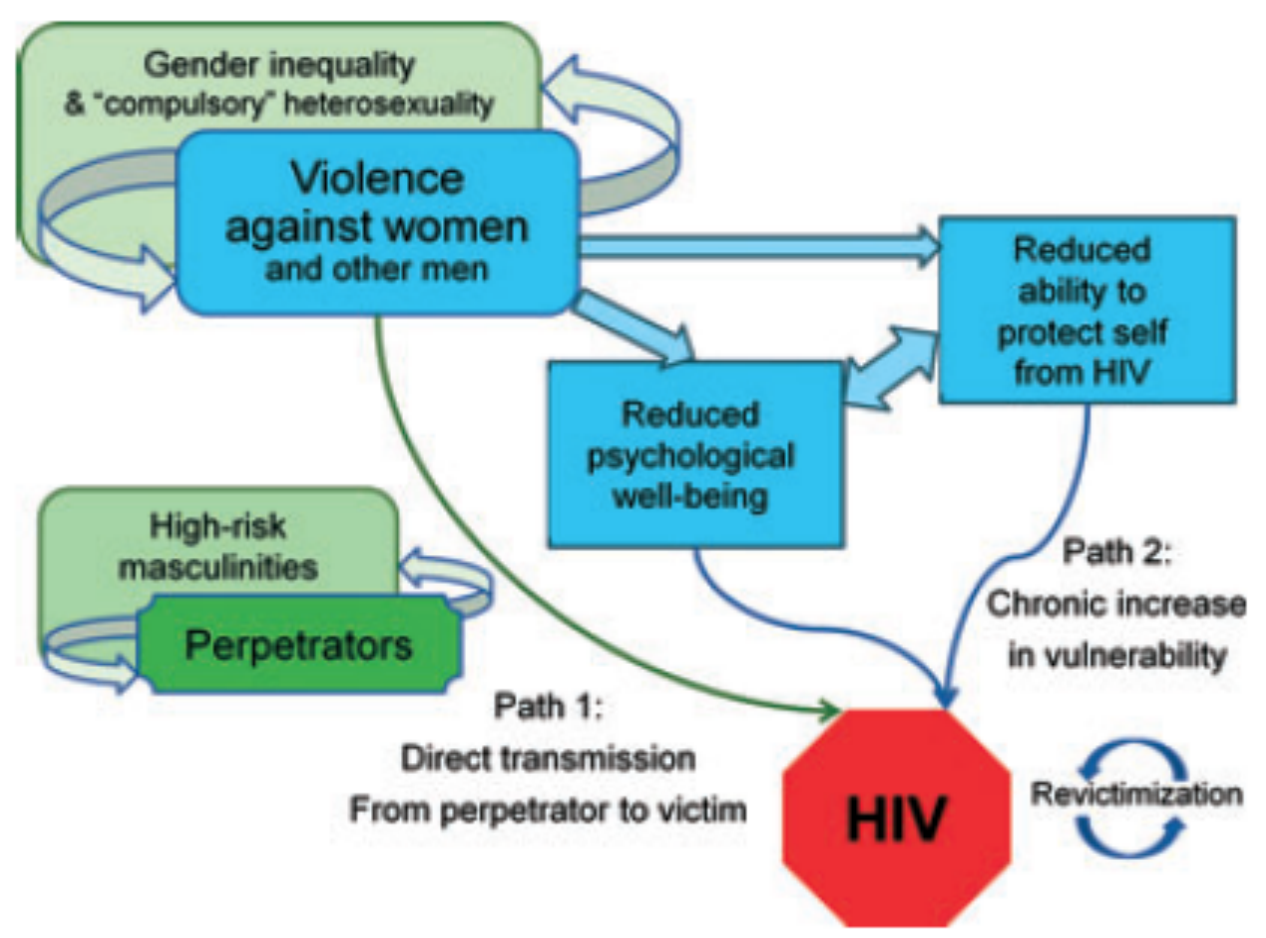

Figure 1.2 "Pathways from gender inequality...to increased HIV risk among survivors of violence." Dunkle, K.L. \& Decker, M.R. (2012). Gender-Based violence and HIV: reviewing the evidence for links and causal pathways in the general population and higher-risk groups. American Journal of Reproductive Immunology, 69(1), 21.

Regardless of the mode of transmission, women living with HIV are more likely to already be experiencing IPV and the diagnosis has been shown to foment further aggression (Prowse et al., 2013). Cultural and structural violence is experienced by women living with HIV, where women are often blamed for the IPV and then pressured by family and society to stay in the relationship. Financial constraints additionally prevent women from having the means to flee the relationship (Hale \& Vazquez, 2011). Personal violence also occurs related to the general cycle of escalating violence in certain types of IPV relationships (Gielen, McDonnell, Burke, \& O'Campo, 2000; WHO, 2012), and upon disclosure of an 
HIV+ serostatus to one's partner (Prowse et al., 2013; Mkandawire-Vahlmu, Stevens, Kako \& Dressel, 2013), often due to the social stigma of the diagnosis (Myer, Rebe \& Morroni, 2007). An exploratory study in sub-Saharan Africa cited that IPV against women with HIV frequently follows the scenario of a man coming home drunk and becoming enraged when either the woman notifies him of her seropositive status or she refuses to have sex with him (Murray et al. as cited in Hale \& Vazquez, 2011). Another study in the area found that $19 \%$ of women reportedly experienced IPV "because of their HIV status," and slightly higher rates were found in the United States, specifically linking physical violence as a result of a woman's serostatus (Nilo as cited in Hale \& Vazquez, 2011, pg. 18). Other women have reported experiencing abandonment or blame by their partner also as a result of serostatus notification (Mkandawire-Vahlmu et al., 2013).

The social stigma of an HIV diagnosis plays a nefarious role in increasing IPV among women living with HIV. Stigma is a form of "labeling or 'othering' of persons or a group of people" (Goffman as cited in Abdool Karim et al., 2013, pg. 352). When someone is diagnosed as HIV+ they often experience a great deal of stigma from their community and in other social realms, and generally this stigma acts as a force that reinforces gender inequalities by reproducing dynamics of power and control (Abdool Karim et al., 2013). HIV-stigma-related abuse is "often aimed at rejecting, dehumanizing, and isolating" people living with HIV (Dlamini et al., 2008, pg. 395).

Some examples of stigma-related verbal abuse towards people living with HIV in African countries are name-calling, the shouting of insults and blame, 
being labeled as an other, and partners seeking retribution or revenge (Dlamini et al., 2008). More, physical abuse has been shown to be related to partner notification of serostatus with partners reacting violently to the shock of the news or becoming enraged at the partner for possibly exposing them to the disease (Dlamini et al., 2008). See figure 1.3 for a description of the model of HIVIAIDS related stigma experienced in many African cultures. In addition to these types of stigma people living with HIV experience, in general, women with IPV are at an increased risk for stigma-related abuse by their partners, and this places a significant hindrance on their ability to engage in HIV-related services, from testing to treatment (UNAIDS, 2013b).

Figure 1.3 Model of the Dynamics of HIVIAIDS Stigma Model of the Dynamics of HIV/AIDS Stigma

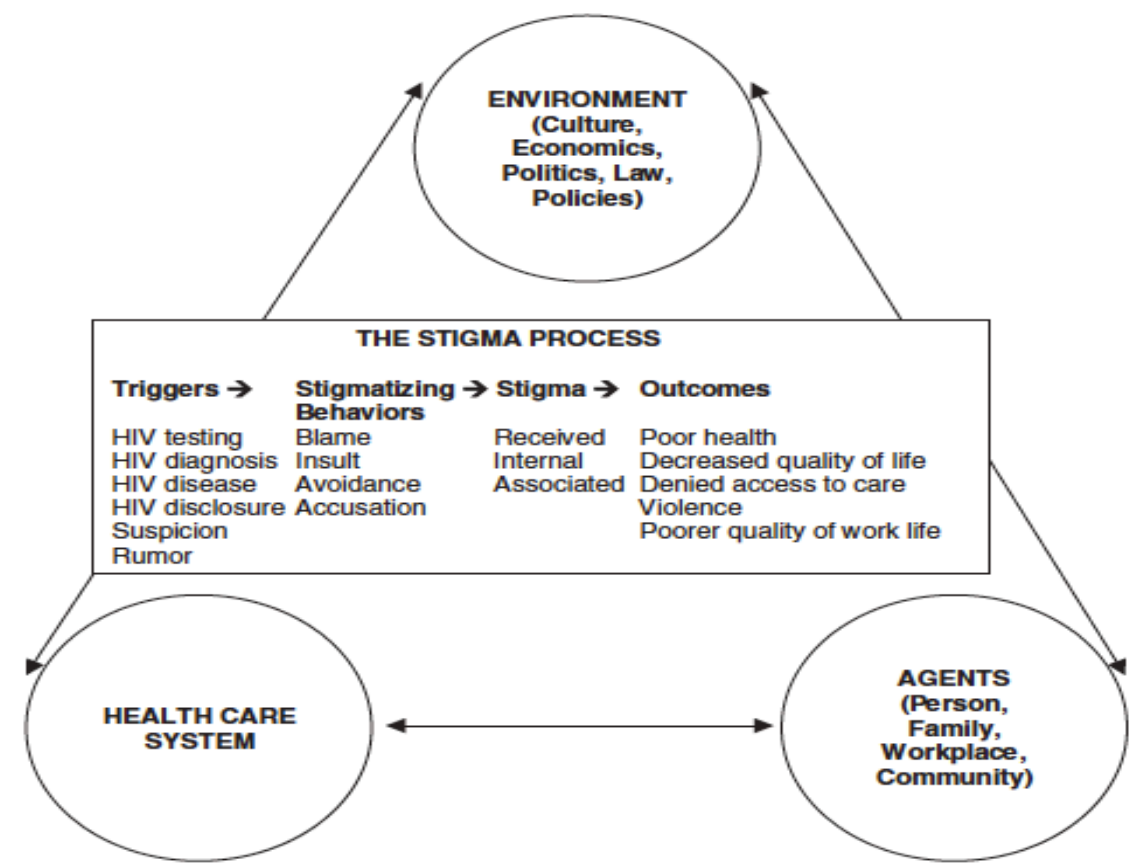

Figure 1.3 Model of the Dynamics of HIVIAIDS Stigma. Dlamini, P.S., Kohi, T.W., Uys, L.R., Phetlhu, R.D., Chirwa, M.L., Naidoo, J.R., Holzemer, W.L., Greef, M., Makoae, L.N. (2008). Verbal and physical abuse and neglect as manifestations of HIVIAIDS stigma in five African countries. Public Health Nursing, 24(5), 392. 


\subsection{IPV as a Barrier to HIV Care \& Health-related Implications}

South African women affected by the HIV-IPV link face insidious barriers to receiving care, and access to HIV testing and treatment remains insufficient among this group (AVERT, 2015). The majority of individuals who receive HIV testing either face subsequent delays to entry to care or are lost to follow-up at some point in the HIV treatment continuum (Christopulous et al, 2013). A portion of this attrition is attributed to the HIV-IPV link (Abramsky et al., 2012; Adams et al., 2011; CDC, 2014; Mkandawire-Vahlmu et al. 2013; Prowse, Logue, Fantasia, \& Sutherland 2013; WHO, n.d), as women with both HIV and IPV face one of the heaviest burdens of care (Hale \& Vazquez, 2011). One study found $16-51 \%$ of individuals reported fear of IPV as a substantial barrier to HIV disclosure (Medley, Garcia-Moreno, McGill \& Maman, 2004; WHO, n.d.). For these reasons, and others, some women may choose to avoid HIV testing from the onset (CDC, 2014, Abramsky et al.), or refrain from linking to care or adhering to treatment once diagnosed (Snyder et al., 2014; Mkandawire-Vahlmu et al.).

Hence, the effects of the HIV-IPV link on South African women's health have been immense with those already HIV+ being at risk of worse health outcomes and conditions (Shafer et al., 2012). Women living with HIV and IPV have been shown to have lower CD4 cell counts (Shafer et al.) and often miss doses of ART when the abuse thwarts their ability to access or adhere to treatments. With the latter case, these women frequently experience blame from healthcare workers for the poor adherence (Human Rights Watch as cited in Hale \& Vazquez, 2011). See Figure 1.4 for the causes and consequences of 
violence against women living with HIV.

Figure 1.4 Causes \& Consequences of Violence Against Women Living with HIV

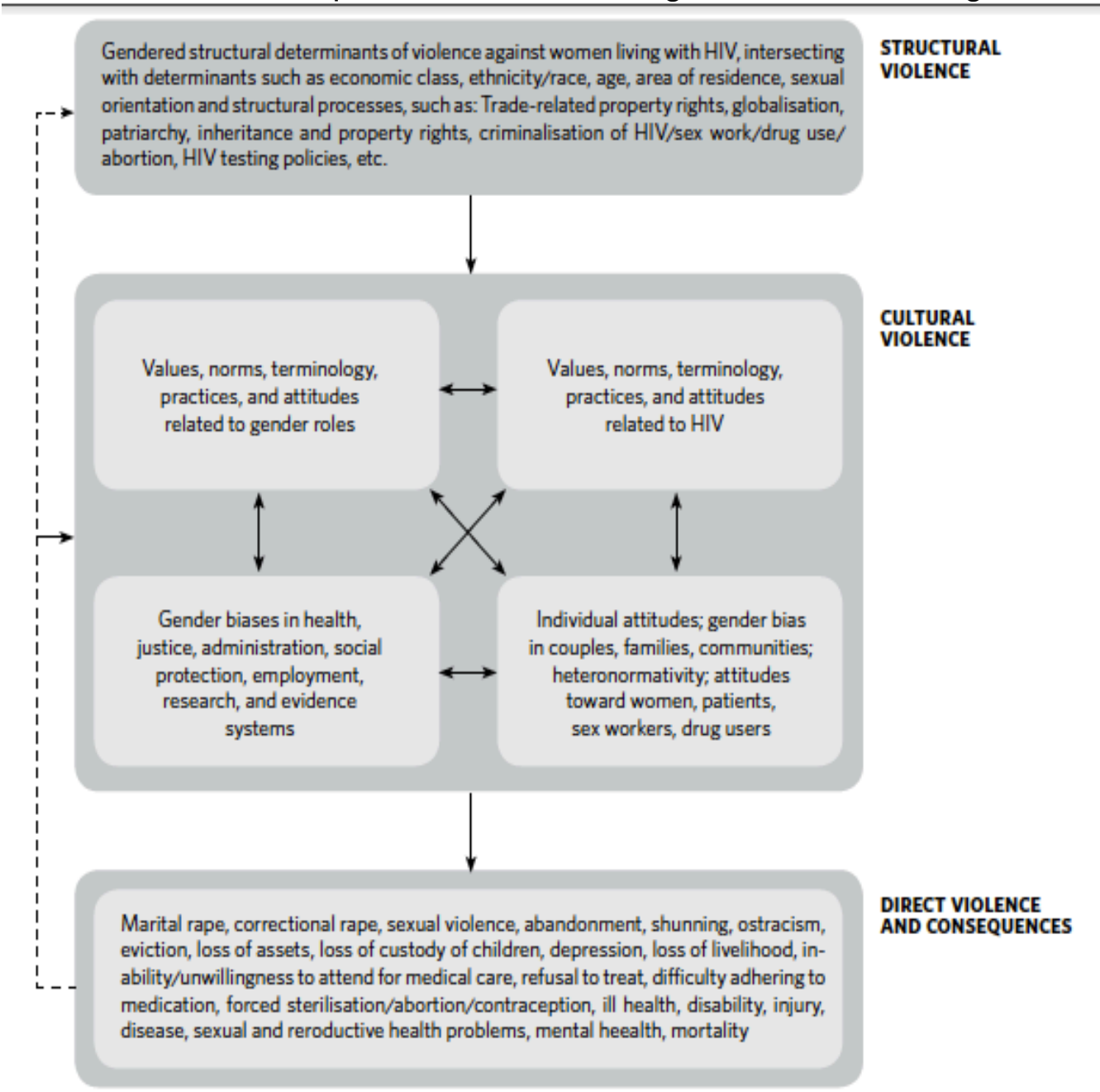

Figure 1.4 Causes and Consequences of Violence Against Women Living with HIV Hale, F. \& Vazquez, M. (2011). Violence against women living with HIVIAIDS: A background paper.

Development Connections. USA, 16.

\subsection{Rationale for Current Study}

The conjoined epidemics of HIV and IPV have ravaged the lives of South African women at a disproportionate magnitude, and the need for effective interventions with a joint target has gained increasing recognition (Abramsky et al., 2012). Much of extant initiatives and research targeting the HIV-IPV 
intersection have focused on addressing IPV as a means for preventing HIV, and this has led to a gap in knowledge as well as supportive services for those who are already living with HIV and experiencing IPV. Unfortunately, there has been little effort globally to mitigate IPV among women with HIV: there is a paucity of empirical support for services effective in diverse settings and populations and a general dearth "of systematic research on this issue" with past research failing to recognize IPV as a consequence of an HIV diagnosis (Hale \& Vazquez, 2011, pg. 4).

This gap emphasizes the great need for initiatives and research that concentrate on women living with HIV and IPV, especially bearing the pervasiveness of IPV among those who attend for social services (Lindhorst, Nurius \& Macy, 2005) and the potential for identifying those "at risk for abuse after an HIV-positive diagnosis" (Gielen, McDonnell, Burke \& O'Campo, 2000, pg. 11). It is, therefore, crucial that providers of HIV testing incorporate IPV assessment into HIV post-test counseling (Gielen et al., 2000) with those identified being offered contextualized safety planning (Lindhorst et al., 2005) with referrals to community services such as counseling and legal assistance (Kouyoumdjian et al., 2013).

Given that IPV is said to be responsible for a portion of the loss to followup in the HIV treatment cascade (Mkandawire-Valhmu et al., 2013), incorporating an IPV component into any stage of the care continuum could assist in reducing barriers to care related to IPV. WHO (n.d.) recognizes HCT, in particular, as a critical entry point for IPV detection and response, and suggests that by training 
HIV counselors to assist IPV survivors both HIV and GBV epidemics may be greatly reduced. On the one hand, IPV screening during HCT has been connected with reductions in IPV (Christofides \& Jewkes, 2010), and the literature calls for a scale-up of IPV screening in health care and HIV services (Ahmad et al., 2009; Baig, Ryan \& Rodriguez, 2009; Thackeray, Stelzner, Downs \& Miller, 2007). Yet, on the other hand, because self-protective techniques learned during HCT can "trigger violence" (Christofides \& Jewkes, 2010, pg. 279), supportive services are essential to safe implementation of IPV screening (Matseke, Peltzer \& Habil, 2013). However, research is needed to develop and test interventions that help reduce risks of future violence among those reporting IPV during routine screening in healthcare settings (Jewkes, 2013).

Notwithstanding the charge to create more services jointly targeting IPV and HIV (Abramsky et al., 2012; Matseke et al., 2013; Mkandawire-Valhmu, 2013; WHO, 2001), research on effective programs for this purpose remains scant. A review of the literature found that research is needed to: 1 . Assist with a basic understanding of the HIV-IPV intersection and its social, economic and behavioral dimensions (Pronyk et al., 2006); 2. Develop and test IPV detection methods in resource-limited countries (Baig et al., 2012; Mkandawire-Valhmu et al., 2013); 3. Underscore the breadth of the HIV-IPV phenomenon as well as its causes and consequences among varying populations; 4 . Test the effectiveness of HIV-IPV interventions (WHO, 2001) using rigorous methodology and prioritizing effectiveness in resource-limited countries in sub-Saharan Africa (Jewkes et al., 2006); and 5. Explore strategies for effective safety planning for 
women with HIV who are also IPV victims (Mkandawire-Vahlmu et al., 2013), with a particular need for developing safe partner notification strategies (Kennedy, Haberlen, Amin, Baggaley \& Narasimhan, 2015).

In order for more interventions to effectively target the HIV-IPV intersection, there is a precursory need for the refinement of effective IPV screening techniques aimed at reducing retributional violence. Nonetheless, experts have cautioned that providers must move beyond simply offering IPV screening (Jewkes, 2013), and so while research should test IPV screening protocols for their validity and reliability during the HIV screening process, supportive interventions should be offered in conjunction with screenings. Globally, knowledge is required on the acceptability and feasibility of IPV screenings and interventions (Baig et al., 2009). In particular, there is a call for: 1 . Effective responses to victims identified during screening (Decker et al., 2012); 2. Best practices with implementing and monitoring screenings and results; and 3. Ways to sensitively conduct IPV screenings without causing discomfort or unnecessary difficulties for victims during the process or once they return home (Thackeray et al., 2007).

As stated, much of the extant literature on the HIV-IPV link focuses on IPV as a risk factor for HIV and fails to pay proper attention to IPV among women who are already living with HIV. Interventions, for women with HIV, are equally important, as they can lead to improvements in health and safety. By exploring and circumventing IPV-related barriers to care (Mkandawire-Valhmu et al., 2013), women may more readily access HIV care and experience the long-term benefits 
of suppressed viral loads and reductions in reinfections, effectively contributing to HIV prevention through lowered transmission rates (Jones, Beach, Forehand \& The Family Healthy Project Research Group, 2001). Simply, the HIVIAIDS epidemic in southern Africa will not be halted without a deeper understanding of the experiences of women living with HIV, their continued vulnerability to reinfection and reasons for loss to follow-up along the treatment cascade (Mkandawire-Valhmu et al., 2013); all of which relate directly to the effects of gender inequalities such as IPV.

Finally, there is a dearth of information on how IPV specifically contributes to attrition along the treatment cascade. Despite the great volume of individuals lost to follow-up along the continuum, effective programs for linkage to care, retention and adherence are lacking (Treatment Action group, 2013). Sources urge future research to identify trends associated with delays in linkage to care (Rosen \& Fox, 2011; Reed et al., 2009; Van Zyl et al., 2015) so that programs may be refined to better overcome physical and emotional barriers (Treatment Action Group, 2013; Snyder et al., 2014). This need is greatest among young females (aged $\leq 23$ years) because they have been shown to have high HIV prevalence (UNAIDS, 2012) and the worst linkage rates (Van Zyl et al., 2015).

\subsection{Purpose of Study}

The purpose of this study was to develop and test a risk assessment and safety planning protocol that would mitigate IPV-related barriers to HIV care for South African women testing HIV+ during mobile HCT. To test the intervention, data were compared between the standard of care and two experimental groups 
receiving the dosage levels of 1 . Danger Indicator + an initial safety plan, and 2. Danger Indicator + initial safety plan + follow-up safety plan. The protocol implemented assessed for danger levels relevant to the HIV-IPV intersection and conducted safety planning as a means to mitigate the impact of IPV on HIV outcomes immediately following an HIV+ diagnosis. The protocol was not developed to be an IPV intervention but instead to use psychoeducation on the dynamics of IPV to facilitate safe linkage to care and other HIV-related helpseeking behavior. The concept was to first alter participant's subjective norms on IPV, leading to heightened awareness of IPV in their relationship, and then use the heightened awareness in conjunction with the safety plan to not only catalyze HIV linkage to care but do so in the safest manner. Because this protocol added the new IPV components to a pre-existing evidence-based practice, this study included questions over feasibility and acceptability of the IPV components and was an experimental study exploring the use of two dosage levels.

To date, no interventions have been found that specifically test the efficacy and dosage levels of an IPV risk assessment and safety protocol administered alongside mobile HCT in South Africa, nor that consider the impact of IPV on linkage to care for women recently testing HIV+. No standardized tool could be found for assessing danger associated with the HIV-IPV link, and this study sought to validate such a psychometric instrument. Finally, because no standardized safety planning protocol could be found that addressed the HIV-IPV intersection, the study also aimed to test the acceptability and feasibility of using specific safety-planning strategies. 
The results of this study will inform future research about the effectiveness and required dosage levels of an IPV risk assessment and safety planning protocol during mobile HCT, provide a valid and reliable tool for measuring participant risk and safety related to the HIV-IPV intersection, and provide trends in links to care for those who are HIV+ and experiencing IPV. Successful components of this protocol can be used to assist women who might not have received other formal IPV assistance or who may be at risk of delaying linkage to HIV care due to IPV-related barriers. IPV resources are limited in South Africa, and literature cites that many victims are more likely to show up for health-related services than seek out formal IPV services (Campbell, 2004; Lindhorst et al., 2005). Thus, refining health-related services, like mobile HCT, to include a risk assessment and safety planning protocol could mitigate the effects of IPV and improve health and safety of recently diagnosed individuals.

The study's overall aim was to refine the IPV components, retain participants, avoid contamination, and test effectiveness of the intervention related to participant risk and safety and linkage to care. The main Independent variable for this study was the risk assessment and safety planning intervention, which had two levels. Dependent variables for this study included the primary areas of risk and safety scores and successful linkage to care, and the secondary areas of feasibility, acceptability and satisfaction.

The study was administered in two phases. The aims of Phase I were to: 1). Refine study instrumentation, via focus interviews, to improve cultural sensitivity of instruments as well as feasibility of use (readability, problems with 
wording, etc.), and 2). Refine program components via focus interviews to improve cultural sensitivity of proposed safety plan (phrasing/wording). The aims of Phase II of the study were to: 1). Determine feasibility of the protocol a). by successfully implementing it with $75 \%$ of eligible participants B). and successfully retaining $75 \%$ of participants at one month follow-up; 2 ). Determine acceptability of the protocol A). by receiving a positive rating from at least $80 \%$ of participants, and B). analyzing any significant variations in participant acceptability between the Experimental groups (between dosage levels) and the Standard of Care group. 3). Test the effectiveness of strategies used to avoid contamination of the sample between the Standard of Care and experimental groups with successful avoidance of contamination determined by a low contamination percentage of $\leq 10 \%$. 4). Determine effectiveness of intervention related to A). participant risk and safety scores, and B). successful linkage to care.

\subsection{Research Questions}

\section{Primary Research Questions and Results}

\section{Research Question 1A}

What is the difference in participant risk and safety scores between the combined Experimental groups and the Standard of Care group?

Hypothesis: Participants in the combined Experimental group (receiving any level of the intervention) will have improved scores on the HIV IPV Risk and Safety scale (HIRS) as compared to participants receiving only the standard of care. Research Question 1B 
What is the difference in participant risk and safety scores between dosage levels of those who receive 1 . danger indicator score + initial safety plan, and 2 . danger indicator score + initial safety plan + follow-up safety plan? Analysis was done on pre \& post difference scores.

Hypothesis: Participants with the highest level of dosage will have the highest amount of increased perceived safety as measured by the HIRS.

Research Question 2

What is the difference in participant linkage success between the combined Experimental groups and the Standard of Care group? Successful linkage (within 30 days) and number of days linked were used as indicators.

Hypothesis: Participants in the combined Experimental groups (receiving any level of the intervention) will have increased rates of linkage (more links at a faster rate).

Secondary Research Questions:

Research Question 3A

What is the difference in participant satisfaction scores between the combined Experimental groups and the Standard of Care group?

Hypothesis: Participants in the Experimental group (receiving any level of the intervention) will have higher satisfaction scores as compared to participants receiving only the Standard of Care.

Research Question 3B 
What is the difference in participant satisfaction, feasibility and acceptability scores between dosage levels of those who receive 1. danger indicator + initial safety plan, and 2 . danger indicator + initial safety plan + follow-up safety plan? Hypothesis: The possible impact of higher dosage intervention on satisfaction, acceptability and feasibility is uncertain.

\subsection{Summary \& What to Expect}

The current study was designed to build on the strengths of contemporary evidence-based practices of HCT by incorporating a risk and safety protocol as a method for mitigating IPV immediately following an HIV+ diagnosis. The need for such an approach has been underscored in this chapter, and the results of this study will improve researchers' understanding of women's experience with IPV immediately following a diagnosis and help refine HCT services so that practice initiatives may better address the HIV-IPV intersection. It is urgent that more research focus on mitigating the effects of IPV on HIV outcomes (Shafer et al., 2012), and this protocol is a first attempt at doing so for women in South Africa who have recently tested HIV+ during mobile HCT. Study results will contribute significantly to both the HIV and IPV knowledge base as there is a great need for information on effective safety planning techniques for IPV, in general, and specific to the HIV-IPV intersection, especially among those recently testing $\mathrm{HIV+}$. Findings shed light on the experiences of women in IPV relationships in the first 30 days after testing HIV+ and reveal trends associated with linkage to care for women who currently have IPV in their relationship.

Chapter Two explicates each component of the independent and 
dependent variables. These components are deconstructed and analyzed from a theoretical perspective with empirical support provided for the inclusion of each element. Evidence is provided for best measurement methods for the dependent variables. This explication provides clarity of protocol components, their theoretical foundations, and the mechanisms by which they operate, while also demonstrating the ethics and objectives of the study.

Chapter Three describes the study's methodology. The chapter further explains the study's aims, design, analysis methods, operational definitions of the protocol variables, instruments and program components, support for the inclusion of items, study procedures including participant recruitment, staff training and data collection, and ethical considerations.

Chapter Four provides study results. This chapter describes the sample demographics, methods of data cleaning and incorporates a consort diagram for clarity. It then includes results on the reliability and validity of the study's psychometric instruments, and operationalization of the dependent variables. Finally, the heart of the chapter discusses findings first from Phase I of the study, and then Phase II, beginning with an outline of how the study measured up to its aims, and ending with tables, figures and discussions over primary and secondary research questions as well as additional relevant findings.

Finally, Chapter Five discusses the practical significance of the study's findings and is outlined predominately by research question. Then the study's strengths and limitations are discussed as well as the implications for future practice and research. 


\section{CHAPTER 2}

\section{LITERATURE REVIEW \& THEORETICAL BACKGROUND}

Chapter One provided an introduction of the study's topic, underscored the need for the current study, and briefly introduced the independent and dependent variables. This chapter provides a deeper explication of each variable by presenting empirical support and theoretical underpinnings of each component of the HIRS protocol. Discussion begins with the independent variable, explaining each component of the HIRS protocol, and ends with a review of best practice evaluation measurements for each Dependent variable: linkage to care, safety, feasibility, acceptability and satisfaction.

The HIV IPV Risk \& Safety (HIRS) Protocol

The Independent variable for this study is the HIRS protocol (HIV IPV Risk and Safety), or intervention, which was implemented in two dosage levels. A greater description of this protocol may be found in Chapter Three. However, a brief description of the protocol's three components follows. The first component was the standard of care service, which included mobile HIV Counseling and Testing (HCT) utilizing an evidence-based edutainment 
approach and a Links to Care call centre program. All participants in the study received this portion of the protocol approximately one day after testing HIV+ during mobile HCT. The second component was the risk assessment, as measured by the Danger Indicator. While all participants were issued the Danger Indicator, only the Experimental groups were informed of their danger levels as an intervention component. The final component was a safety plan, which was administered in two dosage levels. Both Experimental groups received the initial safety plan, administered during the initial phone call, immediately following the danger indicator score. Only Dosage Level 2 group received a follow-up safety plan, administered approximately two weeks after the initial phone call and approximately two weeks before the post-test. All individuals received a post-test approximately one month after their HIV testing date.

An important note about the HIRS protocol is that it was not administered as an intimate partner violence (IPV) intervention but, instead, as an HIV intervention that works towards reducing barriers to care associated with the HIVIPV intersection. Therefore, the protocol recruited participants from an HIV testing program and its main focus was on facilitating linkage to HIV care-post HIV diagnosis-in a safe and satisfactory manner. To adequately explain this protocol, the HIV testing approach will first be explained. This will be followed by a theoretical explanation of how IPV impacts help-seeking behavior and the mechanisms by which this protocol attempted to attenuate impairments in help seeking or barriers associated with the HIV-IPV intersection. 


\subsection{Empirical Support for the Standard of Care}

Component One of the Standard of Care: Mobile HCT

Mobile HCT, sometimes referred to as community-based HIV Counseling and Testing, is a service that counsels individuals on HIV testing options, tests results, and HIV prevention techniques; this is seen as the pre-test, post-test and follow-up counseling trifecta. The counseling provides education so that individuals can make informed and voluntary decisions about HIV testing uptake. Services are offered in a safe and confidential space and results are followed by emotional support. Individuals testing HIV+ are evaluated for their risk for HIV transmission and encouraged to link to further HIV care. Because individuals testing HIV+ may go on to experience stigmatization with many negative consequences, VCT endorses only voluntary counseling (UNAIDS, 2000). Although the phrase has been dropped from the newer acronym, HCT also endorses only voluntary testing and is used as synonymous with VCT. In summary, VCT acts as both an HIV preventative measure and entry-point for HIV care (UNAIDS, 2000).

Although HCT has been linked with reduced transmission rates, uptake is

often cited as poor (Mall et al., 2013; UNAIDS, 2000), and innovative approaches are needed to reduce barriers to VCT. One way to reduce barriers is to make services more accessible or population-tailored; some examples are those that are more physically convenient (as with hours of operation and/or location, etc.), or those that help reduce psychological barriers by offering services that challenge stigma (UNAIDS, 2000). 
Because HCT has traditionally been offered in a clinic-based setting (Khumalo-Sakutukwa et al., 2008), mobile HCT is an innovative alternative that enables services to be more physically accessible. Mobile HCT is offered as a roving pop-up service, enabling provision boundlessly, and impervious to physical limitations. Also known for being a safe testing method, mobile HCT services are anonymous, confidential, rapid, and free. Its accessibility has been shown to increase testing uptake, alter perceptions about testing and spread community awareness about HIV (Khumalo-Sakutukwa et al.). Historically, South Africans have branded HIV as a private matter of individual pathology, but the ensuing need for community responsibility and response has been documented (Lippman et al., 2013). Following the 2010 South African government campaign to increase HIV testing in nonmedical and mobile forums (Bassett, Govindasamy, et al., 2014), mobile HCT has increased in popularity and facilitated a needed shift in HIV testing norms (Khumalo-Sakutukwa et al., 2008).

Component Two of the Standard of Care: The Edutainment Approach

Another component of the specific mobile HCT service provided as the standard of care in this study was an empirically supported edutainment approach. This particular edutainment approach combines HCT with technology and health messaging. Potential testers view a celebrity-based HIV prevention video before deciding if they will engage in testing. These videos contained health messaging from hip local South African celebrities on HIV risk reduction methods (safe sex, HIV testing, etc). The video was shown to de-stigmatize HIV testing, and results show it was effective in improving HCT uptake among young 
black participants (with the celebrities also being black) (Van Zyl \& Barney, 2014). Further, this approach was also found to increase HIV knowledge and reduce fears associated with testing (Van Zyl, Barney \& Pahl, 2014).

Component Three of the Standard of Care: Links to Care Call Centre program

The final component to the mobile HCT utilized in the standard of care service in this study was the Links to Care call centre program. This program also has empirical support for its use among South Africans (Van Zyl et al., 2015). While offering HCT through the mobile medium has assisted with testing uptake, most individuals living with HIV remain without needed medical care because they delay linkage to care (Lawn et al, 2006; Losina et al., 2010; Bassett et al., 2010; Kayigamba, Bakker, Fikse, Mugisha, \& Asiimwe, 2012). The Links to Care call centre program utilizes a call centre to make daily contact with individuals who have recently tested HIV+. Mobile phone contact has been shown to be an effective way for healthcare providers to stay connected with youth for test results (Van der Kop et al., 2013) and appointment reminders (Jewkes et al., 2006; Zhang \& Chen 2013), and this program has been used to encourage linkage to care after HIV testing and diagnosis, with an average linkage to care rate of $51 \%$ within 31 days (Van Zyl et al.).

\subsubsection{Theoretical Background of the Standard of Care}

Several theoretical frameworks collectively support the effectiveness of the standard of care service. Applicable models and theories are the Health Belief Model, the Theory of Action/Planned Behavior, Social Ecological Model, 
Social Movement Theory, Diffusion of Innovations Theory, and Entertainment Education Theory.

As the most basic explanation, the health belief model demonstrates that individuals will act to avoid poor health. Individuals must first perceive themselves to be at risk and then discern the possibility for risk high enough that action should be taken to avoid harm. One criticism of this model is that it does not consider the role of social pressure or even external forces in the continuation of risky behaviors despite perceived risk of harm and desire to avoid illness (NAM AIDSmap, 2016). However, the Theory of Planned Behavior suggests that congruency in intentionality, attitude and subjective norms increases one's sense of self-efficacy to achieve a desired behavior and leads to a greater likelihood of action towards such self-protective behavior, despite any dissonant external forces (Ajzen, 2006). Thus, the premise of HCT is to effect changes in attitudes and subjective norms as a means for buttressing intentionality and catalyzing congruent action.

Although these models help explain, generally, why someone would be motivated to engage in HIV testing, it is the combination of Social Ecological model and Social Movement Theory that elucidate how HIV testing in non-clinical settings has become an available and effective approach. Social Ecological model first illustrates that individuals and their environment are inextricably connected; people are influenced by the environment, and the environment is influenced by people (Chimphamba Gombachika et al., 2012). Social Movement Theory provides a framework for how and why communities begin to mobilize 
around an issue (Lippman et al., 2013). For example, community-based HCT appeared when traditional HIV testing services failed to appeal to certain highrisk populations, and community members subsequently championed efforts to increase testing uptake among those groups. Once these services appeared, a norm around HIV testing was shifted. The pop up mobile tents created a milieu where testing was seen as quotidian. Stigma associated with HIV testing was attenuated, increasing the acceptability of services and, ultimately, uptake.

The final theories applicable to the standard of care service offered in this study are Diffusion of Innovations Theory and Entertainment Education Theory. The celebrity video approach utilized during this mobile HCT was guided by both of these theories. Entertainment Education Theory blends education into the entertainment forum to increase palatability of subject matter and improve knowledge on an issue, as well as attitudes and behaviors (Vaughn, Rogers, Singhal, and Swalehe, 2000). This theory has particularly been used in the creation of HIVIAIDS preventative interventions in South Africa and within the international context (Cardey, Garforth, Govender, \& Dyll-Myklebust, 2013). In addition to blending education with entertainment to increase health-messaging appeal, the edutainment approach was guided by a concept within the diffusions of innovations theory, which has been used to construct HIVIAIDS preventative strategies previously. The theory, in general, seeks to explain how innovations are diffused or spread throughout a social system (Rogers, 1997), but the concept of homophily posits, "ideas are most often transferred between individuals who are alike" (Rogers as cited in Van Zyl \& Barney, 2014). Literature 
supports that individuals are more likely to find HIVIAIDS messaging credible when it is delivered by someone of a shared race (Herek et al. as cited in Van Zyl \& Barney, 2014). This demonstrates, in part, how the edutainment approach has been effective in improving testing uptake among individuals who identify as black.

Incorporating an IPV component into HCT

Chapter One described how linkage to care after HCT has remained low, and IPV was identified as a significant barrier to engagement of care at various points of the HIV care continuum. Literature both underscores the need for services that address the HIV-IPV intersection and offers suggestions for effective joint approaches. The World Health Organization (n.d.) identifies HCT is a crucial gateway for IPV detection and support and recommends cross-training HIV counselors to address IPV during HCT services. More, IPV screening is seen as a vital service for those who are newly diagnosed as $\mathrm{HIV}+$ and experiencing IPV (Mkandawire-Vahlmu et al., 2013).

HCT has been associated with the reduction of sexual risk behaviors (Jewkes et al., 2006) as well the reduction of IPV when routine screenings are conducted (Christofides \& Jewkes, 2010). IPV screening, in general, has been found to be a low risk and inexpensive approach with the potential to reduce revictimization when administered by healthcare providers who display a genuine sense of concern and when referrals are made to increase financial and social support (Ahmad et al., 2009). Because HIV diagnosis and partner notification of HIV+ status have been associated with increased risks for IPV (Mkandawire- 
Vahlmu et al., 2013), IPV screenings during HCT could lead to identifying and assisting those vulnerable to the HIV-IPV link.

Generally, the literature calls for a scale-up of IPV screening in health care and HIV services (Thackeray et al., 2007; Ahmad et al., 2009; Baig et al., 2009). Sources in the U.S. state that screening for IPV in healthcare settings has been shown effective and is now endorsed by the National Association of Social Workers, the American Psychological Association, the American Medical Association and several other large governing bodies; yet, routine screening is infrequently offered (Thackeray et al., 2007). Moreover, self-protective behaviors promoted during HCT (condom use, HIV status disclosure, etc.) can "trigger violence" (Christofides \& Jewkes, 2010, pg. 279), and, therefore, efforts addressing the HIV-IPV link should move beyond routine IPV screening (Matseke et al., 2013) and work towards developing and testing more interventions that effectively reduce risks among those screened (Jewkes, 2013).

\subsection{Empirical Support for Experimental Components of HIRS Protocol} Conceptual Definition of Intimate Partner Violence

Several theoretical frameworks guided the conceptual definition of intimate partner violence used within the IPV component of this protocol. While some of these frameworks diverge at times, this study takes an integrative approach to conceptualizing IPV. First, an explanation of the nomenclature employed will provide baseline clarity. The term intimate partner violence has been used rather than domestic violence because this study focuses on violence occurring in 
romantic relationships (spouse, ex-spouse, current or past partner) rather that perpetrated by any person within a person's domestic realm. The term partner is used to reference a romantic partner (boyfriend, girlfriend, spouse, etc.) as an effort to be inclusive of all relationship types (heterosexual, same sex, etc.).

It is also necessary to provide an illustration of how physical, emotional or sexual abuse manifests in South Africa. Commonly, physical violence appears as slapping, pushing, hitting with a fist or stick, threats of physical harm, or stabbing or shooting. Examples of typical emotional abuse are tactics to control or belittle a partner, flaunting other relationships in a shared home, forcing women and their children out of the home, or denial of essential financial support (when it is available). Like the other forms, sexual abuse occurs on a continuum of severity ranging from persuading a partner to have sex, forcing sex without a condom, or perpetrating violent rape (Jewkes et al., 2002).

The two main theories that have generally been used to explain the dynamics of intimate partner violence, not specific to the South African context, are Feminist Theory and Family Violence Theory (Kurz as cited in Carlson \& Jones, 2010). Feminist theory conceptualizes that IPV is "rooted in patriarchal traditions of male dominance in heterosexual relationships" (Johnson \& Leone, 2005, pg. 323). From this perspective, violence is seen as escalating and largely maintained through tactics of power and control perpetrated systematically by men against women (Home of the Duluth Model, 2011). Evidence supporting this approach largely grew from phenomenological studies of women attending to medical facilities, shelters or legal services for domestic violence (Carlson \& 
Jones, 2010). Family Violence Theory, appearing around the same time as Feminist Theory, posits that IPV is rooted in family conflict, which inherently occurs in all social groups, and results from "the methods by which conflicts are resolved" (Straus as cited in Carlson \& Jones, 2010, pg. 250). Whereas both perspectives hold that IPV is a form of gender-based violence, Feminist Theory places sexism "at the center of the analysis," and Family violence theory views sexism "as only one causal structural factor among many" (Lawson, 2012, pg. 583). The other operative difference in these approaches is that Feminist Theory views IPV as a manifestation of systematic power and control, and Family Violence Theory views IPV as the product of actions taken to address family conflict.

Johnson (2008) argues that framing all IPV as the result of power and control is myopic because the phenomenon is more complex and, conversely, contains varying IPV typologies. According to this theory, the major IPV typologies that still explain male violence against women are Situational Couple Violence and Intimate Terrorism (Johnson \& Leone, 2005). Intimate Terrorism is the typology ordinarily illustrated in the literature on domestic and intimate partner violence, derived from samples of abused women. This form of IPV is rooted in power and control and its victims experience more severe, frequent and persistent violence (Johnson \& Leone). Situational Couple Violence, on the other hand, is more often a typology of gender parity where violence erupts as male or female partners attempt to resolve conflict. In this typology, there may be some efforts to control another person, but there is no general or pervasive pattern of 
control as an exertion of power. While not seen as a typically escalating or systematic form of violence, Situational Couple Violence can be just as violent or fatal. Most of the IPV found in general survey populations is that of Situational Couple Violence (Johnson \& Leone).

The HIRS protocol is based on an integrative approach, including Feminist Theory, Family Violence Theory and some integrative theories that help expand the explanation of IPV such as that of Anderson (1997) and Heise (1998) (Lawson, 2012). Anderson argues that gender and ecology are relevant to understanding IPV, and Heise discusses the need to explore multiple causal factors of IPV. Heise posits that feminist theory "fails to explain why some men beat and rape women when others do not" although all men are exposed to patriarchy (Heise, 1998, pg. 263).

Heise, instead, offers a more holistic and somewhat universal explanation positing that IPV results from many intermingling ecological factors. Her theory describes IPV on a broader level and helps allow the conceptualized causation of IPV to move beyond the limits of individual pathology pitted against socio-cultural power play (1998). Through her theory, IPV is seen as the result of a matrix of causal factors rising from the confluence of individual, microsystem (family), exosystem (institutions and social structures), and macrosystem (cultural norms and attitudes) factors.

To some extent, this theory helps explain the higher occurrence of IPV against women with HIV-i.e. when the culture of gender-based violence in South Africa (microsystem and exosystem) and a stigma-laden HIV status 
(macrosystem) are coupled with individual and family factors such as pre-existing IPV or childhood abuse (Jewkes et al., 2002). According to Heise's theory, these factors intermingle in a concentric circle, sharing the same axis so that one factor feeds into another factor. However, Jewkes et al. (2002) assert that Heise's theory, while involving some international consideration, is reflective of North American ideology; the theory is beneficial and relevant to the South African context but it is also incomplete. Instead, the etiology and consequences of gender-based violence in South Africa are more complex than what can be explained by the ecological framework she proposes.

Although the etiology of IPV has been connected to larger South African cultural norms such as "conservative ideas about the position of women" (Sugarman \& Frankel as cited in Jewkes et al., 2002, pg. 1613), mediators of IPV also shed light on country-specific underlying causes. For example, two different studies found that having some post-school education is protective against lifetime occurrence of IPV (Straus, Gelles, \& Steinmetz as cited in Jewkes et al., 2002). Jewkes et al. hypothesize that the mediator is actually women's social empowerment experienced as a result of their higher education, as augmented social empowerment enables a woman to utilize available resources and consequently increase her agency while decreasing isolation (pg. 1612). Further, the major problem with strictly using the ecological framework to explain IPV in South Africa is that factors such as poverty can be characterized as a larger cultural or societal issue or as an individual factor (as the example above illustrated). While a solid framework for understanding IPV etiology in South 
Africa is still evolving, two fixed factors are "the unequal position of women and the acceptance of certain forms of interpersonal violence" (pg. 1614).

Component One of the HIRS Protocol: The Danger Indicator

Understanding IPV within its appropriate context is integral to the creation or use of a valid IPV risk assessment tool (Thackeray et al., 2007). First, a conceptual definition of IPV risk assessment tools provides clarity for the larger explanation of the HIRS risk assessment tool creation.

Generally, IPV risk assessment tools function exactly as the name suggests. These tools rely on reports of past assault to estimate the amount of relative risk each person has for IPV-related re-assault or lethality by a domestic or intimate partner. Assessments are conducted as a collaborative dialogic process with a victim, perpetrator or both. The point of the assessment is not merely to ascertain knowledge on the likelihood of re-assault but to explicitly use the information procured to assist in the creation of a personalized safety plan that addresses each person's specific risks. Essentially, the risk assessment is the means to the safety plan's end, and an effective safety plan hinges on an effective and thorough risk assessment (Ending Violence Association of BC, 2013).

IPV risk assessments fall into several general categories: clinical, actuarial, structured and unstructured clinical professional judgment approaches (Bowen, 2011; Department of Justice, 2015) and victim's risk-assessing scales (Department of Justice). The clinical risk assessment is utilized most commonly and relies on practitioners' clinical judgment of a person's risk. This type of tool 
has been criticized for poor validity and reliability and its propensity for practitioner bias (Bowen). The actuarial risk assessment uses statistics to predict violent behavior as occurring within a certain amount of time. While some sources claim this approach to have stronger reliability, others disagree and, ultimately, more research is needed to verify each approach's robustness in varying contexts (Bowen). Less research has been conducted on victim risk assessing scales, and, therefore, less is known about the validity and reliability of these scales (Department of Justice).

IPV risk assessments require an iterative process to gauge on-going risks. While current literature does not provide a consistent operationalization for a general IPV risk definition (Kropp as cited in Bowen, 2011), it is important to note that IPV risk factors should be considered within the context for which they are being used for violence prediction (Bowen). Risk assessment is strengthened by not merely considering past or possible future acts of violence, but by, instead, examining the contexts of the violence (frequency, severity and type) and situations that may impact its occurrence (Douglas \& Kropp as cited in Bowen).

One of the more empirically supported risk assessment tools is the Danger Assessment (DA). The DA is an actuarial tool "as it draws upon a retrospective empirical evidence base concerning the risk factors for either intimate partner homicide or severe IPV" (Bowen, 2011, pg. 221). It has been shown to reliably measure potential IPV lethality (Campbell et al., n.d.) and was created to help women appraise personalized risk levels so that practitioners could better assist them in developing a plan for safety (Glass, Eden, Bloom \& 
Perrin, 2010). Most other IPV risk assessment tools measure risk for re-assault rather than femicide and normally also rely on collateral information-gathering (police records, perpetrators, etc.) rather than just reports from a victim (Messing \& Thaller, 2013). Although sources conflict about which IPV risk assessment approach is best, Campbell (2004), a creator of the DA, explains that many women have the most accurate assessment of their own risk for fatality, with one study showing that approximately half of the women were accurate (Campbell, 2004). Heckert \& Gondolf (2004) found that using the combination of obtained risk markers, based on a woman's perception, in conjunction with the Danger Assessment was the best prediction of re-assault.

Component Two of the HIRS protocol: Safety Planning

Once the risk assessment information has been procured, it is then used to create a person-specific safety plan. Safety planning is considered the benchmark intervention for addressing IPV (Campbell, Webster \& Glass, 2009; Bloom et al., 2014; Glass et al., 2010) and is suggested as a tool to be used following IPV screenings (Thackeray et al., 2007). The information gathered during risk assessment is contextualized, with each person's particular circumstances, and synthesized into a plan to help mitigate future harm (Thackeray et al., 2007). This plan considers personal interpretation of the violence (Dienemann, Campbell, Landenberger \& Curry, 2002), levels of danger, resources, priorities and goals (Davies, Lyon, \& Monti-Catania as cited in Bloom et al., 2014, pg. 243; Mkandawire-Vahlmu et al., 2013).

Although safety planning is critical to improving a person's safety, most 
victims do not seek formal services for IPV (Lindhorst et al., 2005) and never receive such assistance. Some individuals are not aware of the existence of formal IPV services or how to access them. Others may be distrusting of providers, fear the loss of privacy, be too fearful or ashamed, or even worry about shaming their partners (Bloom et al., 2014). Considering that IPV is occurring at epidemic rates throughout the world (WHO, n.d.) and victims are more likely to show up for other types of social services, more IPV risk assessment and safety-planning protocols should be conducted "in all contexts by generalist practitioners" (Lindhorst et al., 2005, pg. 331).

Sources repeatedly laud the effectiveness and necessity of safety planning; yet, there is a dearth of information on effective strategies, and the literature that does exist is still in its nascent stages. Parker \& Gielen (2014) highlight that "little is known about the duration of use of safety strategies, the strategies actually discussed with women during safety planning, or whether women use multiple strategies at the same time..." (pg. 591). Therefore, while we know that safety planning, in general, is effective, and we know some specific strategies are shown to reduce and others increase revictimization, more research is needed to define reliable components of safety planning and explore risks and benefits of specific safety strategies (Parker \& Gielen, 2014).

Based on the systematic review of literature on IPV safety techniques, Parker \& Gielen (2014) assert that IPV safety planning generally falls into 6 domains. Each domain includes a strategy victims report using. The first three domains cover victim engagement with support systems and resources. The final 
three domains cover strategies victims either use or avoid for optimal safety. Domain one considers victim engagement with formal networks such as medical services, clergy, counselors and IPV-specific services. Domain two considers victim engagement with informal networks such as friends and family. Domain three considers victim engagement with legal services. Domain four involves the use of placating strategies such as "trying to avoid the abuser at certain times, doing whatever the abuser wants, and trying to keep things quiet from the abuser" (pg. 590). Domain five involves the use of resistance strategies such as "fighting back physically, putting a weapon where she could get it to protect herself, trying to end the relationship, and running and hiding" (pg. 590). Finally, Domain six involves the use of general safety planning techniques like "trying to avoid the abuser at certain times, doing whatever the abuser wants, and trying to keep things quiet from the abuser" [...] "hiding money or keeping important phone numbers to use" (pg. 590).

These enumerated strategies highlight safety planning techniques ground in standard IPV practice, but research is needed to show safety planning's efficacy in the context of HIV. Strategies should always be contextualized to each woman's self-perceived threats, options and abilities as a means to help her identify personalized risks and protective factors in her situation (Lindhorst et al., 2005). In addition to a victim's perception being key to the appropriation of each of these strategies, the safety plan would also need to address the nuances of the HIV-IPV intersection.

Areas relevant to safety planning, with those living with both HIV and IPV, 
are outlined by the National Network to End Domestic Violence (NNEDV) (2014). HIV-IPV specific safety planning addresses many of the standard areas found in the IPV power and control wheel, with the additional area of Medical Abuse. However, each spoke of the power and control wheel, representing tactics used by abusers, is formulated in the HIVIAIDS Power and Control Wheel to reflect how abusers specifically harm individuals living with both HIV and IPV (figure 2.1). Although safety planning strategies specific to the HIV-IPV intersection have yet to be adequately tested, an effective HIV-IPV safety plan would logically work to mitigate the tactics found within the HIV-IPV Power and Control Wheel and address any other forms of abuse found to be commonly used against those living with HIV (CDC, 2014). Some of these areas include educating victims on healthy relationships, altering norms around abuse (CDC), creating plans for safely getting to medical appointments, taking medication (NNEDV, 2014), and safe partner notification (Ramachandran et al., 2010).

Take partner notification (or disclosure) of serostatus as an example: UNAIDS (2000) stresses that although it is important to disclose one's serostatus to one's partner to initiate practices of safer sex, this may not be possible for women experiencing IPV "who face abuse or abandonment if known to be seropositive" (pg. 5). Consequently, strategies to facilitate non-violence upon partner notification are vitally needed for women affected by both HIV and IPV (Kennedy et al., 2015; Maman, Mbwambo, Hogan, Kilonzo \& Sweat, 2001). In the process of planning safe disclosure strategies, individuals should be empowered to make their own decision as to whether or not they will disclose to 
their partners, given that each situation is unique and each person must determine if it is safe for her to disclose (A le Roux-Kemp, 2013).

Figure 2.1 HIVIAIDS Power and Control Wheel

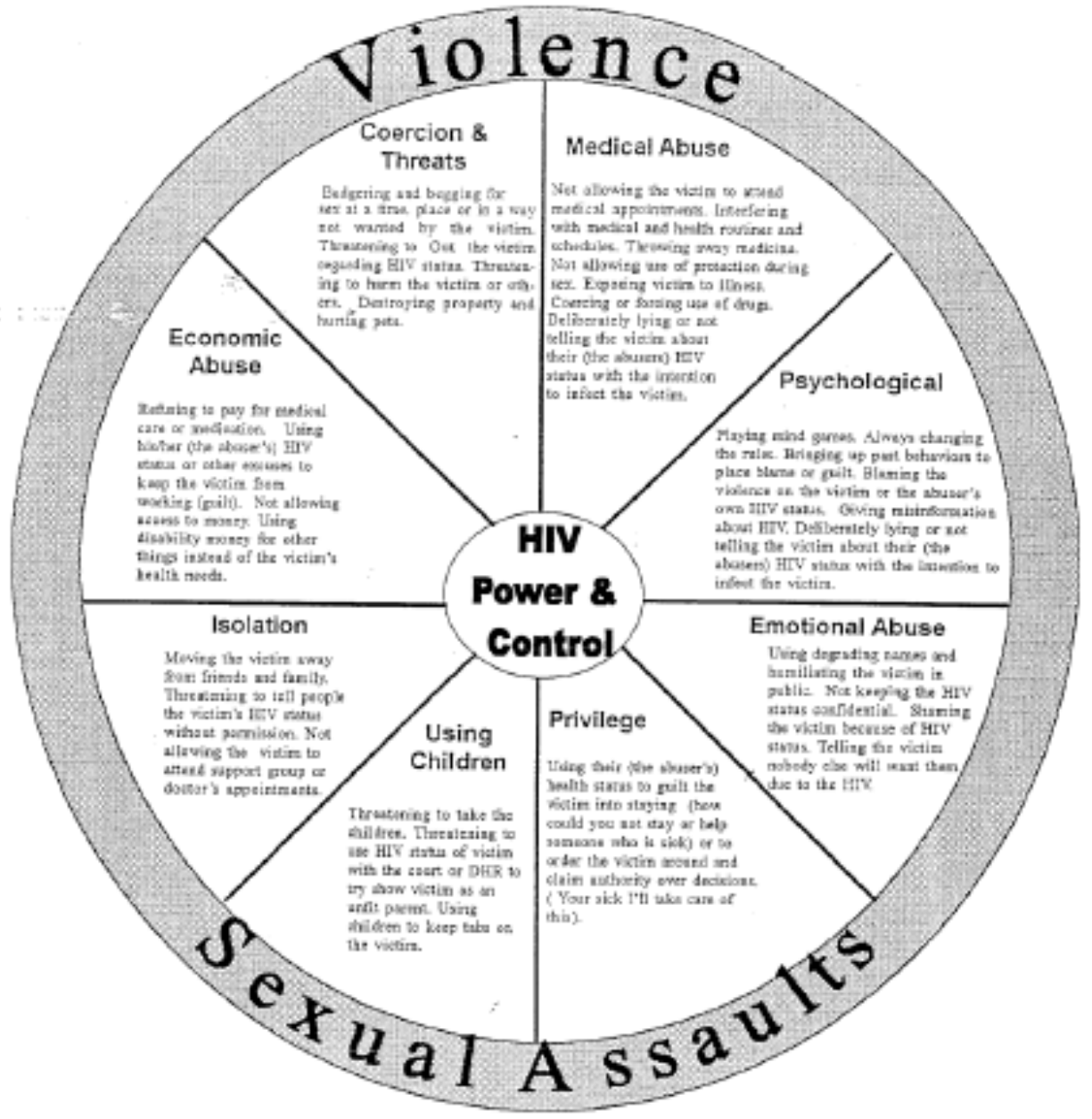

Figure 2.1 HIVIAIDS Power and Control Wheel. National Network to End Domestic Violence. (2014). Positively Safe: The intersection of domestic violence and HIVIAIDS. HIVIAIDS Power and Control Wheel. Alabama Coalition Against Domestic Violence \& Motgomery AIDS Outreach. Based on the Power and Control Wheel developed by the Domestic Abuse Intervention Project, Duluth, MN. () July 2014.

Finally, because thorough IPV risk assessment is said to rely on an iterative process, safety planning may also require more than one contact for thoroughness. The Ending Violence Association of BC (2013) describes risk 
assessment and safety planning as dynamic processes so that when conducting them providers must expect changes in victim's circumstances and the possibility for needed revisions. Hence, revising safety plans over time can be beneficial for survivors to anticipate changing needs.

\subsubsection{Theoretical Background of the HIRS Protocol}

The theoretical models relevant to the HIRS protocol are Crisis Theory, Stress and Coping Theory, Lindenberger's Theory of Entrapment and Recovery, the Transtheoretical model, Empowerment Theory, and Theory of Action/Planned Behavior.

Crisis Theory was used to guide the stages and some content of the study's intervention. Crisis Theory may be applied both as a conceptual theory, explaining the crisis phenomenon, and as an intervention theory, directing interventions to effectively mitigate crises. Conceptually, Crisis Theory emerged in the 1940's from the work of theorists Erich Lindemann and Gerald Caplan and was used to explain and predict responses to traumatic events. A crisis is defined "as the perception or experience of an event (genuine harm, the threat of harm, or a challenge) as an intolerable difficulty" for which a person's typical coping mechanisms cannot sufficiently manage the event (Walsh, 2013, pg. 306). The formal modality of crisis intervention was created in the 1960's and focuses on attempts to help an individual either return to pre-crisis functioning levels or to grow despite catastrophe. 
Some major concepts of Crisis Theory are stress and crisis. Stress is defined basically as demand exceeding resource. Crisis occurs in three stages: first the person abruptly experiences a rise in tension. Next, the person attempts to manage the stress but fails to adequately do so; the person then becomes overwhelmed by the inability to cope and is more open to receiving assistance. Finally, the person returns to pre-crisis levels (Walsh, 2013, pg. 309-310). The return to pre-crisis levels regularly occurs about four weeks later and the person experiences resolution either through adaptive or maladaptive coping mechanisms (Walsh).

The main concepts of crisis stages, stress, and coping help inform the final relevant areas of crisis theory: assessment and intervention. Coping, as referenced above, is a person's attempt to manage the difficulties of the stressor and a person may cope biologically, psychologically or through the help of social supports (Folkman as cited in Walsh, 2013). Crisis intervention requires an ordered response to individuals in crisis, beginning with assessment and ending with planning and referral. According to the theory, all people should: 1 . Experience a supportive worker/client relationship; 2 . Be encouraged to express their feelings about the challenge at hand; 3 . Receive an assessment to identify precipitating factors and resources such as internal and external coping mechanism—capacity to function adaptively and available social supports, respectively; 4. Be given time for processing the meaning of the assessment; 5 . Help develop an intervention plan based on meaning making of the assessment; 6. Receive "referral and linkage" (pg. 314) with resources; and 7. Receive a 
formal termination or follow-up discussion as a time to meditate on means for averting future crises (Walsh, 2013).

Situational crisis is most germane to the intersection of HIV and IPV as it is defined as "uncommon, extraordinary events that a person has no way of forecasting or controlling" (Walsh, pg. 310). An individual already experiencing IPV and receiving an HIV diagnosis is likely to be experiencing multiple crises, but most acutely the HIV diagnosis will likely spur a new crisis at some point following a diagnosis. Each person experiences a unique response to the diagnosis, but those already experiencing IPV encounter increased difficulties due to the dynamics of the relationship (Davis, 2012). It cannot be assumed that an individual will experience a sharp crisis immediately following an HIV diagnosis, but it is a working assumption that a crisis ensues at some point following the diagnosis and is related to the intersection of it (HIV) and IPV (see Chapter One). The HIRS protocol follows the 7 steps outlined for crisis intervention and does all of which within the timeframe of four weeks. Greater detail is provided in Chapter Three, but the steps most applicable to this theoretical discussion are assessment and planning. Risk assessment is the first action in the sequence from which safety planning is crafted.

The risk assessment component of this study is also informed by Stress and Coping Theory, which is rooted in Stress Theory. Krohne (2002) explains that theories of stress consider either the physiological or psychological response to "external demands (stressors) and bodily processes (stress)" (pg. 2). Fundamental concepts of Stress Theory are appraisal and coping. Appraisal is a 
person's assessment of how an event impacts their welfare, and coping is the person's cognitive and behavioral responses as they attempt to withstand that impact (Lazarus as cited in Krohne, 2002, pg. 3). The Lazarus Theory considers the psychological responses to these stressors, in which stress is viewed as a "transaction' between individuals and their environment" (Krohne, 2002, pg. 3).

From this, Stress and Coping Theory grew and is described as focusing on a person's assessment, or meaning making, of a situation and their evaluation of the best way to cope with the situation based on their available resources (Lindhorst et al. 2005). Stress Theories inform the IPV risk assessment process so that it includes assessing each person's unique risks for danger and potential protective factors (Lindhorst et al., 2005) as a means to inform the most appropriate safety plan in response to the risks identified.

Theories pertinent to enacting a safety plan are Lindenberger's Theory of Entrapment and Recovery, the Theory of Action/Planned Behavior, Transtheoretical model and the Empowerment model. Lindenberger's theory posits that a woman's decision-making in an abusive relationship is influenced by the "social context and the need to balance care for others and herself" (Dienemann et al., 2002, pg. 221). Through this theory, it can be said that a woman will only "decide" she is ready to end an abusive relationship, or take action that could decrease the abuse, once she begins to believe she is not to blame for the abuse (Landenberger, 1989). Employing this theory alone could dangerously begin to shape the hypothesis that a woman is responsible for preventing the abuse or that it is in her control to end the relationship. However, 
the enriching component of this theory is that it emphasizes the need to help a victim break the cycle of power and control that has entrapped her in the belief that that she is responsible. Hence, this theory explains how educating a woman about victim-blaming as a component of abuse may be instrumental in her process of recovery from the abuse, which according to the theory is said to be a non-linear process of moving on.

Receiving psychoeducation on the dynamics of IPV assists a survivor not only with the ability to recognize the presence of abuse but also may provide a shift in the cultural and subjective norms associated with abuse. As stated before under the Conceptual Definition of IPV section, in the South African context, abuse is perpetuated not only by gender inequalities but also by the acceptance of gender-based violence as a normative. Therefore, the Theory of Action/Planned Behavior is again applicable to this component of the protocol in that the idea is to impact changes in a survivor's subjective norms as a means to galvanize change or increase change-directed behavior that is congruent with a new subjective norm. Considering this protocol does not attempt to be an IPV intervention but an HIV intervention that works towards reducing barriers to care associated with the HIV-IPV intersection, it is paramount to underscore that change is used in this discussion to indicate action towards health-related help seeking behavior or, specifically, linkage to HIV care. Change here does not indicate an end goal of women leaving abusive partners or taking action towards breaking subservient roles with partners. Instead, the process of change focuses 
on influencing women to take action towards healthiness (theory of action/planned behavior) despite the influences of external forces.

There is evidence that women in abusive relationships who are considering making a change go through a similar readiness to change process as seen with other change processes. According to the Transtheoretical Model (TTM), one must be ready, willing and able to make a change (Miller \& Rollnick, 2002). Although Motivational Interviewing was not used as an intervention nor was TTM as a predominate approach in this protocol, the work of Miller \& Rollnick explains how change occurs on a theoretical level. More, TTM has been used particularly in sub-Saharan Africa to understand stages of readiness to change among women in IPV relationships (Wagman et al., 2013).

An exploratory study of TTM with abused women showed that those in early stages of change engage in the cognitive processes of consciousnessraising, self-reevaluation, dramatic relief, environmental reevaluation and social liberation (Burke, Denison, Gielen, MacDonnell, \& O'Campo, 2004). Women reported that the psychoeducation they received on abuse assisted them in moving from precontemplation to contemplation by way of consciousness-raising. Through these processes, women reported being able to remain in the relationship while more effectively avoiding violence. Burke et al. explain that while Prochaska's theory of stimulus control has typically been used to explain how individuals can change their own behavior, the tactic of stimulus control was used among the sample as a means for reducing triggers for violence when women altered their own responses during precipitating events. 
If the Theory of Action/Planned Behavior explains the shift in motivation or readiness for change (altered subjective norms increases willingness to change), then the final impetus for change may hinge on actual or perceived ability to change. IPV interventions using TTM work towards affecting large, communitylevel change as a means for supporting a community member's ability to change (changes in community subjective norms increase person's ability to change). Because this is not an IPV intervention, change was not attempted on a community level but builds on the work of an HIV intervention that has already targeted change at the community level. Smaller scale changes were sought for the IPV component of the study as a means for catalyzing health-related help seeking behaviors. Thus, the protocol attempted to impact shifts in perceived ability to change.

When a person perceives they possess the ability to take action towards goal attainment, it is referred to as self-efficacy, and this is a key concept in the mechanism by which methods of empowerment work. Individuals are empowered to take action when they are supplied "with requisite knowledge, skills, and resilient self-beliefs of efficacy to alter aspects of their lives over which they can exercise some control" (Ozer \& Bandura, 1990, pg. 472). Ozer \& Bandura avow that the use of self-empowerment as a means to protect one's self from sexual and physical violence is important given that $80 \%$ of sexual assailants attack without the use of weapons but through other forms of physical force, coercion or intimidation. In sum, the role of self-efficacy could be instrumental in a woman's ability to protect herself when violence arises. This 
source points out the important and implicit caveat that women are not solely responsible for the outcome of these transgressions. Instead, such acts are seen as societal problems that should be tackled by advances in issues related to gender inequalities. In their study investigating the role of perceived coping and self-efficacy in response to physical violence, two mediators were shown to jointly impact behavior, a person's perception of their vulnerability for risk and their sense of self-efficacy. This suggests that perceptions of risk coupled with a belief in one's ability to protect one's self may increase protective behaviors, and ultimately, safety.

Two components that enable someone to implement a safety plan are sense of empowerment (sometimes referred to as agency) and the deployment of social supports. By empowering individuals, providers help each person uncover the resource available to them (internal and external) with the objective of raising "the person's "awareness of oppressive tensions and conflicts in their lives, [and] to help them find ways to be free of these constraints" (Pinderhughes as cited in Dass-Brailsford, 2007, pg. 72). Services using an empowerment model typically use a collaborative approach to focus on improving the well-being of those who have been oppressed or marginalized, and hold the assumptions that people are key players in their own change process, have inherent strengths and capabilities and are interconnected with their environment (Dass-Brailsford, 2007). When applying the empowerment model to a crisis intervention, assumptions are that crises are different for everyone and responses should be tailored to meet each person's needs. Because people are the experts on their 
own lives, services should focus on uncovering each person's individual strengths and connecting them to resources (Walsh, 2013).

Applying the empowerment model to the etiology of IPV, abuse is seen as something that occurs because of an abusive person's propensity to exert power and control over their partner and not because of any personal attribute (class, race, etc.) of the victim (Payne \& Wermeling, 2009). IPV services operating from an empowerment model hold that all victims reserve the right to have access to information, education, and other necessary social and economic support to make informed decisions that best reflect their interests and needs. Rather than attempting to eliminate the violence, which is not controlled by victims, the empowerment approach uses knowledge dissemination, training, and counseling to create a set of services that victims control, such as post-victimization assistance and risk minimization (Ofstehage, Gandhi, Sholk, Radday \& Stanzler, 2011, pg. 3). An example of how these theories culminate to explain the intention behind the protocol would be (see Figure 2.2): A person initiates HIV testing (reasons discussed under theoretical underpinnings of HCT component of protocol), learns of their diagnosis, an acute crisis ensues (at some point typically) and they begin an attempt to cope with the crisis. Those with IPV would theoretically have an impaired ability to employ adaptive coping methods and would be in a state where their acceptance of help would be heightened. Although at this stage they may not be aware of the role of IPV in their relationship, through the risk assessment and safety plan they are informed of 
the danger levels present in their relationship and asked to reflect on this (meaning making). From here, the safety plan is developed and largely involves the deployment of social supports as the primary coping strategy as aligned with crisis intervention. The follow-up safety plan is offered as a means to test if additional psychoeducation on IPV is a necessary reinforcement to stimulate health-related help-seeking behaviors (take action according to the Theory of Action/Planned Behavior).

Figure 2.2 Contextualized Assessment for Strategic Safety Planning with Battered Women

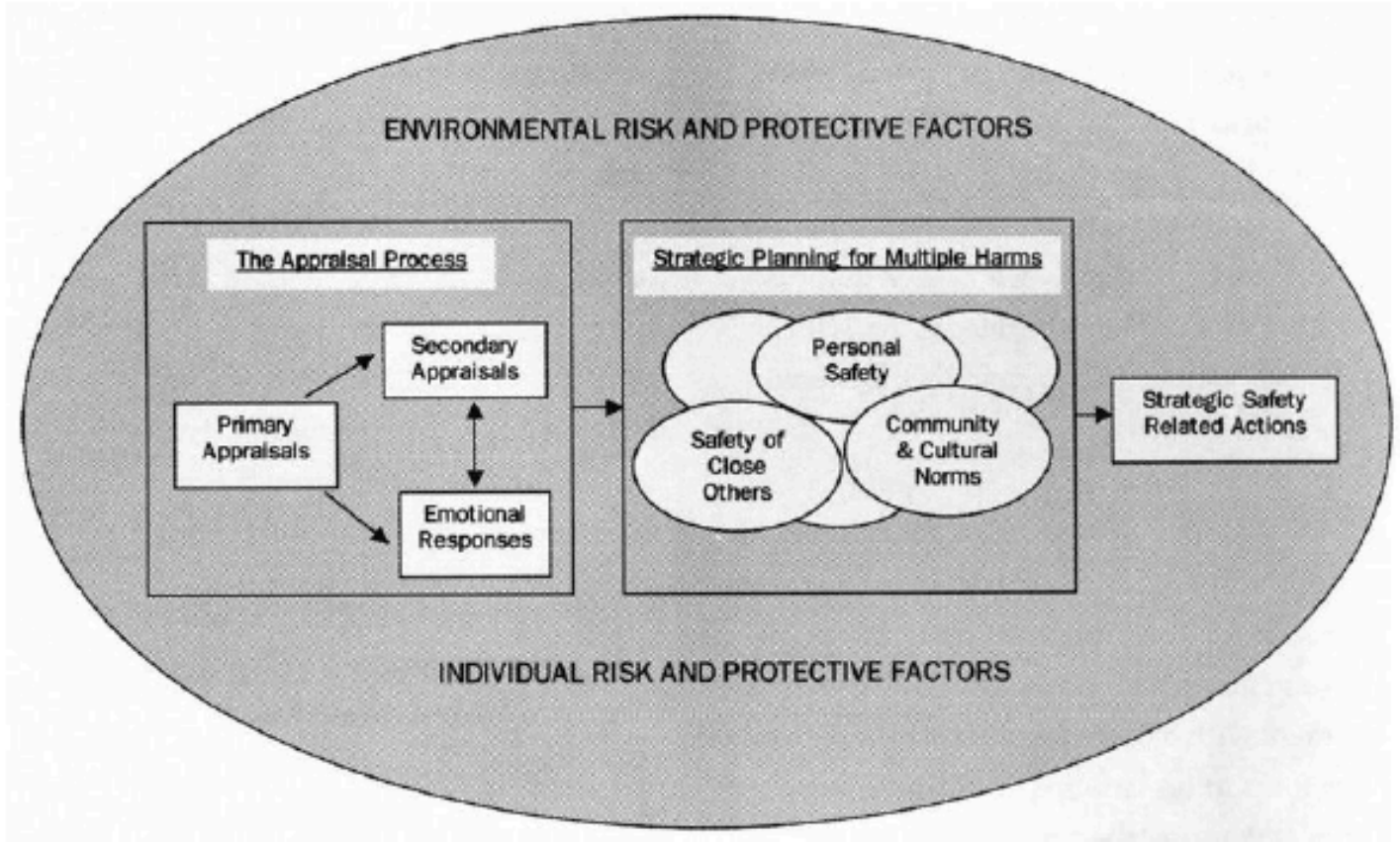

Figure 2.2 Contextualized Assessment for Strategic Safety Planning.... with Battered Women. Lindhorst, T., Nurius, P., Macy, R.J. (2005). Contextualized assessment with battered women: Strategic safety plan to cope with multiple harms, J Social Work Education, 41(2), 334.

\subsection{Evaluating the Intervention \& the Implementation Process}


The development of an ethical and effective intervention relies on the foundation of strong theoretical and empirical support. Chapter One and the beginning of Chapter Two laid out the foundation for the development of the intervention as well as theoretical explanations to describe the processes by which the intervention operated. This latter half of Chapter Two concentrates on describing the dependent variables of linkage to care, perceived safety, and the evaluation components of feasibility, acceptability and satisfaction used for measuring the impact of the protocol. The rationale for the inclusion of each variable will be provided as well as best practices for measuring the component.

The development and implementation of the intervention was informed by the principles of implementation science, as the intervention was built from an inter-disciplinary integration of several theories and sought to understand and design an effective means for implementing the intervention (May, 2013, pg.1). Yet, implementation theory, focusing on uncovering relevant components of the implementation process as well as outcomes (May, 2013), influenced which outcomes were included as dependent variables.

The main aims of implementation theory are to 1 . Describe the protocol process so that research and practice are clearly synthesized, 2. Use theory to explain how outcomes are influenced, and 3. Evaluate the intervention or the actual process of implementation (Nilsen, 2015). The discussion on the dependent variables will touch on all of these aims but will largely focus on providing a rationale for chosen outcome and evaluation variables. Literature in the respective areas of HIV and IPV were exhausted to uncover the standard 
benchmarks used in each discipline. These best practice measurement sticks were integrated into the protocol, as a means for procuring data that would most saliently compare findings with extant knowledge.

\section{Measuring Linkage to Care}

Definitions of the term linkage to care vary widely among current literature. Universally, sources use this term to mean that a person with HIV has linked to some type of medical care, but variations exist in both components of the phrase-linkage (when and where) and care (the nature of what is being received). Furthermore, delays in linkage are defined differently depending on study follow-up periods. This section will describe how other sources have defined this term, and comparisons of findings will be presented in Chapter Five.

Definitions of delays in care range from more conservative estimations of linkage as $>30$ days to broader timeframes such as $>6$ months. For example, whereas Elul et al. (2014) used the marker of 30 days as the endpoint for linkage to care, Losina et al. (2010) defined a loss to follow-up as not linking to care within 8 weeks of a diagnosis. Bassett, Regan et al. (2014) defined linkage to care as either retrieving CD4 cell results or completing an ART literacy training within 90 days from an HIV diagnosis, and Reed et al. (2009) defined delays in linkage for those after 3 months. Another source followed participants for 6 months but this also included observation of ART initiation and death (MacPherson et al. 2012). An alternative but common practice is using CD4 cell stratum to guide and gauge linkage rates so that those with lower CD4 cell counts are advised to link to care sooner given the urgency of their need for care 
(Krazner et al., 2012; Govindassamy et al., 2013). Linkage to care may refer to going to a clinic for CD4 cell results (Losina et al. 2010) or may indicate linking to further medical care after CD4 cell results have been received. The latter is more often the case in studies using point of care CD4 testing, in which an individual receives both an HIV+ diagnosis and CD4 cell results in the same visit (Wynberg, Cooke, Shroufi, Reid \& Ford, 2014).

The importance of timely linkage to care has been cited by many sources. Delayed entry to care has been connected to lower CD4 cell counts (Mugglin et al., 2012; Patten et al., 2013; Van Zyl et al., 2015) and premature death Kayigamba et al., 2012; Larson et al., 2010; Van Zyl et al.). However, linkage to care earlier in the disease cycle has been shown to improve health outcomes (Bassett, Govindasamy et al., 2014) with the timeliness of linkage to care being critical to patients reaping the maximum benefits from ART (Losina et al. 2010) and an important aspect of reducing secondary infections (Jenness et al., 2012). Regrettably, a large number of individuals delay entry to care and initiate ART "with far too advanced immunodeficiency" (Lawn et al., 2006, pg. 770). Therefore, improving linkage to care is essential to actualizing the potential health benefits of ART (Mayer, 2011), but research on the pre-ART period (post diagnosis) remains scant (Mugglin et al., 2012).

Measuring safety

Stopping violence, by way of ending an abusive relationship, has been the touchstone evaluation for many initiatives addressing IPV, but this type of benchmark is problematic because it 1 . Perpetuates victim-blaming by assuming 
victims are responsible for perpetrators' behavior (Dienemman et al., 2002), 2. Assumes abuse will end if the relationship ends, and 3. Presumes women want to leave their partners (Dienemman et al., 2002).

Arguments against victims being responsible for perpetrators' behavior have already been presented above in the theoretical explanation of the mechanics of IPV. The assumption that abuse will stop if someone leaves his/her partner is counter to the perilous truth that the most dangerous time in a violent relationship is when a victim attempts to leave (Halket, Gormley, Mello, Rosenthal, \& Mirkin, 2014). In actuality, more than half (70\%) of all violence happens after a woman has left her partner (Halket et al., 2014), and the generic prescription of an exit plan for the relationship could lead to re-assault (Lindhorst et al., 2005). Contrary to the aforementioned false assumptions, many women do not want to end their relationship and those who do commonly leave many times before they leave for good, with each time being more dangerous than the last (Heckert \& Gondolf, 2004).

In light of these dynamics, in can be presumed that measures of safety often are not rendered from black and white assessments with equifinality but rather are more complex processes. Because safety plans should be informed by each woman's personalized goals, extracted from her subjective interpretation of the abuse (Dienemann et al., 2002, pg. 221), each person's end goal for safety will be different. Safety, instead, may be viewed as a subjective construct with multifarious endpoints. 
Yet, for the purpose of quantifying this intricate concept, best practices in IPV service were reviewed and two thematic approaches were discovered for gauging changes in safety before and after receipt of services: victims' reports of using the strategies (coupled with finding them helpful) and re-assault experienced. A third potential category would be changes in perception of danger, but victims' perception of danger may not be an accurate indicator of safety because it can fluctuate with the person's mental health at the time of the assessment. For example, abuse survivors with posttraumatic stress disorder may have a heightened response to questions triggering an arousal of traumatic memories and responses may be influenced by intrusive thoughts of past events (Cattaneo, Bell, Goodman \& Dutton, 2007). Further, those with worse IPV experiences or those considering making a relationship change may report a heightened sense of risk (Harding \& Helweg-Larsen, 2008). Comparatively, actual re-assault (physical, verbal or sexual) is a more quantifiable measurement for concrete assessment of reduced or increased safety. The most effective tools likely measure all of the above, but research has not yet discovered how risk accuracy can be predicted among victims (Cattaneo et al.).

For the majority of women who are prone to remain in their relationships, reports of utilizing safety strategies can shed light on the effectiveness of such strategies (Parker \& Gielen, 2014) and especially when coupled with reports of perceived benefits (Appollis, Lund, de Vries \& Mathews, 2015). Some tactics for mitigating abuse among those who remain in abusive relationships are: "negotiating with the abuser, making the abuse more visible and more likely to be 
sanctioned, sanctions by family or friends, and/or police arrest leading to court sanctions" [...] and voluntary counseling" (Dienemman et al., 2002, pg. 223).

Measuring re-assault as a method for quantifying safety has been employed by several sources. Despite the accuracy of this approach, the task itself presents logistical problems such as long follow-up periods, relying on collateral or retrospective information, and no definitive cut-off point for when it is safe to say violence did not reoccur as it could again any time indefinitely into the future (Bowen, 2011). Yet, such measurements do tell some of the story of participant safety. One study using this approach with South African women being tested for HIV in antenatal clinics found that a 20 minute safety planning session "contributed to a significant reduction in the level of IPV" with a "mean danger assessment score of 6.0 before intervention [falling] significantly to 2.8 after 3 months" (Matseke et al., 2013, pg. 40). However, such assessments require either alterations of measurement tools, given that many tools focus on assault in the last year, or longitudinal design (Wagman et al. as cited in Kennedy et al., 2015). Nonetheless, it has been shown that reports of safety are consistent between two days, and 2, 6 and 12 week follow-up periods, suggesting that short-term follow-up may promise the same results as longerterm follow-up (Kendall et al., 2009).

Feasibility, Acceptability and Satisfaction

The components of feasibility, acceptability and satisfaction were included as a means for evaluating the intervention and the implementation process. These components are typically included in small preliminary pilot studies. 
However, this study was more comprehensive than a pilot study and included these components for reasons specific to the nature of the study as a Phase II trial gathering data necessary to build a Phase III trial. Described in greater detail in Chapter Three, this study included these components because A. It is recommended that research on sensitive subjects such as IPV evaluate the effects of study participation on participants; and B. This study built a new approach into an existing program that has already been shown to be effective in previous studies. Therefore, this study sought to refine current services and monitor the effects of doing so by providing insight into the acceptability, feasibility and satisfaction of including an IPV component alongside mobile HCT. These evaluation components were reviewed only for the inclusion of the IPV component of the protocol (risk assessment and safety planning) as this information has already been provided for the HCT component of the protocol in past research. Implications from this study will be used to refine standard practices that address the HIV-IPV intersection and inform future research on the topic.

A literature review found recommendations for ethically conducting research on sensitive subjects such as HIV and IPV. Three themes were observed as recommendations for evaluating the effects of participation: studies should consider risks, benefits and harm of participation (Appollis et al., 2015). These categories were evaluated through questions gauging feasibility, satisfaction and acceptability related to the IPV component. Measuring Feasibility of the IPV Component 
One of the main ways the impact of IPV services has been measured is through assessment of protocol feasibility. Literature was found on some past experiences of implementing both risk assessments and safety plans, and this helped to inform appropriate research questions.

For risk assessment feasibility, past research on the Danger Assessment (DA) as a guide for safety planning found no adverse occurrences were experienced as a result of study involvement (Bloom et al., 2014). Another study, using a safety decision aid similar to the DA, quoted that the aid had helped reduce "decisional conflict after only one use in a racially and ethnically diverse sample of abused women" (Glass et al., 2010, pg. 959).

Related to safety planning, feasibility considers the ability for providers to implement them, participants reported ability to successfully use techniques from the plan (Lewis et al., 2015), and any risks encountered in the process (Appollis et al., 2015). Empirical support for implementing safety plans with diverse populations was found, with one source reporting successful implementation was not impacted by demographic characteristics such as "age, race, income, or length of victim-perpetrator relationship" (Kendall et al., 2009, pg. 290). The helpfulness of safety planning has been shown to be unaffected of the mode of administration (in-person compared to telephonic) and is achievable after only brief intervention periods (Bennett, Riger, Schewe, Howard \& Wasco, 2004) following short training periods for those implementing them (Saunders, Holter, Pahl, Tolman \& Kenna, 2005). Studies have positively reported on the feasibility of using strategies from safety plans; one source reported $75 \%$ of women 
developed an emergency escape strategy during safety planning, and indicated that most women successfully "employ components of their safety plan in order to become safer" (Goodkind as cited in Parker \& Gielen, 2014, pg. 590).

Thus, the overarching point of assessing the feasibility of an IPV approach is two-fold: determining how feasible the techniques are to use and any harms experienced as a result of study participation. Questions over the feasibility of using strategies or the plan as a whole can be gleaned from the above examples. Appropriate ways to measure perceived harm or risk of participation were garnered from the Appollis et al. (2015) systematic review and include questions over any "negative emotions... unwanted thoughts, distress, bother, or drawback" (pg. 11). Finally, a way to measure the risk of using techniques or the plan was identified as simply asking about any negative occurrences experienced as a result of using the strategies (Bloom et al., 2014).

Measuring Acceptability of the IPV Component

Another important way to measure the impact of IPV services is to assess reported acceptability among participants. Information from past research on acceptability suggests including questions probing the helpfulness of services. Lewis et al. (2015) defines acceptability as participants finding the intervention to be "agreeable, palatable, or satisfactory" (Lewis et al., 2015, pg. 5), and another source explains it considers how participants respond to it (Bowen et al., 2009, pg. 3). Past investigation showed women usually describe safety planning as the single most helpful factor in reducing IPV (Bloom et al., 2014), with $90 \%$ of participants finding IPV screening itself to be acceptable (Thackeray et al., 2007). 
More, studies on sensitive subjects have found that participants with a history of abuse are more likely to find study participation beneficial, and this may be attributable to the catharsis experienced when discussing relationship difficulties to an empathic listener. Yet, most research in this area has failed to ask participants about perceived benefits (Decker et al., 2012). Overall, questions probing for perceived helpfulness of a protocol as well as the benefits of participating in a study over abuse are both important areas to consider when assessing acceptability among people with IPV.

\section{Measuring Satisfaction of the IPV Component}

The final area to be included when measuring the impact of IPV services is participant satisfaction. While there are several ways to measure satisfaction, approaches such as conducting risk/benefit comparisons were shown to help assess overall satisfaction of study participation. This is different than acceptability of the actual components of the protocol because this focuses on satisfaction associated with study participation as a whole. Some areas considered were general questions about whether or not services were helpful overall (Decker et al., 2012) and any regrets of participating in the study or talking about the issues at hand during the assessment process (Appollis et al., 2015).

In total, literature demonstrates that the effectiveness of safety planning is not only measured by the ability to use its techniques and the danger experienced while using it, but also it is important to consider any distress or difficulties experienced in the process of implementing a risk assessment and 
safety planning protocol. The culmination of participant responses, for an overall evaluation of experience, is necessary for creating a complete risk to benefit assessment of study involvement (Appollis et al. 2015).

\subsection{Summary}

This chapter has explored the study's Independent and Dependent Variables. Pertinent concepts have been operationalized, and empirical support has been provided from current literature to demonstrate how the protocol was developed. Theoretical frameworks have also been included to deconstruct the mechanics of the protocol. This chapter has paved the way for the reader to have a greater understanding of extant knowledge on the HIV-IPV intersection and the rationale behind the best practices incorporated into the protocol. Next, Chapter Three will review the study's methodology and provide further insight into how these myriad concepts and theories worked together in the administration of the HIRS protocol. 


\section{CHAPTER 3}

\section{METHODOLOGY}

Chapter One outlined the breadth of the HIV-IPV intersection and how it has disparately scourged women in South Africa. Chapter Two provided theoretical and empirical support for the intervention as well as the rationale for measurement methods. Hitherto, the need for effective practices with this population as well as more rigorous research in low-resource countries, such as South Africa, has been underscored. This study tests a protocol designed to address the HIV-IPV intersection and does so by using a risk assessment and safety planning protocol to assist women with intimate partner violence (IPV) who have tested HIV+ during mobile HIV Counseling and Testing (HCT). Through the testing of this protocol, new knowledge has been created on behaviors and perceptions after mobile testing as well as the impact of the HIV-IPV intersection on linkage to HIV care.

This study was a Phase II trial administered to test the effectiveness and safety of an HIV-IPV risk and safety protocol as a means to inform a Phase III study at a later date. As an experimental study over the novel HIRS protocol, the primary aims were to test for protocol effectiveness with study hypotheses related to safety and linkage to care. Because the study refined a current 
evidence-based program, by adding the IPV component, secondary aims sought to determine the feasibility, acceptability and effectiveness of the IPV component. This chapter explains the study design and methodological procedures employed to collect adequate data to answer the study's research questions.

The following research questions were devised to address some of the gaps in the literature related to the HIV-IPV intersection.

\subsection{Research Questions \& Hypotheses}

\section{Primary Research Questions}

\section{Research Question 1A}

What is the difference in participant risk and safety scores between the combined Experimental groups and the Standard of Care group?

Hypothesis: Participants in the combined Experimental group (receiving any level of the intervention) will have improved scores on the HIV IPV Risk and Safety scale (HIRS) as compared to participants receiving only the standard of care. Research Question 1B

What is the difference in participant risk and safety scores between dosage levels of those who received 1 . danger indicator score + initial safety plan, and 2 . danger indicator score + initial safety plan + follow-up safety plan? Analysis was done on pre post difference scores.

Hypothesis: Participants with the highest level of dosage will have the highest amount of increased perceived safety as measured by the HIRS.

Research Question 2 
What is the difference in participant linkage success between the combined Experimental groups and the Standard of Care group? Successful linkage (within 30 days) and number of days linked were used as indicators.

Hypothesis: Participants in the combined Experimental groups (receiving any level of the intervention) will have increased rates of linkage (more links at a faster rate).

\section{Secondary Research Questions}

\section{Research Question 3A}

What is the difference in participant satisfaction scores between the combined Experimental groups and the Standard of Care groups?

Hypothesis: Participants in the combined Experimental groups (receiving any level of the intervention) will have higher satisfaction scores as compared to participants receiving only the Standard of Care.

Research Question 3B

What is the difference in participant satisfaction, feasibility and acceptability scores between dosage levels of those who received 1. danger indicator + initial safety plan, and 2. danger indicator + initial safety plan + follow-up safety plan? Hypothesis: The possible impact of higher dosage intervention on satisfaction, acceptability and feasibility is uncertain.

\subsection{Specific Study Aims}

Phase I of Pilot study: 
1. Refine study instrumentation via focus interviews; improve cultural sensitivity of instrument as well as feasibility of use (readability, problems with wording, etc.).

2. Refine program components via focus interviews; improve cultural sensitivity of safety plan (phrasing/wording).

Phase II of Pilot study:

1. Determine feasibility of safety planning protocol: A). successful implementation with $75 \%$ of eligible participants 1). participants are reachable via call centre; 2). Participants report using safety plan. B). successful retention of $75 \%$ of participants at one month follow-up.

2. Determine acceptability of safety planning protocol: A). $80 \%$ of participants have a positive rating of the intervention. B). Determine if participant acceptability varies between dosage levels and between experimental and control groups.

3. Test effectiveness of strategies used to avoid contamination of sample between control and experimental groups; successful avoidance of contamination determined by low contamination percentage of $10 \%$.

4. Determine effectiveness of intervention; A). effectiveness with participant safety; B). effectiveness with linkage to care.

\subsection{Design}

As an Experimental pre-test-post-test control group design, this study compares the findings of two intervention groups to those of a control group to 
test hypotheses (see figure 3.1). Aligned with the purpose of a Phase II clinical trial (National Institute of Health, $\mathrm{NIH}, 2008$ ), the study builds on the work of past studies and seeks to further test the effectiveness and safety of an HCT service that addresses an IPV component. Because the new IPV component was added to an existing evidence-based program, the feasibility of the new component was tested. Processes considered were feasibility, acceptability, and satisfaction with the overarching study purpose to test protocol effectiveness with linkage to care and participant safety. Feasibility components are used to help uncover challenges in managing a protocol, needed resources, and needed potency (dosage) of an intervention in order to achieve effectiveness (Thabane et al., 2010). Three groups were involved in this study: one as a Standard of Care group, which did not receive risk assessment scores or a safety plan, and two sub-groups within the Experimental group, which received different dosage levels of the experimental component as a means to test the needed level of intervention potency (dosage level 1 and dosage level 2).

Figure 3.1 Research Design

$\begin{array}{lllll}R & O_{1} & & & O_{2} \\ R & O_{1} & X & & O_{2} \\ R & O_{1} & X & X & O_{2}\end{array}$

\subsection{Data Analysis}

Independent sample $t$ tests and Chi square tests were largely employed during data analysis. T tests were used specifically to test for condition differences on safety scores (between each dosage level) on the HIV IPV Risk and Safety (HIRS) scale. Chi Square was most often used to test differences in 
Satisfaction, Feasibility and Acceptability item responses between the combined Experimental group and the Standard of Care group and between the Dosage level groups within the Experimental group. Pearson's Chi-square test was used to test group differences in successful linkage to care between the Standard of care group and the Experimental group, and pre/post differences for ordinal items were analyzed using McNemar's test. In the cases where there were less than 5 responses per cell, Fisher's exact test results were used to meet analytical assumptions (Faul \& Lawson, 2012; Rubin \& Babbie, 2008). Additionally, results for all analyses were reported using two-tailed tests of significance for $p$ values as a means for "testing non-directional hypotheses" (Rubin \& Babbie, 2008, pg. 510). Prior to conducting these analyses, reliability and validity testing was conducted over the HIRS pre-test scale as well as the Danger Indicator. Exploratory Factor Analysis was used for this analysis.

\subsection{Operational Definitions}

The Independent Variable was: Intervention with two dosage levels. The intervention was twofold: 1). Participant received risk assessment and is informed of Danger Indicator score; 2). Participant then developed a 7-domain safety plan to address personalized risks uncovered in risk assessment. Those in Dosage level 2 received an additional safety plan approximately two weeks after the initial intervention.

This intervention was created with the intention of addressing the HIV-IPV intersection among HIV testers, with the ultimate aim of promoting safe and 
satisfactory linkage to HIV care; hence, this was an HIV intervention that addressed IPV as a barrier for linkage to care following mobile testing. This protocol did not seek to be an IPV intervention, per se; instead, this intervention attempted to impact subjective norms at the individual level as a way to, not only, catalyze participants to link to care but to do so in the safest manner, given the IPV dynamic. However, the risk assessment and safety-planning component of the protocol was built from evidence-based practices found among the IPV knowledge base.

The two Dependent Variables for the primary research question were: 1). risk \& safety score, and 2). linkage rate. The Risk and Safety score was derived from answers to the HIV IPV Risk and Safety (HIRS) scale, which was created for this study and was 10 items. The linkage to care rate was operationalized as successfully linking to care within 30 days. However, those who took longer to link to care, but did so by post-test data collection, are shown separately in the Result's section.

The three Dependent Variables for the Secondary research question were: satisfaction, feasibility and acceptability. Satisfaction was measured using three items as separate indicators of satisfaction: 1). Overall, the information you received about relationships was helpful, 2). It was helpful to be asked about the difficulties in your relationship? and 3). You regret talking about the difficulties in your relationship?

Feasibility was measured in the four domains of 1). Time to reach, 2). Length of time to administer intervention, 3). Techniques employed, and 4). Risk. 
Domain one included the number of attempts it took to reach participants for each component of the protocol (pre-test for all groups, follow-up safety plan for dosage level 2 only, and post-test for all groups). Domain two calculated the amount of minutes it took to administer each component of the intervention (pretest for all groups, initial safety plan for both experimental groups, follow-up safety plan for dosage level two only, and post-test for all groups). Domain three considered the feasibility of using the safety plan or any of the safety strategies and included the item 1). Did you use the safety plan? And 2). then considered if participants reported having used any of the strategies from the plan. Finally, domain four focused on risks associated with study participation as well as use of safety strategies and included the following items: 1). If you notified your partner of your HIV status, did you experience any type of violence as a result (mental, physical or sexual)?, 2). Would it be safe for us to contact you by phone at one month, two months or three months? (yes to any= safe to contact again), 3). Talking about the difficulties in your relationship placed you at greater danger? And 4). The services you received placed you at greater danger?

Acceptability considered acceptability of the safety plan and any discomfort experienced as a result of participating in the study. This section included the following items: 1). Did you find the safety plan helpful?; 2). Would you recommend the safety plan for a friend?; and 3). Did the respondent use any type of safety strategy (not an item directly asked but created from the number of respondents reporting use of at least one safety strategy) and 4). It was uncomfortable for you to discuss the difficulties in your relationship? 
Informed by the Appollis et al. (2015) systematic review on conducting research on violence and abuse, these items were included to measure participant experience of participating in the study as well as utilizing the techniques promoted within the intervention. This source highlights the importance of using items that probe for perceived feasibility, acceptability and satisfaction of any study over the sensitive subject of abuse. By analyzing each of these areas as separate indicators of participant experience, a study may procure more meaningful data over perceived benefits, harms and risks of involvement in research about abuse. This is crucial for all research about abuse given that there is the potential for questions to trigger traumatic memories. However, Decker et al. (2012) found that respondents who have a history of abuse report finding studies about abuse more acceptable (than their counterparts who have not endured abuse) and may even find the process therapeutic. Further information on these items used as separate indicators may be found in Chapter Two as well as Chapter Four.

\subsection{Participants}

Mobile HIV testers were invited to participate in this study if they met the study inclusion criteria. Foremost, they had to be 1) female and 2) have tested HIV+ during mobile HIV Counseling and Testing (HCT) with the chosen NGO in Gauteng province, South Africa. They had to 3) give written and verbal consent to participate in the study and 4) provide a reachable number for the call centre research team to reach them for telephonic study administration. Participants had 
to 5) currently (or often) be in a romantic relationship, 6) meet IPV eligibility (described below in this chapter), 7) be at least 18 years of age (or older), and 8) be able to participate in one of the chosen language of English, Sesotho, isiZulu or isiXhosa. Those who could not fill these inclusion criteria, or 9) who had cognitive impairment that precluded them from giving informed consent, were not eligible to participate in the study.

\section{Recruitment}

This study recruited participants from the NGO, Shout-it-Now (S-N), which provides mobile HIV Counseling and Testing (HCT) services in the South African province of Gauteng. S-N provides education and screening to 70,000 youth each year through this mobile program. Although S-N offers free services to those aged $\geq 12$ years, only those participants' aged $\geq 18$ years were approached for study participation. At this phase of the study, individuals were asked to participate only if they tested HIV+, were female, and could participate in one of

the four chosen languages. Those who agreed verbally and in writing to the informed consent process were then referred to the study where they were later screened by the research team for relationship status, IPV eligibility, access to a telephone and cognitive impairment.

Compensation

Participants were informed that by participating in the study, those who remained in the study throughout its entirety would have their name entered into a drawing for an i-pod. All participants received free HIV/TB screenings and 
counseling as well as a free IPV screen. Those who preferred not to participate also received the free HIV/TB screenings and counseling but no IPV screen.

\section{IPV Eligibility}

IPV Eligibility was established by answering either $\geq 2$ on the revised Nonviolent Control scale (NVC) or $\geq 1$ on the revised Conflict Tactics Scale 2 (CTS2) (see Table 3.1). Once eligibility was established, participants were immediately allocated into one of the treatment groups, but all received the remainder of the pre-test items. The eligibility items (the NVC and CTS2) in conjunction with the remaining pre-test scales (VVS, HIRS and DA) collectively made up the full pretest.

\subsection{Description of Instruments \& Rationale for Use}

\begin{tabular}{|l|l|l|l|}
\hline \multicolumn{2}{|l|}{ Table 3.1: Instruments \& Program Components } \\
\hline Dimension measured & Variable or scale name & Description & $\begin{array}{l}\text { Measurement within } \\
\text { study }\end{array}$ \\
\hline $\begin{array}{l}\text { Relationship status; Collected } \\
\text { in eligibility screening }\end{array}$ & $\begin{array}{l}\text { Relationship } \\
\text { Eligibility }\end{array}$ & $\begin{array}{l}\text { 2 items; one } \\
\text { minute or less. }\end{array}$ & $\begin{array}{l}\text { Dichotomous } \\
\text { (yes/no) }\end{array}$ \\
\hline $\begin{array}{l}\text { Danger related to Intimate } \\
\text { at Pre-test }\end{array}$ & $\begin{array}{l}\text { Danger } \\
\text { Indicator* (DI): } \\
\text { Composite Score of } \\
\text { CTS-2 (10 items); NVC } \\
\text { (6 items); VVS (8 } \\
\text { items); HIRS (10 } \\
\text { items); and DA (11 } \\
\text { items). }\end{array}$ & $\begin{array}{l}45 \text { items; About } \\
10-12 \text { minutes }\end{array}$ & $\begin{array}{l}\text { All, but VVS, } \\
\text { dichotomous with the } \\
\text { VVS being a 0-2 } \\
\text { likert scale; Score } \\
\text { range 0-49. }\end{array}$ \\
\hline $\begin{array}{l}\text { Physical and sexual violence; } \\
\text { Part of Pre-test \& eligibility } \\
\text { screening }\end{array}$ & $\begin{array}{l}\text { *Revised Conflict } \\
\text { Tactics Scale } \\
\text { (CTS2)(Straus, } \\
\text { Hamby, Boney-McCoy } \\
\text { \& Sugarman, 1996) }\end{array}$ & 10 items & $\begin{array}{l}\text { All dichotomous } \\
\text { (yes/no); Score } \\
\text { range 0-10. }\end{array}$ \\
\hline $\begin{array}{l}\text { Nonviolent control } \\
\text { (psychological/emotional } \\
\text { abuse); Part of Pre-test and }\end{array}$ & $\begin{array}{l}\text { *Revised Non-violent } \\
\text { control } \\
\text { (NVC)(Johnson \& }\end{array}$ & 6 items & $\begin{array}{l}\text { Dichotomous } \\
\text { (yes/no); Score } \\
\text { range 0-6. }\end{array}$ \\
\hline
\end{tabular}




\begin{tabular}{|c|c|c|c|}
\hline eligibility screening & Leone, 2005) & & \\
\hline $\begin{array}{l}\text { Violence vulnerability (risk } \\
\text { and protective factors); Part } \\
\text { of Pre-test }\end{array}$ & $\begin{array}{l}{ }^{*} \text { Revised Violence } \\
\text { Vulnerability Scale } \\
\text { (VVS)(Van Zyl, Brown } \\
\text { \& Pahl, 2013) }\end{array}$ & 8 items & $\begin{array}{l}\text { 3-point likert scale } \\
(0,0,1 \text { points } \\
\text { possible); Scale } \\
\text { range } 0-8 \text { (lacking } \\
\text { vulnerability-most } \\
\text { vulnerable). }\end{array}$ \\
\hline $\begin{array}{l}\text { Risk and safety related to the } \\
\text { HIV IPV intersection; Part of } \\
\text { Pre-test }\end{array}$ & $\begin{array}{l}\text { *HIV IPV Risk \& } \\
\text { Safety (HIRS) scale } \\
\text { (created for this study) }\end{array}$ & 10 items & $\begin{array}{l}\text { Dichotomous } \\
\text { (yes/no); Score } \\
\text { range } 0-12 \text { (no risk- } \\
\text { most risk) }\end{array}$ \\
\hline $\begin{array}{l}\text { Intimate partner violence } \\
\text { lethality level; Part of Pre-test }\end{array}$ & $\begin{array}{l}\text { *Revised Danger } \\
\text { Assessment (DA) } \\
\text { (Campbell et al., 2009) }\end{array}$ & 11 items & $\begin{array}{l}\text { Dichotomous } \\
\text { (yes/no); Score } \\
\text { range 0-13 (limited } \\
\text { lethality-highest } \\
\text { lethality) } \\
\end{array}$ \\
\hline $\begin{array}{l}\text { Feasibility; Part of Post-test } \\
\text { for Experimental groups only }\end{array}$ & $\begin{array}{l}\text { Feasibility: items } \\
\text { used as separate } \\
\text { indicators }\end{array}$ & 5 items & $\begin{array}{l}\text { Dichotomous } \\
\text { (yes/no) }\end{array}$ \\
\hline $\begin{array}{l}\text { Acceptability; Part of post- } \\
\text { test for Experimental groups } \\
\text { only }\end{array}$ & $\begin{array}{l}\text { Acceptability: items } \\
\text { used as separate } \\
\text { indicators }\end{array}$ & 2 items & $\begin{array}{l}\text { Dichotomous } \\
\text { (yes/no) }\end{array}$ \\
\hline $\begin{array}{l}\text { Satisfaction level; Part of } \\
\text { Post-test for All groups }\end{array}$ & $\begin{array}{l}\text { Satisfaction: items } \\
\text { used as separate } \\
\text { indicators }\end{array}$ & 3 items & $\begin{array}{l}\text { Dichotomous } \\
\text { (yes/no) }\end{array}$ \\
\hline $\begin{array}{l}\text { Linkage to care; Part of Post- } \\
\text { test for All groups }\end{array}$ & Linkage to Care & $\begin{array}{l}\text { Linkage } \\
\text { status and } \\
\text { rate }\end{array}$ & $\begin{array}{l}\text { Dichotomous: Link } \\
\text { (yes/no); } \\
\text { Rate (within } 30 \\
\text { days and by post- } \\
\text { test) }\end{array}$ \\
\hline
\end{tabular}

Note. Table 3.1 provides description of each item included in study's instrumentation.

The Non-violent control scale (NVC) measures psychological maltreatment of women and is used to differentiate IPV typology such as Situational Couple violence and Intimate Terrorism. This scale was originally part of the National Violence Against Women Scale (NVAWS), included 7 items with dichotomous (yes/no) responses and had a Cronbach's alpha of .70 (Johnson and Leone, 2005). The scale has not been used in Africa but does have support for international use. Findings from this study will contribute to the IPV knowledge base by applying Johnson's theory in an area where it has not yet been applied. 
This study revised the original NVC by only using 6 items and changing some of the wording slightly.

The original Conflict Tactics Scale 2 (CTS2) measures various dimensions of IPV and includes symmetrical responses from both victim and perpetrator. Responses are elicited on a 7-point scale with 0 being "this has never happened," 1 being "once in the past year" ... and so on, with 6 being (the highest) "More than 20 times in the past year" and 7 being "Not in the past year, but it did happen before (Straus et al., 1996). Jones et al. (2003) recommends this scale for use when jointly working with HIV and IPV. Different versions of this scale have been used in sub-Saharan Africa (Hung, Scott, Ricciotti, Johnson \& Tsai, 2012; Maman et al., 2010; Zacarias, Macassa, Svanstrom, Sores \& Antai, 2012), and it has been specifically used in South African studies (Peltzer, 2013;

Swart, Seedat, Stevens \& Ricardo, 2002). The Physical Aggression dimension of the scale has 12 items and has produced a Cronbach's alpha of .86. The Sexual coercion dimension of this scale has 7 items with a Cronbach's alpha of .87. This study revised the original CTS2 so that it included items that measure physical ( 7 items) and sexual (3 items) abuse, was only administered to victims, and used dichotomous responses to the items (yes/no). Although it had previously been translated into isiZulu, items were re-translated for this study because of wording changes for this revision.

The Violence Vulnerability scale (VVS) measures if a romantic relationship is vulnerable for IPV to occur. This scale considers if participant's attitudes and beliefs place them at risk for IPV, and the scale was originally part of a study in 
South Africa regarding young men and women undergoing mobile HIV Counseling and Testing (HCT) in Gauteng or Limpopo provinces. Findings over this scale have not yet been published. It was originally an 11-item scale with a Cronbach's alpha of .81 and a Corrected Mean total correlation of .45.

Responses were elicited on a 3-point scale with 1 being "Almost never or never," 2 being "Once in a while," and 3 being "Frequently" (Van Zyl et al., 2013). This study revised the original VVS so that it only included 8 items, and points (one each) were only assigned to the "Frequently" category.

The HIV IPV Risk \& Safety scale (HIRS) measures perceived risk and safety factors associated with help-seeking behaviors for those affected by both HIV and IPV. This scale was created for this study and underwent validity and reliability testing after data collection. The pre-test and post-test versions have slight language variations that allow the pre-test to be seen as perceptions of what might occur in future attempts to access HIV care, and the post-test may be seen as a tool for reflecting on perceptions of danger for what actually occurred in the time between the pre and post-test. The scale elicits dichotomous (yes/no) responses and includes 10 items with the last item having 3 points assigned for affirmative responses (all other items are only assigned 1 point).

The Danger Assessment (DA) measures IPV lethality risk. The scale contains 20 items with weighted categories and involves the use of a one-year calendar to track the frequency of endorsed items. Scores from the scale are used to predict respondent's risk for IPV lethality and include categories (from lowest to highest) of variable danger, increased danger, severe danger, and 
extreme danger. This scale has had a test-retest reliability ranging from .89 to .94 (Campbell as cited in Campbell et al., 2009, pg. 659). A systematic review found that the DA had an Area Under the Curve (AUC) of .618 and a small effect size (the AUC is used to assess how predictive a risk assessment is of future reassault); however, the systematic review also found that most studies had implemented the DA incorrectly. When predicting femicide, the AUC of the DA was .916, and .674 "when predicting severe reassault not controlling for protective actions taken by the victim, and an AUC of .687 for severe reassault when controlling for victim protective actions" (Campbell et al., 2009 \& Campbell et al., as cited in Messing \& Thaller, 2013, pg. 1543). Past studies have found convergent construct validity of the DA was supported with moderate to strong correlations with other validated scales such as the Conflict Tactics Scale (Campbell as cited in Campbell et al., 2009 pg. 659). The DA has been used in South Africa in a healthcare study, showing a retention rate of $52.5 \%$ (Matseke et al., 2013), and a study over the use of orders or protections for IPV (Peltzer, Pengpid, McFarlane \& Banyini, 2013). One study raised concern over its validity in South Africa (Joyner \& Mash, 2012) when used merely as a safety planning tool and thought it was more accurate to use it as a "danger assessment" that should be further evaluated. This study revised the DA so that it only included 10 items and did not use the calendar to track frequency of endorsed items (given the nature of the telephonic version of this study, but see recommendations for future studies regarding the calendar use). Minor changes were made to the 
language of these items and input from the author of the DA was sought and honored in the final version of the instrument.

Rationale for items included on feasibility, acceptability and satisfaction are outlined in Chapter Two under Dependent variable.

$$
\text { Pre-test }
$$

The pre-test was comprised of 45 items total. This included the Nonviolent control scale, which measured the variable of non-violent control or psychological abuse related to systemic power and control; revised Conflict Tactics Scale 2 (CTS2), which measured the variables of physical and sexual abuse; revised Violence Vulnerability Scale (VVS), which measures violence vulnerability; the HIV IPV Risk \& Safety scale (HIRS), which measures risk and safety associated with the HIV-IPV link; and the revised Danger Assessment (DA). All 45 items were asked of all participants during telephonic contact.

\section{Post-test}

The post-test was comprised of the HIRS scale and many different items that were used as separate indicators of linkage to care and the components feasibility, acceptability and satisfaction. Post-tests varied slightly depending on group allocation. All participants received the 17 items of the post-test version of the HIRS, which included slight verbiage changes, as well as items on satisfaction. Only the experimental groups received items regarding feasibility and acceptability as these probed for responses to the risk assessment and 
safety planning component of the protocol. The post-test was also administered via telephonic contact.

\subsection{Intervention}

Participants in the experimental groups received two main interventions, with those in dosage level 2 group receiving more potency of the latter intervention. All participants in the experimental groups received both a Danger Indicator score and a personalized, 7-domain safety plan.

1. The Danger Indicator: The Danger Indicator includes all sub-scales in the pre-test with points assigned, according to the literature, to create a total score that is associated with a danger level. Participants were informed of their danger level (0-10, with 5 point increments), which corresponded with their total score. This instrument was created for this study and underwent reliability and validity testing as well as factor analysis to ensure that it contained one factor for which a global score could be assigned as a latent variable.

2. Safety Planning: Though safety plans are individualistic and a one-sizefits-all blanketed approach cannot be prescribed word for word, all participants received an initial safety plan, which followed a 7-domain format to elicit personalized responses for person-tailored plans. Participants in the dosage level two experimental group received a second safety plan, or a follow-up safety plan, to help participants revise their initial plan if anything had changed in the time between pre and post-test. The intent of the safety plan was to increase participant's overall safety, given that violence has been shown to increase after 
HIV diagnosis, and to help them link more safely to HIV care. Parker \& Gielen's (2014) six IPV safety-planning domains were used as a guide during this protocol and a $7^{\text {th }}$-domain was created for this study as a means to help with safe HIV help-seeking behaviors. The $7^{\text {th }}$ domain was informed by the NNEDV's power and control wheel, the work of Mkandawire-Valhmu et al. (2013), and insight from exploratory studies over women living with HIV and IPV in southern Africa.

The domains were as follows: 1. Formal network; 2 . Informal network; 3. Legal; 4. Placating strategies; 5 . Resistance Strategies (which are not encouraged but, instead, are discussed as a means to dissuade their use given that they are shown to increase violence); 6 . And general safety planning strategies, 7. Accessing HIV care safely. The content for each of these topic domains was tailored to address each participant's unique circumstances discussed in the call.

Goals for safety plan were: 1. Educate participants on the dynamics of IPV in their relationship as a means to alter subjective norms about gender-based violence, 2. Inform participants of strategies known to increase or decrease safety, and 3. Create a plan for ways to safely access medical services for HIV.

The safety plan was Psychoeducational in nature and not an IPV counseling session. The first intervention component informed women that the Danger Indicator instrument detected the presence of IPV in their relationship, and they were told their level of danger as related to a 10-point scale (with 10 being the highest level of IPV). They were then asked to reflect on what this information meant to them. Next, participants were educated about what types of 
behaviors were considered abusive from a theoretical level, and they were given empirical information about the likelihood of violence increasing after an HIV+ diagnosis. Safety strategies were discussed with each strategy followed by reflective questions. Participants were also informed of some strategies that have been shown to escalate violence. Participants were informed about the existence of IPV services in their area and were educated on legal options available as well as the process for utilizing legal services such as orders of protections (otherwise known as interdicts). Referrals for IPV and legal services were given for those who said they wanted them and strategies were discussed for safe places to keep the information away from the abusive partner. Participants were asked during the safety plan if they planned to leave the relationship, as this information was vital to informing the direction of the safety plan given that violence has been shown to greatly increase at such a time. However, those who were not planning to leave the relationship were supported in their decision and were not encouraged to leave. Finally, participants were asked to create a global safety plan and particular emphasis was placed on discussing safe ways they could link to care, take medicine or notify their partner of their seropositive status if desired. They were informed that partner notification of their serostatus was a personal decision (UNAIDS, 2000), as they knew what was best for their own lives and what was safest in their relationship.

Those in Dosage level two group received a follow-up safety plan which allowed the research team to review the participant's personal safety plan with 
them from approximately two weeks before. Participants were asked if anything had changed and if they needed any new information or resources.

\subsection{Procedure}

All participants underwent mobile HIV Counseling and Testing (HCT) and received the same HIV testing services. These services entailed that participants watched an edutainment video as a means to encourage testing uptake (Van Zyl \& Barney, 2014). Individuals, who met the criteria at this stage (had not yet been screened for relationship status or IPV eligibility), were invited to participate in the study. Written informed consent was obtained at this time in-person, and verbal consent was again obtained via the telephonic medium before the study was administered. A one-day lag time existed between diagnosis (HIV testing) and referral to the study. Therefore, the soonest a potential participant received contact from the research team was 24 hours after diagnosis/testing. One month after HIV diagnosis/testing, the research team began attempts to reach participants for post-tests.

Phase I of this study went approximately one month and was used to elicit feedback over the protocol. Participants referred to the study during Phase I were not screened for relationship status or IPV eligibility but were, instead, only asked to give feedback over the wording of the instruments, with particular attention paid to the cultural appropriateness and shared understanding of the language of the instruments. Once this phase was concluded, the feedback was used to 
improve the protocol and phase II commenced. A brief outline of the findings of this phase is presented in Chapter Four.

Phase II followed the same recruitment procedure above; however, once a prospective participant had agreed to participate in the study, she then was screened for IPV eligibility and relationship status. Both phases were administered telephonically. Once an individual had met all the criteria and written and verbal consent had been obtained, she was then assigned into a study group based on random allocation. The treatment groups and arms are described below:

\section{Standard Of Care}

Immediately following an $\mathrm{HIV}+$ diagnosis, all participants received information and referral to local mental health services. Given that WHO (2013) recommends that all healthcare workers responding to reported IPV provide at least first-line

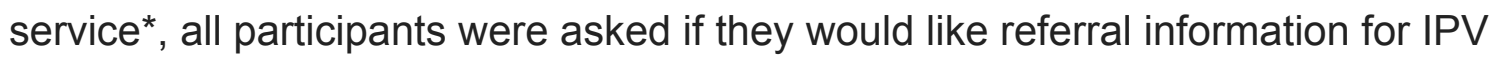
services before ending the pre-test phone call. Further, all participants were contacted again by the NGO's call centre program and again were offered referral for local mental health services if they were in crisis. *A detailed outline of first-line support can be found in WHO (2013), but a general description of firstline support

refers to the minimum level of (primarily psychological) support and validation of their experience that should be received by all women who disclose violence to a health-care (or other) provider. It shares many elements with what is being called 'psychological first aid' in the context of emergency 
situations involving traumatic experiences (WHO, 2013, vii).

Further, participants in this study group only received a pre-test and a post-test but were offered the same interventions as the experimental group after taking the post-test.

Dosage level 1

Participants received first-line support and were contacted for postdiagnosis support from the NGO's call centre. Participants in this study group received a score from the Danger Indicator as well as an initial safety plan.

Dosage level 2

Participants received the first-line support and were contacted for postdiagnosis support from the NGO's call centre. Participants in this study group received a score from the Danger Indicator, an initial safety plan, and a follow-up safety plan. Attempts to reach for the follow-up safety plan began two weeks from the referral date (HIV test date). This safety plan was used to update the initial safety plan if anything had changed for the participant in the time between the pre and post-tests.

Power analysis \& sample size

A power analysis showed that each treatment group would need at least 60 participants in order to have sufficient power for desired analytical methods. This breaks down to the following:

1. Experimental group: until 120 total (includes 75 each at $80 \%$ retention rate):

a. Dosage level one (60) 
b. Dosage level two (60)

2. Control group:

a. Standard of care (60)

\section{Randomization procedure}

The following randomization procedure was followed as a means for randomly allocating participants into one of the three group assignments. First a list of random numbers with a predetermined maximum $(75 \times 3$ or 225 in this case), without duplicates, was generated in Excel. This list was then copied into SPSS and three category codes were allocated to the random number representing the three conditions. When the study was extended to recruit a larger sample size, the same procedure was followed for another 125 randomized case assignments.

\section{Data Collection}

As outlined above, data collection for all participants began as soon as 24 hours after an individual underwent HCT and received an HIV+ diagnosis. Attempts for post-test data collection began at 30 days after the HIV test date. Attempts for follow-up safety plans began two weeks after the test date. Even in the case where a participant was not immediately reachable for any of these collection points, the next stage of the study was attempted at the scheduled time with the HIV testing date used to signal data collection times.

Three research assistants collected data using paper participant packets organized by language and group type. All research assistants administered the study with participants from each study group, and assignment to a team 
member was based on language and availability of research assistant at the time the referral came to the team. The research team all worked in one physical location to ease utilization of the randomization chart and ensure no group allocation was issued twice simultaneously. Once data had been documented on the physical participant packets, information was entered into Excel by the Research Coordinator and scanned versions of the original document were archived for reference. No identifying information was listed anywhere on these documents, and participant packets were coded by case ID number (assigned chronologically based on when the referral came to the team) with the key to this system remaining in the research room and destroyed at study termination.

\section{Data Management}

Privacy was maintained by all cases receiving a case identification number and data being stored electronically by the research team in South Africa on a secure server. At the end of the accrual period, the de-identified data file was encrypted by the Research Coordinator and forwarded electronically via email to the study PI. The data was provided in Excel format in order to ease migration to SPSS for analysis. The data file received was stored on the PC of $\mathrm{PI}$, with access to the computer being password protected, and the PC being HIPAA compliant. A copy of original files was saved on a CD and stored in a locked cabinet in the Pl's office.

\section{Staff and training}

The staff members administering the HCT were already employed by the NGO and had previously been trained in administering HCT. However, UofL 
researchers met remotely with these staff members for a brief IPV training and overview. These staff members used a script to ask for study participation and were trained in appropriate ways to go over the informed consent document. Staff members were offered the chance to receive a bonus for those who referred the most participants to the study. Because there were two research teams administering $\mathrm{HCT}$, the highest referring staff from each team was offered a bonus to reward their recruitment work.

Research team staff members consisted of one Research Coordinator and three Research Assistants and were recruited using two main mechanisms: 1. University of Louisville researchers made contacts with Social Work faculty at University of Cape Town and University of the Western Cape and asked if they could post the positions internally so their students may see the opportunities, and 2. The positions were also posted on non-profit advertising sites in South Africa commonly used for staffing recruitment by Shout-it-Now (i.e. Gumtree.org, NGOpulse.org, etc.). One Research Coordinator was hired to oversee day-to-day management of the study, and three Research Assistants were hired. Two Research Assistants worked full-time (M-F 8:30am-4:30am) and the other Research Assistant worked only two days a week (T \& W 8:30-4:30). The parttime assistant was hired on a part-time basis due to her availability, but she was offered full-time work.

The position announcements advertised that staff must: be comfortable discussing content related to HIV status and intimate partner violence; have an attitude of gender equality and support women's ability to make decisions for 
themselves; be empathetic and show non-judgment when working with participants; demonstrate cultural competence; and be people oriented, selfmotivated and able to work independently. The announcement also stated that desired staff must be computer literate (Microsoft Office), with particular knowledge of Excel. Although there were no degree requirements, all staff possessed at least a Bachelor's degree except for one who was in her final year of a Bachelor of Arts in Criminology.

The Research Coordinator was required to have research and management experience. She was Zimbabwean and possessed a Bachelor's of Arts in Education, a Master of Peace and Governance and was working towards a PhD in Conflict Transformation and Peace Studies. She was responsible for managing day-to-day procedures and supervising the overall study including staff performance and data entry.

All Research Assistants were required to have some type of experience comparable to HIV or IPV work and be fluent in English as well as one other language (between isiZulu, isiXhosa or Sesotho). All of the Research Assistants spoke fluent English, Xhosa and isiZulu with one also speaking Sesotho. All were South African nationality, and one had a Bachelor of Arts in Health Science and Social Services with a specialization in psychological counseling, and another a Bachelor of Social Sciences majoring in Psychology Criminology \& Sociology.

During the interview process, research team staff members were asked screening questions about attitudes and beliefs of gender equality in order to probe for any attitudes that would not be aligned with the nature of this study. 
Only staff with attitudes of gender equality were hired for this study, and the research team was all female. In order to gain an understanding of changes in staff attitude and beliefs of gender equality as well as staff knowledge on IPV \& HIV, research team staff members were issued a pre/post knowledge test (before any training began and after all training had concluded). There were 20 questions on this staff knowledge test and results were as follows. For the pretest, staff scored a mean of 50\% (10 questions correct out of the 20). For the post-test, staff scored a mean of $80 \%(16 / 20)$. Results showed a $30 \%$ improvement from entry-level knowledge.

Training consisted of four full days with an IPV-experienced Trainer from the United States and additional training time to complete CITI ethics training (Collaborative Institutional Training Initiative) online with University of Louisville. The Research Coordinator received on-going training through daily interaction with the main researcher of this study via Skype. Training included information sessions with the trainer over IPV, HIV the IPV-HIV link, information on HIV services offered by the NGO (presented by the NGO Director), philosophical discussions of ways to offer first-line support according to the WHO DV research recommendation, and extensive training on the study protocol including item by item reviews, discussions of translations, as well as role-play exercises and the shadowing of actual phone calls by the main researcher via Skype. All staff received a training manual, which was used alongside a power point presentation and handouts for training material. 
Prior to the implementation of the study, the main researcher and chair of the dissertation committee visited site locations. The main researcher visited site locations during the design stage of study development. First, areas in Gauteng province were visited to gain an understanding of the areas where mobile HCT is offered. The researcher also visited the study site at the NGO office in Cape Town to meet staff that assisted with HCT administration and understand the layout of the building for potential study implementation. Additionally, the chair of the dissertation committee conducted an onsite visit during the study's administration to address any questions staff may have had and to ensure that the facilities were conducive to collecting research data via telephone interviews.

\subsection{Ethical considerations}

This study was reviewed by the University of Louisville's IRB ethics committee and has been registered with National Health Research Ethics Council also known as the South African Department of Health (SANCTR), per the National Health Act, 2003 (Act No. 61 of 2003) and via the South Africa Human Research Electronic Application System. Finally, the creation and implementation of this study followed the WHO (2013) guidelines for Ethical Research on Domestic Violence Against Women and was informed by Jewkes, Watts, Abrahams, Penn-Kekana, \& Garcia-Moreno (2000) article Ethical and Methodological Issues in Conducting Research on Gender-Based Violence in Southern Africa. 
Documents were translated into each listed language by translators who had their credentials reviewed by University of Louisville's IRB committee. Documents were translated from English to each language and then backtranslated to confirm meaning. Translations were then reviewed with the research team to check for colloquial accuracy. 


\section{CHAPTER 4}

\section{RESULTS}

More research is needed on effective procedures with women who both are HIV+ and experiencing intimate partner violence (IPV); the need is especially critical in low resource countries such as South Africa where HIV rates and gender based violence are pervasive and those affected by the joint phenomenon suffer immensely. This study was designed to address the gaps of knowledge surrounding risk assessment and safety planning with women in South Africa who have recently tested HIV+ (in mobile HCT) and report IPV. The purpose of the study was to develop a valid and reliable psychometric instrument that assesses HIV-IPV-specific risk and determine the safety, effectiveness, feasibility, acceptability and participant satisfaction of a safety planning protocol that focuses on HIV-IPV-related danger levels. In addition to the main purpose of this study, a secondary aim was to also gain unknown knowledge regarding trends on perceptions and behaviors of those recently mobile-tested who have both HIV and IPV; all of which may be used to inform future research and practice that attends to the HIV-IPV intersection.

This study aimed to refine program components, retain all participants, avoid contamination, and test effectiveness of the intervention with participants' 
perceived safety and linkage to care. To improve the instrumentation and program components towards more cultural sensitivity, the study was administered in two phases. Phase I employed focus interviews to elicit feedback on instrument verbiage, with changes made to documents prior to the full protocol being administered. Phase II administered the HIV IPV Risk \& Safety (HIRS) protocol as a pre post design using randomized allocation into either a Standard of Care (SOC) group or one of two Experimental groups (together listed as combined Experimental group) which sought to test differences associated with protocol dosage level 1 and Dosage level 2).

Descriptive statistics, including frequencies and indicators of central distributions, were run to conduct data screening. Data were then inspected for outliers and missingness and subsequently cleaned. Intention-to-treat analysis was used for analytical procedures, and analyses over main research questions (hypothesis testing) reported two-tailed $p$ values for significance.

\subsection{Demographics}

A total of 446 people were recruited to participate in the program. Successful recruitment was defined as participants signing a consent form and having a reachable number. Of those 446, 191 did not report IPV sufficient for eligibility, 255 reported IPV and participated in pre-test data collection, and 249 of those were retained for post-test data collection. $99.97 \%$ were reachable at posttest and $97.65 \%$ were retained in the study by post-test. 
255 females participated in the study conducted between the months of July-December 1, 2015 (with Phase I conducted in June, 2015). After data cleaning and the removal of outliers, 248 participants were included in analysis. Of the 248 participants, 97 (39.1\%) were considered past testers (testing HIV positive previously (ranging from year 1996-2015), and the remainder (151; $60.9 \%$ ) were newly diagnosed as HIV positive during mobile HCT. All participants had an HIV test (regardless of their tester type (past tester versus newly diagnosed) within the time range of July 7,2015 - October 29,2015 . Of those past testers, most reported (qualitatively) having accessed mobile HCT services to gain their CD4 cell count. Of the $248,82(33.1 \%)$ were in the standard of care group, and 166 were in the experimental group. Of the 166 in the experimental group, $83(33.5 \%)$ received dosage level 1, and $83(33.5 \%)$ received dosage level 2. Participants all resided in Gauteng province, South Africa, identified as black South African, were currently in a relationship, and were of the age range $18-65$ with 33 being the mean age $(M=33.26, S D=9.89, M d n=32)$ but ages 25 , 26 , and 27 accounted for the highest percentages of the sample (18.6\%) and age 25 had the highest frequency (6.9\%). Chosen languages for study administration were: isiZulu: 176 (69\%); isiXhosa: 37 (14.5\%); Sesotho: 26 (10.2\%); English: 14 (5.5\%) [With 2 respondents having language unknown due to staff failure to record these $(.8 \%)]$. No other demographic information was collected. 
Figure 4.1 Participant Age

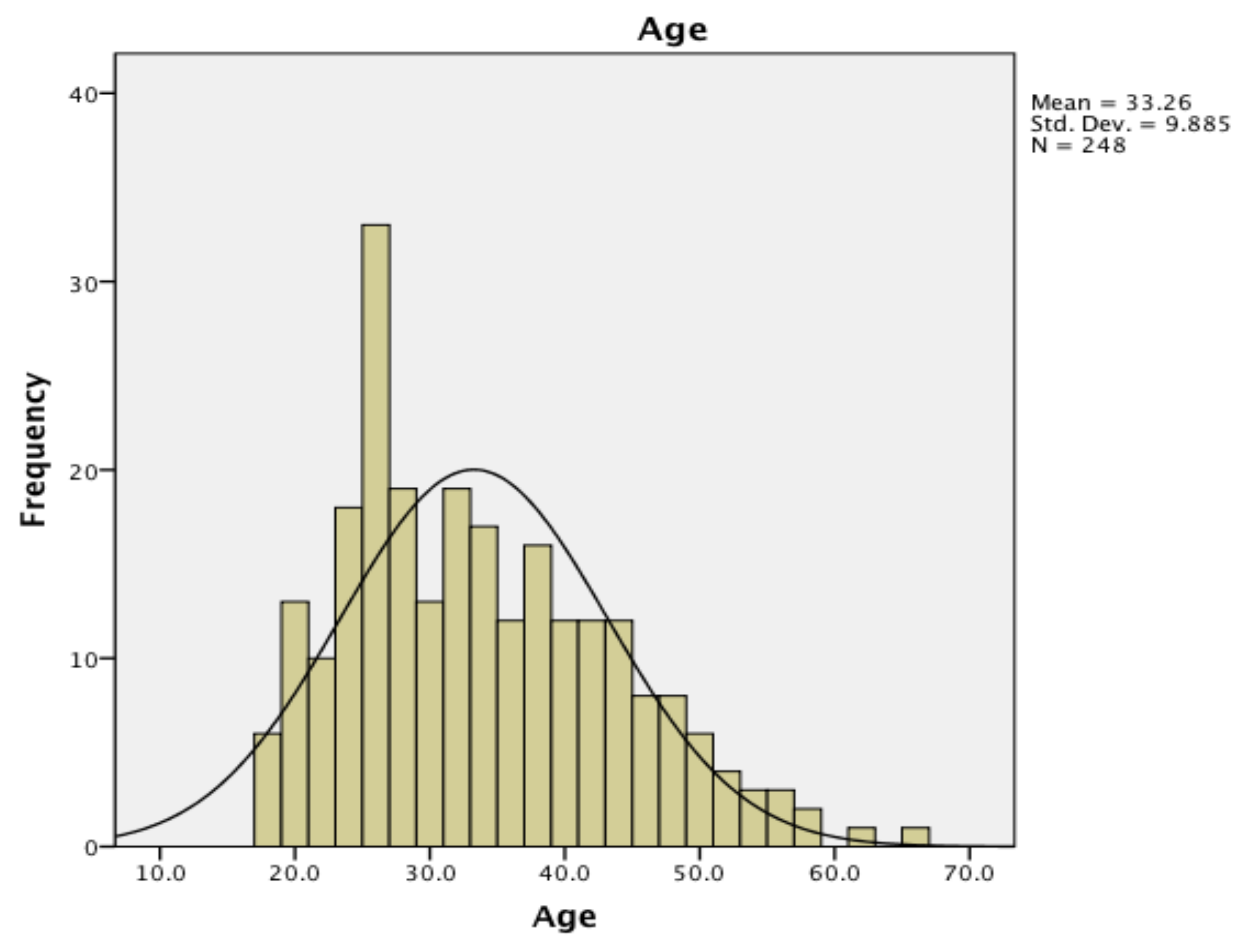

Figure 4.1. Distribution graph for total sample after outliers were removed $(n=248)$.

\subsection{Data Cleaning}

A total of 7 outliers were removed from general analyses with one removed from the Standard of Care group, 1 from the Experimental Dosage level 1 group, and 5 from the Experimental Dosage level 2 group. Reasons for removal were: cases with uncorrectable entry error from research staff $(2$ participants), and cases identified on multiple whisker plots as having issues such as exceptional length of time from referral to post-test or exceptional length of time from pre-test to post-test (5 participants). For questions relating only to post-test (linkage to care, safety, satisfaction) 3 cases were removed from the Standard of Care because they were contaminators (reported having accessed IPV services elsewhere in the last month, possibly from the first-line support 
referral). However, these three contaminating cases remained in the analysis for the item If you notified your partner of your status, did you experience any type of violence as a result? These were included in analyses for this item to consider how results of HIV-IPV-specific safety planning may differ from general IPV safety planning. Additionally, one person from the Standard of Care group was removed only for linkage to care comparisons due to a data entry error that could not be reconciled (linkage date was documented as one month before HIV testing date).

Six participants were lost to attrition, and thus were removed for any pre/post analysis but were included in pre-test only and descriptive statistics. Two of those lost to attrition were unreachable for post-tests and the remaining four opted to discontinue study participation. Information on those who dropped out was: most were past testers, all were younger than the mean age with a range of 24-31 $(M=28.2)$ and all were in Experimental groups, with most in the Dosage level 2 group (67\%) and remainder in Dosage Level 1 group. The mean Danger Indicator raw score for this group was $8(M=8$, with a range of $7-12)$ and most scored exactly a 7. Of those in Dosage Level 2 group, none received the follow-up safety plan, and only 3 of the 6 in the total group received the initial safety plan (3 reported it was not needed) and therefore would have only received the Danger Indicator score as their intervention.

Any analyses over post-test data only or analyses comparing pre-test with post-test results did not include those 6 participants who were lost to follow-up. Therefore, for these analyses, 242 total participants were included with 82 in the 
Standard of Care group, 81 in Dosage Level 1, and 79 in Dosage Level 2. The consort diagram below demonstrates these numbers.

Figure 4.2 Consort Diagram

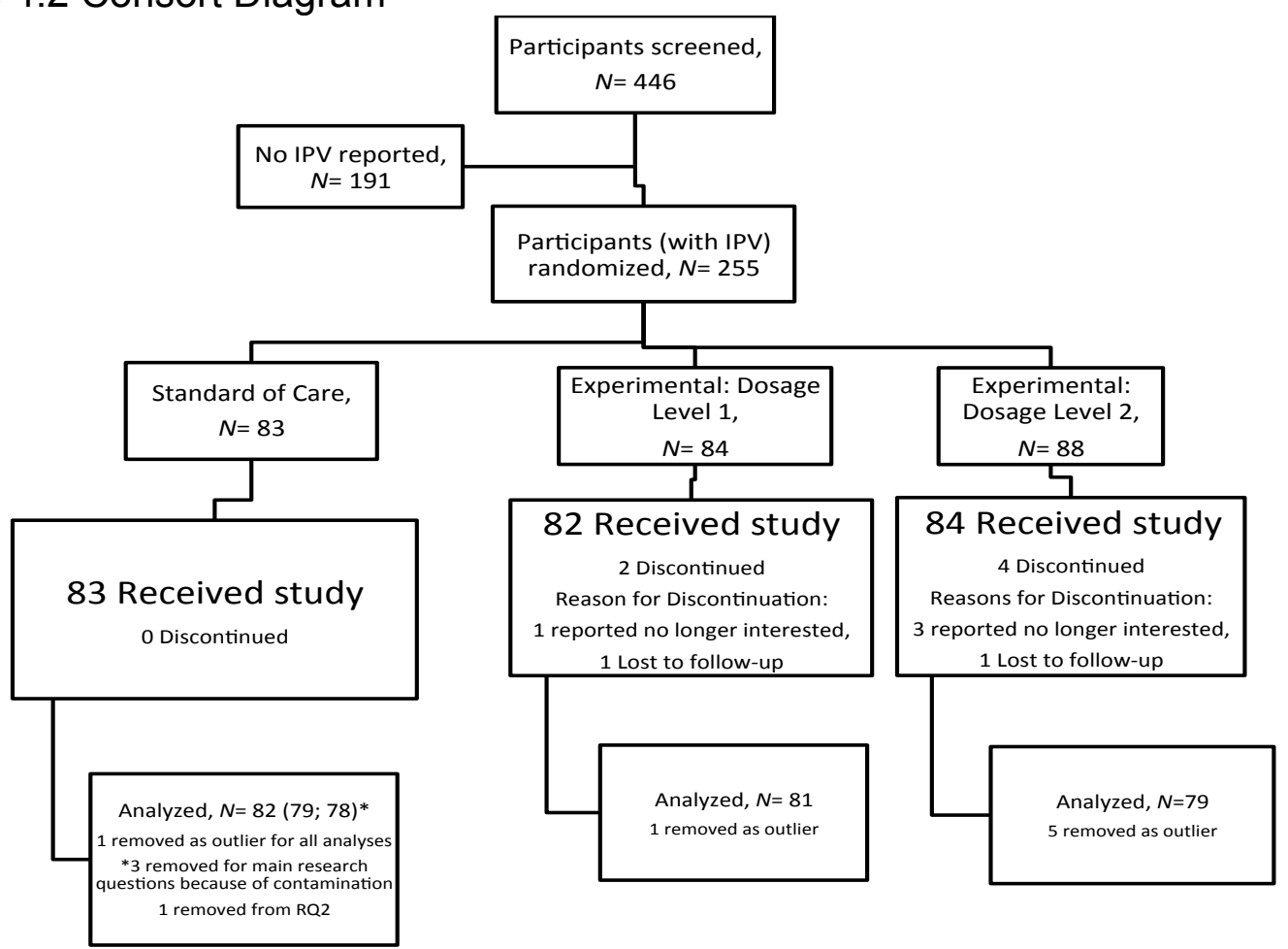

Figure 4.2. Consort diagram depicts amount of individuals agreeing to participant and the final numbers of those included in analyses.

\subsection{Reliability and Validity Testing of Instruments}

\section{Danger Indicator}

The psychometric instrument, the Danger Indicator (DI), was developed and tested for validity and reliability. This instrument was tested in pre-test scores of 255 participants. The DI consists of the following subscales: 6 items from the revised Non-violent control scale, 10 items from the revised Conflict Tactics Scale 2 (physical and sexual violence items), 8 items from the revised Violence Vulnerability scale, 10 items from the HIV IPV Risk \& Safety scale (HIRS), and 11 items from the revised Danger Assessment. Eligibility for the study, otherwise 
seen as IPV screening, was established by answering affirmative to $\geq 2$ items on the Non-violent control scale and/or $\geq 1$ items on the 10 -item revised Conflict Tactics scale (physical and sexual violence items) (Straus et al., 1996). Each of the subscales of the DI helps measure a component of a global indicator of danger.

Through reliability and validity testing and factor analysis, this DI was found to be an appropriate tool for measuring the construct of partner violence and particularly physical assault. An Exploratory Factor Analysis (EFA) using a Principle Axis Factoring method of the 45 items in the DI was conducted on the sample $(N=248)$ (with outliers removed).

The Danger Indicator is comprised of sub-scales, which were theoretically constructed to measure different sub-components of a wider domain. Because this study relied on the use of these scales for a global score, a factor analysis was done to see if the overall score was sufficiently unidimensional to justify using the total score as an indicator of danger. In order to do this, Principle Axis Factoring was used in exploratory factor analysis based on one factor to be extracted. One factor was extracted, requiring 4 iterations and explained $19 \%$ of the variance.

The item with the highest loading was the item "my body was hurt." This item may be seen as describing the core focus of the DI scale. Cronbach's alpha of this scale was .882 , which is quite high and indicates reliability sufficient to distinguish at the individual level various degrees of safety and danger. However, the validity for this scale is less than ideal. The mean of corrected item total 
correlation was .364 . Hence, the validity of the scale shows some limitation and future research should refine this instrument.

HIV IPV Risk and Safety (HIRS) scale

Cronbach alpha's for the HIRS (10 items) was .824 with a Corrected ItemTotal Correlation mean of .412 . Thus, this scale has good reliability and approaching sufficient validity. The full scale is listed Appendix D.

\subsection{Conceptual Definitions}

To measure Satisfaction, 3 items were used as separate indicators of satisfaction: 1. Overall, the information you received about relationships was helpful, 2. It was helpful to be asked about the difficulties in your relationship? And 3. You regret talking about the difficulties in your relationship? These items were created based on the Appollis et al. (2015) systematic review on the ethics of conducting research on sensitive research topics (determining risk-benefit ratio) and reflect two of the three general categories they used to measure the risks, harms and benefits of research about sensitive topics (risks are under the Feasibility component, and another harm question is under the Acceptability component). The item It was uncomfortable to be asked about the difficulties in my relationship could fall into this category as well (under harm) but was analyzed within the Acceptability component because discomfort does not preclude satisfaction (i.e. someone could experience discomfort while still being 
satisfied with a product overall, and some discomfort was expected given that the topic is inherently uncomfortable).

\section{Feasibility}

The Feasibility component of this study addressed four components of domains, with items within each domain being analyzed separately for various reasons.

Domain One measured the time it took to reach participants (number of attempts) and included the number of attempts for the pre-test and post-test for both groups and the follow-up safety plan for dosage level 2 only. Mean attempts were not appropriate for comparison considering that Dosage level 2 had an extra contact.

Domain Two measured the length of intervention administration and considered the time to administer the pre-test, initial and follow-up safety plans as well as the post-test.

Domain 3 focused on risks that may prevent implementation and included the item Did you use the safety plan? and compared the total number of strategies employed (see strategies under the full post-test instrument in Appendix I). Domain 4 measured four different questions related to risk: 1. If you notified your partner of your HIV status, did you experience any type of violence as a result (mental, physical or sexual)?, 2. Would it be safe for us to contact you by phone at one month, two months or three months (yes to any= safe to contact again), 3. Talking about the difficulties in your relationship placed you at greater 
danger? And 4. The services you received placed you at greater danger? All were analyzed separately because they measured different aspects of risk, mainly falling into the two categories of perceived risk or actual violence.

For example, the item If you notified your partner of your status, did you experience any type of violence as a result measures a behavior or actual violence that occurred. Whereas, the other items measured perception of risk and varying risk areas. The item Would it be safe for us to contact you again in one month, two months or three months asks the respondent to predict what would be safe for them in the future, and the items Talking about the difficulties in your relationship placed you at greater danger and The services you received placed you at greater danger ask the respondent to if they perceived the assistance received to have affected their safety levels in general.

When adding an IPV component to a protocol, it is essential to assess for any risks involved in implementation, as this information could be crucial to the safety of participants of future studies (Appollis et al., 2015). Reports of risk or harm may be a better indicator of overall participant safety because questions that measure subjective experience of safety can actually be measuring an increase in thoughts about the abuse rather than actual safety, especially among those with post-traumatic stress disorder (Cattaneo et al. 2007). However, in general, when individuals have a heightened perception that they are at risk, they are more likely to adopt protective behaviors to reduce the perceived risk, and this may be true for IPV victims as well. Harding \& Helweg-Larsen (2008) found that "perceptions of risk were associated with intended relationship decisions," so 
that women who perceived they were in more danger "exhibited greater intention to terminate" the relationship (pg. 9). Hence, heightened perception of risk could be indicative of changes in altered subjective norms about abuse or the person's contemplation of the acceptability of abuse in their relationship.

\section{Acceptability}

4 items were analyzed separately as indicators of Acceptability of this study and fell into the two categories of acceptability of the safety plan and any overall discomfort experienced with the protocol in total (all participants received the latter) 1. Did you find the safety plan helpful?; 2. Would you recommend the safety plan for a friend?; and 3. Did the respondent use any type of safety strategy (not an item directly asked but created from the number of respondents reporting use of at least one safety strategy) and 4. It was uncomfortable for you to discuss the difficulties in your relationship? The final question was included per the Appollis et al. (2015) Systematic review over questions to determine the riskbenefit ratio of research on relationship violence. Although higher correlation was expected between the Acceptability responses in the first category, reliability testing revealed there was low correlation among them. The second category was included to compare if those who received higher dosage levels experienced more discomfort. Crosstabs were run to investigate relationships between the items, using the item did you use the safety plan as a primary indicator. This was done to try and explain the phenomenon of behavior related to these items. For example, some respondents reported that although they did not use a safety 
strategy they would still recommend a safety plan for a friend. This example suggests that just because someone did not use the safety plan they would still find it acceptable for someone else. This could be indicative that the respondent finds the safety plan acceptable, in general, and that maybe there is another reason they personally did not find it helpful for their situation (i.e. their perception from the onset was that they did not need a safety plan). Hence, by analyzing these items separately and investigating comparisons between them, it becomes clear that these items measure different phenomenon or aspects of acceptability. The information gleaned from comparing these items will help clarify what components of the safety plan were acceptable compared to others.

Further, because the number of participants who reported poor acceptability was low in frequency (only $15 / 129$ reported the safety plan was not helpful), acceptability items were analyzed separately to assist with detecting differences that would have been more difficult when comparing a score of multiple items at once. Additionally, items were compared between dosage levels for the same rationale (to detect differences more accurately given the small number of individuals who found the protocol not helpful).

\subsection{Phase I Findings}

As previously stated, the purpose of Phase I of this study was to elicit feedback from participants over the appropriateness of the study instruments so that changes could be made prior to implementation. 10 potential participants were recruited for Phase I of the study, but only $40 \%$ of those were reachable 
and participated. Questions asked in Phase I focus interviews were intended to 1. Clarify meaning of language and vernacular appropriateness, 2. Elicit examples to confirm shared meaning of language, and 3. Probe for information for enhanced safety planning. None of the four participants interviewed were screened for IPV nor asked if they were currently in a relationship, and their feedback cannot be assumed to be representative of the population served by these instruments. However, it did provide some insight into cultural appropriateness and some changes were made to the instruments as a result of these interviews.

Focus interviews did encounter some difficulties that appeared to impede the quality of the interviews. Because the research room on-site at the NGO had not yet been set up (phones, computers, etc), these interviews had to be held via Skype (telephone only). Further, the full research team had not yet been hired, and so the interviews were held only in English. The Skype medium posed the problems of poor audio quality and the likely confusion of a non-local number listing (given the international Skype account used). Further, the Research Trainer was responsible for conducting Phase I, and she had an American accent that was likely a hindrance, given perceived or actual cultural differences assumed by participants due to the observed foreign accent and/or actual difficulty understanding one another's dialect/accent.

The decision to "confirm" a phrase or word was operationalized as at least half of respondents will agree with the language, or no more than 1 will disagree (the latter being important in cases where questions were not asked or answered 
of all participants). If the responses did not meet these criteria, the phrase or word was changed. 20 Phrases were confirmed for use through the interviews; 1 phrase ("partner") was confirmed but with the notation that it is important to tell each participant that the questions reference romantic partners (connecting the use of the term "partner" to the first question in Phase II "are you in a relationship," which was not asked of Phase I participants); and 1 phrase was changed from "follow-up" to "contact."

After these changes to the original instrument, translations were checked for accuracy and cultural appropriateness among the research team, who were each fluent in English as well as at least one of the other three languages. Minor changes were made to improve phrasing to fit better with the original English version of the instrument as well as the dialect. This language check was done as an effort to increase the research team's acuity in administering the instruments' reliably and was an important stage in refining the instruments for culture appropriateness, given that the focus interviews were only held in English.

\subsection{Phase II Findings}

\section{Study Aims}

Feasibility

Aim 1 of Phase II of the study focused on determining the feasibility of the protocol with successful implementation operationalized by $75 \%$ of eligible participants receiving the intervention, and $75 \%$ of participants retained at the 
one month follow-up. $98 \%$ received the intervention as assigned, $61.9 \%$ reported using the safety plan, and $97.65 \%$ were retained at the one-month follow-up.

\section{Acceptability}

Aim 2 of Phase II of the study focused on determining the acceptability of the protocol as operationalized by $80 \%$ of participants reporting a positive rating of the intervention, with attention to any variation between dosage levels and between combined experimental groups and the Standard of Care group. The first two items listed in Table 4.5 were used to determine positive rating. Both scores far exceeded the $80 \%$ mark, demonstrating the participants overwhelmingly reported a positive rating of the protocol. For the item Overall, the information you received was helpful, $92.47 \%$ of the total sample responded yes. $92.41 \%$ of those in the Standard of Care group responded yes to this item, and $92.5 \%$ of participants in the combined Experimental group responded yes. For the item It was helpful to be asked about difficulties in your relationship?, $97.07 \%$ of the total sample responded yes. $96.2 \%$ of those in the Standard of Care group responded yes, and $97.5 \%$ of those in the combined Experimental group responded yes. Differences between dosage levels are listed below in Table 4.6.

\section{Contamination}

Aim 3 of Phase II focused on avoiding contamination as operationalized by fewer than $10 \%$ of participants in the Standard of Care group report receiving 
IPV services from another source between pre and post data collection periods. The item used to determine contamination of the Standard of Care group was In the last month, have you gotten help for relationship abuse from any of the following: police, domestic violence hotline or program, counselor, pastor/spiritual leader or any where else? Only $4 \%$ of the Standard of Care group (3 participants) responded yes to this question. Therefore, it can be stated that contamination was successfully avoided. However, these 3 contaminating cases were removed from analyses in the situations listed above under data cleaning.

\subsection{Primary Research Question 1A}

What is the difference in participant risk and safety scores between the combined Experimental groups and the Standard of Care group?

Hypothesis: Participants in the combined Experimental group (receiving any level of the intervention) will have improved scores on the HIV IPV Risk and Safety scale (HIRS) as compared to participants receiving only the standard of care.

Prior to analyzing the difference in participant safety scores, scores for the group's pre-est were compared for equivalency in order to determine if the two groups were from the same population. An independent sample t-test compared the pre-test scores (HIRS with weighted scoring where all count as one point except item 10 which an affirmative response counts as 3 points) and results were non-significant $(t(1,237)=-.540 ; p=.589)$, indicating the two groups were sufficiently comparable at baseline to proceed with pre-post group difference analysis. The pre-test mean for the Standard of Care group was slightly lower 
than the mean for the combined Experimental group $(S O C=M=2.29, S D=2.74$, $S E=.31$; combined Exp.= $M=2.51, S D=2.97, S E=.24)$. Only participants who remained in the study for pre and post-tests were included in this analysis.

Table 4.1 HIRS Pre/Post \& Mean Difference Scores I

\begin{tabular}{lcll}
\hline Group & HIRS Pre-test & HIRS Post-test & $\begin{array}{l}\text { Mean Difference } \\
\text { (post-test - pre-test) }\end{array}$ \\
\hline Standard of Care & $M=2.29$ & $M=2.17$ & $M=-.13$ \\
& $S D=2.74$ & $S D=2.07$ & $S D=3.05$ \\
& $S E M=.31$ & $S E M=.23$ & $S E M=.34$ \\
\hline Combined Exp. & $M=2.51$ & $M=2.84$ & $M=.33$ \\
& $S D=2.97$ & $S D=2.17$ & $S D=3.07$ \\
& $S E M=.24$ & $S M E=.17$ & $S E M=.24$
\end{tabular}

Note. Table 4.1 depicts HIRS pre-test, post-test and mean difference scores between the Standard of Care and the combined Experimental group ( $N=239)$.

The mean pre-post difference score for the Standard of Care group was very similar to the mean pre-post differences score for the combined Experimental group $(S O C=M=-.13, S D=3.05, S E M=.34$; combined Exp. $=M=$ $.33, S D=3.07, S E M=.24)$. An independent sample t-test showed the pre-post difference scores between the Standard of Care and the combined Experimental group were non-significant $(t(1,237)=-1.09 ; p=.278)$. These results challenge the hypothesis that those in the Experimental groups would have a reduced mean score on the post-test compared to the Standard of Care group.

\subsection{Primary Research Question 1B}

What is the difference in participant risk and safety scores between dosage levels of those who receive 1 . danger indicator score + initial safety plan, and 2 . 
danger indicator score + initial safety plan + follow-up safety plan? Analysis was done on pre post difference scores.

Hypothesis: Participants with the highest level of dosage will have the highest amount of increased perceived safety as measured by the HIRS.

Table 4.2 HIRS Pre/Post \& Mean Difference Scores II

\begin{tabular}{lcll}
\hline Group & HIRS Pre-test & HIRS Post-test & $\begin{array}{l}\text { Mean Difference } \\
\text { (post-test - pre-test) }\end{array}$ \\
\hline \multirow{2}{*}{ Dosage level 1} & $M=2.48$ & $M=2.84$ & $M=.33$ \\
& $S D=3.02$ & $S D=2.25$ & $S D=2.92$ \\
& $S E M=.34$ & $S E M=.25$ & $S E M=.32$ \\
\hline \multirow{2}{*}{ Dosage level 2 } & $M=2.48$ & $M=2.84$ & $M=.33$ \\
& $S D=2.87$ & $S D=2.1$ & $S D=3.23$ \\
& $S E M=.33$ & $S M E=.24$ & $S E M=.36$
\end{tabular}

Note. Table 4.2 depicts HIRS pre-test, post-test and mean difference scores between dosage levels $(N=160)$

An independent sample t-test showed the mean pre-post difference scores between the experimental groups of Dosage level 1 and Dosage level 2 were non-significant $(t(1,158)=.009 ; p=.993)$. The two groups had almost identical mean difference scores $(D 1=M=.33, S D=2.92, S E=.33 ; D 2=M=.33$, $\mathrm{SD}=3.23, \mathrm{SE}=.36)$. These results challenge the hypothesis that participants with the highest dosage level will have the highest increase in perceived safety score and suggest that the extra dosage has no effect on participant perceived safety. Only participants who remained in the study for pre and post-tests were included in this analysis.

\subsection{Primary Research Question 2}


What is the difference in participant linkage success between the combined Experimental groups and the Standard of Care group? Successful linkage (within 30 days) and number of days linked were used as indicators.

Hypothesis: Participants in the combined Experimental groups (receiving any level of the intervention) will have increased rates of linkage (more links at a faster rate).

Table 4.3 Linkage to Care

\begin{tabular}{|c|c|c|}
\hline Group & Link to care by post-test & Link to care $\leq 30$ days \\
\hline Total & $109 / 238(45.8 \%)$ & $99 / 238(41.6 \%)$ \\
\hline Standard of Care & $32 / 78(41.03 \%)$ & $30 / 78(38.5 \%)$ \\
\hline $\begin{array}{l}\text { Combined Experimental } \\
\text { group }\end{array}$ & $77 / 160(48.13 \%)$ & $69 / 160(43.13 \%)$ \\
\hline
\end{tabular}

Note. Table 4.3 depicts linkage to care comparisons between the Standard of Care and the combined Experimental group $(N=238)$.

Figure 4.3 Link to Care by Post-test

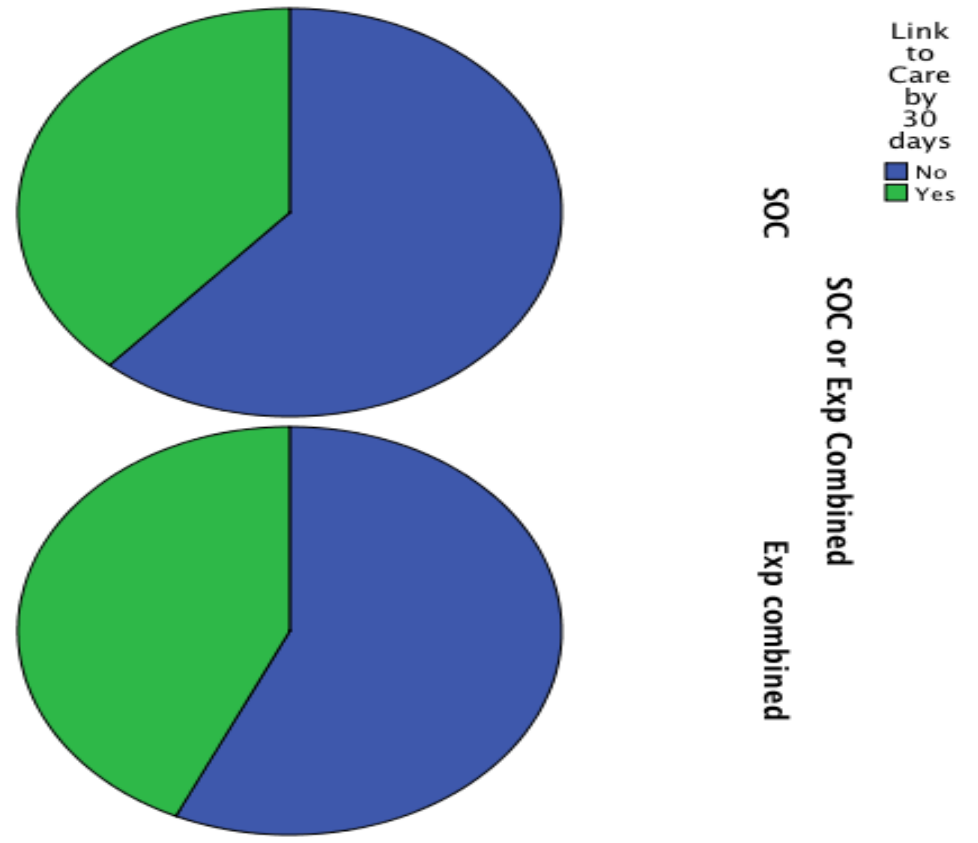

Figure 4.3 Percentage of individuals from total sample linked to care by post-test $(N=238)$. 
Figure 4.4 Link to Care within 30 Days
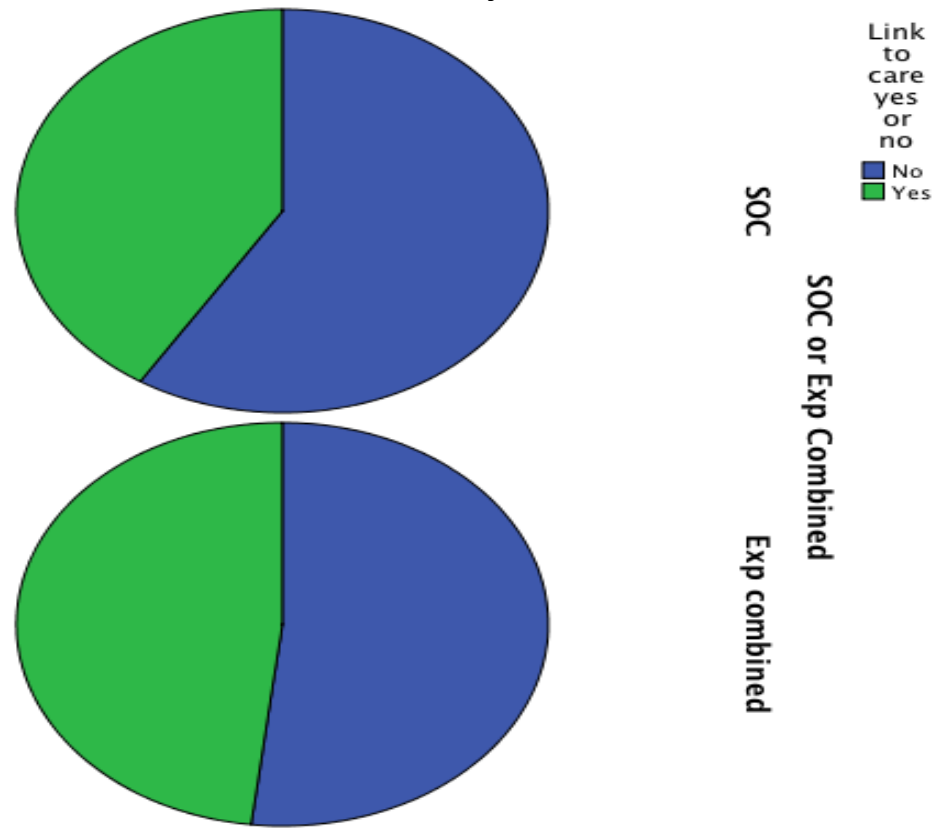

Figure 4.4 Percentage of individuals linked to care within 30 Days $(N=238)$.

A higher percentage of individuals linked to care, within 30 days, for the combined Experimental group compared to the Standard of Care group $(\mathrm{SOC}=$ 38.5\%; combined Exp.= 43.13\%). Several individuals in each group linked to care after the 30 day cut-off (SOC $=2$ linked $>30$ days; combined Exp. $=8$ linked $>$ 30days).

Pearson's chi-square test was performed to examine the relation between receiving the intervention and having a higher rate of linkage to care. Although a higher percentage of individuals in the experimental group linked to care, a chisquare test showed the difference in linkage success (link $\leq 30$ days) between the Standard of Care group and the combined Experimental group was nonsignificant $\left(X^{2}{ }_{(1)}=.471, p=.493\right)$. And the difference remained non-significant when comparing group differences in linkage by post-test $\left(X^{2}{ }_{(1)}=1.07, p=.302\right)$. It 
took slightly fewer days to link for the Standard of Care group compared to the combined Experimental group $(S O C=M=11.88, S D=11.45, S E M=2.02$;

combined Exp.= $M=13.43, S D=13.1, S E M=1.5)$, and the difference was nonsignificant $(t(1,107)=-.584 ; p=.560)$. The overall mean rate for linkage to care by post-test for the entire sample was 13.11 with $50 \%$ linking by 11 days $(M=13.11, S D=12.62, S E M=1.20)$ and only $9 \%$ linking after 30 days.

These results challenge the hypothesis that those in the combined Experimental group would significantly link at a faster rate.

Table 4.4 Linkage to Care by Age Group

\begin{tabular}{|c|c|c|c|}
\hline Age group & Total sample & Standard of Care & $\begin{array}{l}\text { combined Exp. } \\
\text { group }\end{array}$ \\
\hline \multirow[b]{2}{*}{$\leq 23$ years of age } & $14 / 39(35.9 \%)$ & $5 / 14(35.7 \%)$ & $9 / 25(36 \%)$ \\
\hline & $\begin{array}{c}2>30 \text { days }=16 / 39 \\
(41.03 \%)\end{array}$ & $\begin{array}{c}0>30 \text { days }=5 / 14 \\
(35.7 \%)\end{array}$ & $\begin{array}{c}2>30 \text { days }=11 / 25 \\
\text { total }(44 \%)\end{array}$ \\
\hline \multirow{2}{*}{$\begin{array}{c}>23 \text { and } \leq 32 \text { years of } \\
\text { age }\end{array}$} & $37 / 83(44.6 \%)$ & $14 / 30(46.66 \%)$ & $23 / 53(43.4 \%)$ \\
\hline & $\begin{array}{c}6>30 \text { days }=43 / 83 \\
(51.81 \%)\end{array}$ & $\begin{array}{c}2>30 \text { days }=16 / 30 \\
(53.33 \%)\end{array}$ & $\begin{array}{c}4>30 \text { days }=27 / 53 \\
(51 \%)\end{array}$ \\
\hline \multirow[b]{2}{*}{$33-43$ years of age } & $29 / 75(38.66 \%)$ & $4 / 18(22.22 \%)$ & $25 / 57(43.86 \%)$ \\
\hline & $\begin{array}{c}2>30 \text { days }=31 / 75 \\
(41.33 \%)\end{array}$ & $\begin{array}{c}0>30 \text { days }=4 / 18 \\
(22.22 \%)\end{array}$ & $\begin{array}{c}2>30 \text { days }=27 / 57 \\
(47.4 \%)\end{array}$ \\
\hline$\geq 44$ years of age & $19 / 41(46.34 \%)$ & $7 / 16(43.8 \%)$ & $12 / 25(48 \%)$ \\
\hline
\end{tabular}

Note: Table 4.4 shows linkage to care by age group \& compares the Standard of Care to the combined Experimental group ( $N=238)$.

Because literature on linkage to care shows rates vary for different age groups, analyses were run to inspect variations in linkage rates among different age groups. Chi square tests were conducted to compare differences between the combined Experimental group and the Standard of Care among the different 
age groups for linkage to care within 30 days and by post-test. For those aged 18-23, link within 30 days, Pearson's Chi square showed there was no significant difference $\left(X^{2}{ }_{(1)}=.043, p=.835\right)$, and for the same age group (18-23) link by posttest, Pearson's Chi square also found no significant difference $\left(X^{2}(1)=.255\right.$, $p=.614$ ). For those aged 24-32, link within 30 days, Pearson's Chi square found no significant difference $\left(X^{2}{ }_{(1)}=.083, p=.773\right)$, and for the same age group (2432) link by post-test, Pearson's Chi square found no significant difference $\left(X^{2}{ }_{(1)}=\right.$ $.044, p=.834)$. For those aged 33-43, link within 30 days, Pearson's Chi square found no significant difference $\left(X^{2}(1)=2.70, p=.100\right)$, and for the same age group (33-43), link by post-test, Pearson's Chi square showed the difference was trending towards significant $\left(X_{(1)}^{2}=3.57, p=.059\right)$. For those aged $44+$, link within 30 days, Pearson's Chi square showed there was no significant difference $\left(X_{(1)}^{2}=\right.$ $.007, p=.935)$, and for those of the same age group (44+) link by post-test, Pearson's Chi square showed there was no significant difference $\left(X_{(1)}^{2}=.071\right.$, $p=.790)$.

\subsection{Secondary Research Question 3A}

What is the difference in participant satisfaction scores between the combined Experimental groups and the Standard of Care group?

Hypothesis: Participants in the combined Experimental group (receiving any level of the intervention) will have higher satisfaction scores as compared to participants receiving only the Standard of Care.

Table 4.5 Satisfaction Responses I 


\begin{tabular}{cccc}
\hline Group & $\begin{array}{c}\text { Overall, the } \\
\text { information you } \\
\text { received was } \\
\text { helpful? }\end{array}$ & $\begin{array}{c}\text { It was helpful to be } \\
\text { asked about } \\
\text { difficulties in your } \\
\text { relationship? }\end{array}$ & $\begin{array}{c}\text { You regret talking } \\
\text { about the difficulties in } \\
\text { your relationship? }\end{array}$ \\
\hline $\begin{array}{c}\text { Standard of Care } \\
(79)\end{array}$ & $\begin{array}{c}\text { Yes=73 } \\
(92.41 \%)\end{array}$ & $\begin{array}{c}\text { Yes=76 } \\
(96.2 \%)\end{array}$ & $\begin{array}{c}\text { No=76 } \\
(96.2 \%)\end{array}$ \\
\hline $\begin{array}{c}\text { Combined Exp. } \\
\text { Group } \\
(160)\end{array}$ & $\begin{array}{c}\text { Yes=148 } \\
(92.5 \%)\end{array}$ & $\begin{array}{c}\text { Yes=156 } \\
(97.5 \%)\end{array}$ & $\begin{array}{c}\text { No=152 } \\
(95 \%)\end{array}$ \\
\hline $\begin{array}{c}\text { Total } \\
(239)\end{array}$ & $\begin{array}{c}\text { Yes, information } \\
\text { was helpful=221 } \\
(92.47 \%)\end{array}$ & $\begin{array}{c}\text { Yes, it was helpful to } \\
\text { be asked about } \\
\text { relationship=232 } \\
(97.07 \%)\end{array}$ & $\begin{array}{c}\text { Do not regret talking } \\
\text { about relationship= } \\
228 \\
(95.4 \%)\end{array}$
\end{tabular}

Note: Table 4.5 shows satisfaction responses between the Standard of Care group and the combined Experimental group $(N=239)$.

Most individuals (about 93\%) reported that the overall services they received were helpful. Responses were almost equal for the two groups, and a Pearson's Chi square test showed the difference was non-significant $\left(X_{(1)}^{2}=\right.$ $.001, p=.979)$.

The vast majority of people (about $97 \%$ ) reported it was helpful to be asked about the difficulties in their relationship. A higher percentage of people in the combined Experimental group reported it was helpful, but Fisher's Exact test showed the difference was non-significant $\left(X^{2}(1)=.313, p=.576\right)$.

The vast majority of people (almost $96 \%$ ) in both groups reported they did not regret talking about the difficulties in their relationship, and a Fisher's Exact test showed the difference between the two groups was non-significant $\left(X^{2}{ }_{(1)}=\right.$ $.174, p=1.00)$.

\subsection{Secondary Research Question 3B}


What is the difference in participant satisfaction, feasibility and acceptability scores between dosage levels of those who receive 1. danger indicator + initial safety plan, and 2 . danger indicator + initial safety plan + follow-up safety plan? Hypothesis: The possible impact of higher dosage intervention on satisfaction, acceptability and feasibility is uncertain.

\section{Satisfaction}

Table 4.6 Satisfaction Responses II

\begin{tabular}{cccc}
\hline Group & $\begin{array}{c}\text { Overall, the } \\
\text { information you } \\
\text { received was } \\
\text { helpful? }\end{array}$ & $\begin{array}{c}\text { It was helpful to be } \\
\text { asked about } \\
\text { difficulties in your } \\
\text { relationship? }\end{array}$ & $\begin{array}{c}\text { You regret talking } \\
\text { about the difficulties } \\
\text { in your relationship? }\end{array}$ \\
\hline Dosage level 1 (81) & Yes=74 (91.4\%) & Yes=79 (97.5\%) & No=76 (93.8\%) \\
\hline Dosage level 2 (79) & Yes=74 (93.7\%) & Yes=77 (97.5\%) & No=76 (96.2\%) \\
\hline
\end{tabular}

Note: Table 4.6 shows satisfaction responses between Dosage Levels $(N=160)$.

The majority of participants found the information they received to be helpful, but more participants reported the information was helpful in Dosage level two group. However, a Chi square test showed the difference was nonsignificant $\left(X_{(1)}^{2}=.308, p=.579\right)$.

The vast majority of participants from both groups reported that it was helpful to be asked about the difficulties in their relationship, with an equal amount giving this positive rating from each group; thus, Fisher's exact Test found the difference was non-significant $\left(X_{(1)}^{2}=.001, p=1.00\right)$. 
While most participants from both groups reported they did not regret talking about the difficulties in their relationship, more individuals in Dosage level two reported they had less regret. Fisher's Exact Test showed the difference was non-significant $\left(X_{(1)}^{2}=.475, p=.720\right)$.

\section{Acceptability}

Table 4.7 Acceptability Responses

\begin{tabular}{|c|c|c|c|}
\hline Group & $\begin{array}{l}\text { Was safety plan } \\
\text { helpful? }\end{array}$ & $\begin{array}{l}\text { Would you } \\
\text { recommend it for a } \\
\text { friend? }\end{array}$ & $\begin{array}{c}\text { Did you use any of } \\
\text { the safety } \\
\text { strategies? }\end{array}$ \\
\hline $\begin{array}{c}\text { Dosage level } 1 \\
\text { (81) }\end{array}$ & $\begin{array}{c}\text { Yes }=64 \\
(79.1 \%) \\
\text { No=17 } \\
(20.9 \%)\end{array}$ & $\begin{array}{c}\text { Yes }=77 \\
(95 \%) \\
\text { No= } 4 \\
(4.9 \%)\end{array}$ & $\begin{array}{c}\mathrm{No}=64 \\
(79.01 \%) \\
\mathrm{No}=17 \\
(20.98 \%)\end{array}$ \\
\hline $\begin{array}{c}\text { Dosage level } 2 \\
\text { (79) }\end{array}$ & $\begin{array}{c}\text { Yes=64 } \\
(81 \%) \\
\text { No=15 } \\
(19 \%)\end{array}$ & $\begin{array}{c}\text { Yes }=79 \\
(100 \%) \\
\text { No=0 } \\
(0 \%)\end{array}$ & $\begin{array}{l}\text { Yes }=65 \\
(82.3 \%) \\
\text { No= } 14 \\
(17.7 \%)\end{array}$ \\
\hline
\end{tabular}

Note: Table 4.7 shows acceptability responses between Dosage Levels $(N=160)$.

There was a slightly higher percentage of people in the Dosage level 2 group who reported the safety plan was helpful, but Pearson's Chi square showed the difference was non-significant $\left(X^{2}{ }_{(1)}=.100, p=.752\right)$. A higher percentage of those in Dosage level 2 reported they would recommend the safety plan for a friend, but Fisher's exact test showed the difference was nonsignificant $\left(X^{2}{ }_{(1)}=4.00, p=.120\right)$. A higher percentage of those in Dosage level 2 also reported having used at least one safety strategy, but Fisher's exact test showed the difference was non-significant $\left(X^{2}{ }_{(1)}=.273, p=.690\right)$. 
For the combined experimental group, of those who used at least one safety strategy, $114 / 129$ found the safety plan overall helpful $(88.4 \%)$, and $28 / 31$ $(90.32 \%)$ who reported having not used any safety strategies, still found the safety plan overall helpful. Chi square showed the difference of the use of the safety plan between those who used strategies and did not use strategies was significant $\left(X^{2}(1)=29.17, p=.000\right)$. Fisher's Exact Test showed that significantly more people who used at least one safety strategy found the safety plan* to be helpful than those who did not use a safety strategy $\left(X^{2}{ }_{(1)}=8.13, p=.023\right)$.

Of the 129 who used at least one safety strategy, $127 / 129(98.5 \%)$ would recommend it for a friend. Of those who used at least one safety strategy, $126 / 129(97.7 \%)$ reported no violence was experienced if they notified their partner of their serostatus. Of those using a safety strategy who did experience violence upon partner notification of serostatus, 2 were in Dosage level 1 group, and 1 was in Dosage level 2 group. Of those employing 2 or more safety strategies, $127 / 129(98.5 \%)$ reported no violence experienced if they notified their partner of their serostatus. Of those 2 reporting violence, both were in Dosage level 1 , and 0 were in Dosage level 2. Of those who used 3 or more strategies, only 1 person reported violence as a result of serostatus notification, and this respondent reported experiencing violence despite having used 7 safety strategies. Hence, this suggests that with the use of each additional safety strategy, coupled with the extra dosage level, participants fair better upon partner notification (experienced less violence as a result). 
For all participants, it was trending towards significant that for those who used at least one safety strategy more linked to care by post-test, one-sided $\left(X^{2}{ }_{(1)}\right.$ $=2.46, p=.085)$. Of those who employed at least one safety strategy, it was trending towards significant that more people linked to care (by post-test) in Dosage level 1 (38/64) (59.4\%) compared to Dosage level 2 (28/65) (43.1\%) $\left(X^{2}(1)=3.43, p=.064\right)$. Of those who employed 2 or more safety strategies, significantly more people linked to care (by post-test) in Dosage level $1(25 / 40)$ $(62.5 \%)$ compared to Dosage level $2(16 / 42)(38.1 \%)\left(X^{2}{ }_{(1)}=4.88, p=.046\right)$. This suggests that with the use of any safety strategy coupled with a higher dosage level of safety planning, linkage to care may be delayed.

\section{Feasibility}

Table 4.8 Feasibility Responses

\begin{tabular}{|c|c|c|c|c|}
\hline Group & $\begin{array}{l}\text { Domain 1: } \\
\text { Time to } \\
\text { Reach }\end{array}$ & $\begin{array}{l}\text { Domain 2: } \\
\text { Length of } \\
\text { Intervention }\end{array}$ & $\begin{array}{c}\text { Domain 3: } \\
\text { Techniques } \\
\text { used }\end{array}$ & Domain 4: Risk \\
\hline $\begin{array}{c}\text { Dosage } \\
\text { level } 1 \\
\text { (81) }\end{array}$ & $\begin{array}{l}\text { Pre-test: } \\
3.7 \text { times } \\
\text { Post-test: } \\
5.95 \text { times }\end{array}$ & $\begin{array}{l}\text { Pre-test: } \\
9.62 \text { mins } \\
\text { Post-test: } \\
6.52 \text { mins } \\
\text { Initial SP: } \\
8.62 \text { mins }\end{array}$ & $\begin{array}{l}\text { Use of } \\
\text { Safety plan } \\
49 / 81 \\
(60.5 \%) \\
1.76 \\
\text { strategies } \\
\text { used }\end{array}$ & $\begin{array}{c}\text { Violence upon partner notification: } \\
\text { No= } 77 / 81(95.1 \%) \\
\text { Safe to contact again: } \\
\text { Yes }=78 / 81(96.3 \%) \\
\text { Talking placed you in more danger: } \\
\text { No= } 78 / 81(96.3 \%) \\
\text { Services placed you in more danger: } \\
\text { No }=77 / 81(95.1 \%)\end{array}$ \\
\hline $\begin{array}{c}\text { Dosage } \\
\text { level } 2 \\
\text { (79) }\end{array}$ & $\begin{array}{c}\text { Pre-test: } \\
4.2 \text { times } \\
\text { Post-test: } \\
6.57 \text { times } \\
\text { Follow-up } \\
\text { safety } \\
\text { plan: } \\
13.05 \\
\text { times }\end{array}$ & $\begin{array}{l}\text { Pre-test: } \\
10.56 \text { mins } \\
\text { Post-test: } \\
6.56 \text { mins } \\
\text { Initial } \\
\text { Safety plan: } \\
9.68 \text { mins } \\
\text { Follow-up } \\
\text { safety plan: } \\
4.81 \text { mins }\end{array}$ & $\begin{array}{l}\text { Use of } \\
\text { Safety plan } \\
50 / 79 \\
(63.3 \%) \\
\\
2.18 \\
\text { strategies } \\
\text { used }\end{array}$ & $\begin{array}{c}\text { Violence upon partner notification: } \\
\text { No }=78 / 79(98.7 \%) \\
\text { Safe to contact again: } \\
\text { Yes }=76 / 79(96.2 \%) \\
\text { Talking placed you in more danger: } \\
\text { No }=79 / 79(100 \%) \\
\text { Services placed you in more danger: } \\
\text { No }=77 / 79(97.5 \%)\end{array}$ \\
\hline
\end{tabular}


Domain 1: Time to Reach

When comparing attempts to reach for post-test only, it took slightly fewer attempts to reach those in Dosage level 1 compared to Dosage level 2, but an Independent sample t-test showed the difference was non-significant $t(1(158)$ ($.473), p=.637)$.

Domain 2: Time to administer

It took about 2 minutes longer to administer the intervention to those in Dosage Level 2, excluding the follow-up safety plan, compared to Dosage Level One $(D 1=M=24.75, S D=6.67, S E M=.74 ; D 2=M=26.81, S D=7.01, S E M=.79)$. With the follow-up safety plan included, Dosage level 2 had a mean of 31.51 minutes $(M=31.51, S D=7.63, S E M=.87)$, and the follow-up safety plan alone took a mean of 4.81 minutes $(M=4.81, S D=2.02, S E M=.229)$.

\section{Domain 3: Use of safety techniques}

A similar number of participants in Dosage level 1 and Dosage level 2 reported having used the safety plan (see table 4.8), and Pearson's Chi square test revealed the difference was non-significant $\left(X_{(1)}^{2}=.100, p=.844\right)$.

Participants in Dosage level 2 reported using more safety strategies than those in Dosage level 1 (see table 4.8), but an Independent sample t-test showed the difference was non-significant $(t(1(158)(-1.04), p=.300)$. However, a higher percentage of those in Dosage level 2 group used slightly more strategies than 
those in dosage level 1 group (D1: $M=1.88, S D=1.76, S E M=.20$; $D 2: M=2.18$, $S D=1.9, S E M=.21$ ), suggesting that the extra dosage level (follow-up safety plan) could have contributed to the increased use of strategies.

The use of any of the consultation strategy was merged into one Consultation category. Of all the consultation categories (consulted with medical professional, counselor, clergy, IPV provider, family or friend, or legal), 128 (80\%) participants from the combined Experimental group reported having used a consultation strategy. The amount of people who used any consultation strategy was significantly higher among the combined Experimental group (80\%) compared to the Standard of Care group $(4 \%)\left(X^{2}{ }_{(1)}=127.26, p=.000\right)$. Additionally, a higher percentage of people in Dosage level 2 group (65/79 or $82.28 \%$ ) used at least one consultation strategy than those in Dosage level 1 group $(63 / 77$ or $77.78 \%)$.

Figure 4.5 Number of Safety Strategies Used

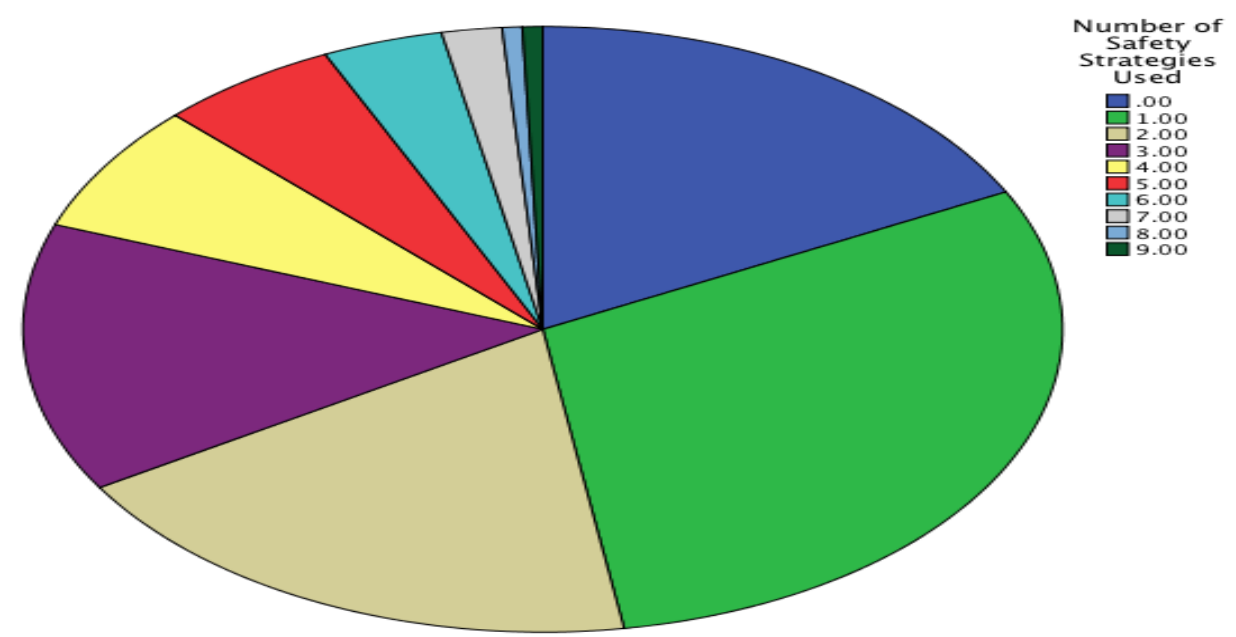

Figure 4.5 shows the number of safety strategies used by those in the combined Experimental group $(N=160)$. 
About $19 \%$ of the participants reported using no safety strategies at all (31/160), and eight people (5\%) reported using the Fighting Back technique which was counter to that recommended in the safety plan (safety plan informed participants that fighting back techniques have been shown to increase violence). The complexity of this strategy will be discussed in Chapter Five, as the item included several strategies within one per domain categorization by Parker \& Gielen (2014). 1/8 of those who used this strategy only used this strategy alone, and this was coded as a "no" for use of any strategy. Most of the participants who used a safety plan used only 1 strategy (29.4\%). However, $19.4 \%$ used 2 strategies, $14.4 \%$ used 3 strategies, $5.6 \%$ used 4 strategies, $5.6 \%$ used 5 strategies, $3 \%$ used 6 strategies, $2.5 \%$ used 7 strategies, and .6\% used 8 strategies.

Figure 4.6 Frequency of Safety Strategies Used

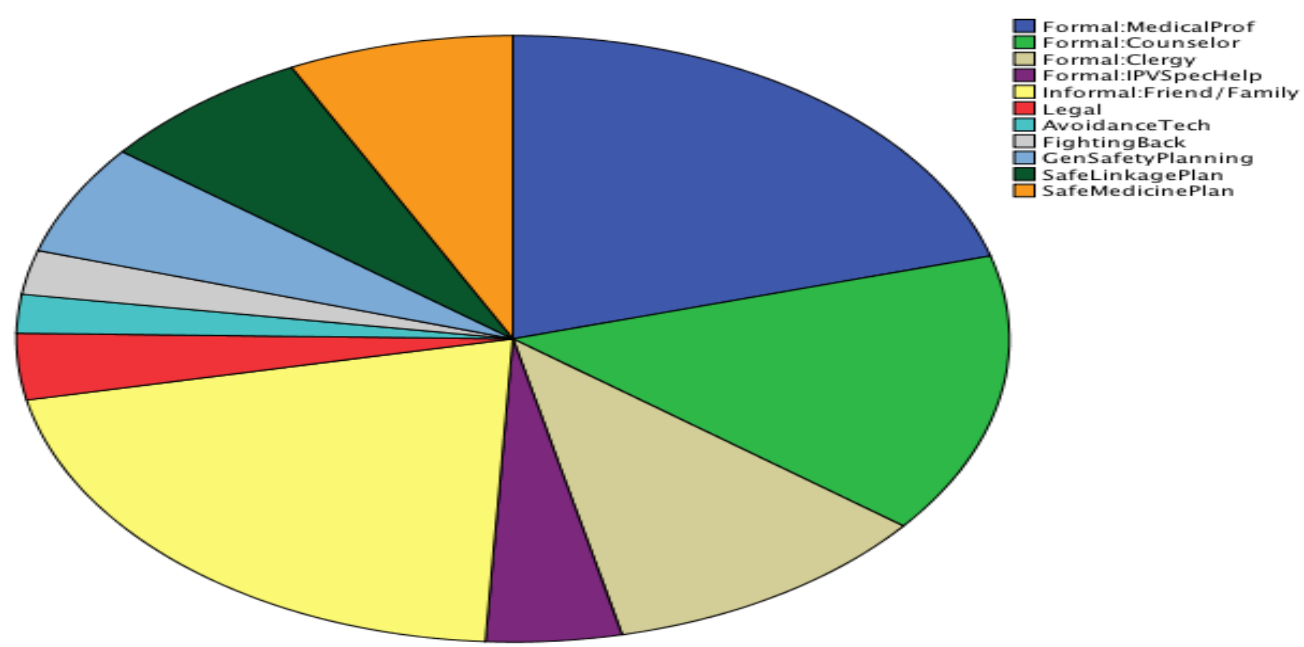

Figure 4.6 shows the frequency of safety strategies used, by those who used any strategy at all, within the combined Experimental group $(N=130)$. 
Table 4.9 Frequency of Safety Strategies Used

\begin{tabular}{|c|c|c|c|c|}
\hline Strategy & Use: No & Use: Yes & $\begin{array}{c}\% \text { Who } \\
\text { used } \\
\text { Strategy \& } \\
\text { Linked to } \\
\text { Care (\% out } \\
\text { of those who } \\
\text { used strategy) }\end{array}$ & $\begin{array}{l}\% \text { Who Did not } \\
\text { use strategy but } \\
\text { linked to care (\% } \\
\text { out of those who did } \\
\text { not use the strategy) }\end{array}$ \\
\hline Formal: Medical Professional & $\begin{array}{c}90 \\
(56 \%)\end{array}$ & $\begin{array}{c}70 \\
(44 \%)\end{array}$ & $\begin{array}{c}33 \\
(47.14 \%)\end{array}$ & $\begin{array}{c}44 / 90 \\
(48.89 \%)\end{array}$ \\
\hline Formal: Counselor & $\begin{array}{c}109 \\
(68 \%) \\
\end{array}$ & $\begin{array}{c}51 \\
(32 \%) \\
\end{array}$ & $\begin{array}{c}14 \\
(27.5 \%) \\
\end{array}$ & $\begin{array}{l}63 / 109 \\
(57.8 \%) \\
\end{array}$ \\
\hline Formal: Clergy & $\begin{array}{c}123 \\
(77 \%) \\
\end{array}$ & $\begin{array}{c}37 \\
(23 \%) \\
\end{array}$ & $\begin{array}{c}13 \\
(35.14 \%) \\
\end{array}$ & $\begin{array}{c}64 / 123 \\
(52.03 \%) \\
\end{array}$ \\
\hline Formal: IPV specific service & $\begin{array}{c}145 \\
(91 \%)\end{array}$ & $\begin{array}{l}15 \\
(9 \%)\end{array}$ & $\begin{array}{c}5 \\
(33.33 \%)\end{array}$ & $\begin{array}{c}72 / 145 \\
(49.66 \%)\end{array}$ \\
\hline Informal: Family or friend & $\begin{array}{c}89 \\
(56 \%)\end{array}$ & $\begin{array}{c}71 \\
(44 \%)\end{array}$ & $\begin{array}{c}43 \\
(60.6 \%)\end{array}$ & $\begin{array}{c}34 / 89 \\
(38.20 \%)\end{array}$ \\
\hline Legal & $\begin{array}{c}148 \\
(92.5 \%) \\
\end{array}$ & $\begin{array}{c}12 \\
(7.5 \%)\end{array}$ & $\begin{array}{c}4 \\
(33.33 \%) \\
\end{array}$ & $\begin{array}{c}73 / 148 \\
(49.32 \%)\end{array}$ \\
\hline $\begin{array}{c}\text { Avoidance/Pacification } \\
\text { technique }\end{array}$ & $\begin{array}{c}153 \\
(96 \%) \\
\end{array}$ & $\begin{array}{c}7 \\
(4 \%)\end{array}$ & $\begin{array}{c}5 \\
(71.4 \%) \\
\end{array}$ & $\begin{array}{c}72 / 153 \\
(47.06 \%)\end{array}$ \\
\hline Fighting back (subtract point ) & $\begin{array}{c}152 \\
(95 \%) \\
\end{array}$ & $\begin{array}{c}8 \\
(5 \%) \\
\end{array}$ & $\begin{array}{c}2 \\
(2.5 \%) \\
\end{array}$ & $\begin{array}{c}75 / 152 \\
(49.34 \%)\end{array}$ \\
\hline $\begin{array}{c}\text { General Safety planning } \\
\text { technique }\end{array}$ & $\begin{array}{c}140 \\
(87.5 \%) \\
\end{array}$ & $\begin{array}{c}20 \\
(12.5 \%) \\
\end{array}$ & $\begin{array}{c}8 \\
(40 \%) \\
\end{array}$ & $\begin{array}{c}69 / 140 \\
(49.29 \%) \\
\end{array}$ \\
\hline $\begin{array}{l}\text { Plan for Safe linkage to } \\
\text { medical care }\end{array}$ & $\begin{array}{c}136 \\
(85 \%)\end{array}$ & $\begin{array}{c}24 \\
(15 \%)\end{array}$ & $\begin{array}{c}10 \\
(41.67 \%)\end{array}$ & $\begin{array}{c}67 / 136 \\
(49.26 \%)\end{array}$ \\
\hline $\begin{array}{l}\text { Plan for safely taking } \\
\text { medicine }\end{array}$ & $\begin{array}{c}135 \\
(84 \%)\end{array}$ & $\begin{array}{c}25 \\
(16 \%)\end{array}$ & $\begin{array}{c}8 \\
(32 \%)\end{array}$ & $\begin{array}{c}69 / 135 \\
(51.11 \%)\end{array}$ \\
\hline
\end{tabular}

Note: Table 4.9 shows the frequency of safety strategies used by those in the combined Experimental group and the percentage of individuals within the combined Experimental group who used a strategy and linked to care as well as those who did not use a strategy but still linked to care $(N=160)$.

As noted above, a trend was observed that more participants using at least one safety strategy linked to care. The amount of people in the combined Experimental group who used a consultation strategy and linked to care $(66 / 77$ or $85.71 \%$ ) was higher than those who linked to care but did not use a consultation strategy (11/77 or $14.28 \%)$. More people who used any consultation strategy also linked to care $(66 / 128$ or $51.56 \%))$ compared to those who used a consultation strategy but did not link to care $(62 / 128$ or $48.44 \%)$. The difference was trending towards significant $\left(X_{(1)}^{2}=3.03, p=.082\right)$. 
For the combined Experimental group, more participants who used any consultation strategy linked to care by post-test than those in the Standard of Care group. The difference was trending towards significant $\left(X^{2}{ }_{(1)}=2.57\right.$, $p=.072)$.

For the combined Experimental group, more participants who used any consultation strategy reported less violence as a result if they notified their partner of their serostatus compared to the Standard of Care group. A Chi square test showed the difference was significant $\left(X^{2}{ }_{(1)}=8.51, p=.005\right)$.

A Pearson's Chi square showed that those who talked with a friend/family member were significantly more likely to link to care than those who did not use the strategy $\left(X^{2}(1)=7.91 p=.005\right)$.

\section{Domain 4: Risk level}

For perceived safety to be contacted again (in one month, two months or three months, any of the above equaling yes), a Fisher's exact test showed the difference between dosage levels was non-significant $\left(X^{2}{ }_{(1)}=.001 p=1.00\right)$.

For perception that talking about the relationship difficulties placed one at greater danger, a Fisher's exact test showed the difference between dosage levels was non-significant $\left(X^{2}{ }_{(1)}=2.98 p=.245\right)$.

For perception that the services received placed one at greater danger, a Chi square test using Fisher's Exact test showed that the difference between dosage levels was non-significant $\left(X^{2}{ }_{(1)}=.642 p=.682\right)$. 
For violence experienced as a result of serostatus notification, a chi square test showed the difference between dosage levels was non-significant $\left(X^{2}{ }_{(1)}=1.77 p=.183\right)$, but Pearson's chi square showed the difference between the combined Experimental group (5/160 (3.13\%)) and the Standard of Care group $(10 / 81(12.35 \%))$ was significant $\left(X^{2}{ }_{(1)}=7.83, p=.005\right)$. Although the difference between dosage levels was non-significant, the amount of violence reported as a result of serostatus notification was attenuated with more Dosage level received as those in dosage level one had $4 / 81$ (4.9\%) report violence as a result of notification, and those in Dosage level 2 had a lower percentage with only $1 / 79(1.3 \%)$ reporting violence as a result of notification. Only $1 / 8$ people who used the fighting back strategy experienced violence as a result of serostatus notification.

\subsection{Additional Analyses}

\section{Forms of IPV}

Table 4.10 Forms of IPV

\begin{tabular}{ccccc}
\hline Group & $\begin{array}{c}\text { Non-violent } \\
\text { control }\end{array}$ & $\begin{array}{c}\text { Physical } \\
\text { abuse }\end{array}$ & $\begin{array}{c}\text { Sexual } \\
\text { abuse }\end{array}$ & $\begin{array}{c}\text { Physical or } \\
\text { Sexual abuse } \\
\text { only }\end{array}$ \\
\hline $\begin{array}{c}\text { Standard of Care } \\
(82)\end{array}$ & $\begin{array}{c}82 / 82 \\
(100 \%)\end{array}$ & $\begin{array}{c}26 / 82 \\
(31.71 \%)\end{array}$ & $\begin{array}{c}35 / 82 \\
(42.68 \%)\end{array}$ & $\begin{array}{c}48 / 82 \\
(58.53 \%)\end{array}$ \\
\hline $\begin{array}{c}\text { Combined } \\
\text { Experimental }\end{array}$ & $164 / 166$ & $75 / 166$ & $81 / 166$ & $119 / 166$ \\
group (166) & $(98.80 \%)$ & $(45.18 \%)$ & $(48.8 \%)$ & $(71.69 \%)$ \\
\hline Dosage level 1 & $81 / 83$ & $42 / 83$ & $39 / 83$ & $62 / 83$ \\
(83) & $(97.60 \%)$ & $(50.60 \%)$ & $(46.99 \%)$ & $(74.7 \%)$ \\
\hline Dosage level 2 & $83 / 83$ & $33 / 83$ & $42 / 83$ & $57 / 83$ \\
(83) & $(100 \%)$ & $(39.76 \%)$ & $(51 \%)$ & $(68.67)$
\end{tabular}

Note: Table 4.10 shows the forms of IPV reported by those in the Standard of Care and combined Experimental group and also provides a break down of IPV reported among each 
dosage level group $(N=248)$. The physical or sexual category simply considers how participants reported having experienced one of the two forms.

\section{Non-violent Control}

Figure 4.7 Non-violent Control

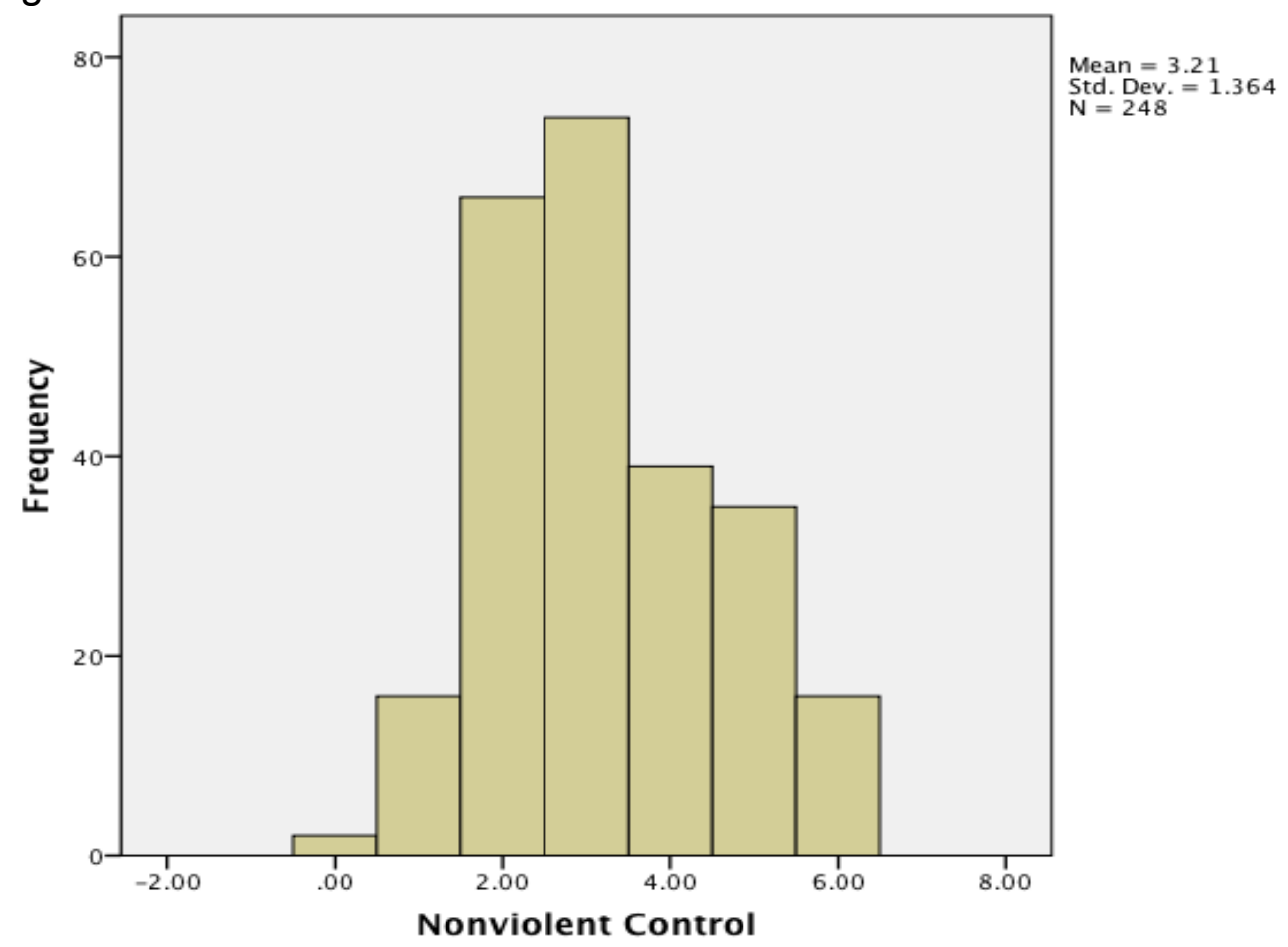

Figure 4.7 shows the amount of non-violent control tactics all participants endorsed having experienced from a scale of $0-6$ tactics $(N=248)$.

All participants but two reported having experienced some form of nonviolent control in their current relationship. $6.5 \%$ answered affirmative to only one item; $26.6 \%$ answered affirmative to two items; $29.8 \%$ answered affirmative to three items; $15.7 \%$ answered affirmative to four items; $14.1 \%$ answered affirmative to five items; and $6.5 \%$ answered affirmative to all six non-violent control items. 
The number of participants who reported having experienced at least one form of non-violent control was similar to those in the Standard of Care group and the combined Experimental group, and Fisher's exact test showed the difference was non-significant $\left(X_{(1)}^{2}=.996, p=1.00\right)$.

Of those who answered no to Your current partner insists on knowing where you are all the time, significantly more linked to care in the Experimental group (19/29 or $65.52 \%)$ than the Standard of Care group (3/15 or $20 \%)$. Chi square showed the difference was significant $\left(X^{2}{ }_{(1)}=8.19, p=.004\right)$.

Physical Abuse

Figure 4.8 Physical Abuse

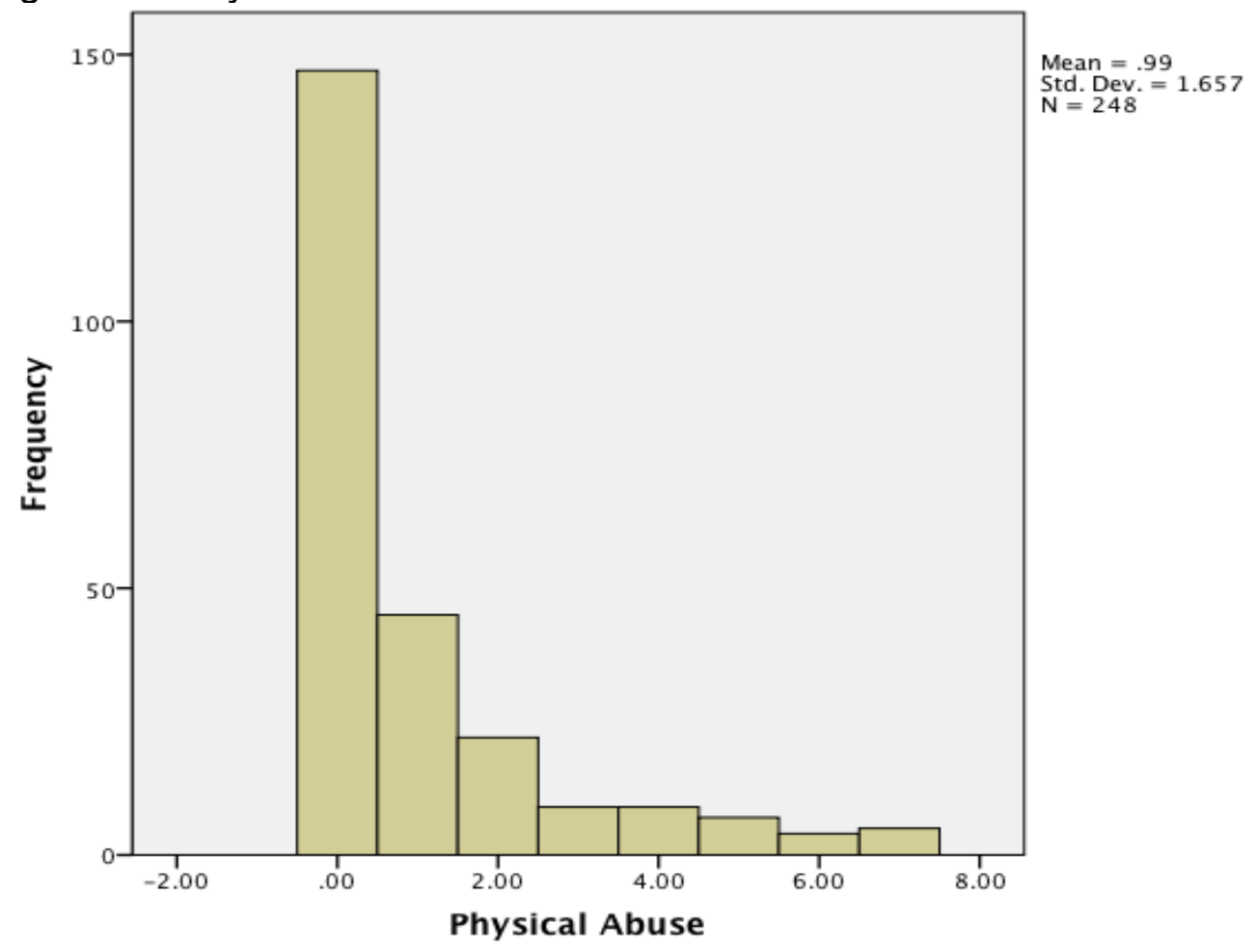

Figure 4.8 shows the amount of physical abuse tactics all participants endorsed having experienced from a scale of 0-7 $(N=248)$. 
$150(60.5 \%)$ reported no physical abuse, but the remaining 98 (39.5\%) answered affirmative to one or more items on physical abuse (in current relationship within the last year). $18.1 \%$ answered affirmative to 1 item; $8.9 \%$ answered affirmative to 2 items; $3.6 \%$ answered affirmative to 3 items; $3.6 \%$ answered affirmative to 4 items; $2.8 \%$ answered affirmative to 5 items; $1.6 \%$ answered affirmative to 6 items; $2 \%$ answered affirmative to all 7 physical abuse items.

Pearson's Chi square showed that significantly more people in the Experimental group reported experiencing at least one form of physical abuse at pre-test data collection than those in the Standard of Care group $\left(X^{2}{ }_{(1)}=4.13\right.$ $p=.042)$.

\section{Sexual Abuse}

Figure 4.9 Sexual Abuse

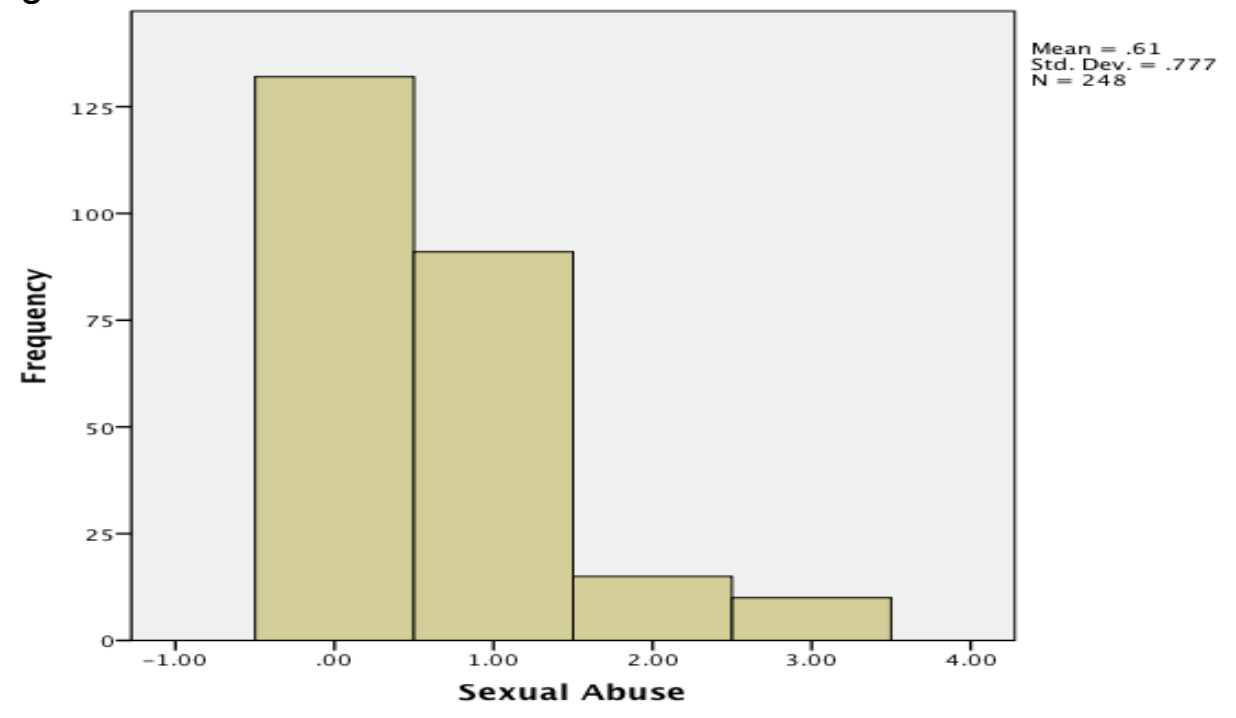


Figure 4.9 shows the amount of sexual abuse tactics all participants endorsed having experienced from a scale of 0-3 $(N=248)$.

$132(53.2 \%)$ participants reported having no sexual abuse in their relationship, but about $36 \%$ answered affirmative to at least one item on sexual abuse, and about $11 \%$ answered affirmative to at least 2 items on sexual abuse (in current relationship within the last year). The item with the highest percentage of affirmative answers was In the last year has your partner... "made you have sex without a condom," (110 or $44.4 \%$ ) followed by "used force to make you have sex" (30 or $12.1 \%$ ) and "used force to make you have anal or oral sex" (13 or $5.2 \%)$. While there were higher percentages of those in the combined Experimental group who reported having experienced at least one form of sexual abuse, Pearson's Chi square showed the difference was non-significant $\left(X_{(1)}^{2}=\right.$ $.824, p=.364)$.

Other sources reporting on IPV occurrence often use the inclusion of any physical or sexual assault in the last year, and therefore percentages of those with only physical or sexual assault were investigated. Those in the combined Experimental group (119/166 or $71.69 \%$ ) had significantly higher reports of having experienced physical or sexual assault in the last year compared to the Standard of Care group (48/82 or $58.53 \%)\left(X^{2}{ }_{(1)}=4.32, p=.038\right)$, but when the amount of assault experienced (number of tactics) was compared between the groups, although it remained higher for the combined Experimental group $(M=1.75 ; S D=2.12 ; S E M=.16)$ than the Standard of Care group $(M=1.33$; $S D=1.76$; $S E M=.19$ ), an Independent sample t test showed the difference was 
non-significant $(t(1,246)=-1.57 ; p=.270)$.

Figure 4.10 Danger Indicator Scores

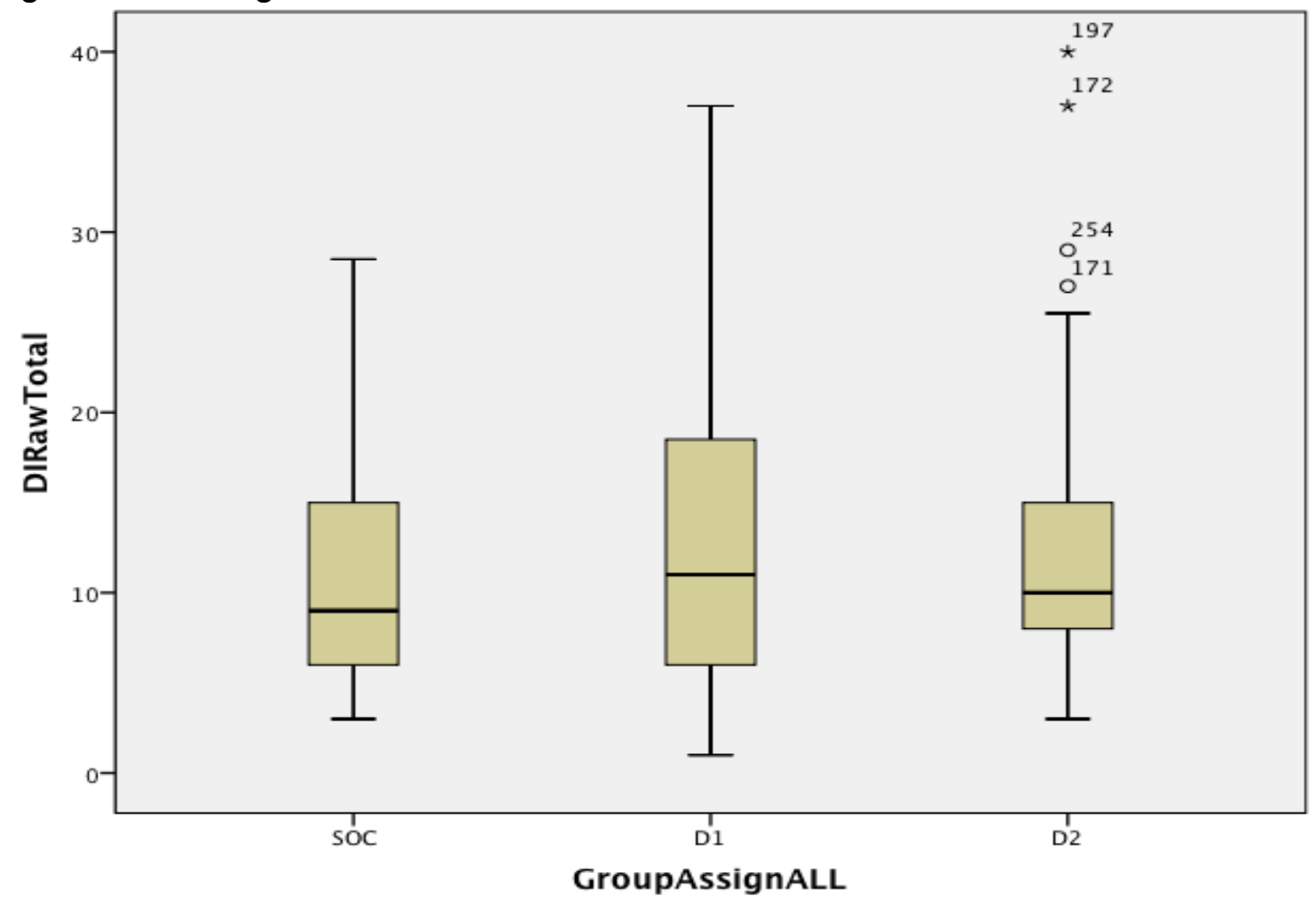

Figure 4.10 shows Danger Indicator scores for the Standard of Care group (SOC) and each dosage level (D1 and D2) $(N=248)$.

For Danger Indicator scores, Dosage Level 1 had the highest raw scores $(M=13.15, S D=8.65, S E M=.96)$ followed by Dosage Level $2(M=11.95, S D=$ 7.16, $S E M=.82)$ and then the Standard of Care group with the lowest scores $(M=$ $10.52, S D=6.06, S E M=.69$ ). These scores were out of a possible $0-49$ points.

Danger Levels, Re-assault \& Partner Notification

Table 4.11 Danger Score Comparisons 


\begin{tabular}{ccccc} 
Group & Pre HIRS score & $\begin{array}{c}\text { Post HIRS } \\
\text { score }\end{array}$ & DA score & $\begin{array}{c}\text { Danger } \\
\text { Indicator score }\end{array}$ \\
\hline SOC & $M=2.29$ & $M=2.17$ & $M=2.63$ & $M=10.52$ \\
& $S D=2.74$ & $S D=2.07$ & $S D=1.91$ & $S D=6.06$ \\
& $S E M=.31$ & $S E M=.23$ & $S E M=.22$ & $S E M=.69$ \\
\hline Combined Exp. & $M=2.51$ & $M=2.84$ & $M=2.88$ & $M=12.57$ \\
group & $S D=2.97$ & $S D=2.17$ & $S D=2.00$ & $S D=7.96$ \\
& $S E M=.24$ & $S E M=.17$ & $S E M=.16$ & $S E M=.63$ \\
\hline \multirow{2}{*}{ Dosage Level 1 } & $M=2.51$ & $M=2.84$ & $M=3.03$ & $M=13.15$ \\
& $S D=3.03$ & $S D=2.45$ & $S D=2.2$ & $S D=8.65$ \\
& $S E M=.34$ & $S E M=.25$ & $S E M=.24$ & $S E M=.96$ \\
Dosage Level 2 & $M=2.51$ & $M=2.84$ & $M=2.72$ & $M=11.95$ \\
& $S D=2.02$ & $S D=2.1$ & $S D=1.78$ & $S D=7.16$ \\
& $S E M=.33$ & $S E M=.24$ & $S E M=.20$ & $S E M=.82$
\end{tabular}

Note: Table 4.11 shows score comparisons between the Standard of Care and combined Experimental group as well as a break down of each dosage level in the experimental group for pre and post HIRS scores, Danger Assessment scores and Danger Indicator scores. It does not include those individuals who were considered contaminating cases in the Standard of Care group $(N=239)$.

The DA scores are included to demonstrate that the Combined Experimental group also had a slightly higher score on the DA, but an Independent sample t-test showed the difference was non-significant $(t(237)=$ $.916,(p=.361)$.

However, an independent sample t-test showed that those in the Standard of Care group scored significantly lower than the Combined Experimental group on the Danger Indicator at pre-test $(t(234)=-2.00,(p=.046)$. According to this, those in the experimental group would be predicted to have a slightly higher occurrence of re-assault.

When controlling for sexual abuse, it remained that those in the Standard of Care group had significantly more people experiencing re-assault, this was trending towards significant for both those who reported sexual abuse $\left(X^{2}(1)=\right.$ 
$4.18 p=.055$ ), but only trending towards significant (one-sided) for those who did not have sexual abuse and $\left(X^{2}{ }_{(1)}=3.9 p=.061\right)$.

However, all participant groups reported an increase in the questions You feel safe getting to medical appointments (pre) (236/248 or 95.16\%) and You have felt safe getting to medical appointments (post) (102/248 or $41.13 \%)$. A McNemar test showed that the pre-post difference for the total sample was significant $\left(X^{2}{ }_{(1)}=.000 p=.000\right)$. This indicates that significantly more participants experienced a decrease in their perceived safety in getting to medical appointments within the first 30 days after testing.

Figure 4.11 Pre-test Perceived Safety in Getting to Medical Appointments

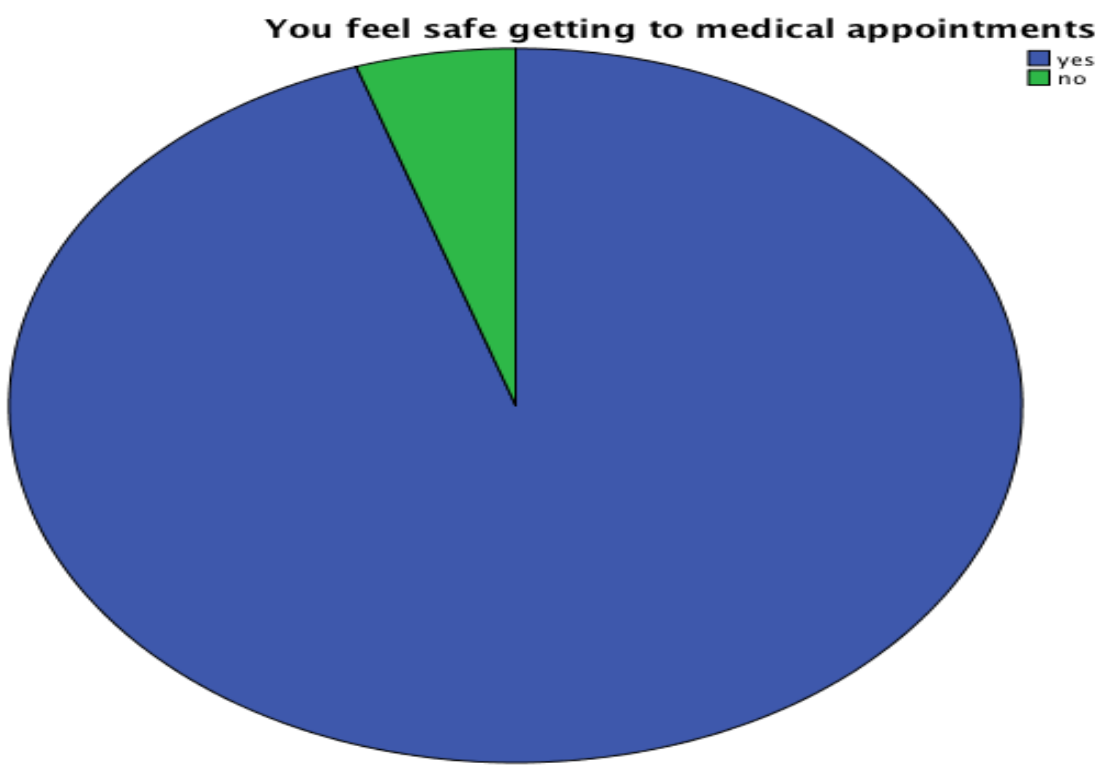

Figure 4.11 shows the amount of participants in the total sample who responded "Yes" to the item You feel safe getting to medical appointments during the pre-test $(N=248)$. 
Figure 4.12 Post-test Perceived Safety in Getting to Medical Appointments You have felt safe getting to medical appointments

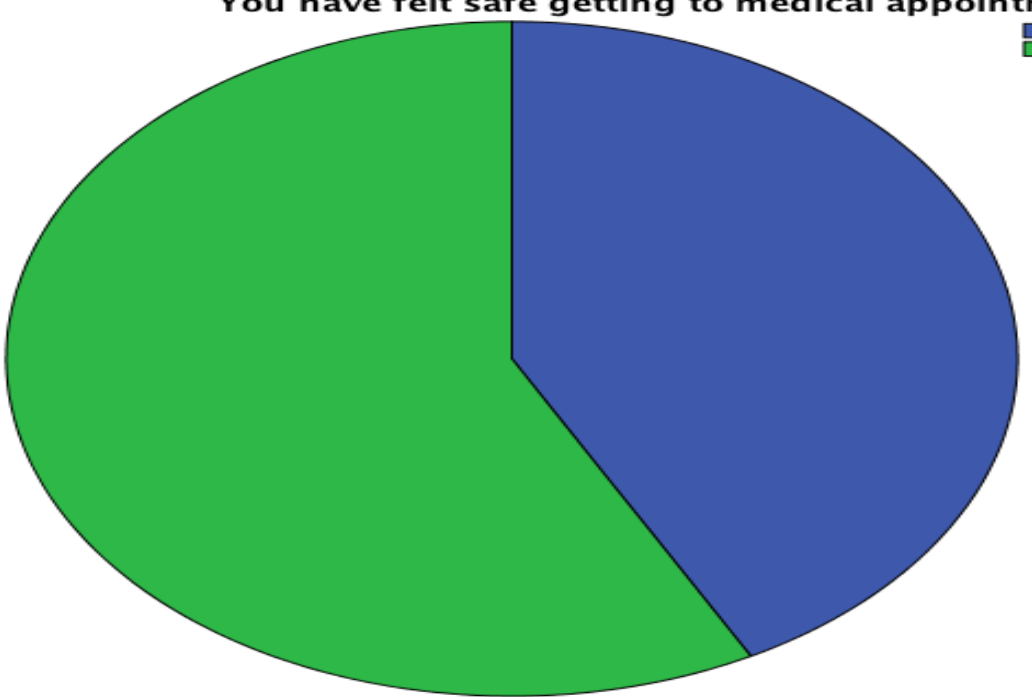

Figure 4.12 shows the amount of participants in the total sample who responded "Yes" to the item You have felt safe getting to medical appointments during the post-test $(N=248)$.

\section{Tester Status}

Figure 4.13 Tester Status Distribution

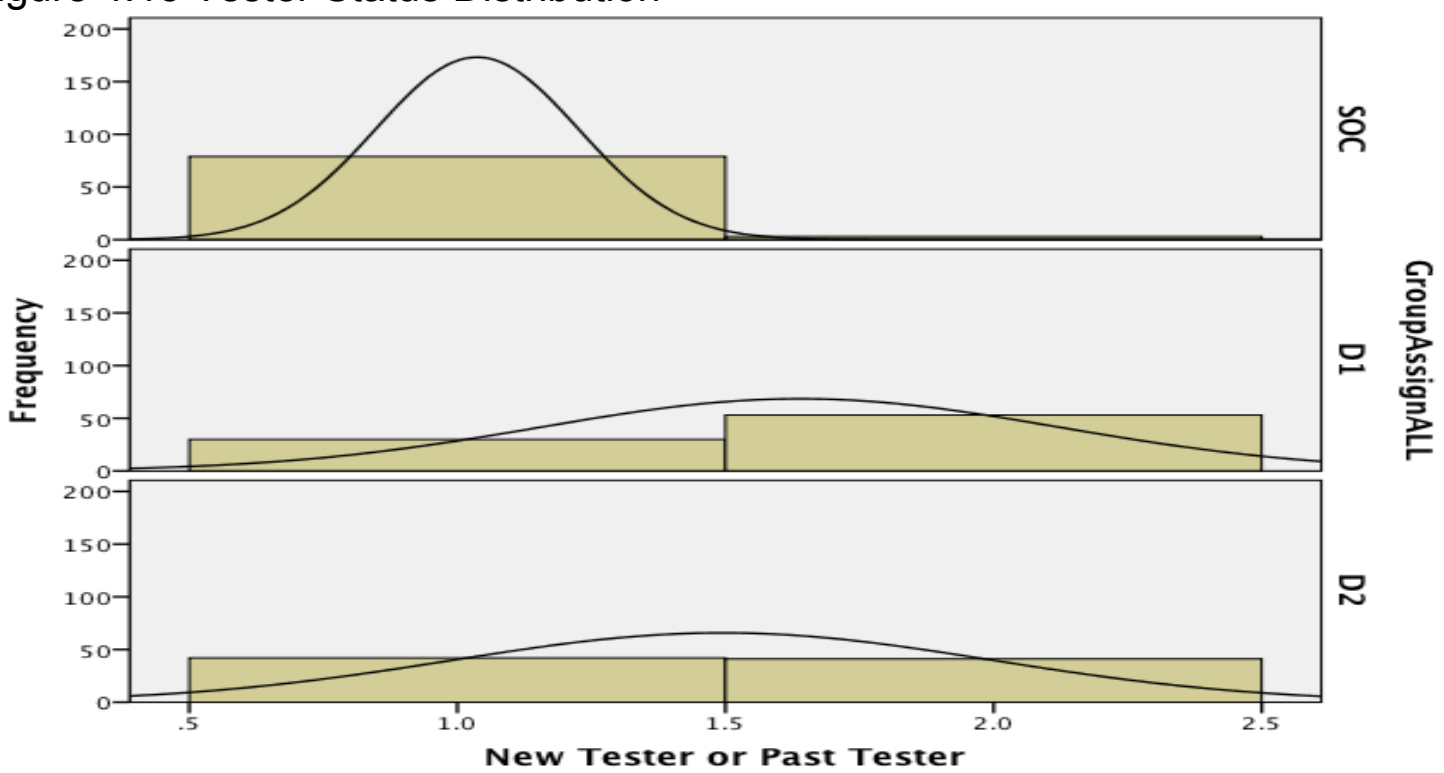

Figure 4.13 demonstrates the unequal distribution between study groups of those who had been previously diagnosed as HIV+. Those who had been previously diagnosed are listed as Past tester, and those who were newly diagnosed are listed as New tester $(N=248)$. 
Table 4.12 Tester Status

\begin{tabular}{ccc}
\hline Group & New Tester (1) & Past Tester (2) \\
\hline SOC & $79(96.34 \%)$ & $3(3.66 \%)$ \\
\hline Dosage level 1 & $30(36.15 \%)$ & $53(63.86 \%)$ \\
\hline Dosage level 2 & $42(50.6 \%)$ & $41(49.4 \%)$
\end{tabular}

Note: Table 4.12 shows the amount of participants in each group, the Standard of Care and each dosage level group in the combined Experimental group, who had been previously diagnosed as $\mathrm{HIV}+$. Those who had been previously diagnosed are listed as Past tester, and those who were newly diagnosed are listed as New tester $(N=248)$.

As mentioned before, not all testers in this sample reportedly were newly diagnosed as HIV+, and tester status (New tester/newly diagnosed versus Past tester/previously diagnosed) was not evenly distributed among the study groups. The total number of Past testers for the whole sample was 97 (39.11\%), and the dates of original HIV+ tests are listed in table 4.13.

Table 4.13 Past Tester Diagnosis Dates

\begin{tabular}{cc}
\hline Group & Frequency \\
\hline Within same year (2015) & 7 \\
& $(7.2 \%)$ \\
\hline $2013-2014$ & 32 \\
$2010-2012$ & $(33 \%)$ \\
\hline \multirow{2}{*}{ Before 2010 (1996-2009) } & 26 \\
& $(26.80 \%)$ \\
\hline
\end{tabular}

Note: Table 4.13 shows the date ranges for those previously diagnosed as HIV+, whom are listed as past testers $(N=97)$.

Even among new testers, a greater percentage of participants reported violence upon partner notification in the Standard of Care group (6/75 or $8 \%$ ) compared to the combined Experimental group ( $3 / 70$ or $4 \%$ ), but the difference 
was non-significant $\left(X^{2}{ }_{(1)}=.86, p=.496\right)$. Among the total group of past testers, 3 reported violence upon partner notification ( 1 from the Standard of Care group, and 2 from the combined Experimental group). Of the total number of participants who reported violence upon partner notification, (3/15) $20 \%$ were past testers. However, when removing those who had tested longer ago than two years from analysis (see table 4.13), Pearson's Chi square showed that significantly more participants in the Standard of Care group (9/79 or $11.4 \%$ ) compared to the combined Experimental group (4/106 or 3.77\%) received violence upon partner notification $\left(X_{(1)}^{2}=.4 .02, p=.045\right)$.

Additionally, for past testers, the number of participants who reported feeling safe getting to medical appointments (96/97 or $98.97 \%$ ) dropped significantly by post-test (35/97 or $36.08 \%$ ). McNemar showed the difference was also significant among this group $\left(X^{2}{ }_{(1)}=.610, p=.000\right)$. While the pre-post differences remained significant among new testers (pre-test=140/151 or $92.72 \%$; post-test $=82 / 149$ or $\left.55.03 \%) ;\left(X^{2}(1)=.01, p=.000\right)\right)$, the most extreme change occurred between the pre-test and post-test for the past testers.

\section{Anticipated trouble because of HIV status}

However, other items indicate that past testers also anticipated they would experience difficulties because of their status (after this HIV test). For all participants responding to the pre-test item, Because of HIV+ status you will be in trouble with your partner, a higher percentage $(50 / 160$ or $31.3 \%)$ of people in the combined Experimental group answered yes, compared to those who answered 
yes in the Standard of Care group (23/78 or $29.49 \%)$. Among the combined Experimental group, percentages for those answering affirmative to this item were slightly higher for new testers (24/70 or $34.29 \%)$ than past testers $(26 / 90$ or 28.88\%). However, when controlling for the item Because of your HIV status you will be in trouble with your partner, the difference between violence upon partner notification of serostatus remained significantly higher among those who reported they would not be in trouble with their partner in the Standard of Care compared to those in the combined Experimental group $\left(X^{2}(1)=9.95, p=.003\right)$. Because the number of past testers is not evenly distributed among the research groups (far fewer in the standard of Care versus the combined Experimental group), numbers for past testers were not sufficiently comparable to test differences in linkage between groups. Thus, items that show participants' anticipated difficulties because of their HIV status may be a better indictor for comparing the impact of violence upon partner notification.

Some studies compare the amount of re-assault experienced between those who have left the abusive relationship compared to those who remain in the abusive relationship. In this study, 11/248 (4.44\%) participants had left their partners upon pre-test data collection. Of those who had left their partner, 0 received violence upon partner notification of serostatus, but 2 of those had reported they would be in trouble with their partner because of their HIV status.

Finally, more people in the combined Experimental group (25/50 or $50 \%$ ) who answered yes to the item You will be in trouble with you partner because of your HIV status linked to care than those who answered yes and linked to care in 
the Standard of Care group (6/23 or $23.2 \%$ ), and Pearson's Chi square showed it was trending towards significant $\left(X_{(1)}{ }_{(1)}=1.06, p=.055\right)$. 


\section{CHAPTER 5}

\section{DISCUSSION}

Thus far, the linked epidemics of HIV and intimate partner violence (IPV) have been explored and the impact of the HIV-IPV intersection on South African women has been demonstrated. The need for the HIV IPV Risk and Safety (HIRS) protocol has been highlighted, as well as its empirical support and theoretical underpinnings. The methodology and results of the study have also been presented. This final chapter will synthesize the results by comparing findings with current literature and postulating their practical significance towards research and practice. The majority of this chapter concentrates on interpreting the relevance of the results as contextualized within the extant literature and is organized by descriptive findings and research questions. The chapter concludes by discussing study strengths and limitations, an appraisal of implications for practice, recommendations for future research, and a conclusive statement about the study's significance.

\subsection{Descriptive Findings}

IPV Prevalence 
Unlike many studies, IPV eligibility for this study included not only participants who had experienced physical and sexual abuse but also those who had only experienced non-violent control in their relationship. However, participants reporting only non-violent control must have reported at least two forms of non-violent control for eligibility (compared to those with physical or sexual abuse only requiring one form of physical or sexual abuse for eligibility). Many studies on IPV only include those with physical or sexual abuse (Abramsky et al., 2011; Abramsky et al., 2012). Although comparisons are rendered difficult because there is no gold standard for measuring IPV in general (Abramsky et al., 2011), some comparisons may be firmly drawn. This study found that of the 446 participants recruited, 191 did not report IPV, but the other $255(57.18 \%)$ did report IPV. Of those who reported some form of IPV, $167(65.49 \%)$ had at least one form of physical or sexual abuse in the last year. That is an overall prevalence of physical or sexual IPV in the last year of $37.44 \%$. Of those who met IPV eligibility, $99.19 \%$ of the total sample reported having experienced at least one form of non-violent control in their relationship within the past year, and non-violent control experiences were reportedly very similar between study groups. Among the total sample, $40.73 \%$ reported having experienced at least one form of physical abuse in their relationship in the last year, and percentages were significantly higher among the combined Experimental group (45.18\%) compared to the Standard of Care group (31.71\%). Also in the total sample, $46.77 \%$ reported having experienced some form of sexual abuse in their current relationship in the past year, and percentages were higher among participants in 
the combined Experimental group (48.8\%) compared to the Standard of Care (42.68\%). Thus, baseline levels (at pre-test) of IPV were higher for those in the combined Experimental group than the Standard of Care group.

For the overall sample, these prevalence rates are comparable to that found in the literature, with some being higher and others being lower. Comparisons with household surveys show general IPV prevalence rates to be lower than those among women living with HIV. For example, while this study found $37 \%$ of participants had experienced physical or sexual IPV in the last year, Abramsky et al. (2012) report a $27 \%$ rate among women in an HIV prevalent area in sub-Saharan Africa; another household study of men reporting IPV perpetration in the last year showed a prevalence of $29.6 \%$ for rape and $30.7 \%$ for physical abuse (Jewkes et al., 2011), and this was similar to another South African study reporting $27.5 \%$ of men confessed to perpetrating physical abuse in their current relationship (Gupta et al. as cited in Peltzer et al., 2013).

Among those testing HIV+ in South Africa, Dunkle et al. (2004) reported an IPV prevalence of $40.2 \%$ of physical or sexual assault in the last year among a slightly younger age group presenting in an antenatal clinic in the same province as this study. Most contrasting with the current finding of $37 \%$ was a study of young women (aged 16-24 years) in a peri-urban setting in Western Cape, South Africa. This study used the WHO violence against women instrument and discovered that $86 \%$ of the sample had experienced physical or sexual IPV in the last year (Zembe, Townsend, Thorson, Silberschmidt \& Ekstrom, 2015). 
To compare these rates internationally, Maman et al. (2002) report women testing HIV+ during VCT in Tanzania are more than twice as likely to report physical or sexual IPV in their current relationship than those testing HIV-, and a Ugandan study found $63 \%$ of women testing HIV+ had experienced IPV "at some point" before HIV testing (Kouyoumdjian et al., 2013, pg. 166). A systematic review over literature in the United States, addressing the HIV-IPV link, found up to $28 \%$ of HIV+ women had experienced physical IPV in their current relationship (Gielen et al., 2007). Rates were also higher among women in Tanzania and Ethiopia with the latter reporting $54 \%$ had experienced physical or sexual violence in the last year (Garcia-Moreno, Hansen, Ellsberg, Heise, \& Watts, 2005).

Rates of emotional abuse, while reported less often, have been cited as higher than those of physical and sexual abuse, and this was consistent with the current study's findings. For all 10 countries in the aforementioned multi-country study conducted by the World Health Organization, $20-75 \%$ of respondents had reportedly experienced one or more act of controlling behavior by their partner (Garcia-Moreno et al., 2005). Interestingly, one study found only $43.7 \%$ of women sampled in Gauteng province reported having received emotional abuse, but $65.2 \%$ of men sampled reported having perpetrated it (Rees, Zweigenthal \& Joyner, 2014). Because rates of emotional abuse may be underreported in South Africa due to cultural complicity of gender inequalities, information procured in this study (through safe, confidential and supportive contact) generates new knowledge regarding emotional IPV prevalence rates among those testing HIV+. 
Tester Status: previous or new diagnosis

Of the 248 participants included in this study's pre-test only analyses, 97 (39.1\%) had previously tested HIV+ and were labeled past testers. It is unknown how many times those previously diagnosed had undergone testing before, or how many had already notified their partner of a positive serostatus. For those previously testing HIV+, dates of initial diagnosis ranged from year 1996-2015 with $7.2 \%$ having been diagnosed the same year as this study (2015), 33\% some time in the two years before, and almost $60 \%$ testing some time between 1996 2010. The remainder of the sample (151/248 or $60.9 \%)$ was newly diagnosed as $\mathrm{HIV}+$ (about one day prior to study administration) and was labeled new testers. This is a relatively high amount of past testers. Some sources report sample percentages of those previously diagnosed, and others fail to notate such information. Van Zyl et al. (2015) found $4.1 \%$ of their sample had previously tested HIV+; Larson et al. (2010) found 6.5\%; and Mabuto et al. (2014) found $2 \%$.

It is not known exactly why the numbers of those previously diagnosed are so high in the current study, but the most viable explanation is that this study allowed a more comprehensive inclusion criterion. Results are a likely more accurate representation of who was utilizing mobile testing at the time and location of study recruitment. Other studies may have excluded this sub-group because they were seen as non-representative of the targeted sample for research questions. For example, Wagman et al. (2015) allowed this sub- 
population to remain in their study on HIV and IPV in Uganda, but Maman et al. (2014) did not allow this sub-population in their study of HIV and IPV in Durban, South Africa (as cited in Kennedy et al., 2015). Although including this group caused an unequal distribution, muddling group comparisons and obfuscating findings, through the participation of this sub-group new information was observed about the on-going role of perceived danger related to serostatus for those previously diagnosed as HIV+.

The majority of past testers reportedly sought mobile HCT to obtain their CD4 cell counts. This suggests something motivated them to check their CD4 levels—i.e. changes in physical health or health maintenance in general-but likely indicates these individuals were not currently engaged in care elsewhere. Because those previously diagnosed as HIV+ were shown at baseline data collection to perceive risk levels commensurate to those recently testing HIV+ and because greater attention is needed for those who "are not yet eligible for ART" (Mugglin et al., 2012, pg. 1509), this sub-group deserves greater attention in future studies. Results for each of these groups are discussed and compared in greater detail throughout this chapter.

\subsection{Demographic Factors}

The mean age of this sample was 33 years, and the median was 32 years. These numbers are similar to other studies (Van Zyl et al., 2015) with some being slightly lower (Mabuto et al. 2014) and others being slightly higher (Adams et al., 2011). Other studies had samples of similar racial make-up to the current 
study. $100 \%$ of this study identified as black South African, consistent with other studies in South Africa on voluntary counseling and testing (Kilembe et al., 2015), IPV services (Adams et al., 2011) and general HIV prevalence (Kenyon, Buyze, \& Colebunders, 2013). It is not surprising that a study serving Gauteng residents would include such high percentages of black South Africans given the province is $77.4 \%$ black South African (Statistics South Africa, 2012). Given the comparability of this study's demographics and IPV prevalence, the study's sample resembles the demographic population from which participants were recruited.

\subsection{Contribution of Psychometric Instruments}

HIV IPV Risk and Safety scale

The HIV IPV Risk and Safety (HIRS) scale is a 10-item psychometric tool developed to uniquely assess for danger associated with HIV testing, HIV serostatus, and risks associated with linkage to care and retention in HIV care among those already experiencing IPV in their relationship. This tool was shown to have good reliability and approaching sufficient validity. Future use of the scale should involve minor refinement to improve its validity.

The creation and use of this instrument allowed trends to be uncovered in danger associated with the HIV-IPV intersection. This tool is to be used specifically with individuals already testing positive for both HIV and IPV. Given the multidirectional nature of HIV and IPV, the purpose of the tool is two-fold: to measure how IPV may create a barrier for HIV care and how an HIV+ diagnosis 
may contribute to further risks for IPV. Results from this tool help compare perceptions of danger immediately following an HIV diagnosis to perceptions 30 days after a diagnosis, as well as between groups receiving the HIRS protocol and those only recceing the standard of care. Hale \& Vazquez (2011) highlight the need for information to be generated on abuse before and after an HIV diagnosis, as this information will help explain the nature of violence particular to the HIV-IPV intersection. Thus, this tool is a first effort towards filling a significant gap in the literature.

\section{Danger Indicator}

The psychometric instrument, the Danger Indicator (DI), is a 45-item scale used to measure risk and danger associated with the HIV-IPV intersection. Through Exploratory Factor Analysis, this scale was shown to be an appropriate indicator of danger levels related to intimate partner violence for those who are $\mathrm{HIV+;}$ yet, the scale was also shown to need further refinement to be a more valid instrument. Although this scale includes measurements of non-violent control, physical and sexual violence, vulnerability for any relationship violence, safety and risks related to serostatus, and likelihood for femicide, the latent variable was physical assault. Hence, danger predictions within this study may be more accurate for physical assault rather than sexual or psychological abuse. Each of the aforementioned areas were included to create a tool that would comprehensively assess risk for IPV reoccurrence, risk for fatality and protective factors that may mitigate the IPV (Campbell, 2004), as each of these areas 
should be considered in order for an IPV assessment tool to provide an accurate global assessment. Therefore, future studies should continue to refine the tool so that it is a more valid measurement for all the listed areas of abuse.

Though the tool was found to be appropriate for assessing danger levels, it should be further refined to also consider frequency and severity of past abuse as a more accurate predictor of various types of IPV (Hardesty et al., 2015). An original version of one sub-scale within the Danger Indicator typically is used with a calendar so that the frequency of assaults may be factored into the risk equation. This is an important component to predicting future assault and should be integrated into the Danger Indicator in future use.

This tool is an important contribution to the HIV-IPV knowledge base. Safety planning for risks relevant to the HIV-IPV intersection relies on an accurate assessment of risks that are population-specific. Scores from the Danger Indicator were used as a necessary tributary to inform safety planning so that the two tools were used as one iterative process for mitigating IPV related to serostatus. To date, no tool could be found that measures global scores of danger relevant to the HIV-IPV intersection.

\subsection{Research Question 1A}

\section{Risk \& Safety Scores between Groups}

For research question $1 \mathrm{~A}$, comparing participants' mean difference scores on the HIV IPV Risk and Safety (HIRS) scale, no significant differences were found between the Standard of Care group and combined Experimental group. 
Those in the combined Experimental group had slightly higher mean scores than the Standard of Care group at pre-test and post-test. However, differences were slight and all non-significant. While this study hypothesized that there would be a reduction in mean safety scores (increase in perceived safety) for the combined Experimental group, the null hypothesis demonstrates that this protocol did not incite more risk among those in the combined Experimental group as indicated by participant's perceived risk levels. There is some debate about the best way to measure perceptions of safety among IPV victims (Cattaneo et al., 2007; Harding \& Helweg-Larsen, 2008), calling to question the validity of self-reports. Some of the relevant ways to measure perceptions of safety and their significance are explored further throughout this chapter.

One consideration for the HIRS results is that participants were asked about IPV occurrence at pre-test only and before any received psychoeducation about IPV. Given the degree of cultural acceptance of violence in South Africa, a small increase in risk scores, even if non-significant, could reflect some change in awareness of IPV-related issues. Data collection relied on self-report only, and sources suggest that when corroborated with other collateral sources, rates may be inconsistent if participants are not familiar with recognizing IPV in their relationship (Rees et al., 2014).

Another consideration is this study's short follow-up period (of only one month) may have been too brief to capture the possible reductions in perceived risk that could be experienced as a longer-term result of safety plans or safety strategizing. While Kendall et al. (2009) found participant safety remained 
consistent between telephone follow-up periods of 2 days and 2, 6 and 12 weeks, other examples used longer follow-up periods to uncover increases in perceived safety. For example, when Peltzer et al. (2013) conducted a pre-test post-test analysis of the effectiveness of orders of protections for victims in South Africa, pre-test data were collected immediately following the receipt of an order of protection and then post-test data 6 months later. They observed a significant reduction in IPV at the 6-month mark and found certain forms of IPV, like stalking, played a more active role during the protective order process. This indicates there could be an increase in danger or perceived risk at the time of employing this legal strategy but reduced danger or perceived risk months after doing so. Matseke et al. (2013) also collected data on pre-post Danger Assessment scores, after only a 20-minute safety planning session, and found significant drops in danger but with a 3-month follow-up period. Thus, a longer follow-up period could capture changes only observable farther into the use of a strategy and provide further insight into the effectiveness of safety strategies that take longer to deploy. Two theoretical models could be applied to understand the change process captured in this study, the Transtheoretical Model (TTM) and Lempert's (1996) stages.

According to the TTM, most behavioral change occurs in longer strides than one month, but shifts in cognitive states lead up to such changes. TTM posits that behavioral change is contingent upon a series of cognitive shifts such as alterations in attitudes and decisions. Therefore, change is hypothesized as occurring over time or in stages rather than abruptly or all at once. An Ugandan 
study, utilizing the TTM with abused women and public health programs, found that individuals who are in the precontemplation stage of change do not intend to change in the next 6 months, but those who are in contemplation stage do intend to change in the next 6 months (Wagman et al., 2013). If the current study reached more individuals in the precontemplation stage of change, it is likely that behavioral change would be delayed but changes in perception could be detected sooner as an individual begins to shift into the contemplation stage. A follow-up period of 6 months to one year would show the results of more selfprotective action, rather than shifts in perception occurring earlier on in the change process.

This study worked from the assumption that an HIV+ diagnosis alone would not sufficiently lead to changes in behavior (Bundy, 2004), but those receiving the experimental components of the protocol would experience an accelerated change rate due to alterations in subjective norms around IPV. It is not yet known how much the role of IPV impacted the rate of such a change, and future studies could include more of the TTM as part of the protocol as well as compare change among those receiving HIV diagnoses without IPV to understand if psychometric tools such as the HIRS are detecting changes in perception-indicative of the change process-or actual increases in risk that could occur when a safety strategy is initiated (Matseke et al., 2013).

The second theoretical model that could apply, and shows the current study's findings challenge that of the Ugandan study, is Lempert's (1996) theory describing the three linear stages a woman goes through in responding to abuse 
in her relationship. The first stage involves a woman's attempt to make the abuse invisible and essentially cover it up. The second stage involves both the woman and the perpetrator acting to "contain the violence and preserve personal agency" (Wright, Kiguwa \& Potter, 2007, pg. 618). During this stage, a woman may engage in placating behaviors or attempt to negotiate for non-violence. Strategies used in this stage vary depending on each woman's meaning making of the violence and her available resources. The final stage involves attempts to make the violence more visible through consultation with family, friends, legal and community resources. At this point, the woman more clearly identifies the ramifications of the abuse and the potential to reclaim power by accessing available resources. Through these resources, she may be empowered to "reestablish her own needs, wishes, and eventual departure" [from the relationship] (Kirkwood \& Lempert as cited in Wright et al., pg. 619).

Lempert's theory has been used to describe the experiences of women accessing domestic violence shelters in Johannesburg, South Africa. Results from a qualitative study were found to be consistent with Lempert's theory. Whereas TTM explains the general process for change-making, Lempert's theory illustrates a linear progression women may go through as they travel along the path of an IPV relationship. It is difficult to ascertain where exactly participants of this study would have largely been plotted along this theoretical trajectory, but in light of the high numbers of those utilizing public (versus private) safety strategies (explained under Feasibility), according to this theory it would either be assumed that many women were already in the final stage of this three-part 
stage or that the intervention itself acted as a catalyst, quickening the change process and thrusting participants into a state of action (stage 3).

Given the short follow-up period and limited items asked about readiness to change, conclusions about the impact of study participation on the change process as well as how the change process impacts participant safety remain nebulous. Because this study focused on safely improving HIV outcomes (linkage to care) and not IPV outcomes, the most important take-away of these results is that those receiving the HIRS protocol did not suffer as a direct result of protocol receipt. Rather, those in the experimental group maintained risk levels parallel to those in the Standard of Care group, especially considering their pretest risk levels were slightly elevated. However, it is hypothesized that a longer follow-up period may uncover greater reductions in risks for those receiving the protocol, with the 30-day follow-up mark being muddled by women transitioning either cognitively or behaviorally into various stages of change. Yet to be determined is how exactly these stages of change may typically evolve for women impacted by the HIV-IPV link. This study's working assumption is that receipt of the HIRS protocol expedites the change process, as evidenced by the significant number of participants in the combined Experimental group who accessed public consultation strategies compared to the Standard of Care group. Likely, the protocol galvanized participants to take action, thrusting them into a different stage of change or Lempert's stage, the latter being an imperfect theory for those experiencing both HIV and IPV. Nonetheless, the amplified results of their actions would likely only be observable at a later date. Because this was 
anticipated to some degree, participants were asked if it would be safe to be contacted again at a later date of 1, 2 or 3 months (see Feasibility, domain 4). Findings from this question will inform future studies, possibly a Phase II trial, on the feasibility of extending the follow-up period.

\subsection{Research Question 1B}

Risk and Safety Scores by Dosage Level

For research question 1B, comparing participant's mean difference scores on the HIV IPV Risk and Safety (HIRS) scale, no significant differences were found between Dosage level 1 and Dosage level 2. While it was hypothesized that those in Dosage level 2 would report reductions in mean HIRS scores (increased safety), the two groups strikingly had the same mean scores for pretest and post-test. Thus, the null hypothesis was supported and the extra dosage level did not alter perceptions of safety among those in Dosage level 2 . It is noteworthy that the higher dosage level did not contribute to higher danger among participants.

\subsection{Research Question 2}

Linkage to Care

For research question 2, it was hypothesized that those in the combined Experimental group would have a higher number of individuals link to care at a faster rate (within 30 days). Although a higher percentage of participants in the combined Experimental group linked to care compared to the Standard of Care, 
the difference was non-significant, and it took slightly longer for those in the combined Experimental group to link to care (about 13 days) than those in the Standard of Care group (about 12 days).

The linkage rate of 12-13 days is consistent with van Zyl et al. (2015), but the overall percentages of participants linked in this study were lower. Van Zyl et al. used the same standard of care as this study but did so in both urban and rural settings, with a different recruitment period, using a 31-day linkage mark and not controlling for IPV. Their study found higher linkage rates for almost all age groups except those aged $\leq 23$ years. The current study found an overall linkage rate (for the total sample) of $41.6 \%$ within 30 days and $45.8 \%$ by post-test (with only $9 \%$ of those linking after 30 days, $M=13.11$ ). Comparatively, van $Z y l$ et al. (2015) reported $54 \%$ of their female sample linked to care and most did so within 14 days. The current study found the highest linkage rate among those aged $23-32$ years $(51.81 \%)$, and the lowest linkage rates for those aged $\leq 23$ years (35.9\%). Van Zyl et al. also found the lowest linkage rates among those aged $<23$ years with their urban group (in a similar area as this study) linking only $32.9 \%$ of participants, which was lower than this study's Standard of Care (35.7\%) and combined Experimental group (36\%). This underscores that those aged $\leq 23$ years need greater attention in future research and practice.

In the current study, those aged 23-32 had very similar linkage rates among the Standard of Care, combined Experimental group and those in the van Zyl et al. (2015) study. Though differences were negligible, the Standard of Care 
in this study had the highest rate of $53.33 \%$, followed by $53.1 \%$ in van Zyl et al., and $51 \%$ in the combined Experimental group of the current study.

The biggest difference in linkage rates, within the current study and compared to van Zyl et al. (2015), was found among those aged 33-43 years. In the current study, among this age group only about $22 \%$ of those in the Standard of Care group linked and about $47 \%$ of those in the combined Experimental group linked. Both of these numbers are lower than the $61.8 \%$ found by van Zyl et al. among this age group.

Additionally, for those answering yes to the item Because of your HIV status you will you will be in trouble with your partner, significantly more people answered in the affirmative but still linked to care among the combined Experimental group (50\%) compared to those answering in the affirmative but still linking to care among the Standard of Care group (23.1\%). This could suggest that the protocol was more effective in linking those who felt greater risk related to their serostatus.

No other studies could be found reporting on linkage rates for mobile testers with IPV. The novelty of this study makes it difficult to compare linkage rates with existing studies that do not control for IPV, as other samples naturally would have contained participants experiencing IPV (as evidence by the IPV prevalence rate among those testing HIV+). Therefore, these findings reveal trends previously unknown and mark a significant contribution to the literature on linkage to care after mobile HCT. 
Given that women aged 30-34 have the highest HIV prevalence rate (UNAIDS, 2012), the high linkage rates among this age group in the combined Experimental group show promise that the protocol can effectively promote linkage to care for women in this age group. Further, the salient need for interventions addressing the HIV-IPV intersection is highlighted by the contrastingly lower linkage rates among those in this age group in the Standard of Care, coupled with the volume of participants in the study who were around this age (mean was about 33 years old).

\subsection{Research Question 3A}

\section{Satisfaction between Groups}

Research question $3 \mathrm{~A}$ hypothesized that those in the combined Experimental group would have higher satisfaction scores than those in the Standard of Care group. Although those in the combined Experimental group did have higher percentages of those reporting they were satisfied, the difference was non-significant. For the total sample, about $93 \%$ of participants reported the information they received was helpful; about $97 \%$ of participants reported it was helpful to be asked about the difficulties in their relationship, and a little over $95 \%$ reported they did not regret talking about the difficulties in their relationship. For both of the first two categories, those in the combined Experimental group $(92.5 \% ; 97.5 \%)$ reported being slightly more satisfied than those in the Standard of Care group (92.4\%; $96.2 \%)$, and only slightly more participants in the 
combined Experimental group (about 5\%) reported feeling regret compared to those in the Standard of Care group (about $4 \%$ ).

These numbers illustrate that the HIRS protocol was overwhelmingly wellreceived by participants regardless of group allocation. This is an important finding because it shows that despite having not received the experimental components of the study, those in the Standard of Care group were still satisfied with the services received and those who received in-depth safety planning were not more regretful about discussing their difficulties at such length. Hence, participants in either group perceived the benefits of study participation far outweighed the regrets.

The Appollis et al. (2015) systematic review over harms, benefits \& regrets experienced while being surveyed about abuse showed the current study's results were standard by comparison. The current study found $5 \%$ total reported regretting participation, and other studies found anywhere from 4-6\% reported regret, but some reported about $20 \%$ of participants experiencing drawback or discomfort (Carter-Visscher as cited in Appollis et al., 2015).

The current study operationalized the benefit of study participation as affirmative responses to items over the helpfulness of services, but some studies operationalize benefit through items directly asking if study participation was beneficial. For the current study, only $3 \%$ reported services were not beneficial. This compares favorably, as one study found $25 \%$ did not report a positive experience. It is also important to consider that by asking about the helpfulness of services overall, participants may have been considering additionally the 
helpfulness of HCT services and not merely those of study participation. Van Zyl \& Barney (2014) reported only $1.8 \%$ of black participants had been dissatisfied with their program, which was used as part of the standard of care for this study. Percentages of dissatisfaction were only marginally higher in this study, and satisfaction $(97 \%)$ far outweighed regrets $(5 \%)$.

\subsection{Research Question 3B}

Satisfaction, Acceptability \& Feasibility by Dosage Level

\section{Satisfaction}

For research question 3B, the impact of the higher dosage levels on participant's satisfaction scores (which included items over feasibility and acceptability) was hypothesized to be unknown. While a higher percentage of participants in Dosage level $2(93.7 \%)$ compared to dosage level $1(91.4 \%)$ reported that overall they were more satisfied with the services they received, the difference was non-significant. Among dosage groups, the same percentage of participants $(97.5 \%)$ reported it was helpful to be asked about the difficulties in their relationship, but more participants in Dosage level 2 (96.2\%) compared to Dosage level $1(93.8 \%)$ reported they did not regret talking about the difficulties in their relationship.

All of these numbers are practically significant given the dissent among researchers about the risk/benefit ratio of involvement in research on abuse. The high percentage of satisfaction and low percentage of regret among those in the 
Standard of Care group demonstrates that individuals currently experiencing IPV and recently testing HIV+ find participation in such a protocol (largely amounting to IPV screening and continued contact for this group) satisfactory and not inducing of psychological distress (although additional probing of baseline psychological distress at pre-test would assist with greater certainty).

\section{Acceptability}

Items related to feasibility and acceptability were given only to the Experimental group and targeted the acceptability and feasibility of the protocolparticipants informed of their Danger Indicator results (risk assessment) and the creation of a personalized safety plan. Differences in experiences were investigated between the dosage levels, and for all three areas assessed, those in Dosage level 2 had higher percentages of acceptability. Although a higher percentage of participants in Dosage level $2(81 \%)$ compared to Dosage level 1 (79\%) reported they found the safety plan helpful, the difference was nonsignificant. Unanimously, those receiving Dosage level 2 (100\%) reported they would recommend the safety plan for a friend, and most participants in Dosage level $1(95 \%)$ also reported they would recommend a safety plan for a friend, but the difference was also non-significant. Finally, a higher but non-significant number of participants in Dosage level 2 (82.3\%) compared to Dosage level 1 (79\%) reported they had used at least one safety strategy.

These numbers are practically significant given that no information could be found in the current literature on the helpfulness of HIV-IPV specific safety 
strategies or the perceived helpfulness of HIV-IPV specific safety plans. These high percentages suggest that women living with HIV and experiencing IPV find safety planning to be helpful and safety strategies acceptable enough for use, and they would especially recommend safety plans for friends.

Comparisons of perceived safety planning helpfulness and safety strategy usage are difficult because only literature on IPV safety planning could be found, and literature in this area is not well evolved. Thus, these findings contribute not only to the knowledge base on the HIV-IPV intersection but also add to the IPV knowledge base by showing perceptions of safety planning helpfulness and safety strategy usage. Another caveat to comparing these findings to extant knowledge is that most comparison studies present samples of women already sheltered (those who have left the abusive partner) and studies reporting on strategy usage and perceived helpfulness do so for each safety strategy rather than safety plans as whole (Parker \& Gielen, 2014). These issues aside, this section will compare overall helpfulness of the safety plan to safety strategies, and usage percentages for safety strategies are listed below under feasibility.

For the total sample in the current study, $81 \%$ reported finding the safety plan helpful, and $97.5 \%$ said they would recommend one for a friend. Parker \& Gielen (2014) show participants, on average, report informal and formal networks (friends and family and medical and health professionals) are more helpful than strategies such as legal services. Concurring, Goodman, Dutton, Weinfurt \& Cook, (2003) reveal the strategy of talking with family or a friend was experienced as particularly helpful in their study. This could explain the high level 
of those who found the safety plan to be helpful in the current study, as legal services were infrequently employed versus talking with family/friends, and medical and mental health professionals were most often employed. Additionally, most studies assessing the helpfulness of a strategy did so using a likert scale and not a dichotomous item. Because these studies reported on mean scores for those scales, comparisons were incompatible (Parker \& Gielen, 2014; Goodman et al., 2003). No information could be found from existing literature about whether or not other participants would recommend safety planning or certain strategies for friends. However, this question could be similar to a question often asked in other studies on abuse; generally, participants are asked if they find the topic of the study to be important.

\section{Feasibility}

Assessing feasibility entailed several layers of assessment, so domains were created to help demarcate each area. Domain 1 considered the time to administer the study (attempts to reach participant by telephonic medium); Domain 2 considered the length of the intervention to determine the cost in staff time; Domain 3 considered the usefulness of the safety plan as a whole and the amount of strategies used; Finally, domain 4 considered any risks experienced as a result of receiving services.

\section{Feasibility: Domain 1 Time to Administer}

For the combined Experimental group, it took an average of almost 4 attempts to reach participants for the pre-test, and an average of about 6 
attempts to reach participants for post-test. For those in Dosage level 2, it took on average almost 13 attempts to reach participants for follow-up safety plans. Given that most participants were reachable not only for pre-tests but also posttests and some for follow-up safety plans, participant reachability compares extremely favorably with call centre results with a similar population. Van Zyl et al., (2015), tracked linkage to care for individuals newly diagnosed as HIV+, and used the same Links to Care program as utilized in this study's standard of care. Their study found, of those who did not link to care $(N=255), 64.7 \%$ in the urban setting were not reachable by phone despite many attempts to reach them. This dramatic comparison to the current study's success in reaching participants lends only a speculative explanation: participants of this study could have increased motivation or need for help given the IPV in their relationships.

\section{Feasibility: Domain 2 Length of Intervention}

For the combined Experimental group, it took an average of about 10 minutes to administer the pre-test (Danger Indicator) portion of the initial phone call; an average of about 9 minutes to conduct the initial safety plan; and an average of 6.5 minutes to administer the post-test. For those in Dosage level 2, it took an average of 4.8 minutes to conduct the follow-up safety plan. In sum, the longest portion of the protocol was the pre-test followed by the initial safety plan, with the post-test taking up less time than the pre-test, and the follow-up safety plan taking about half as long as the initial safety plan. In light of the increase in acceptability and satisfaction found among those in Dosage level 2 in tandem 
with the small amount of time it took to administer the follow-up safety plan, at first appearance the follow-up safety plan appears to be a low-cost component relative to operational costs. Conversely, the gross amount of attempts it took to reach participants for the follow-up safety plan portends challenges in staff time management and, therefore, possible superfluity given the non-significant differences between groups.

The HIRS protocol was intentionally developed to be a short tool that could be feasibly used in a high-demand, resource-limited country, as this setting poses the most time restrictions. For an HCT service to entertain the use of such a protocol, it would have to be brief. The protocol also intentionally narrows its focus to strategies relevant to the HIV-IPV intersection and does not attempt to tackle general IPV, which would likely require a longer interview time, follow-up period and greater community involvement in order to impact greater change. In the Goodman et al. (2003) study of the development of the IPV Strategy Index, interviews were also held over the phone but each one took an average of 45-60 minutes. Another study specifically on IPV safety planning, took about one hour to administer (Glass et al., 2010). When combining the Danger Indicator component of the protocol with the safety plan, the protocol took about 20 minutes to administer. This highlights the achieved brevity for which the protocol was intended.

Feasibility: Domain 3 Usefulness of Safety Plan \& Strategies

Items for this domain considered if the safety plan was feasible to use and, if so, how many of the strategies were used. While a previous item under 
acceptability uncovered higher percentages of those in the combined Experimental group found the safety plan to be helpful, a smaller percentage actually reported using the safety plan. A higher percentage of participants in Dosage level $2(63.3 \%)$ reported using the safety plan and used more safety strategies $(M=2.18$ strategies used) compared to those in Dosage level $1(60.5 \%)$ $(M=1.76$ strategies used) respectively, but the differences were non-significant.

The number of individuals who reported having used a safety plan compares well with Glass et al. (2010) who reported $60 \%$ of their sample had made a safety plan. Further comparisons consider the use of each strategy within an overall safety plan and are organized by the most popularly utilized strategy category of Consultation strategies \& Planning Strategies to the least utilized strategy categories of Private Strategies of Resistance \& Placation.

As an overview, the current study found the top four most utilized strategies were the Consultation strategies of talking with a medical professional (44\%), friend/family member (44\%), counselor/mental health professional $(32 \%)$, and clergy member (23\%). Strategies utilized most after these were the Planning strategies of creating a plan to safely take HIV medication (16\%) and safely access HIV care (15\%); and General Safety Planning strategies (thinking ahead and doing things like hiding money, keeping important phone numbers with you or other behaviors to help prepare for an emergency) (12.5\%). Finally, accessing formal IPV services (9\%) was utilized more than legal services (including police, attorneys, orders of protections and both were also considered consultation strategies) (7.5\%), and Resistance strategies (fighting back, trying to end the 
relationship, running and hiding when violence occurs) (5\%) and Pacification strategies (trying to avoid the violence with activities like avoiding partner at certain times, doing whatever partner wants you to do, trying to keep things quiet or a secret from partner) (4\%), also considered private strategies, were utilized the least.

\section{Consultation strategies}

$80 \%$ of participants in the combined Experimental group reported using some type of consultation strategy. If including those in the Standard of Care group (who were deemed contaminating cases), $84 \%$ of the total sample reported using some type of consultation strategy, and usage numbers only increased with each dosage level: Dosage level 1 at $77.78 \%$ usage and Dosage level 2 at $82.28 \%$ usage. With $84 \%$ of the total sample having accessed some type of consultation strategy to mitigate the IPV, the influx of women testing HIV+ who could benefit from the integration of HIV and IPV services on a regular basis is emphasized. This point is further accentuated by the strategy usage disparity between groups; $80 \%$ of those in the Experimental group used a consultation strategy compared with only $4 \%$ in the Standard of Care group. This demonstrates that participants were significantly more ready and able to take action to protect themselves with receipt of the experimental component of the HIRS protocol. Because women who have experienced IPV are more likely to access health-related services than formal IPV services, especially in developing countries (Chibber \& Krishnan, 2011), the potential for assisting many vulnerable women with a feasible and appropriate protocol such as the HIRS has been 
shown. Consultation strategy usage in this study is comparable to usage percentages cited in other studies, which range between $87.3 \%$ (Coker as cited in Parker \& Gielen, 2014) and 91\% (Glass et al., 2010). Peltzer, Mashego \& Mabeba (2003) show much lower consultation strategy usage in their study, but strategies are separated out into individual groups and so are listed below in corresponding areas.

The percent of participants who used the strategy talking with a medical professional was only slightly higher than other (largely North American) studies have reported but was equivalent to the strategy of talking with a family or friend. The latter informal network, talking with friend or family, is cited as a much more common strategy, and the equivalent usage between these strategies in this study highlights the significant role of medical professionals to potentially assist those with both HIV and IPV. The current study found $44 \%$ of participants had talked with a medical professional, but other studies reported a range from $22 \%$ $36.4 \%$ with less use among individuals in urban areas or among those who identified as persons of color (Glass et al., 2010; Shannon as cited in Parker \& Gielen, 2014). Taking into account the limited services in South Africa (Rees et al., 2014), this usage percentage is quite high, particularly considering a South African study focusing on IPV only found only $15 \%$ of its participants had contacted a medical professional (Peltzer et al., 2003).

The current study's high percentage of participants consulting with medical professionals is consistent with the united message found among varying sources-there is an important need for healthcare workers to be trained 
in IPV response as healthcare sites are accessed by victims more frequently than formal IPV services (Lindhorst et al., 2005). Despite this need, some healthcare workers have left victims feeling judged (Hale \& Vazquez, 2011) or as though they must follow their worker's directive to leave the relationship. One study found only $9.7 \%$ of doctors in South Africa had been trained in IPV response and cites doctors as often holding attitudes of victim blaming while reporting they should be involved in IPV response.

The current study found $44 \%$ of participants had talked with family or friends. Other studies found $69.1 \%$ talked with family, $74.5 \%$ talked with friends (Cooker as cited in Parker \& Gielen, 2014) or $64 \%$ talked with a friend, and $49 \%$ talked with a family member (Glass et al., 2010). As mentioned above, this strategy has been shown to be particularly helpful (Goodman et al., 2003). Moreover, while this category's usage frequency is lower in the current study, by comparison to studies focusing only on IPV, the high usage frequency within this study by comparison to other strategy utilization may explain the high percentages of those who perceived the overall safety plan to be helpful.

IPV is said to not only affect a woman's physical and emotional self but also her spiritual self (Davies \& Dryer, 2014). As a country with a high amount of religious affiliation—about $2 / 3$ of South Africans identify as Christian—the moderate amount of clergy consultation reported in this study is not surprising. $23 \%$ of participants reported utilizing the strategy of talking with clergy, and this was very similar to that found in another South African study (priest-25\%) (Peltzer et al., 2003). Other North American studies cite a range of anywhere 
from $6 \%-27 \%$ of participants using this strategy, with a lower percentage found in some rural areas (El-Khoury \& Wiist as cited in Parker \& Gielen, 2014). Although the current study's numbers hardly show most participants are likely to use this strategy, as Peterson (2009) claims, they do provide support for Peterson's assertion that clergy in South Africa should be aware, trained and ready to respond to IPV victims. Peterson's qualitative study uncovered clergy responses to IPV in South Africa as running the gamut: some identified IPV as a grave problem; others felt ill-equipped to handle it or as though it was not their place to respond to IPV; some even displayed burn out from seemingly incessantly needy victims and clergy member's obligatory helping role. Ultimately, Peterson urges that there is an exigent need for "sound ministry of healing and compassion" within the church not just to respond to victims but also to hold perpetrators accountable. The potentially important role clergy members may play in IPV response was confirmed by almost a quarter of the current sample seeking help through this medium.

The current study found $32 \%$ of participants reported having talked with a counselor or mental health professional. Other IPV studies report anywhere from $29-45.5 \%$ of participants had contacted mental health professionals (Coker \& ElKhoury \& Parker \& Gielen, 2014), and one study separated out various types of mental health professionals utilized by South African survivors with $33 \%$ contacting a social worker before anyone else, followed by a psychologist $(7 \%)$ and traditional healer (5\%) (Peltzer et al., 2003). 
These findings, in-part, support the allegation that IPV survivors in South Africa prefer counseling services, but higher rates have been found in other studies in South Africa, ranging from 36\%-45.8\% (Jewkes et al. as cited in Rees et al., 2014), and lower percentages in other studies (therapist-12\%) (Glass et al., 2010). Consultation with a mental health professional was the third most popular strategy employed in the current study. Because of its popularity, a brief review of perception of IPV among mental health professionals in South Africa provides insight into what survivors might experience upon accessing this strategy.

In one exploratory study, the professionals interviewed described IPV survivors as either intentionally remaining in abusive relationships or not having access to services to assist an escape. According to perception, the survivors wanting to remain in abusive relationships allegedly do so "for cultural reasons, economic dependence and fear of stigmatization" (pg. 5577). Perception continues that from this decision survivors typically end up adopting maladaptive coping skills, as a survival mechanism, and ultimately experience impairment in mental well-being. These individuals are said to subsequently suffer from helplessness, submissiveness, low self-esteem and dependence. Conversely, of the many women who want to leave their abusive relationships, many are stymied by their lack of knowledge of community services to assist or by the actual insufficiency of IPV services in their community. A final perception among mental health professionals was the ideology that victims of abuse have a lack of survival skills; this idea diverges from the popularly held belief among IPV 
scholars that survivors are extremely resourceful and persistent in their attempts to protect themselves (Goodman et al., 2003). In summation, the authors of the above study discuss the need for more community-based services that are empowering (Gumani \& Mudhovozi, 2013).

Thus, this depiction of the ideology among mental health professionals, while not generalizable, raises concern for how survivors are received upon deploying this strategy. First, the perception paints a rather binary view of victim experience, one that is bereft of concepts such as love, hope and dreams. Although the authors report that the results were in line with Gelles (1987) findings, which do discuss hope and the possibility of reform among perpetrators (Gumani \& Mudhovozi, 2013), results focused more on the cultural reasons victims remain in relationships rather than the actual role of love.

Literature on IPV in South Africa often does not address these concepts when attempting to explain why victims remain in abusive relationships, and it would enhance cross-cultural understanding for future studies to explore the role of these concepts. A qualitative study by Wright et al. (2007) do show their small sample addressed the theme of "trust and belief in romantic love" as a reason women remain in abusive relationships, but this was coupled with "the sanction of marriage used by male partners to keep women in the relationship" (pg. 627). However, the intimation that women who do remain eventually transform into helpless beings is refuted among IPV scholars (Van Schalkwyk, Boonzaier, \& Gobodo-Madikizela, 2014), which contradicts Walker's (1979) theory of learned helplessness (for which she eventually recanted herself (Goodman et al., 2003)). 
Consequently, it may be extrapolated that more robust IPV training for mental health professionals is needed if the research-to-practice gap may be bridged. Finally, given the discrepancy between IPV ideology among scholars and those in the trenches caring for survivors, the potential weakness of safety planning as a main intervention component may also be deduced. To some extent, the safety plans themselves are only as powerful as the resources within them. This underscores the need for greater community-level IPV initiatives and interventions to work hand in hand with interventions addressing the HIV-IPV intersection.

Results over utilization of IPV services are the most difficult to compare with both domestic and international findings. First, there is a paucity of literature on IPV service utilization in South Africa. Second, North American comparisons cannot be equitably interpreted given the much higher availability of IPV services there, and third, most North American studies include samples of women already residing in domestic violence shelters. Strikingly, despite these issues in data comparison, the current study found that $9 \%$ of the combined Experimental group utilized formal IPV services, and a telephonic cross-sectional population-based survey in the US found only $10.9 \%$ had utilized formal IPV services. As of 2007 , the idea of sheltering abused women in South Africa was cited as being both a relatively new concept and more Western-oriented. Instead, South African culture was said to rely more on the support of extended family, and isolation from extended family was one of the factors contributing to women utilizing the 
resource of a shelter (Wright et al., 2007). Differences in shelter milieus between Western and African shelters are discussed more thoroughly by Wright et al.

Although formal IPV services are cited as inadequate or prohibitively cumbersome to reach in South Africa (as a vestige of the Group Areas Act) (Vetten, 2005), three possible explanations were considered for the comparable usage of this strategy. One is that the problem of IPV may be increasingly destigmatized since the country's adoption of one of the most progressive Domestic Violence Acts in the world (Mogale, Burns \& Richter, 2012), and connected to this could be an increased usage of shelter services among women. An important distinction of non-Westernized shelters reviewed by Wright et al. (2007) was their openness to women who not only wished to end their relationship but also for those who merely wanted a time out or cool down period for those who were in danger but did not want to leave for good. This could allow IPV victims to more fluidly access services if they are perceived as a less dramatic and non-committal option. The final explanation for the high usage of IPV services could be related to the effectiveness of the HIRS safety plan, specifically in light of the organization of the safety plan. Available domestic violence shelters and services were discussed first with participants, and the high usage, in accordance with the concept of primacy within memory recall dynamics (Kahana \& Miller, 2013), could indicate participants recalled this option more readily than they would have if otherwise organized.

For the legal consultation category, $7.5 \%$ of participants had used some type of legal strategy, but this category assessed for strategy usage by lumping 
several legal strategies into one item probing for either police or attorney contact or taking out an order of protection (restraining order, court order or interdict). Peltzer et al. (2013) cite almost 300,000 people applied for an order of protection (OOP) in South Africa in $2009 / 2010$, emphasizing the popularity of this approach in South Africa. Their study, in the Vhembe district, found OOP's to be highly effective in reducing violence. However, this area varies in many ways from areas in the Gauteng province region where the current study's participants were recruited, and Peltzer et al. completed post-test follow-up at the 6 month mark; whereas, the current study only waited about 4 weeks for follow-up. It is possible that a longer follow-up period may procure different findings for the Gauteng area as well. Additionally, because the current study assessed for an aggregate use of any legal strategy, it cannot be ascertained how many who used any legal strategy actually took out an order of protection versus speaking with a lawyer or calling the police. While the number of $7.5 \%$ may sound low, another study found only $1 \%$ of victims had utilized this strategy (Wiist as cited in Parker \& Gielen, 2014) but one study in South Africa cited $27 \%$ had contacted the police (Peltzer et al., 2003), and one in the U.S. showed $23 \%$ had contacted the police (Glass et al., 2010). In contradiction with the current study's findings, Adams et al. (2011) reported that seeking help from the police had the strongest correlation with HIV testing in their sample, but they did not know if the testing or the help-seeking from the police came first.

Perceived helpfulness of police varies among IPV survivors, with sources finding half of participants reporting the police were effective in mitigating the 
violence, but another $17 \%$ reporting they had contributed to worsening the violence. Another study from the same systematic review found that $63 \%$ of participants saw the police helpful but $28 \%$ did not (Davies, Block \& Campbell as cited in Parker \& Gielen, 2014). Although these rates portray some of the experiences of IPV survivors utilizing the police for assistance, a greater depiction of police reception specifically in South Africa is needed to fully contextualize this discussion. Bendall (2010) tells that historically police in South Africa have been known for neglecting matters related to domestic violence, despite the country having one of the most progressive domestic violence acts in the world. Police have often been either unaware of the laws around domestic violence, responded aggressively to calls, refused to charge men for assault or carried similar beliefs and attitudes of gender inequalities as found among IPV perpetrators. It is questionable, therefore, if police would be seen as helpful or harmful to a victim. Further, women with economic dependence on their abusive partner are less likely to contact the police about the abuse (Van Schalkwyk et al., 2014), but this study did not assess participant's economic constitution.

\section{Planning Strategies}

The category of planning strategies included three areas, which are discussed by descending usage popularity. $16 \%$ of participants reported using a planning strategy to safely take HIV medication, and $15 \%$ reported using a planning strategy to safely get to medical appointments. To some extent, these numbers would be expected as higher due to the number of participants who went on to link to care. Nevertheless, it is not surprising that immediately 
following testing, HIV+ participants may not have been able to accurately assess their danger levels, as evidenced by those at pre-test who reported they would feel safe getting to medical appointments compared to those who reported at post-test that they had not in actuality felt safe getting to medical appointments, which increased significantly for all groups.

Only $12.5 \%$ of participants reported using general safety planning strategies. Although Goodman et al. (2003) separated out each of the planning strategies when assessing for usage and perceived helpfulness and included more strategies, usage of planning strategies among their sample ranged from $29.7-62 \%$. Other studies also separated out many of these strategies with one reporting $61 \%$ of participants reporting they kept important phone numbers hidden and $75 \%$ reported they had created an emergency escape plan that included hiding money. All the sources reporting higher numbers were procured from women who were attending for IPV services such as domestic violence agencies or legal aid.

\section{Private Strategies: Resistance \& Placation}

Only $5 \%$ of the combined Experimental group reported using Resistance strategies. This is significantly lower than that reported in some other studies on IPV. One study, having the same sample size as the combined Experimental group in the current study but focusing on battered women already in a shelter, reported $83 \%$ of participants had used this strategy (Van Schalkwyk et al., 2014). More aligned with the current study, another source reported $7.5 \%$ usage of the running and hiding strategy (O'Campo as cited in Parker \& Gielen, 2014). One 
explanation for the low usage numbers of this strategy is the content of the safety plan. Participants were informed during safety planning of the correlation between fighting back strategies and increases in violence. The low usage of this strategy could suggest the effectiveness of this area of the safety plan.

The final type of strategy discussed during safety planning, which was also assessed for in the aggregate, was Placating strategies. The current study found that only $4 \%$ of participants used the placation strategy, but other studies reported $90 \%$ of its participants used this type (Goodkind as cited in Parker \& Gielen, 2014). It is unknown exactly why placation strategies were employed so infrequently in this study, but the qualitative study by van Schalkwyk et al. (2014) over South African survivors in IPV shelters explains that the women in their study, who had already left their abusive partners, reported being much more apt to resist abuse and attempt to shift their sense of power away from the abuser than they were to acquiesce to the abuse.

According to Goodman et al. (2003), placating strategies are used to change the behavior of a perpetrator without challenging his perception of control, and are quite often used in conjunction with resistance strategies before the use of public strategies. Thus, the findings of the current study suggest that either the participants were responding to escalations in danger, which according to Goodman et al. and Lempert's theory is typically when public strategies are employed, or the participants' safety strategy usage in this study demonstrates action unique to the HIV-IPV phenomenon for which women may be charged to seek greater consultation after recently testing HIV+, or the HIRS protocol did 
spur the participants on to more readily take action for the betterment of their health.

\section{Interpretation of Strategy Utilization}

In summary of safety strategy and safety plan usage, given that data in the current study were procured from individuals undergoing HIV testing services and not from individuals seeking IPV specific services, these numbers compare favorably. Most comparison data comes from samples of individuals who were receiving domestic violence services such as shelters and support groups (Glass et al., 2010; Goodman et al., 2003; Parker \& Gielen, 2014) or from those who specifically sought IPV services (Peltzer et al., 2013). These high numbers demonstrate that those testing HIV+ not only are in need of safety planning strategies but also are motivated to use them as a means to protect themselves. A particularly salient finding from the current study was the popularity of the strategies talking with family/friends and medical professionals, which fall into informal network strategies as well as public strategies. Goodman et al. (2003) explain literature consistently shows women more often begin with private techniques such as placating and resistance strategies and then move into more public strategies. This may underscore a fundamental difference among those who are grappling with the HIV-IPV link versus those who are only navigating the nuances of IPV in their relationship. Or this finding could demonstrate the effectiveness of informing participants about evidence-based practices known to reduce violence during safety planning. 


\section{Feasibility: Domain 4}

The final feasibility domain considered risks involved in study participation. For the item Would it be safe for us to contact you again in 1, 2 or 3 months, there was relatively no difference between dosage levels with $96.3 \%$ of those in Dosage level 1 answering in the affirmative (any contact again was dichotomized post hoc), and $96.2 \%$ of those in Dosage level 2 answering affirmative. Although it took greater effort to reach Dosage level 2 participants for follow-up safety plans and post-tests, these participants seemingly did not mind the extra phone calls as evidenced by the parallel response rates to being contacted again. More, participants' willingness and projected ability to safely correspond again about their relationship difficulties, suggests that it would be plausible (given the resourcing) to extend the follow-up period for a later study. Thus, this finding could be a significant contribution to the rationalization of such a methodological approach in future research.

The items Talking about the relationship difficulties placed you at greater danger and The services you received placed you in greater danger get to the heart of any perceived harm related to study participation. For the item Talking about the relationship difficulties placed you at greater danger, a unanimous number of participants in Dosage level $2(100 \%)$ reported that talking had not placed them in greater danger, and those in Dosage level 1 were similar (96.3\%). For the item The services you received placed you in greater danger, a similar 
percentage of participants in Dosage level $1(95.1 \%)$ reported they had not been placed in greater danger compared to those in Dosage level 2 (97.5\%).

Although these questions show that participants primarily did not find study participation to increase their danger, most studies do not report on perception of increased danger as related to study participation. Instead, this is often operationalized as discomfort, regret, drawback, etc. These items have already been discussed under Acceptability, and response rates in both this Feasibility domain and Acceptability compare positively with other studies. From such comparisons, it can be concluded that the study is feasible to administer without increasing participants' perceived danger. Albeit the numbers were few, future studies should explore how participation and services increased danger among those who reported this was the case.

\section{Violence upon Partner Notification of Serostatus}

To further measure risk among study participants, actual violence experienced was also assessed but, as an HIV-IPV intervention, it was only in relation to partner notification of serostatus. For the item If you notified your partner of your HIV status, did you receive any violence as a result, a significantly higher number of participants in the Standard of Care group (10/81 or 12.35\%) compared to the combined Experimental group (5/160 or 3.13\%) reported having experienced some form of violence upon notifying their partner of their serostatus. Although group differences between dosage levels were non- 
significant, the amount of violence received was further attenuated with the second dosage level.

These percentages compare positively with other studies, but the current study assessed all forms of violence in one item (physical, sexual or emotional), and some studies separated these forms of IPV. One study found $19 \%$ of women had experienced IPV as a result of their HIV status, and slightly higher rates were found in the United States, specifically linking physical violence as a result of a woman's serostatus (Nilo as cited in Hale \& Vazquez, 2011, pg. 18). One source reviewed literature reporting on violence experienced after partner notification in developing countries and found a range between 3.5\%-14.6\% (Medley et al., 2004). Another study found $7 \%$ of participants had reported experiencing IPV before and after serostatus disclosure, 17\% had experienced IPV before their diagnosis, $18 \%$ only after, but $32 \%$ reported experiencing any form of abuse some time before and after they were HIV+ (Gielen et al., 2000). Maman et al. (as cited in Kennedy et al., 2015) found that although violence upon partner notification of serostatus was not significantly lower for their intervention group at a 16-month follow-up, it was significantly lower by a 35-month follow-up. This could indicate that violence related to serostatus disclosure continues to fall, but these findings are not generalizable outside of their study among pregnant women in an antenatal clinic in Durban, South Africa.

This finding of the current study could be the herald of significant progress towards a protocol that effectively mitigates IPV related to serostatus notification among women attending mobile HCT, but results should be contextualized with 
pre-existing knowledge and other relevant considerations must not be ignored. A short review of the history of literature on partner notification assists in determining the ways in which this study contributes to the knowledge base. There is a substantial amount of literature detailing common experiences corollary to partner notification of serostatus. Literature on HIV disclosure spans from the early 90's until recent times, with a large cluster of studies utilizing samples in the United States (Chaudoir, Fisher \& Simoni, 2011) and some in developing countries (Medley et al., 2004). Much of the extant literature does not focus on disclosure experiences specific to those with IPV or those that do are in the United States (Gielen et al. 2000). Disclosure literature using African samples is largely specific to pregnant or postpartum women attending clinics, with the goal of reducing mother to child transmission (Medley et al.; Peltzer \& Mlambo, 2013; Tam, Amzel \& Phelps, 2015)—populations inherently different than those who are not pregnant (though the current study did not ask about pregnancy among participants), experiencing acute IPV or those who are undergoing mobile testing.

Other factors contributing to the current study's findings regarding violence upon serostatus notification will be explored and followed by arguments in support of the study's practical significance. First, the results are flagged by the unequal distribution of those who had previously known their HIV status. More investigation is needed, with a more equally distributed sample set, to confirm these findings; the Standard of Care only had $3(3.66 \%)$ individuals who had been previously diagnosed, and the combined Experimental group had 94 
$(58.75 \%)$ who were past testers. Due to the skewed distribution of the subpopulation, it was not possible to control for tester status because it would have violated statistical assumptions. When comparing new testers only, a higher percentage of those in the Standard of Care group (6/75 or $8 \%$ ) compared to the Combined Experimental group (3/70 or $4 \%$ ) experienced violence upon partner notification, but this no longer remained significant. It cannot be concluded how many of those who had been previously diagnosed went on to inform their partner of their serostatus after study participation.

Second, the current study did not ask how many participants disclosed their serostatus to their partner and did not receive violence, and so it is impossible to fully contextualize how many people in total notified their partner. The item If you notified you partner of your HIV status, did you receive any violence as a result was intentionally engineered so participants were not pressured to disclose if they had notified their partner of their status. Instead, this item along with the item Because of my HIV status I will be in trouble with my partner were to be used as indicators of perceived or actual danger related to a participant's serostatus. By not asking participants to disclose if they notified their partner, the study upheld an ethical standard in tune with the ethical rules of the Health Professions Council of South Africa (Le Roux-Kemp, 2013). Moreover, by not asking participants to disclose if they had notified their partners of their serostatus, the study also circumvented ethical quandaries related to any ensuing HIV criminalization laws given the unfortunate global trend towards criminalizing a positive serostatus (Bernard, Cameron, Hows, \& Mbewe, 2013). 
Third, this study did not ask about all violence experienced after study participation. A possibly relevant postulation is that the older a woman is, the more likely she is to experience physical violence at some point, and therefore her "HIV serostatus becomes less relevant as a determinant of violence" (Maman et al., 2002, pg. 1334). Because IPV is said to inherently escalate, abuse experienced after an HIV+ test may be related to the general cycle of violence but interpreted as a repercussion of serostatus. Another South African study, on pregnant women with IPV who had undergone HIV testing (but were not specifically HIV+) in an antenatal clinic in Mpumalanga province, shows that although Danger Assessment scores dropped by a three-month post-test, $9 \%$ of women experienced an increase in violence and more than $24 \%$ reported physical assault (Matseke et al., 2013). These results cannot be generalized outside of women who are pregnant and clinic-testing in Mpumalanga, but they could indicate the general escalation pattern of IPV or the pattern of escalating violence endured after any testing regardless of one's serostatus.

Conversely, to build a case for how these results do suggest the protocol shows practical significance for safe partner notification strategies, several other findings are elucidated. First, feeding into the last point about the general cycle of violence, Gielen et al. (2000) found that women reported experiencing violence "some time" after serostatus disclosure. Gielen et al. frame this finding within the natural order of IPV as a typically escalating phenomenon. Consequently, abuse experienced after a diagnosis may be not be "instant anger in reaction to the news, but rather some time later either in the context of an ongoing or new cycle 
of violence" (pg. 113). It stands to reason that those who had known their status for some time may be in just as much danger for violence relating to their serostatus as those newly diagnosed as HIV+.

Violence, any time after being diagnosed as HIV+, could increase as the woman begins to rebuild her sense of agency and bravely attempt to receive medical care. Abuse after an HIV diagnosis is said to take on different forms and commonly appears as threats of outing the other's status publically, or withholding medication or the ability to access medical care as needed-all as means to physically or psychologically harm or isolate a woman or conceal abuse (NNEDV, 2014). If IPV is, in fact, escalating, all these tactics specific to the HIV-IPV intersection may act as fodder for more novel ways to harm a woman or maintain power over her.

Second, considering significantly more participants in the Experimental group employed a consultation strategy to mitigate IPV, it is consistent with the literature that this would lead to less general violence. Goodman et al. (2003) assert that the use of public safety strategies, such as contacting friends/family or medical and mental health professionals, act as stronger forces to prevent violence. This is not a perfect comparison as Goodman et al. are comparing violence received after the use of these public strategies versus private strategies like placation and resistance. However, given the greater usage rate of public consultation strategies found among Experimental participants, it could be deduced that this played some role in reducing violence, in general, for those in 
the Experimental group, but it cannot be specifically connected to the violence experienced upon partner notification for the aforementioned reasons.

Third, while it may first be assumptive that those who had previously been diagnosed as HIV+ would not experience parallel risks upon partner notification or even have the same numbers who had yet to notify their partners, a shocking number of participants from all groups reported a sharp increase in fear related to getting to medical appointments between pre-test and post-test. For the item, You feel safe getting to medical appointments, $(236 / 248$ or $95.16 \%)$ of the total sample reported at pre-test they currently felt safe getting to medical appointments, but by post-test this percentage had almost reversed so that only (102/248 or $41.13 \%)$ answered yes to I have felt safe getting to medical appointments. This means that at pre-test only $4.84 \%$ anticipated they would feel unsafe getting to medical appointments, but within the 30 days after testing $58.87 \%$ reported no longer feeling safe getting to medical appointments. It is a considerably alarming finding that perceived risk grew for over half of the sample within the first 30 days after testing, independent of tester status. And most salient to the present discussion were the significantly diminished percentages of those feeling safe getting to medical appointments among past testers (those previously diagnosed as HIV+). Among past testers, (96/97 or $98.97 \%)$ reported they felt safe at pre-test, and only (35/97 or $36.08 \%$ ) reported feeling safe by post-test. Although new testers' feelings of safety in getting to medical appointments also diminished, falling from 140/151 (92.72\%) at pre-test to $(82 / 149$ or $55.03 \%)$ at post-test, the most dramatic change occurred among the 
past testers who had overall $62.89 \%$ report a change in feelings of safety compared to the new testers at $37.69 \%$. This demonstrates it cannot be assumed that the past tester sub-population, those previously diagnosed as HIV+, did not also experience grave difficulties getting to medical appointments despite these participants having already been aware of their own serostatus. The on-going danger associated with HIV serostatus that was observed in this study is consistent with the assertion of Medley et al. (2004) that the disclosure process (partner notification) is often not immediate but occurs over time with an increasing amount of disclosure occurring over time. Further, Wong et al. (2009) found that among a general sample of men and women (not specific to IPV) those who had already disclosed their serostatus to their sexual partners had known their serostatus 7 months longer than those who had not disclosed. Although literature consistently finds that most women disclose their serostatus to their partner (Gielen et al., 2000) and do so for various reasons (Peltzer \& Mlambo, 2013), a systematic review showed individuals are more apt to tell someone they have a close intimate relationship with (Chaudoir et al., 2011), with disclosure among pregnant women being strongest for those who had strong partnership with no history of domestic violence (Tam et al., 2015). Thus, it cannot be assumed that any of those who had previously been diagnosed had disclosed their status to their partner, and even for those with new diagnoses, it cannot be assumed how many had disclosed to their partner.

The fourth reason these results could be practically significant considers the potential role of baseline danger scores at pre-test. While no literature could 
be found on predicting danger associated with the HIV-IPV link, the literature that exists on predicting IPV-only risks suggests the best model for predicting IPV is use of Campbell's Danger Assessment (DA) in conjunction with a woman's reported perception of risk (Roehl, O'Sullivan, \& Webster, 2005). If the HIRS scale measures perception of risk, its use in conjunction with the DA could be the strongest indication into actual danger levels for those affected by the HIV-IPV link. Although pre-test DA scores for the combined Experimental group were not significantly higher than the Standard of Care group, scores for the Danger Indicator (whole pre-test, including danger associated with the HIV-IPV intersection) showed that those in the Standard of Care group scored significantly lower at pre-test than the combined Experimental group. According to this, those in the combined Experimental group would be predicted to have a slightly higher occurrence of re-assault. Further, those in the Standard of Care group reported a significantly lower baseline level of physical and sexual violence at pre-test than the combined Experimental group. In general, women who have endured IPV report "higher personal-risk ratings for future violence than women without such experience" (Helweg-Larsen as cited in Harding \& Helweg-Larsen, 2008, pg. 9). One interpretation of these findings is that because the current study did not have baseline equivalence for the two variables of the Danger Indicator score and reports of physical and sexual abuse, the intervention was possibly more effective than the non-significant results indicate.

The fifth, and final, reason these results could be practically significant builds on the aforementioned reasons and more closely inspects baseline 
perceptions of danger related to serostatus as a mechanism for including women's perceived risk in tandem with an actuarial score for risk. Results from the item You will be in trouble because of your HIV status demonstrate that, aside from containing more participants who had been previously diagnosed as $\mathrm{HIV}+$, those in the combined Experimental group had greater anticipation at pretest that their serostatus could be dangerous in their relationship. About $31 \%$ of the combined Experimental group answered yes, compared to only $29.4 \%$ answering yes in the Standard of Care group. For the combined Experimental group, percentages were slightly higher for new testers (34.29\%) than past testers $(28.88 \%)$. However, when controlling for this item, the difference between violence upon partner notification of serostatus remained significantly higher among those who reported they would not be in trouble with their partner in the Standard of Care compared to those in the combined Experimental group.

Cattaneo et al. (2007) define situations where individuals predict they are low risk for re-abuse but go on to experience re-abuse as false negatives, and those who predict they are high risk but then do not experience re-abuse as false positives. This source argues there would be qualitative differences between false positive and false negative groups with those with more severe violence who also "saw themselves at low risk...[being] almost two times more likely to be incorrect than correct," (pg. 438). Unfortunately, this study did not assess for severity of physical abuse, and group comparisons on severity cannot be drawn. Yet, those in the combined Experimental group had significantly more participants reporting any physical abuse at pre-test, and this could suggest they 
would be more likely to predict re-abuse but went on to experience less re-abuse relating to partner notification despite the prediction they would be in trouble with their partner because of their serostatus. Hence, this finding is consistent with the Harding \& Helweg-Larsen (2008) assertion that those who perceive themselves to be in greater risk for danger have been shown to have greater intentions of making protective changes in their relationship.

Given that those in the Experimental group had more physical abuse, increasing their likelihood to take action to protect themselves, it can be hypothesized that receipt of the experimental components of HIRS protocol acted as the stimuli inciting participants to make changes by way of first shifting their normative beliefs about abuse and then exposing them to available resources. One of the central components of the HIRS protocol is the presentation of danger levels (from the Danger Indicator) to participants. This is done in the beginning of safety planning to inform participants of danger levels in their relationships. It is possible that because those in the Standard of Care group were not informed of their danger levels they were less aware of the potential risks in their relationships and less prepared for re-assault that could ensue upon partner notification of serostatus. Another central component of the HIRS protocolparticipants in the experimental group were informed about potential danger with partner notification and asked if they wanted to develop a plan to safely notify their partners. As a result, the item Because of my HIV status I will be in trouble with my partner may be a better indicator of group comparisons rather than tester status. As referenced above, this item was also found to be a significant indicator 
for protocol effectiveness with linkage to care, as more people answering yes to the item among the combined Experimental group (50\%) linked to care compared to those answering yes and linking to care in the Standard of Care group (23.1\%)—also consistent with the assertion of Harding \& Helweg-Larsen and ground in Theory of Planned Behavior.

\subsection{Strengths}

Though this study had many strengths, six primary areas of strengths are enumerated. First, rigorous methodology was maintained while also abiding by the ethical standards laid forward by the World Health Organization for Ethical and Safety Recommendations for Research on Domestic Violence Against Women. Using randomized group allocation, the study assigned participants into a Standard of Care group and an Experimental group that contained two treatment arms to test variations in outcomes by protocol dosage levels. Sample size for group allocation was based on an a priori power analysis and the amount needed was exceeded. The Standard of Care group was offered first-line support in accordance with ethical standards, and only a small number of those utilized resources on their own. These contaminating cases were extracted from appropriate analyses as distinguished within Chapter Four.

Second, almost all participants were retained for post-test data collection. This is an extremely high level of retention and shows great evidence for the feasibility of the protocol. Of the 255 who participated in pre-test data collection, $249(97.65 \%)$ of those were retained for post-test data collection, and $99.97 \%$ 
were reachable with only a small amount opting out of post-test collection despite being reachable. This is a remarkable retention rate compared to other studies. Matseke et al. (2013) found a retention rate in an IPV study in South Africa of $52.5 \%$ with only a slightly longer follow-up period of 3-months; Peltzer et al. (2013) used a 6-month follow-up period with IPV survivors and only had a retention rate of $44 \%$. If one study's use of low survey completion rates was correctly interpreted to indicate harm experienced by participants (Appollis et al. 2015), the current study bodes well in feasibly administering a study over a sensitive subject with little to no harm as a corollary.

Third, the study included not only participants who had experienced physical and sexual abuse but also those with psychological abuse (non-violent control) in their current relationship. Rees et al. (2014) point out that this is often not addressed by studies analyzing IPV prevalence, "although it appears to be common and has serious mental health implications" (pg. 2). By including those with psychological abuse, this form of abuse is upheld as a commensurate threat against human rights (as physical and sexual abuse)—allowing those with psychological abuse to also reap study benefits, and new knowledge was generated on psychological abuse and HIV.

Fourth, the study addresses a major gap in knowledge on the HIV-IPV intersection, having tested and found to be appropriate two new psychometric tools. One tool was the HIV IPV Risk and Safety (HIRS) scale. This scale is a completely new tool designed specifically to address risk associated with the HIV-IPV intersection. The other instrument created and tested within this study 
was the Danger Indicator. This tool includes the HIRS scale within it but is also comprised of several other scales with strong empirical support for their validity and reliability of use in South Africa. Both were shown to be appropriate instruments insofar as the Danger Indicator was sufficiently unidimensional to be used as a global score for danger prediction, and the HIRS scale was shown to have very good reliability. Although both instruments need some further refinement (see Limitations), the preliminary findings on them show they represent a significant contribution to measuring perceived risk and safety associated with HIV-IPV intersection.

Not only do the results of this study add to the IPV knowledge base by further testing the use of safety strategies among IPV victims-where there is a paucity of information—but the results also help establish a $7^{\text {th }}$ domain for IPV safety strategies that is specific to those living with both HIV and IPV. The final way this study addresses a knowledge gap is by demonstrating what happens within the first 30 days after testing HIV+ during mobile testing. Trends in linkage to care, immediately following HIV diagnosis, were previously unknown for those experiencing IPV. Some information had been gathered on abuse after testing but those were for pregnant women, not necessarily testing $\mathrm{HIV}+$, and in a different province at an antenatal clinic. This study's results help gain insight into the volume of IPV prevalence among mobile testers in Gauteng province and what occurs immediately following diagnosis. Also shown is the heightened sense of danger, related to serostatus, present among those previously diagnosed. 
Fifth, study results contribute substantially to the field of knowledge on the HIV-IPV intersection. Items were included in the study to measure not only effectiveness of the protocol but also feasibility and acceptability, which are integral in determining the risk/benefit ratio-an ethical necessity when conducting research on sensitive subjects (Appollis et al., 2015). These findings demonstrate how an HIV-IPV protocol can be effective and well received while inflicting very little risks, building greater knowledge on the cost/benefit ratio of research of the sensitive subject of abuse. Findings also present a protocol promising in promoting linkage to care for those aged 33-43 and mitigating violence upon partner notification of serostatus.

The sixth and final strength of this study was its ability to accomplish all strengths hitherto listed while employing a protocol shown to be brief in administration. The importance of this finding cannot be overemphasized. Sources call for more efficient approaches in developing countries (UNAIDS, 2013a), and adding a needed IPV component cannot become a burden to already-existing and vital HCT services. Brevity is key to feasibility given the extreme financial and time constraints experienced in such a low resource, high prevalence area as Gauteng province, South Africa. Therefore, this protocol shows great promise for the feasibility of integrating an IPV protocol into mobile HCT as a means to mitigate IPV and safely promote linkage to care.

\subsection{Limitations}


As all studies have limitations, and, so too, did this study. Despite the randomization process, there was not group equivalency on a few variables. Groups differed by portion of physical and sexual abuse reported at baseline as well as pre-test scores for Danger Indicator levels. The unequal distribution of those newly diagnosed versus previously diagnosed (tester status) was the limitation causing the most hindrance to the process of garnering the practical significance of study results. While the study exceeded the required sample size determined necessary through power analysis, the unequal distribution of those previously diagnosed prohibited the use of even elementary analytical methods. Although their inclusion helped generate new information about the on-going role of perceived danger related to serostatus for those previously diagnosed as HIV+, caution should be used in interpreting the finding that the Standard of Care group experienced significantly more re-assault upon partner notification of serostatus and this research question should be further explored in future studies with equally distributed new testers to confirm these results.

While the vast majority of participants received the protocol within 30 days from HIV testing, a small percentage received the intervention slightly over 30 days. It took some participants longer than others to be reached for each component of the study, and this was inherent to the telephonic medium. Although it could have been an important confounder if the time to reach extended well beyond 30 days, this was not the case in this study.

The research team administering this study was not blinded to study group allocation because the research team had to administer the varying treatments 
based on group allocation. However, participants were blinded to group allocation. Although the randomization process was rigorous, the lack of blinding among the research team could be a threat to the validity of the study.

Limited information was collected on participant demographics. Most other studies collect information on participants' education levels and other data that has been shown to be mediate HIV and IPV risk factors. Such information would have allowed for stronger contextualization of the data and more robust interpretations of the study's practical significance. Along the same lines, the study did not address some of the more common elements found in similar studies such as trauma-related co-morbidity among survivors (Appollis et al., 2015) or the role stigma plays in the HIV-IPV intersection (Zembe et al., 2015). As discussed previously, the study did not ask about all abuse endured after participation but merely focused on abuse related to partner notification of serostatus. All such material could have allowed for stronger contextualization of results. Yet, most of these prospective items were jettisoned as an effort to maintain brevity, and because there is already a substantial body of information in existence on many of the demographic correlates of IPV in areas with high HIV prevalence (Abrahams, Mathew, Martin, Lombard \& Jewkes, 2012; Abramsky et al., 2011; Adams et al., 2011; Dunkle et al., 2004; Jewkes et al., 2002). However, it would be advantageous for future studies to include more demographic information to draw further parallels with other studies and to uncover any other trends that could exist relative to the HIV-IPV intersection, as such information 
could demonstrate the effectiveness of the current protocol on various populations.

There are other forms of IPV not considered in this study, i.e. IPV in samesex relationships, abuse perpetrated by women against men, or IPV in the form of financial abuse. These topics should also be explored in research on the HIVIPV link. For example, the South African Domestic Violence Act includes all types of IPV discussed here but also includes economic abuse, stalking, property damage and the act of illegally entering someone's residence (Peltzer et al., 2013). While this study did not exclude those in same-sex relationships, it did not ask participants about their sexual orientation and operated from the theoretical assumption that IPV is largely gender-based and perpetrated by men against women. Other studies should include other forms of IPV and allow for the inclusion of male victims.

After the study's implementation, new information surfaced from personal contact with authors of two of the sub-scales used within the study. Personal communication (J. Campbell, personal communication, July 29, 2015) with the creator of the Danger Assessment led to a slight change in the danger values assigned to a few different items within this sub-scale. Changes were made to this portion of the Danger Indicator and participants were updated on this information at the next contact (some at follow-up safety planning and others post-tests). However, this only affected a small portion of the group as this change occurred only a few weeks into study recruitment when recruitment was at its slowest (due to poor initial buy-in from those administering HCT and 
responsible for study recruitment). Ultimately, these minor changes only altered the overall Danger Indicator score by 1 point, causing it to go from 50 to 49 total points, which through inspection was not shown to have changed any participant's overall danger level (in 5 point increments). Hence, the integrity of the intervention was not altered due to this change.

Contact with M.P. Johnson (personal communication, July 23, 2015), one of the authors of the non-violent control scale, provided new information relevant to the methods used to measure IPV, particularly with the non-violent control scale. The dichotomous items used to assess non-violent control were based on Johnson \& Leone's (2005) manuscript, but through personal email exchange and the sharing of manuscripts unpublished at the time of this study's implementation, a new method was realized. This manuscript by Hardesty et al. (2015) was published 1.5 months into this study's implementation, and thus changes were not possible. Hardesty et al. negate the use of measuring IPV by merely tallying IPV tactics experienced and explain that, instead, IPV must be considered in terms of frequency and severity which can only be obtained through asking about the complexity of each tactic used rather than the amount of different tactics used. This information is mostly only relevant to the process of determining IPV typology among a sample set, which the present study does not attempt to do (see recommendations for future research), but this is important because the dynamics of the IPV experienced can be drastically different from one typology to another (see implications for future practice). Such a methodological approach would have enriched the data in the present study, but 
doing so would have certainly jeopardized the brevity for which the protocol was administered.

Finally, while the HIRS scale and Danger Indicator both represent significant contributions to the HIV-IPV knowledge base, both come with limitations. Though the HIRS scale was shown to have strong reliability (Cronbach alpha .882), the validity of the scale (the mean of corrected item total correlation was .364) was problematic (see recommendations for future studies), and, thus, results for the scale should be interpreted with caution. Additionally, the Danger Indicator, while shown to be sufficiently unidimensional, had only $19 \%$ of variance accounted for in the final fit during Exploratory Factor Analysis (see recommendations for future studies). More, the validity and reliability of these psychometric instruments were specific to the validation sample and future studies should reassess validation for different populations.

\subsection{Implications for future practice}

Literature highlights the urgent need for interventions that address the HIV-IPV link; interventions are needed to improve access and retention to healthcare services and promote strategies for safe partner notification of serostatus. Each component of the HIRS protocol was woven into the protocol because it was grounded in the literature as an empirically supported practice in the areas of either mobile HCT or intimate partner violence. This study presents

findings from a first effort in creating a protocol that effectively mitigates IPV as a means to promote health and safety after testing HIV+ in mobile HCT. While the 
results of this study show early promise for an acceptable and feasible protocol that has effective strategies to reduce assault upon partner notification of serostatus and promote safe linkage to care among those aged 33-43 years, further testing and refinement of the HIRS protocol is needed before fully implementing the protocol as a standard of care.

Further testing is required, before implementing the protocol into practice, to clarify the current results and strengthen or challenge findings on effectiveness. From what is known about the protocol, several challenges may emerge with its use as a standard of care. One the one hand, the telephonic nature of the protocol would facilitate administration by the NGO's current links to care call centre program. On the other hand, some logistical challenges would need to be circumvented. More ad hoc funding would be required so that staff time and monies are not detracted from current HCT services. Additionally, all staff would need on-going training on the HIV-IPV link and ways to provide supportive and empathic services to IPV victims. Social workers are poised as the best candidates for such a role, as they are trained to administer clinical services from a place of non-judgment and empathy while applying knowledge of the person-in-environment. One way to parcel out protocol administration time would be to offer the Danger Indicator during HCT but prior to testing uptake. Those who meet IPV eligibility would then receive safety planning during their contact with the links to care call centre program. However, administration of the Danger Indicator prior to testing may have challenges as well; this could reduce the numbers of those wanting to test if it took too much of participant's time, and 
screening all participants would be costly in either staff time or resources (the latter would be the case if the screening were to be administered through interactive media).

Additionally, the protocol itself should be refined to address the varying types of IPV, as each IPV typology requires different intervention modalities. Whereas the typology of Intimate Terrorism typically includes the use of systematic power and control and has been shown to escalate in severity or frequency over time, another typology of Situational Couple Violence can present with gender parity and is said to not be maintained by power and control but instead by maladaptive coping skills employed when the demands of stress exceed resources.

Finally, other populations may benefit from the HIRS protocol. As cited, women report experiencing IPV after an HIV+ diagnosis, but some of those have not experienced IPV before the diagnosis. Thus, only assisting those who meet IPV eligibility may be marginalizing groups of individuals who are vulnerable. Consequently, all women may benefit from receipt of the HIRS as a means for general safety planning after a diagnosis. More, services should be extended to men; they also experience IPV victimization, and there is a great need for protocols effective with batterers. Given the ample literature citing the need for IPV services with men (Boonzaier \& Gordon, 2015; Jewkes et al., 2011), funders would likely find such an initiative compelling and grant-worthy.

\subsection{Recommendations for future research}


Despite the call for more evidence-based methods addressing the HIVIPV intersection, there is still a paucity of information on effective ways to mitigate deleterious health outcomes among women diagnosed as HIV+. Greater attention is needed on women already living with HIV to help reduce barriers to care and assault related to a seropositive status. HCT has been shown to be a prime way to reach women affected by the HIV-IPV link, and more research is needed to refine best practices for assisting this population post diagnosis.

Future studies utilizing similar populations should include some components of the HIRS protocol given that it was overwhelmingly well received among all participants in this study. Because the experimental components were also found to be helpful and safe to administer, future studies could use the safety plan in this study but should continue to enhance it with current research as such knowledge unfolds. The high retention rate in this study, coupled with the number of participants who reported it would be safe to be contacted again at the 1, 2 and 3-month marks, suggests a future study could also extend the follow-up period to gain a more accurate depiction if participant experiences fluctuate before and after the first 30 days of a diagnosis or testing.

Findings from this study will inform future studies in various ways. Namely, the HIRS protocol may be refined in a Phase III clinical trial in order to strengthen its suitability for practice. Such a study would allow for a larger sample size, enabling the covariate of tester status (those previously diagnosed), and more administration time to glean richer data over IPV typology, frequency and 
severity, using continuous variables permitting the use of more sophisticated analytical methods and subsequently more robust practical significance.

Given that the current protocol was found to be safe, acceptable and feasible to implement, further effectiveness testing could be done with more rigor by implementing an amended HIRS protocol that masks group allocation from those administering the instruments. Future studies could do this by changing the instruments to be identical so that a designated person would only administer the pre and post-test instruments while being blinded to participant group allocation.

It is recommended that the standard HIRS protocol adopt the second dosage level, as outcomes were strengthened by the second dosage. However, should financial and time limitations present as insurmountable barriers, effectiveness does not appear to hinge on the second dosage level. The extra safety plan was found to be beneficial in regards to minor increases in acceptability and satisfaction, increases in safety plan and safety strategy usage and the attenuation of re-assault related to partner notification of serostatus. The follow-up safety plan was, however, found to be cumbersome in administration given the excessive amount of attempts it took to reach participants for this component.

Future studies should be designed to more clearly assess for the effectiveness of safe partner notification strategies. A systematic review of safe partner notification strategies was published after this study began, but it contains important recommendations for future research about strategies to mitigate violence upon partner notification. Essentially, this source argues that 
despite the global charge for more interventions addressing safe partner disclosure, very limited research has focused on rigorously demonstrating the effectiveness of notification strategies (Kennedy et al., 2015). Future studies should ask more explicit (but ethically crafted) questions to assess who has already disclosed their status to their partners, each type of violence (physical, sexual and emotional) should be separately assessed, and study designs should include longer follow-up periods to enhance comparability with other studies reporting on this topic. The findings of the current study demonstrate the need for greater attention to be given to those who are previously diagnosed but remain fearful, as they too experienced heightened fear in the 30 days after undergoing mobile HCT. A greater understanding of what motivated this group to return for CD4 cell testing would assist with more knowledge on this sub-population. For those who had already disclosed their serostatus to their partner, important information may be gleaned on effective strategies for safe partner notification.

Other sub-populations should be considered to improve the comparability of study findings or to assist those commonly neglected by current research. Future studies should assess for all violence experienced between pre and posttest collection and include group comparisons in linkage rates between those with IPV and without IPV. Linkage rate comparisons would be enhanced if studies of the HIV-IPV intersection also tracked participants' CD4 cell counts. Fluctuations in CD4 cell counts could demonstrate changes in physical health among participants, facilitate comparability with other linkage research given that many use CD4 stratum to interpret linkage rate success, and help suss out 
motivators for linkage to care among those meeting ART eligibility. More information is needed on participants aged $33-43$ to learn what factors may have allowed greater rate of linkage to care among them. Additionally, there is a great need to include males in studies on HIV and IPV, with effective batterer interventions needed as well as programs shown to be effective with male victims of IPV. A greater focus should also be placed on exploring the HIV-IPV intersection in same-sex relationships in South Africa.

To determine if the HIRS protocol is detecting increases in risk or heightened awareness of IPV dynamics, future studies should include a means for assessing increases in knowledge of IPV. This, in conjunction with assessing for all violence experienced between pre and post-test, will shed greater light on fluctuations in risk and safety among participants between study groups.

Safety strategies in the current study were assessed using combined categories. For example, the item on legal strategies lumped together calling the police, talking to a lawyer, and taking out an order of protection. Each of these could have different implications, and future studies could separate these out to better assess which were most utilized and helpful. Further, the helpfulness of each strategy should be considered as well as how perceptions of helpfulness vary among those with higher danger scores (as found in Goodman et al., 2003), and the use of a likert scale would allow for comparison with germane literature which report mean scores for helpfulness.

As united efforts are needed to continue fighting the insidious interlinked epidemics of HIV and IPV, there is a great need for cross-cultural exploration of 
how IPV differs throughout various international contexts. For example, the role that concepts such as love, hope and dreams play in IPV relationships among people living with HIV should be further explored as these concepts are suspiciously omitted from literature on IPV in South Africa, even among qualitative findings.

Greater synergy is needed between those creating protocols such as the HIRS and the communities they are administered in so that community-level change may happen alongside micro level change. For example, the four most popular strategies employed from the safety plans were contact with family/friends, medical professionals, mental health professionals and clergy. Each of these areas as a sole help line raises concern given that doctors were found to be grossly under-trained, mental health professionals may not operate from a strength-based or empowerment model, clergy were burned out with their role in helping survivors and family and friends likely have adopted similar cultural beliefs of normalized gender inequalities. For such a goal, initiatives such as the HIRS, targeting the HIV-IPV intersection, will likely need to work more closely with IPV-specific programs to create a more substantial break in the massive flow of gender inequalities amassed in South African culture.

\subsection{Conclusion}

Eradication of the HIVIAIDS epidemic hinges on the global response to interconnected human rights' issues such as gender-based violence and its alltoo-common manifestation of intimate partner violence. South African women 
have been hit the hardest by both HIV and IPV, and sources have called for more rigorously tested approaches that jointly target these phenomena as a means to mitigate future assault related to a seropositive status and reduce barriers to HIV care. Findings from the study Mitigating Intimate Partner Violence to Promote Safe Linkage to Care Among South African Women Testing HIV+ During Mobile Counseling and Testing show positive results of a promising protocol developed to mitigate violence related to the HIV-IPV intersection while promoting safe HIV outcomes.

The study furthers the HIV-IPV knowledge base by testing two new psychometric instruments and showing the feasibility and acceptability of a new HIV-IPV protocol. The psychometric instruments of the Danger Indicator and the HIV IPV Risk and Safety (HIRS) scale were shown to be suitable for use with the HIRS having good reliability and the Danger Indicator having the latent variable of physical assault. However, both instruments will need some refinement to improve their validity in future studies. All of the study's aims were achieved: The HIV IPV Risk and Safety (HIRS) protocol was found to be feasible to implement, as evidenced by extremely high retention, brevity in administration, and high rates of safety strategy usage with minimal risks experienced as a result of participation. Participants reported high levels of satisfaction, as evidenced by an overwhelming number of positive ratings, and the Standard of Care group contained minimal contamination with very few participants in this group receiving services elsewhere. 
The HIRS protocol was shown to be effective with regards to mitigating violence upon partner notification of serostatus and promoting greater linkage to care for those in the experimental group but significantly more so among those aged 33-43 years. Moreover, the protocol achieved effectiveness in these areas while also demonstrating that the perceived benefit of study participation far outweighed reported harms or risks.

New trends were uncovered in how South African women enduring IPV experience difficulties related to a seropositive status and the various strategies utilized for self-protection. This study evinces the experiences of women with IPV in the first 30 days following an HIV+ diagnosis: across all groups, significantly more people reported decreased safety in getting to medical appointments, indicating danger associated with getting to medical appointments increases within the first 30 days after a diagnosis. More, a surprisingly large number of women previously diagnosed as HIV+ reported being fearful their serostatus would cause them to be in trouble in their relationship, showing that danger associated with serostatus remains a concern for women and may also be worse some time after diagnosis.

Women who received the HIRS protocol were shown to be self-determined to take action for self-protection while suffering significantly less re-assault upon partner notification than those who did not receive the protocol. Most of the experimental group deployed at least one safety strategy, and consistent with the literature, the most popularly utilized strategies (consulting with a friend/family member or medical professional) have been shown to reduce the likelihood of re- 
assault. Conversely, the least utilized strategies (placation and resistance) have been shown in the literature to increase the likelihood of re-assault. The large number of participants deploying safety strategies to effectively protect themselves reveals the influx of individuals undergoing HCT daily who could benefit from the integration of IPV assistance into mobile HCT; this is especially true considering significantly fewer participants in the Standard Of Care group consulted with someone about IPV compared to those in the Experimental group. The number of participants utilizing public safety strategies shows the effectiveness of a brief psychoeducational intervention in galvanizing South African women to take action to protect themselves from IPV through the reclaiming of power rather than acts of placation or complicity.

Overall, most of the linkage rates for all groups and ages were lower than those found in extant literature, especially among those aged $\geq 23$ years; thus, the need for greater efforts in mitigating IPV as a barrier to HIV care is demonstrated. The HIRS protocol was shown to be safe, feasible, acceptable and effective. Its effectiveness in facilitating safe partner notification of serostatus could be a significant contribution to the knowledge base, but more research is needed to confirm these results. 


\section{REFERENCES}

References marked with an asterisk indicate studies included in meta-analyses. Abdool Karim, Q., Meyer-Weitz, A., Mboyi, L., Carrara, H., Mahlase, G., Frohlich, A., \& Abdool Karim, S.S. (2008). The influence of AIDS stigma and discrimination and social cohesion on HIV testing and willingness to disclose HIV in rural KwaZulu-Natal, South Africa, Global Public Health, 3(4), 351-365. doi: 10.1080/17441690802076496.

Abrahams, N., Mathew, S., Martin, L.J., Lombard \& Jewkes, R. (2012). Intimate partner femicide in South Africa in 1999 and 2009. PLoS Medicine, 10(4), e1001412. doi: 10.1371/journal.pmed.1001412.

Abramsky, T., Devries, K., Kiss, L., Francisco, L., Nakuti, J., Musuya, T., Kyegombe, N., Starmann, E., Kaye, D., Michau, L., Watts, C. (2012). A community mobilisation intervention to prevent violence against women and reduce HIVIAIDS risk in Kampala, Uganda (the SASA! Study): Study protocol for a cluster randomized control trial. Bio Med Central, 13(96), 122. http://www.trialsjournal.com/content/13/1/96.

Abramsky, T., Watts, C.H., Garcia-Moreno, C., Devries, K., Kiss, L., Ellsberg, M., Jansen, H.AFM., Heise, L. (2011). What factors are associated with recent intimate partner violence? Findings from the WHO multi-country study on women's health and domestic violence. BMC Public Health, 11, 109-126. https://www.biomedcentral.com/1471-2458/11/109. 
Adams, J.L., Hansen, N.B., Fox, A.M., Taylor, B.B., van Rensburg, M.J., Mohlahlane, R., Sikkema, K.J. (2011). Correlates of HIV testing among abused women in South Africa. Violence Against Women, 17(8), 10141023. Doi: $10.1177 / 1077801211414166$.

Ahmad, F., Hogg-Johnson, S., Stewart, D.E., Skinner, H.A., Glazier, R.H., Levinson, W. (2009). Computer assisted screening for intimate partner violence and control. Annals of Internal Medicine, 151, 93-102.

AIDS.gov (2015). HIV Care Continuum. Retrieved on February 6, 2016 from https://www.aids.gov/federal-resources/policies/care-continuum/

Ajzen, I. (2006). Behavioral interventions based on the theory of planned behavior. Retrieved from https://people.umass.edu/aizen/pdf/tpb.intervention.pdf

Appollis, T.M., Lund, C., de Vries, P.J., \& Mathews, C. (2015). Adolescents' and adults' experiences of being surveyed about violence and abuse: A systematic review of harms, benefits, and regrets. American Journal of Public Health, 105(2), e31-e45.

AVERT (2015). Women and HIV. Retrieved from https://www.avert.org/node/387/pdf

Baig, A.A., Ryan, G.W., Rodriguez, M.A. (2009). Provider barriers and facilitators to screening for intimate partner violence in Bogota, Colombia. Health Care for Women International, 33, 250-261. doi: 10.1080/07399332.2011.646368. 
Ball, H. (2013). Intimate partner violence linked to HIV infection among Ugandan women. International Perspectives on Sexual and Reproductive Health, 39(3), 165-166.

Bassett, I.V., Regan, S., Chetty, S., Giddy, J., Uhler, L.M., Holst, H., Ross, D., Katz, J.N., Walensky, R.P., Freedburg, K.A., Losina, E. (2010). Who starts antiretroviral therapy in Durban, South Africa?... not everyone who should. AIDS, 24(1), S37-S44.

Bassett, I.V., Regan, S., Luthuli, P., Mbonambi, H., Bearnot, B., Pendleton, A., Robine, M., Mukuvisi, D., Thulare, H., Walensky, R.P., Freedberg, K.A., Losina, E., Mhlongo, B. (2014). Linkage to care following communitybased mobile HIV testing compared with clinic-based testing in Umlazi Township, Durban, South Africa, HIV Medicine, 15(6), 367-372. doi: 10.1111/hiv. 12115 .

Bassett, I. V., Govindassamy, D., Erlwanger, A. S., Hyle, E. P., Krazner, K., Van Schaik, N., Noubary, F., Paltiel, A.D., Wood, R., Walensky, R.P., Losina, E., Bekker, L-G., Freedberg, K. A. (2014). Mobile HIV screening in Cape Town, South Africa: Clinical impact, cost, and cost-effectiveness. PLoS One, 9(1). doi: 10.1371/journal.pone.0085197.

Beer, L., Fagan, J., Garland, P., Valverde, E.E., Bolden, B., Brady, K., Courogen, M., Hillman D., Neaigus, A., Bertolli, J. (2012). Medication-related barriers to entering HIV care. AIDS Patient Care and STDs, 26(4), 214-21. DOI: 10.1089/apc.2011.0407. 
Bendall, C. (2010). The domestic violence epidemic in South Africa: Legal and practical remedies. Women's Studies, 39, 100-118. doi:

$10.1080 / 00497870903459275$.

Bennett, L., Riger, S., Schewe, P., Howard, A., Wasco, S. (2004). Effectiveness of hotline, advocacy, counseling, and shelter services for victims of domestic violence. Journal of Interpersonal Violence, 19(7), 815-829. Doi: $10.1177 / 0886260504265687$.

Bernard, E.J., Cameron, S., Hows, J, Mbewe, S. (2013). Advancing HIV justice: achievements and challenges in global advocacy against HIV criminalisation. Taken from a progress report on achievements and challenges in global advocacy against HIV criminalisation. HIV Justice Network, Global Network of People Living with HIV and MANET. Retrieved from http://www.scribd.com/doc/190880968/Advancing-HIVJustice-Achievements-and-challenges-in-global-advocacy-against-HIVcriminalisation-African-update-ICASA-2013.

Bloom, T.L., Glass, N.E., Case, J., Wright, C., Nolte, K., Parsons, L. (2014). Feasibility of an online safety planning intervention for rural and urban pregnant abused women. Walters Kluwer Health/Lippincott Williams \& Wilkins, 63(4), 243-251. Doi: 10.1097/NNR.0000000000000036.

Boonzaier, F.A. \& Gordon, S.F. (2015). Responding to men's violence against women partners in post-apartheid South Africa: On the necessity of identification across identity's intersection. British Journal of Criminology, 55, 1096-1114. doi: 10.1093/bjc/azv090. 
Bowen, E. (2011). An overview of partner violence risk assessment and the potential role of female victim risk appraisals. Aggression and Violent Behavior, 16, 214-226. doi: 10.1016/j.avb.2011.02.007.

Bowen, D.J., Kreuter, M., Spring, B., Cofta-Woerpel, L., Linnan, L., Weiner, D., Bakken, S., Kaplan, C.P., Squiers, L., Fabrizio, C. \& Fernandez, M. (2009). How we design feasibility studies. Am J Prev Med., 36(5), 452457. doi: 10.1016/j.amepre.2009.02.002.

Bundy, C. (2004). Changing behavior: using motivational interviewing techniques. Journal of the Royal Society of Medicine, 44(97), 43-48.

Burke, J.G., Denison, J.A., Gielen, A.C., MacDonnell, K.A., O'Campo, P.A. (2004). Ending intimate partner violence: An application of the transtheoretical model, AM J Health Behav., 28(2), 122-133.

Campbell, J.C. (2004). Helping women understand their risks in situations of intimate partner violence. Journal of Interpersonal Violence, 19(12), 14641477. doi: $10.1177 / 0886260504269698$.

Campbell, J.C., Webster, D.W., Glass, N. (2009). The danger assessment: validation of a lethality risk assessment instrument for intimate partner femicide. Journal of Interpersonal Violence, 24(4), 653-674. doi: 10.1177/0886260508317180.

Campbell, J.C., Webster, D.W., Kozial-McClain, J., Block, C.R., Campbell, D., Curry, M.A., Gary, F., McFarlene, J., Sachs, C., Sharps, P., Ulrich, Y., Wilt, S.A. (n.d.). Assessing risk factors for intimate partner homicide. NIJ Journal, 250, 15-19. 
Cardey, S. Garforth, C., Govender, C., and Dyll-Myklebust, L. (2013).

Entertainment education theory and practice in HIV/AIDS communication:

A South Africa/United Kingdom comparison. Critical Arts Project and Unisa Press. Critical Arts: A South-North Journal of Cultural \& Media Studies, 27(3), Online 1992-6049, 288-310. doi:

10.1080/02560046.2013.800665.

Carlson, R.G., Jones, K.D. (2010). Continuum of Conflict and Control: A conceptualization of intimate partner violence typologies. The Family Journal: Counseling and Therapy for Couples and Families, 18(3), 248254. doi: $10.1177 / 1066480710371795$.

${ }^{*}$ Carter-Visscher, R.M., Naugle, A.E., Bell, K.M., Suvak, M.K. (2007). Ethics of asking trauma-related questions and exposing participants to arousalinducing stimuli. J Trauma Dissociation, 8(3), 27-55.

Cattaneo, L.B., Bell, M.B., Goodman, L.A., Dutton, M.A. (2007). Intimate partner violence victims' accuracy in assessing their risk for re-abuse. Journal of Family Violence, 22, 429-440. doi: 10.1007/s10896-007-9097-8.

Centers for Disease Control \& Prevention (2014). Intersection of intimate partner violence and HIV in women. February, Retrieved from http://www.cdc.gov/violenceprevention/pdf/ipv/13_243567_green_aaga.pdf

Centers for Disease Control and Prevention. (2015a). CDC in South Africa. Center for Global Health. Retrieved from 
http://www.cdc.gov/globalhealth/countries/southafrica/pdf/southafrica_factsheet.pdf

Centers for Disease Control and Prevention. (2015b). Intimate partner violence. Retrieved from http://www.cdc.gov/violenceprevention/intimatepartnerviolence/ Chaudoir, S.R., Fisher, J.D., \& Simoni, J.M. (2011). Understanding HIV disclosure: A review and application of the disclosure processes model. Social Science \& Medicine, 72, 1618-1629. doi: 10.1016/j.socscimed.2011.03.028.

Chibber, K.S. \& Krishnan, S. (2011). Confronting intimate partner violence: a global health priority. Mount Sinai Journal of Medicine, 78, 449-457. doi: 10.1002/msj.20259.

Chimphamba Gombachika, Fjeld, H., Chirwa, E., Sundby, J., Malata, A., Maluwa, A. (2012). A social ecological approach to exploring barriers to accessing sexual reproductive health services among couples living with HIV in southern Malawi. International Scholarly Research Network, 2012, 1-14. Article ID 825459. doi: 10.5402/2012/825459.

Christofides, N. \& Jewkes, R. (2010). Acceptability of universal screening for intimate partner violence in voluntary HIV testing and counseling services in South Africa and service implications. AIDS Care. 22(3), 279-285. doi: $10.1080 / 09540120903193617$.

Christopoulos, K.A., Massey, A.D., Lopez, A.M., Geng, E.H., Johnson, M.O., Pilcher, C.D., Fielding, H., Dawson-Rose, C. (2013). "Taking a half day at 
a time:" Patient perspectives and the HIV engagement in care continuum. AIDS Patient Care and STDs, 27(4), 223-230. doi:

10.1089/apc.2012.0418.

${ }^{*}$ Coker, A.L., Derrick, C., Lumpkin, J.L., Aldrich, T.E. \& Oldendick, R. (2000). Help-seeking for intimate partner violence and forced sex in South Carolina. American Journal of Preventative Medicine, 19(4), 316-320.

Dass-Brailsford, P. (2007). A practical approach to trauma: Empowering interventions. Sage Publications. United States of America.

*Davies, K., Block, C.R. \& Campbell, J. (2007). Seeking help from the police: Battered women's decisions and experiences. Criminal Justice Studies: A Critical Journal of Crime, Law \& Society, 20(1), 15-41.

Davies, P.J. \& Dryer, Y. (2014). A pastoral psychological approach to domestic violence in South Africa. HTS Teologiese Studies/Theological Studies, 70(3), 1-8. http://dx.doi.org/10.4102/hts.v70i3.2802.

Davis, K. (2012). Understanding and addressing stigma and attachment insecurity in HIV-positive women who experience intimate partner violence: A model of medical and psychosocial care. Social Work in Health Care, 51, 213-231. doi: 10.1080/00981389.2011.614835.

Decker, M.R., Frattaroli, S., McCaw, B., Coker, A.L., Miller, E., Sharps, P., Lane, W.G., Mandal, M., Hirsch, K., Strobino, D.M., Bennett, W.L., Campbell, J., \& Gielen, A. (2012). Transforming the healthcare response to intimate partner violence and taking best practices to scale. Journal of Women's Health, 21(12), 1222-1229. doi: 10.1089/jwh.2012.4058. 
Department of Justice. (Jan, 2015). Intimate partner violence risk assessment tools: a review. Retrieved from http://www.justice.gc.ca/eng/rp-pr/cj-jp/fvvf/rr12_8/p3.html\#ftn5

Dienemann, J., Campbell, J., Landenberger, K., Curry, M.A., (2002). The domestic violence survivor assessment: A tool for counseling women in intimate partner violence relationships. Patient Education and Counseling, 46, 221-228. 0738-3991/02/\$.

Dlamini, P.S., Kohi, T.W., Uys, L.R., Phetlhu, R.D., Chirwa, M.L., Naidoo, J.R., Holzemer, W.L., Greef, M., Makoae, L.N. (2008). Verbal and physical abuse and neglect as manifestations of HIVIAIDS stigma in five African countries. Public Health Nursing, 24(5), 389-399.

Dunkle, K.L. \& Decker, M.R. (2012). Gender-based violence and HIV: reviewing the evidence for links and causal pathways in the general population and higher-risk groups. American Journal of Reproductive Immunology, 69(1), 20-26. doi: 10.1111/aji.12039.

Dunkle, K.L., Jewkes, R.K., Brown, H.C., Gray, G.E., McIntryre, J.A., Harlow, S.D. (2004). Gender-based violence, relationship power, and risk of HIV infection in women attending antenatal clinics in South Africa. The Lancet, $363,1415-1421$.

*El-Khoury, M.Y., Dutton, M.A., Goodman, L.A., Engel, L., Belameric, R.J., \& Krause, E. (2004). Ethnic differences in battered women's formal helpseeking strategies: A focus on health, mental health, and spirituality. Cultural Diversity \& Ethnic Minority Psychology, 10(4), 383-393. 
Elul, B., Lahuerta, M., Abacassamo, F., Lamb, M.R., Ahoua, L., McNairy, M.L., Tomo, M., Horowitz, D., Sutton, R., Mussa, A., Gurr, D., Jani, I. (2014). A combination strategy for enhancing linkage to and retention in HIV care among adults newly diagnosed with HIV in Mozambique: study protocol for a site randomized implementation science study. BMC Infectious Disease, 14, 549-569. doi: 10.1186/s12879-014-0549-5.

Ending Violence Association of BC. (2013). Safety planning across culture \& community: a guide for front line violence against women responders. Community Coordination for Women's Safety.

Faul, A. \& Lawson, T. (2012). Untitled. Chapter selection: Section II, Chapter 2. Independent Samples. Archives of Kent School of Social Work, University of Louisville. Louisville, Kentucky.

Garcia-Moreno, C., Hansen, H.A.FM., Ellsberg, M., Heise, L. \& Watts, C. (2005). WHO multi-country study on women's health and domestic violence against women. World Health Organization. Geneva, Switzerland. Retrieved from http://www.unece.org/fileadmin/DAM/stats/gender/vaw/surveys/Serbia $\% 20$ \%26\%20Montenegro/WHO\%20VAW\%20multiple\%20country.pdf Gielen, A.C., Ghandour, R.M., Burke, J.G., Mahoney, P., McDonnell, K.A., \& O'Campo, P. (2007). HIVIAIDS and intimate partner violence: intersecting women's health issues in the United States. Trauma, Violence \& Abuse, 8(2), 178-198. doi: 10.1177/1524838007301476. 
Gielen, A.C., McDonnell, K.A., Burke, J.G., O'Campo, P. (2000). Women's lives after an HIV diagnosis: disclosure and violence. Maternal and Child Health Journal, 4(2), 111-120. 1092-7875/00/0600-0111\$18.00/0

Glass, N., Eden, K.B., Bloom, T., \& Perrin, N. (2010). Computerized aids improves safety decision process for survivors of intimate partner violence. Journal of Interpersonal Violence, 25(11), 1947-1964. Doi: 10.1177/0886260509354508.

*Goodkind, J.R., Sullivan, C.M., \& Bybee, D.I. (2004). A contextual analysis of battered women's safety planning. Violence Against Women, 10(5), 514533.

Goodman, L., Dutton, M.A., Weinfurt, K., \& Cook, S. (2003). The intimate partner violence strategies index: development and application. Violence Against Women, 9(2), 163-186. doi: 10.1177/1077801202239004.

Govindassamy, D., Krazner, K., van Schaik, N., Noubary, F., Wood, R., Walensky, R. P., Freedberg, K.A., Bassett, I.V., Bekker, L. G. (2013). Linkage to HIV, TB and non communicable disease care from a mobile testing unit in Cape Town, South Africa. PLoS One, 8(11). E80017. doi: 10.1371/journal.pone.0080017.

Gumani, M. \& Mudhovozi, P. (2013). Gender-based violence: opportunities and coping resources for women in abuse unions. Gender \& Behavior, 11(2), 5569-5578. ISSN: 1596-9231.

Hale, F. \& Vazquez, M. (2011). Violence against women living with HIV/AIDS: a background paper. Development Connections. USA. 
Halket, M.M., Gormley, K., Mello, N., Rosenthal, L., \& Mirkin, M.P. (2014). Stay with or leave the abuser? The affects of domestic violence victim's decision on attribution made by young adults. Journal of Family Violence, 29, 35-49. doi: 10.1007/s10896-013-9555-4.

Hardesty, J.L., Crossman, K.A., Haselschwerdt, M.L., Raffaelli, M., Ogolsky, B.G., \& Johnson, M.P. (2015). Toward a standard approach to operationalizing coercive control \& classifying violence types. Journal of Marriage \& Family, 77(4), 833-843. doi: 10.1111/jomf/12201.

Harding, H.G. \& Helweg-Larsen M. (2008). Perceived risk for future intimate partner violence among women in a domestic violence shelter. Journal of Family Viol, 24, 75-85. doi: 10.1007/s10896-008-9211-6.

Heckert, D.A. \& Gondolf, E.W. (2004). Battered women's perceptions of risk versus risk factors and instruments in predicting repeat reassault. Journal of Interpersonal Violence, 19, 778-800. doi: 10.1177/0886260504265619. Heise, L.L. (1998). Violence against women: An integrated, ecological framework. Violence Against Women, 4(3), 262-290. doi: $10.1177 / 1077801298004003002$.

Home of the Duluth Model (2011). What is the Duluth Model. Retrieved from http://www.theduluthmodel.org/about/

Hung, K.J, Scott, J., Ricciotti, H.A., Johnson, T.R., Tsai, A.C. (2012). Communitylevel and Individual-level influences of intimate partner violence on birth spacing in sub-Saharan Africa. Obstetrics \& Gynecology, 119(5), 975-982. ISSN: 0029-7844/12. 
Jenness, S.M, Myers, J.E., Neaigus, A., Lulek, J., Navejas, M., Raj-Singh, S. (2012). Delayed entry into HIV medical care after HIV diagnosis: Risk factors and research methods. AIDS Care, 24(10), 1240-1248. http://dx.doi.org/10.1080/09540121.2012.656569. ISSN: 0954-0121. Jewkes, R. (2013). Intimate partner violence: The end of routine screening. The Lancet. www.thelancet.com. doi: 10.1016/S0140-6736/(13)60584-X. Jewkes, R., Dunkle, K., Nduna, M., Levin, J., Khuzwayo, N., Koss, M., Puren, A., Duvvury, N. (2006). Factors associated with HIV sero-status in young rural South African Women: Connections between intimate partner violence and HIV. International Journal of Epidemiology. 35(6), 1461-1468.

Jewkes, R., Levin, J. \& Penn-Kekana, L. (2002). Risk factor for domestic violence: Findings from a South African cross-sectional study. Social Science \& Medicine, 55, 1603-1617.

Jewkes, R., Sikweyiya, Y., Morell, R., \& Dunkle, K. (2011). The relationship between intimate partner violence, rape and HIV amongst South African men: A cross-sectional study, PLoS One, 6(9), e24256. doi: 10.1371./journal.pone.0024256.

Jewkes, R., Watts, C., Abrahams, N., Penn-Kekana, L., \& Garcia-Moreno, C. (2000). Ethical and methodological issues in conducting research on gender-based violence in southern Africa. Research Methodology, Reproductive Matters, 8(15), 1-12.

Johnson, M.P., Leone, J.L. (2005). The differential effects of intimate terrorism and situational couple violence: Findings from the National Violence 
Against Women Survey. Journal of Family Issues, 26(3), 322-349. doi:

10.1177/0192513X04270345.

Jones, D.J., Beach, S.R.H., Forehand, R., \& The Family Healthy Project

Research Group. (2003). Partner abuse and HIV infection: Implications for psychosocial adjustment in African American women. Journal of Family Violence, 18(5), 257-268. 0885-7482/03/1000-0257/0.

Jones, D.L., Weiss, S.L., Malow, R., Ishii, M., Devieux, J., Stanley, H., Cassells, A., Tobin, J.N., Brondolo, E., LaPerriere, A., Efantis-Potter, J., O'Sullivan, M.J., Schneiderman, N. (2001). A brief sexual intervention for women livings with AIDS: Acceptability, use, and ethnicity. Journal of Urban Health: Bulletin of the New York Academy of Medicine, 78(4), 593-604.

Joyner, K. \& Mash, B. (2012). A comprehensive model for intimate partner violence in South African primary care: Action research. Bio Med Central Health Services Research, 12, 399. http://www.biomedcentral.com/1472$6963 / 12 / 399$.

Kayigamba F.R., Bakker, M.I., Fikse, H., Mugisha, V., \& Asiimwe, A. 2012. Patient enrolment into HIV care and treatment within 90 days of HIV diagnosis in eight Rwandan health facilities: A review of facility-based registers. PLoS One, 7(5): 1-9. doi: 10.1371/journal.pone.0036792.

Kahana, M.J. \& Miller, J.F. (2013). Memory, recall dynamics. Retrieved from http://memory.psych.upenn.edu/files/pubs/KahaMill13.pdf

Kendall, J., Pelucio, M.T., Casaletto, J., Thompson, K.P., Barnes, S., Petit, B., Aldrich, M. (2009). Impact of emergency department violence intervention. 
Journal of Interpersonal Violence, 24(2), 280-306. doi:

$10.1177 / 0886260508316480$.

Kennedy, C.E., Haberlen, S., Amin, A., Baggaley, R., \& Narasimhan, (2015).

Safer disclosure of HIV serostatus for women living with HIV who experience or fear violence: A systematic review. Journal of the International AIDS Society, 18(5), 20292.

http://dx.doi/10.7448/IAS.18.6.20292.

Kenyon, C., Buyze, J. \& Colebunders, R. (2013). HIV prevalence by race covaries closely with concurrency and number of sex partners in South Africa. PLoS One, 8(5), e64080. doi: 10.371/journal.pone.0064080.

Khumalo, Sakutukwa, G., Morin, S.F., Fritz, K., Charlebois, E.D., van Rooyen, H., Chingono, A., Modiba, P., Mrumbi, K., Visrutaratna, S., Coates, B. (2008). Project accept (HPTN 043): A community-based intervention to reduce HIV incidence in populations at risk for HIV in sub-Saharan Africa and Thailand. J Acquir Immune Defic Syndr, 49(4), 422-431. doi: 10.1097/QAI.0b013e31818a6cb5.

Kilembe, W., Wall, K.M., Mokgoro, M., Mwaanga, A., Dissen, E., Kamusoko, M., Phiri, H., Sakulanda, J., Davitte, J., Reddy, T., Brockman, M., Ndung'u, T., Allen, S. (2015). Knowledge of HIV serodiscordance, transmission, and prevention among couples in Durban, South Africa. PLoS One, 10(4), e0124548. doi: 10.1371/journal.pone.0124548.

Kouyoumdjian, F.G., Calzavara, L.M., Bondy, S.J., O'Campo, P, Serwadda, D., Nalugoda, F., Kagaayi, J., Kigozi, G., Wawer, M., Gray, R. (2013). 
Intimate partner violence is associated with incident HIV infection in women in Uganda. AIDS, 27(8). 1331-1338. International Perspectives on Sexual \& Reproductive Health, 2013, Digest, 39(3), 165-166.

Krazner, K., Govindassamy, D., van Schaik, N., Thebus, E., Davies, N., Zimmermann, M. Jeneker, S., Lawn, S., Wood, R., Bekker, L.G. (2012). Incentivized recruitment of a population sample to a mobile HIV testing service increases the yield of newly diagnosed cases, including those in need of antiretroviral therapy. HIV Medicine, 13, 132-137. doi:

10.1111/j.1468-1293.2011.00947.x

Krazner, K., Zeinecker, J., Ginsbert, P., Orrell, C., Kalawe, N., Lawn, S., Bekker, L-G., Wood, R. (2010). Linkage to HIV care and antiretroviral therapy in Cape Town, South Africa. PLoS One, 5(11), e13801. doi: 10.1371/journal.pone.0013801.

Krohne, H.W. (2002). Stress and coping theories. Johannes GutenbergUniversität Mainz Germany.

Landenberger, K. (1998). The dynamics of leaving and recovering from an abusive relationship. Clinical Issues, 27(6), 700-707.

Larson, B.A., Brennan, A., McNamara, L., Long, L. Rosen, S., Sanne, I., Fox, M.P. (2010). Lost opportunities to complete CD4+ lymphocyte testing among patients who test HIV positive for HIV in South Africa. Bull World Health Organization, 88, 675-680. doi: 10.2471/BLT.09.068981.

Lawn, S.D., Myer, L., Harling, G., Orrell, C., Bekker, L-G., Wood, R. (2006). Determinants of mortality and nondeath losses from antiretroviral 
treatment service in South Africa: Implications for program evaluation. HIVIAIDS, 43, 770-776. 1058-4838/2006/4306-0018\$15.00

Lawson, J. (2012). Sociological theories of intimate partner violence. Journal of Human Behavior in the Social Environment, 22, 572-590. doi: 10.1080/10911359.2011.598748.

Le Roux-Kemp, A. (2013). HIVIAIDS, to disclose or not to disclose: That is the question. Potchefstroom Electronic Law Journal. 16(1), 201-239.

Lewis, C.C., Fischer, S., Weiner, B.J., Stanick, C., Kim, M. \& Martinez, R.G. (2015). Outcomes for Implementation Science: An enhanced systematic review of instruments. Implementation Science, 10(155). doi: 10.1186/s13012-015-0342-x.

Lindhorst, T., Nurius, P., Macy, R.J. (2005). Contextualized assessment with battered women: strategic safety plan to cope with multiple harms. Journal of Social Work Education, 41(2), 331-352.

Lippman, S.A., Maman, S., MacPhail, C., Twine, R., Peacock, D., Kahn, K., Pettifor, A. (2013). Conceptualizing community mobilization for HIV prevention: Implications for HIV prevention programming in the African context. PLOS One, 8(10), 1-13. doi: 10.1371/journal.pone.0078208 Losina, E., Bassett, I., Giddy, J., Chetty, S., Regan, S., Walensky, R. P., Ross, D., Scott, C.A., Uhler, L.M., Katz, J.N., Holst, H., \& Freedberg, K. A. (2010). The "ART" of linkage: Pre-treatment loss to care after HIV diagnosis at two PEPFAR sites in Durban, South Africa. PLoS One, 5(3), e9538. doi: 10.1371/journal.pone.0009538. 
Mabuto, T., Latka, M.H., Kuwane, B., Churchyard, G.J., Charalambous, S., Hoffman, C.J. (2014). Four models of HIV counseling and testing: Utilization and test results in South Africa, PLoS One, 9(7), e102267. doi: 10.1371/journal.pone.0102267.

MacPherson, P., Corbett, E.L., Makombe, S.D., van Oosterhout, J.J., Manda, E., Choko, A.T., Thindwa, D., Swuire, B., Mann, G.H., Lalloo, D.G. (2012). Determinates and consequences of linkage to antiretroviral therapy at primary care level in Blantyre, Malawi: A prospective cohort study. PLoS One, 7(9), 1-10. doi: 10.1371/journal.pone.0044794.

Mall, S., Middlekoop, K., Mark, D., Wood, R., Bekker, L-G. (2013). Changing patterns in HIVIAIDS stigma and uptake of voluntary counselling and testing services: The result of two consecutive community surveys conducted in the Western Cape, South Africa. AIDS Care, 25(2), 194-201. http://dx.doi.org/10.1080/09540121.2012.689810.

Maman, S., Mbwambo, J., Hogan, N.M., Kilonzo, G.P., \& Sweat, M. (2001). Women's barriers to HIV-1 testing and disclosure: Challenges for HIV-1 voluntary counselling and testing. AIDS Care, 13(5), 595-603. doi: $10.1080 / 09540120120063223$.

*Maman, S., Moodley, D., McNaughton-Reyes, H.L., Groves, A.K., Kagee, A., Moodley, P. (2014). Efficacy of enhanced HIV counseling for risk reduction during pregnancy and in the postpartum period: A randomized controlled trial. PLoS One, 9(5), e97092.

Maman, S., Mwambo, J., Hogan, N.M., Kilonzo, G.P., \& Sweat, M. (2002). HIV- 
positive women report more lifetime violence: findings from a voluntary counseling and testing clinic in Dar es Saleem, Tanzania. American Journal of Public Health. Research and Practice, 92(8), 1331-1337.

Maman, S., Yamanis, T., Kouyoumdjian, F., Watt, M., Mbwambo, J. (2010). Intimate partner violence and the association with HIV risk behaviors among young men in Dar es Salaam, Tanzania. Journal of Interpersonal Violence, 25(10), 1855-1872.

Matseke, G \& Peltzer, K. \& Habil (2013). Screening and brief itervention for intimate partner violence among antenatal care attendees at primary healthcare clinics in Mpumalanga Province, South Africa. South African Journal of Obstetrics \& Gynecology, 19(2), 40-43. doi: 10.7196/SAJOG.637.

May, C. (2013). Towards a general theory of implementation. Implementation Science, 8(18). 1-15.

Mayer, K.H. (2011). Introduction: linkage, engagement, and retention in HIV care: Essential for optimal individual- and community-level outcomes in the era of highly active antiretroviral therapy. Clin Infect Dis, 52, 205-207. doi: 10.1093/cid/ciq043.

Medley, A., Garcia-Moreno, C., McGill, S., \& Maman, S. (2004). Rates, barriers \& outcomes of HIV serostatus disclosure among women in developing countries: Implications for prevention of mother-to-child transmission programmes. Bulletin of the World Health Organization, 82(4), 299-310. Ref. No. 03-003913. 
Messing, J.T. \& Thaller, J. (2013). The Average predictive validity of intimate partner violence risk assessment instruments. Journal of Interpersonal Violence, 28(7), 1537-1558. doi: 10.1177/0886260512468250.

Miller, W.R, \& Rollnick, S. (2002). Motivational Interviewing: Preparing people for change. Second Ed. Guilford, New York NY.

Mkandawire-Valhmu, L., Stevens, P.E., Kako, P.M., Dressel, A. (2013). Safety planning in focus groups of Malawian women living with HIV: Helping each other deal with violence and abuse. Violence Against Women, 19(11): 1331-1349. doi: 10.1177/1077801213514859.

Mogale, R.S., Burns, K.K., \& Richter, S. (2012). Violence against women in South Africa: Policy position and recommendation. Violence Against Women, 18(5), 580-594. doi: 10.1177/1077801212453430.

Mugglin, C., Estill, J., Wandeler, G., Bender, N., Egger, M., Gsponer, T., Keiser, O. (2012). Loss to programme between HIV diagnosis and initiation of antiretroviral therapy in sub-Saharan Africa: Systematic review and metaanalysis. Tropical Medicine and International Health, 17(12), 1509-1520. doi: 10.1111/j.1365-3156.2012.03089.x

Myer, L., Rebe, K., Morroni, C. (2007). Missed opportunities to address reproductive health care needs among HIV-infected women in antiretroviral therapy programmes. Tropical Medicine and International Health, 12(12), 1484-1489. doi: 10.1111/j.1365-3156.2007.01955.x.

NAM AIDSmap. (2016). The Health-belief model. Retrieved from http://www.aidsmap.com/The-health-belief-model/page/1768382/ 
National Institute of Health. (2008). FAQ ClinicalTrials.gov. Clinical Trial Phase. US National Library of Medicine. April, Retrieved from https://www.nlm.nih.gov/services/ctphases.html

National Network to End Domestic Violence. (2014). Positively safe: the intersection of domestic violence and HIVIAIDS. HIVIAIDS Power and Control Wheel.

Nilsen, P. (2015). Making sense of implementation theories, models and frameworks. Implementation Science, 10(53), 1-13. doi: 10.1186/s13012015-0242-0.

*O'Campo, P., McDonnell, K., Gielen, A., Burke, J., \& Chen, Y. (2002). Surviving physical and sexual abuse: what helps low-income women? Patient Education \& Counseling, 46(3), 205-212.

Ofstehage, A., Gandhi, A., Sholk, J., Radday, A., Stanzler, C. (2011). Empowering victims of domestic violence. Social Issue Report. Health and Well-Being, Boston, MA. WEB: http://rootcause.org/social-impactresearch.

Ozer, E.M. \& Bandura, A. (1990). Mechanisms governing empowerment effects: A self-efficacy analysis. Journal of Personality and Social Psychology, 58(3), 472-486.

Painter, T. M. 2001. Voluntary counseling and testing for couples: A highleverage intervention for HIVIAIDS prevention in sub-Saharan Africa. Social Science \& Medicine, 53, 1397-1411. 
Parker, E.M. \& Gielen, A.C. (2014). Intimate partner violence and safety strategy use: Frequency of use and perceived effectiveness. Women's Health Issues, 24-6, 584-593. doi: 10.1016/j.whi.2014.08.001.

Patten, G.EM., Wilkinson, L., Conradie, K., Isaakidis, P., Harries, A.D., Edgington, M.E., De Azevedo, V., van Cutsem, G. (2013). Impact on ART initiation of point-of-care CD4 testing at HIV diagnosis among HIV-positive youth in Khayelitsha, South Africa. Journal of International AIDS Society, 16. 18518. http://dx.doi.org/10.7448/IAS.16.1.18518.

Payne, D. \& Warmeling, L. (2009). Domestic violence and the female victim: The real reason women stay! Journal of Multicultural, Gender and Minority Studies, 3(1), 1-6.

Peltzer, K. (2013). Intimate Partner Violence Among New HIV Positive Mothers in South Africa. Gender and Behavior, 11(1).

Peltzer, K., \& Mlambo, G. (2013). HIV disclosure among HIV positive new mothers in South Africa. Journal of Psychology in South Africa, 23(2), 327334. ISSN 1433-0237.

Peltzer, K., Mashego, T-A., Mabeba, M. (2003). Attitudes and practices of doctors towards domestic violence victims in South Africa. Health Care for Women International, 24, 149-157. doi: 10.1080/07399330390178431.

Peltzer, K., Pengpid, S., McFarlane, J. \& Banyini, M. (2013). Evaluation of the effectiveness of protection orders for female victims of intimate partner violence in vhembe district of South Africa. Journal of Psychology in Africa, 23(3), 489-494. ISSN 1433-0237. 
Peterson, E. (2009). Addressing domestic violence: Challenges experienced by Anglican clergy in the diocese of Cape Town, South Africa, Social Work \& Christianity, 36(4), 449-469.

Pronyk, P.M., Hargreaves, J.R., Kim, J. C., Morison, L.A., Phetla, G., Watts, C., Busza, J., Porter, J.D.H. (2006). Effect of a structural intervention for the prevention of intimate-partner violence and HIV in rural South Africa: A cluster randomized trial. The Lancet, 368, 1973-83. doi: 10.1016/S01406736(06)69744-4.

Prowse, K.M., Logue, C.E., Fantasia, H.C., Sutherland, M.A. (2013). Intimate partner violence and the CDC's best-evidence HIV risk reduction interventions. Public Health Nursing, 31(3), 215-233. doi: 10.1111/phn.12076.

Ramachandran, S., Yonas, M.A., Silvestre, A.J., Burke, J.G. (2010). Intimate partner violence among HIV positive persons in an urban clinic. AIDS Care, 22(12), 1536-1543. doi: 10.1080/09540121.2010.482199.

Reed, J.B., Hanson D, McNaghten, A.D., Bertolli, J., Teshale, E., Gardner, L., \& Sullivan, P. (2009). HIV testing factors associated with delayed entry into HIV medical care among HIV-infected persons from eighteen states, United States, 2000-2004. AIDS Patient Care and STDs, 23(9), 765-776. doi: 10.1089/apc.2008.0213.

Rees, K., Zweigenthal, V., \& Joyner, K. (2014). Health sector responses to intimate partner violence: A literature review. Afr J Prm Health Care Fam Med, 6(1), 1-9. http://dx.doi.org/10.4102/phcfm.v6i1.712. 
Roehl, J., O'Sullivan, C., Webster, D., Campbell, J. (2005). Intimate partner violence risk assessment validation study, Final Report. Research report submitted to the U.S. Department of Justice. NIJ 2000WTVX0011.

Rogers, E.M. (1997). Diffusion of Innovations Theory. Retrieved from http://www.indiana.edu/ t581qual/Assignments/Diffusion_of_Innovations.p df

Rosen, S. \& Fox, M.P (2011). Retention in HIV care between testing and treatment in sub-Saharan Africa: A systematic review. PLoS One, 8(7) 116. doi: 10.1371/journal.pmed.1001056.

Rubin, A. \& Babbie, E.R. (2008). Research methods for Social Work. Sixth Edition. Thomson Brooks/Cole.

Saunders, D.G., Holter, M.C., Pahl, L.C., Tolman, R.M., Kenna, C.E. TANF workers' responses to battered women and the impact of brief worker training. (2005). Violence Against Women, 11(2), 227-254. doi: $10.1177 / 1077801204271837$.

Schochetman, G. \& George, G.J. (1994). AIDS testing: A comprehensive guide to technical, medical social, legal and management issues. Ed. 2. Springer-Verlag, New York.

Shafer, K.R., Brant, J., Gupta, S., Thorpe, J., Winstead-Derlega, C., Pinkerton, R., Laughon, K., Ingersoll, K., Dillingham, R. (2012). Intimate partner violence: a predictor of worse HIV outcomes and engagement in care. AIDS Patient Care and STDs, 26, 356-365. doi: 10.1089/apc.2011.0409. 
Shai, N.J., Jewkes, R., Nduna, M., Dunkle, K. (2012). Masculinities and condom use patterns among young rural South African men: A cross-sectional baseline survey. BMC Public Health. BioMed Central, 12, 462. Doi: 10.1186/1471-2458-12-462. http://www.biomedcentral.com/1471$2458 / 12 / 462$.

*Shannon, L., Logan, T., Cole, J., \& Medley, K. (2006). Help-seeking and coping strategies for intimate partner violence in rural and urban women. Violence and Victims, 21(2), 167-181.

Shi, C-F., Kouyoumdjian, F.G., Dushoff, J. (2013). Intimate partner violence is associated with HIV infection in women in Kenya: A cross-sectional analysis. BMC Public Health, 13, 512. Doi: 1471-2458/13/512.

Snyder, K., Wallace, M., Duby, Z., Aquino, L.D.H., Stafford, S., Hosek, S. Futterman, D., Bekker, L-G. (2014). Preliminary results from hlangnani (coming together): A structured support group for HIV infected adolscents piloted in Cape Town, South Africa. Children and Youth Services Review, 45, 114-121. Doi: 10.1016/j.childyouth.2014.03.027.

Statistics South Africa (2011). Statistical release (Revised): Census 2011. P0301.4. Retrieved from http://www.statssa.gov.za/publications/P03014/P030142011.pdf

Straus, M.A., Hamby, S.L., Boney-McCoy, S., Sugarman, D.B. (1996). The revised Conflict Tactics Scales (CTS2) development and preliminary psychometric data. Journal of Family Issues, 17(3), 283-316. 
Swart, L-A., Seedat, M., Stevens, G.,. Ricardo, I. (2002). Violence in adolescents' romantic relationships: Findings from a survey amongst school-going youth in a South African Community. Journal of Adolescence, 25, 385395. 10.1006/jado.2002.0483.

Tam, M., Amzel, A. \& Phelps, B.R. (2015). Disclosure of HIV serostatus among pregnant and postpartum women in sub-Saharan Africa: A systematic review. AIDS Care, 27(4), 436-450. Doi: 10.1080/09540121.2014.997662.

Thabane, L., Ma, J., Chu, R., Cheng, J., Ismaila, A., Rios, L.P., Robson, R., Thabane, M., Giangregorio, L., Goldsmith, C.H. (2010). A Tutorial on Pilot Studies: the what, why and how. BioMed Central. Medical Research Methodology, 10(1), 1-10. Doi: 1471-2288/10/1.

Thackeray, J., Stelzner, S., Downs, S.M., Miller, C. (2007). Screening for intimate partner violence: The impact of screener and screening environment on victim comfort. Journal of Interpersonal Violence, 22(6), 659-670. Doi: $10.1177 / 0886260507300206$.

Treatment Action Group. (2013). Revitalizing the U.S. national HIVIAIDS strategy. Meeting Report. December 11-12, 2012. Washington, D.C. New York, NY. April 2013. Retrieved from http://www.treatmentactiongroup.org/sites/tagone.drupalgardens.com/files/ 201304/RevitalizNHASMeetingReport.pdf

UNAIDS. (2000). Voluntary Counselling and Testing (VCT). UNAIDS technical update. Joint United Nations Program on HIVIAIDS. UNAIDS Information Center, Geneva, Switzerland. WC 503.6. Retrieved from 
http://www.unaids.org/sites/default/files/en/media/unaids/contentassets/da taimport/publications/irc-pub01/jc379-vct_en.pdf

UNAIDS. (2012). Global report: UNAIDS report on the global AIDS epidemic 2012. Joint United Nations Programme on HIVIAIDS (UNAIDS). ISBN 978-92-9173-592-1. Retrieved from http://www.unaids.org/sites/default/files/media_asset/20121120_UNAID S_Global_Report_2012_with_annexes_en_1.pdf

UNAIDS. (2013a). Efficient \& sustainable HIV responses: Case studies on country progress. Joint United Nations Programme on HIVIAIDS (UNAIDS). UNAIDS/JC2450. ISBN 978-92-9253-007-5. Retrieved from http://www.unaids.org/sites/default/files/media_asset/JC2450_casestudies-country-progress_en_0.pdf

UNAIDS. (2013b). Global report: UNAIDS report on the global AIDS epidemic 2013. Joint United Nations Programme on HIVIAIDS (UNAIDS). ISBN 978-92-9253-032-7. Retrieved from http://www.unaids.org/sites/default/files/media_asset/UNAIDS_Global_Re port_2013_en_1.pdf

UNAIDS. (2015a). Global AIDS response progress reporting 2015. World Health Organization and UNAIDS 2015. ISBN 978-92-9253-072-3. Retrieved from http://www.unaids.org/sites/default/files/media_asset/JC2702_GARPR201 5guidelines_en.pdf 
UNAIDS. (2015b). Staying ahead of the AIDS epidemic in South Africa.

Retrieved from

http://www.unaids.org/en/resources/presscentre/featurestories/2015/octob er/20151008_SA_president

Van der Kop, M.L., Ojakaa, D.L., Patel, A., Thabane, L., Kinagawi, K., Ekström, A.M., Smillie, K., Karanja, S., Awiti, P., Mills, E., Marra, C., Kyomuhangi, L.B., Lester, R.T. (2013). The effect of weekly short message service communication on patient retention in care in the first year after HIV diagnosis: Study protocol for a randomised controlled trial (WelTel Retain). BMJ Open, 3, e003155. doi: 10.1136//bmjopen-2013-003155.

Van Schalkwyk, S., Boonzaier, F., \& Gobodo-Madikizela, P. (2014). 'Selves' in contradiction: Power and powerlesness in South African shelter residents' narratives of leaving abusive heterosexual relationships. Feminism \& Psychology, 24(3), 314-331. Doi: 10.1177/0959353513514245.

Van Zyl, M.A., Barney, R.J. (2014). Online adolescent HIVIAIDS prevention: The impact of race on program outcomes. Journal of Ethnic and Cultural Diversity in Social Work, 23, 78-92. Doi: 10.1080/1531204.2013.871994

Van Zyl, M.A., Brown, L.L., Pahl, K. (2013). Youth attitudes, perceptions and behaviors around gender norms and gender based violence in South Africa. Technical Report submitted to the Swedish Government.

Van Zyl, M.A., Brown, L.L., Pahl, K. (2015). Using a call center to encourage linkage to care following mobile HIV counseling and testing. AIDS Care, 27(7), 921-925. Doi: 10.1080/09540121.2015.1015483. 
Van Zyl, M.A., Barney, R.J. \& Pahl, K. (2014). Voluntary counselling and testing and celebrity-based HIVIAIDS prevention education: A pilot program implemented in Cape Town secondary schools. Journal of HIVIAIDS \& Social Services, 13, 292-305. Doi: 10.1080/15381501.2013.864174.

Vaughn, P.W., Rogers, E.M., Singhal, A. \& Swalehe, R.M. (2000). Entertainmenteducation and HIVIAIDS prevention: A field experiment in Tanzania. $J$ Health Communication, 5(81), 81-100.

Vetten, L. (2005). Addressing domestic violence in South Africa: Reflections on strategy and practice. Violence Against Women: Good practices in combatting and eliminating violence against women. Expert Group Meeting. UN Division for the Advancement of Women, Vienna, Austria. Retrieved from http://www.un.org/womenwatch/daw/egm/vaw-gp2005/docs/experts/vetten.vaw.pdf

*Wagman, J.A., Gray, R.H., Campbell, J.C., Thoma, M. Ndyanabo, A., Ssekasanvu, J., et al. (2015). Effectiveness of an integrated intimate partner violence and HIV prevention intervention in Rakai, Uganda: Analysis of an intervention in an existing cluster randomized cohort. Lancet Global Health, 3(1). e23-33.

Wagman, J.A., Namatovu, F., Nalugoda, F., Kiwanuka, D., Nakigozi, G., Gray, R., Mawer, M.J., Serwadda, D. (2013). A public health approach to intimate partner violence prevention in Uganda: The SHARE project, Violence Against Women, 18(12), 1390-1412. Doi: $10.1177 / 1077801212474874$. 
Walsh, J. (2013). Theories for direct Social Work practice. $3^{\text {rd }}$ Edition. Cengage Learning, United States of America.

*Wiist, W.H., \& McFarlane, J. (1998). Utilization of police by abused pregnant Hispanic women. Violence Against Women, 4(6), 677-693.

Wong, F.Y., Huang, J., DiGangi, J.A., Thompson, E.E., Smith, B.D. (2008). Abuse, sexual risks, and depression among a sample of South Africans in Cape Town, South Africa. AIDS Education and Prevention, 20(1), 56-64.

Wong, L.H., van Rooyen, H., Modiba, P., Richter, L., Gray, G., Mclntyre, J.A., Schetter, C.D., \& Coates, T. (2009). Test and Tell: Correlates and consequences of testing and disclosure of HIV status in South Africa (HPTN 043 Project Accept). J Acquir Immune Defic Syndr, 50(2), 215-222.

World Health Organization (n.d.). Violence against women and HIVIAIDS: Critical intersections: Intimate partner violence and HIVIAIDS. The Global Coalition on Women and AIDS. UNAIDS initiative. Information Bulletin Series. Retrieved from http://data.unaids.org/GCWA/gcwa_fs_vaw3_who_01may05_en.pdf

World Health Organization (2001). Putting women first: Ethical and safety recommendations for research on domestic violence against women. WHO/FCH/GWH/01.1. Retrieved from http://www.who.int/gender/violence/womenfirtseng.pdf World Health Organization (2012). Understanding and addressing violence against women: Intimate partner violence. WHO/RHR/12.36. Retrieved from 
http://apps.who.int/iris/bitstream/10665/77432/1/WHO_RHR_12.36_eng.p df

World Health Organization. (2013). Responding to intimate partner violence and sexual violence against women: WHO clinical and policy and guidelines. Geneva, Switzerland. ISBN: 978924154859 5. Retrieved from http://www.who.int/reproductivehealth/publications/violence/97892415485 95/en/

Wright, R., Kiguwa, P., \& Potter, C. (2007). The Significance of sheltering in the lives of four women affected by abusive relationships. South African Journal of Psychology, 37(3), 616-637. ISSN 0081-2464.

Wynberg, E., Cooke, G., Shroufi, A., Reid, S.D., Ford, N. (2014). Impact of pointof-care CD4 cell testing on linkage to HIV care: A systematic review. Journal of the International AIDS Society, 17, 18809. Doi: 10.7448/IAS.17.1.18809.

Zacarias, A.E., Macassa, G., Svanstrom, L., Sores, J.JF., Antai, D. (2012). Intimate partner violence against women in Maputo City, Mozambique. BMC International Health and Human Rights, 12(35), 1-16.

Zembe, Y.Z., Townsend, L., Thorson, A., Silberschmidt, M., Ekstrom, A.M. (2015). Intimate partner violence, relationship power inequity, and the role of sexual and social risk factors in the production of violence among young women who have multiple sexual partners in a peri-urban setting in South Africa. PLoS One, 10(11). e0139430. doi: 10.1371/journal.pone.0139430. Zhang, A.H. \& Chen, L. (2013). Telephone Communication of HIV Testing 
Results for Improving Knowledge of HIV Infection Status. Public Health Nursing, 31(1), 58-59. doi: 10.1111/phn.12092. 
Appendix A

\section{Staff Explanation of Study Sheet}

\section{Reason for HIV IPV (intimate partner violence) Risk and Safety Study:}

$>$ You are very busy every day doing the extremely important work of HIV counseling and testing! The work is emotionally intense and exhausting. And, as it often goes with important work, there is more work to be done.

$>$ Shout-it-Now continues doing highly important work with testing individuals for HIV. And the links-to-care program, via our call centre, has helped S-N track if individuals are linking to care after testing HIV+ and encouraging them to do so.

$>$ This study builds on the work you are already doing and takes the work of the call centre one step further. We know young women have the worst rates for linking to HIV care after their diagnosis. We also know that one major barrier for women to receive HIV treatment is domestic violence. We want to find out which of our clients, who have just tested HIV+, are experiencing domestic violence and offer them extra support. This extra support will help them safely link to HIV treatment after their diagnosis.

$>$ On average, about $20-55 \%$ of women in South Africa are experiencing some type of domestic violence. In fact, South Africa has the highest rate of women killed by their partners in the world. Many of the women you have served in the past or even this week or today could be in grave danger, or at the very least, never receive the HIV treatment they need.

$>$ This study has been created so that women with HIV can receive the treatment they need. I am asking for your help in reaching the vulnerable young women who test HIV+ with Shout-it-Now.

$>$ Once a woman has tested HIV+ and you have had some time to do counseling with her during this very difficult time, please introduce this important study and ask for her participation.

$>$ The hope is that we can eventually offer this extra support all the time so that all individuals may successfully link to the HIV treatment they need after testing HIV+.

Thank you for your help! 
Appendix B

\section{Script by Staff for Study Recruitment}

(name of client)........, you heard some difficult news today and I want to remind you that Shout-it-Now will do everything possible to help you to get the right care and treatment. Remember, as I said, one of my colleagues will be calling you in the next 2 days to help you with this.

One of the things we worry about in South Africa is the high level of partner violence towards young women like you. We are working with a University in America to understand this problem and develop programs that will help to avoid these problems. Would you like to take part in this study? All that it will take is that one of the researchers will call you on your phone and ask you a few questions about what you think or have experienced about this. This will take about 15-20 minutes. Then, they will call you again in about two-four weeks time to ask you some follow-up questions. This will also take about 15 minutes. You don't have to answer anything that makes you feel uncomfortable and it will be completely confidential.

If you participate in this study, throughout the entire duration of the study, your name will be entered into a drawing for a free i-pod touch.

Are you OK about taking part in this study? Great, all I need now is for you to sign this form which lets us know that you have agreed to take part. Thanks so much! Your input will really help us to tackle the problem of partner violence!

Remember that Shout-it-Now will be in touch with you about your HIV results and will help you to get the care and treatment you need. 
Appendix C

\author{
Informed Consent English
}

\title{
Subject Informed Consent Document
}

\section{HIV Screening and Intimate Partner Violence Safety Study}

Sponsor(s) name \& address: University of Louisville, Louisville, KY and Shout-itNow, Cape Town, SA

Investigator(s) name \& address: Primary Investigator Michiel A. van Zyl and Coinvestigator Leslie Lauren Brown: 2217 South Third St. Louisville, KY 40292, USA.

Site(s) where study is to be conducted: Gauteng province and telephone calls will be made from Cape Town, South Africa.

Phone number for subjects to call for questions: 0217134414

\section{Introduction and Background Information}

You are invited to participate in a research study. The study is being conducted by Dr. Riaan van Zyl, PhD; Lauren Brown, LCSW, PhD Candidate and Shout-itNow. The study is sponsored by the University of Louisville, Department of Kent School of Social Work. The study will take place at all mobile HIV Counseling and Testing locations in Gauteng province that S-N visits within the timeframe of this study. Contact for this study will occur by phone, through the Shout-itNow call centre, which is located in Tokai, Western Cape. Approximately 225 subjects will be invited to participate.

\section{Purpose}

The purpose of this study is to see if women benefit from knowing their relationship safety score when screened for HIV and having a safety plan after been tested.

\section{Procedures}

In this study, you will be contacted by phone the day after receiving services with Shout-it-Now. Participation may occur in one of two phases.

If you are a participant in Phase I of this study, the phone interview or focus group you receive will only be used to help improve the study survey or intervention (content for phone discussion). We will contact you to get your opinion about survey questions and the phone discussion content. We want to know if you think the questions will be helpful, are beneficial, make you uncomfortable or if any changes are needed so people can better understand the 
questions or discussion and feel most satisfied with this process. Some or all of the questions from Phase II may be asked of you during this phase. These interviews or groups will not be audio recorded but, instead, your feedback will be written down by hand or typed into a computer.

If you are a participant in Phase II of this study, you will be asked questions in order to help determine how safe your relationship is from violence. Some questions were developed just for this study, and other questions are similar to questions routinely asked in different parts of the world to determine safety in relationships. Some questions relate to physical and sexual intimate partner violence and others emotional safety. There are also a few questions about experiences you may have had in the past with violent partners and your attitudes towards violence in a relationship. You will be contacted 2-3 times for this study and asked these questions during the first contact and the last contact. The first questions, in total, should take about 8-20 minutes to answer. There are 46 short questions in the first contact and 18-30 short questions in the final contact. Should any of the questions make you feel uncomfortable, you may choose to not answer them. You will continue to receive S-N services even if you do not answer all or any of the questions.

Participants in Phase II of this study will be randomized into different study groups so that not all participants will receive the same services.

\section{Potential Risks}

This study does have some possible risks, but most risks occur in HIV testing, in general, and HIV testing with those already affected by intimate partner violence (with or without a study). Some risks occurring with HIV testing, in general, are fears of someone finding out you have received an HIV test and/or feeling upset during the testing process, which can increase when someone is diagnosed as having HIV. When persons are diagnosed with HIV, they may feel fear of or actual isolation or judgment from their family and community. You may be at-risk of worse violence if there is already a history of partner violence in your relationship and because partner violence may increase when someone is either diagnosed with HIV or attempting to receive HIV treatment. Risks specific to this study are the possibility of feeling slightly uncomfortable when answering difficult questions about personal or sexual experiences; answering these questions may cause you to recall upsetting situations from the past. If this happens, you will be given information for a local agency that may assist you. Another potential risk, specific to this study, is that Shout-it-Now will use the telephone to make contact with you. This could be dangerous if your partner is aware that you have received this phone call. Do not answer the phone if it is not safe to do so. You may contact the call centre when it is safe or we will try to reach you at another time. Shout-in-Now has been using this call back approach for some time and no negative experiences have been reported. Also, research has shown that the use of telephone check-ins can help increase safety with women who have been harmed in their relationship. Therefore, the benefits of this study appear to be greater than the risks. 


\section{Benefits}

This study has many potential benefits. You may experience the feeling of being more supported or having more resources as a result of participating in this study. You may find that the questions help you to think about your relationship and seek out help from your family or community so that you are safer. For participants diagnosed with HIV, it is the hope that they will be able to get HIV treatment more safely than if they had not participated in this study. In general, the hope is that participants will experience feeling safer as a result of participating in this study. Research shows that some women, who participate in studies about partner violence, say they found the study to be beneficial to them. Additionally, information from this study will be used to help other women get HIV services in a safer manner. By getting HIV services more safely, women may feel more satisfied with their life as well experience increased healthiness or longer life. Ultimately, these experiences could lead to less people infecting others with HIV as well as less people dying from HIV-related death. Although information in this study may not benefit you directly, the information learned in this study may be helpful to others.

\section{Compensation}

If you participate in this study, and remain in the study throughout the entire course of the study, your name will be entered into a drawing for a free i-pod. Additionally, by participating in any part of this study, you will receive a free HIV/TB screening and counseling as well as a free intimate partner violence assessment. If you prefer not to participate in this study, you will still receive a free HIV/TB screening and counseling but no intimate partner violence screen.

\section{Confidentiality}

Total privacy cannot be guaranteed. Your privacy will be protected to the extent permitted by law. If the results from this study are published, your name will not be made public. While unlikely, the following may look at the study records:

The University of Louisville Institutional Review Board, Human Subjects Protection Program Office, and Privacy Office.

Office for Human Research Protections (OHRP)

Each case will receive a case identification number so that your name is not used to identify your information. Data (or information from this study) will be stored electronically by the research team in South Africa on a secure server. Once the study has been completed, study information, (data) in the form of case identification numbers and not names, will be sent by email to the primary investigator of this study. The study information (data) to be provided will be stored on the computer of the primary investigator. Access to the computer will be password protected, and the computer will be HIPAA compliant (follow privacy guidelines of the Health Insurance Portability and Accountability Act). A copy of 
original files will be saved on a CD and be stored in a locked cabinet in the primary investigator's office.

\section{Voluntary Participation}

Taking part in this study is voluntary. You may choose not to take part at all. If you decide to be in this study you may stop taking part at any time. If you decide not to be in this study or if you stop taking part at any time, you will not lose any benefits for which you may qualify.

\section{Research Subject's Rights, Questions, Concerns, and Complaints}

If you have any concerns or complaints about the study or the study staff, you have three options.

You may contact the principal investigator at 502-852-2430 (USA).

If you have any questions about your rights as a study subject, questions, concerns or complaints, you may call the Human Subjects Protection Program Office (HSPPO) (502) 852-5188. You may discuss any questions about your rights as a subject, in secret, with a member of the Institutional Review Board (IRB) or the HSPPO staff. The IRB is an independent committee composed of members of the University community, staff of the institutions, as well as lay members of the community not connected with these institutions. The IRB has reviewed this study.

If you want to speak to a person outside the University, you may call 1877-852-1167. You will be given the chance to talk about any questions, concerns or complaints in secret. This is a 24 hour hot line answered by people who do not work at the University of Louisville.

This paper tells you what will happen during the study if you choose to take part. Your signature means that this study has been discussed with you, that your questions have been answered, and that you will take part in the study. This informed consent document is not a contract. You are not giving up any legal rights by signing this informed consent document.

\section{Printed name of subject}

Telephone number of subject: 


\begin{tabular}{lrl}
\hline $\begin{array}{l}\text { Signature of Person Explaining the Consent Form } \\
\text { (if other than the Investigator) }\end{array}$ & & Date Signed \\
\hline Signature of Investigator & PHONE NUMBERS \\
LIST OF INVESTIGATORS & $1-502-852-2430$ & Date Signed \\
\multicolumn{2}{l}{$\begin{array}{l}\text { Michiel A. van Zyl, PhD } \\
\text { Lauren Brown, LCSW, PhD Candidate 1-502-852-2430 }\end{array}$}
\end{tabular}


Pre-test: Danger Indicator English

\section{Pre-test English SOC \& Exp Groups}

Script: "Hi, this is with Shout-it-Now. I was given your information from my shout-it-now teammate because you agreed to participate in a brief study with us. This will take about 20 minutes or more. Are you still willing to participate in this study? Great. Is this a safe time to talk? okay, I am going to ask you some questions now."

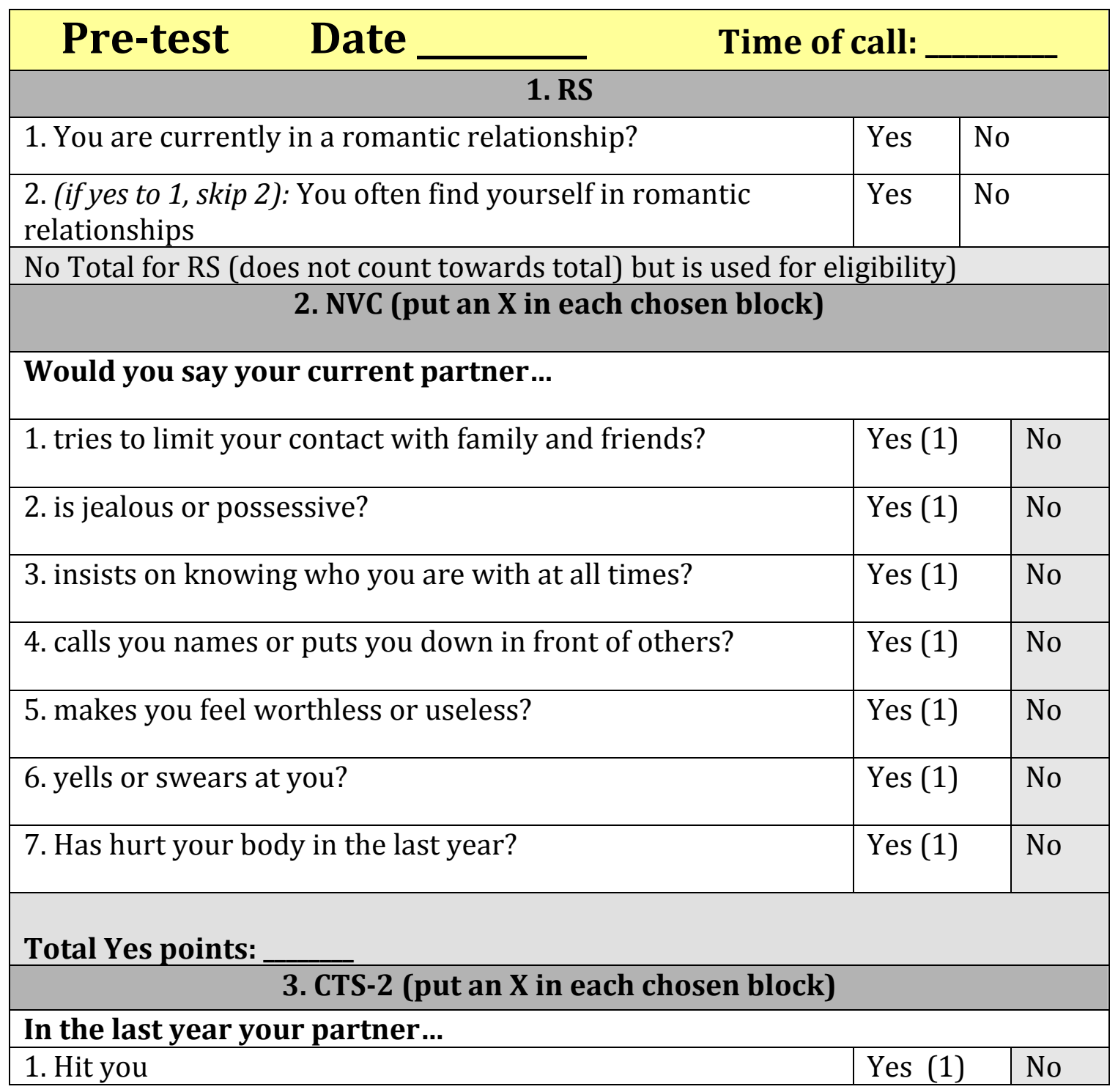




\begin{tabular}{|c|c|c|c|}
\hline 2. Hit you with something & Yes (1) & \multicolumn{2}{|l|}{ No } \\
\hline 3. Choked you & Yes (1) & No & \\
\hline 4. Beat you up & Yes (1) & No & \\
\hline 5. Threatened you with a weapon & Yes (1) & No & \\
\hline 6. Used a weapon on you & Yes (1) & No & \\
\hline 7. Made you have sex without a condom & Yes (1) & No & \\
\hline 8. Used force to make you have sex & Yes (1) & No & \\
\hline 9. Used force to make you have oral or anal sex & Yes (1) & No & \\
\hline \multicolumn{4}{|l|}{ Total Yes points: } \\
\hline \multicolumn{4}{|c|}{$\begin{array}{l}\text { To be IPV eligible: Must score } 2 \text { or higher on NVC or score } 1 \text { or higher in CTS- } \\
2 \begin{array}{ll}\text { NVC: } & \text { CTS-2: }\end{array} \\
\begin{array}{l}\text { Notal: }\end{array}\end{array}$} \\
\hline \multicolumn{4}{|c|}{$\begin{array}{l}\text { For those not IPV eligible: "Thank you very much for answering these } \\
\text { questions. We will not be in contact with you any further. Before I go, do you } \\
\text { mind if I quickly just ask what your age and race are for research purposes? } \\
\text { Age } \\
\text { Thank you." }\end{array}$} \\
\hline \multicolumn{4}{|c|}{ 4. VVS (Put an $X$ in each chosen block) } \\
\hline \multicolumn{4}{|l|}{$\begin{array}{l}\text { How frequent do the following happen: } \\
\text { Almost never or never } \\
\text { Frequently }\end{array}$} \\
\hline $\begin{array}{l}\text { 1. Your partner seems to view your words or actions more } \\
\text { negatively than you mean them to be. }\end{array}$ & 0 & 0 & 1 \\
\hline $\begin{array}{l}\text { 2. When you all have a problem to solve, it is like you are on } \\
\text { opposite teams. }\end{array}$ & 0 & 0 & 1 \\
\hline 3. You feel lonely in the relationship. & 0 & 0 & 1 \\
\hline $\begin{array}{l}\text { 4. When you argue, one of you withdraws ... that is, does not } \\
\text { want to talk about it anymore or leaves the scene. }\end{array}$ & 0 & 0 & 1 \\
\hline 5. You are afraid of your partner. & 0 & 0 & 1 \\
\hline $\begin{array}{l}\text { 6. You feel like your opinions or emotions do not matter in your } \\
\text { relationship. }\end{array}$ & 0 & 0 & 1 \\
\hline $\begin{array}{l}\text { 7. When you argue, your partner always has to "win" the } \\
\text { argument and will not really listen to your side of the story. }\end{array}$ & 0 & 0 & 1 \\
\hline $\begin{array}{l}\text { 8. You have been nervous or afraid to refuse your partner's } \\
\text { sexual advances. }\end{array}$ & 0 & 0 & 1 \\
\hline \multicolumn{4}{|l|}{ Total "Frequently" points: } \\
\hline 5. HIRS (put an X in each chosen box) & & & \\
\hline
\end{tabular}




\begin{tabular}{|c|c|c|}
\hline \multicolumn{3}{|l|}{$\begin{array}{l}\text { Do you agree with the following statements? } \\
\text { Because of your HIV+ status... }\end{array}$} \\
\hline 1. You will be in trouble with your partner & Yes (1) & No \\
\hline 2. Your partner will be a support to you & No (1) & $\begin{array}{l}\text { Yes } \\
(0)\end{array}$ \\
\hline 3. Your relationship with your partner will worsen & Yes (1) & No \\
\hline $\begin{array}{l}\text { 4. Receiving treatment will be dangerous because of your } \\
\text { partner }\end{array}$ & Yes (1) & No \\
\hline 5. You will feel unsafe in your relationship & Yes (1) & No \\
\hline 6. Others will help you if you feel unsafe in your relationship & No (1) & $\begin{array}{l}\text { Yes } \\
(0)\end{array}$ \\
\hline 7. It is not safe to discuss HIV testing with your partner & Yes (1) & No \\
\hline 8. It is not safe to discuss your HIV status with your partner & Yes (1) & No \\
\hline 9. You feel safe getting to medical appointments & No (1) & $\begin{array}{l}\text { Yes } \\
(0)\end{array}$ \\
\hline 10. You fear your partner will try to kill you & Yes (3) & No \\
\hline \multicolumn{3}{|c|}{$\begin{array}{l}\text { Total points: } \_ \text {(remember question } 10 \text { is worth } 3 \text { points and } 2,6 \& 9 \\
\text { are reverse scoring) }\end{array}$} \\
\hline \multicolumn{3}{|c|}{ 6. DA (put an $\mathrm{X}$ in each chosen block) } \\
\hline \multicolumn{3}{|l|}{ In the last year... } \\
\hline 1. Has the violence in your relation & Yes (1) & No \\
\hline 2. Does your partner own a gun? & Yes (2) & No \\
\hline 3. Have you left or broken up with your partner? & Yes (2) & $\begin{array}{l}\text { No } \\
(0)\end{array}$ \\
\hline 4. Does your partner threaten to kill you? & Yes (2) & No \\
\hline $\begin{array}{l}\text { 5. Does your partner use illegal drugs such as (e.g. tik, } \\
\text { crack/cocaine, sniff glue, etc.) or mix dagga with other drugs? }\end{array}$ & Yes (1) & No \\
\hline 6. Is your partner an alcoholic or problem drinker? & Yes (1) & No \\
\hline 7. Has your partner ever threatened to commit suicide? & Yes (2) & No \\
\hline $\begin{array}{l}\text { 8. Does your partner follow or spy on you or leave } \\
\text { threatening notes or messages? }\end{array}$ & $\begin{array}{l}\text { Yes (.5) } \\
\text { half }\end{array}$ & No \\
\hline 9. Has your partner destroyed your property? & $\begin{array}{l}\text { Yes (.5) } \\
\text { half }\end{array}$ & No \\
\hline 10. Have you ever threatened or tried to commit suicide? & Yes $(0)$ & $\begin{array}{l}\text { No } \\
(0)\end{array}$ \\
\hline 11. Do you have a child that is not by your current partner? & Yes (1) & $\begin{array}{l}\text { No } \\
(0)\end{array}$ \\
\hline \multicolumn{3}{|c|}{$\begin{array}{l}\text { Total points } \_ \text {(remembering that questions } 2,3,4, \& 7 \text { count } 2 \text { points } \\
\text { each): } \\
\text { Time of End of Pre-test }\end{array}$} \\
\hline
\end{tabular}


Appendix E

Initial Safety Plan

\section{Safety Plan Protocol: (Script): Time Safety plan begins:}

Based on your answers to questions that tell us how safe you are and feel in your relationship you have a score of out of 10 points where 10 means extremely high danger. How do you feel hearing this information? (2)

Your HIV diagnosis is new and you have not had much time to think about it. It may be difficult to think about much else right now, but it is important to start thinking about how you will stay safe in your relationship with this diagnosis.

I have some important information for you. Women experience violence in their relationships in three main ways: emotionally, physically and sexually. All of these types of abuse may increase after an HIV diagnosis. Examples of emotional abuse are name-calling, putting you down or trying to control you. Examples of physical abuse are hitting you, kicking you, or slapping you. Examples of sexual abuse are forcing you to have sex or forcing you to have sex without a condom.

Women, who are in a relationship where there is abuse or violence, are at an increased risk for more violence after they receive an HIV diagnosis. Some women experience more danger when they disclose their HIV status to their partner or when they attempt to get medical care or medication for HIV. Abusive partners may try to prevent them from accessing treatment or from taking medication.

Although it can be a dangerous time in your relationship, many women prefer to tell their partner about their status so that their partner may also get tested or get treatment. If you decide to tell your partner, it is important to come up with a plan for the safest way to do so. It is your decision if you decide you want to tell your partner about your HIV status.

Is it okay if we briefly talk about some ways to stay safe in your relationship?

1. There are many people in your life and community that can help you if you feel you are in danger in your relationship. This could include your doctor, nurse, counselor, clergy or spiritual leader or a service specifically for relationship abuse.

$>$ There are free and confidential hotlines you can call if you need someone to talk to or emergency shelter. One number is: 0800-150150. It is important to write this in a safe place so that your partner cannot find it. Where are some safe places you could put this? (ex: work or in your phone as a different listing).

(3)

2. Some people prefer to talk with or get help from people they know. This may mean talking with your family or friends or someone in your community. Talking with a family member or friend can help you if an emergency occurs. 
Think about which of your family members or friends are safe (will not relay information to your partner). You may call this person if there is an emergency (and use a code word to signal for help).

3. There is also legal help for partner violence. Some examples are calling the police, contacting a lawyer or filing for an order of protection.

$>$ Basically, an order of protection will prevent your partner from entering your home or your work, or from having contact with your children. Also, these orders help prevent further abusive acts. Would you like the number to access information about an order of protection? (must be completed via application or affidavit with local police department) or through head office (in Pretoria) at +27 (0) 12 3931000.

$>$ Keep this in a safe place (family member's house, on you at home or in your purse in a secret place like inside tube of lipstick or write the name of a different service next to it (hair salon, take out food, etc)

4. Some people try to do things to avoid abuse during dangerous times. When fighting occurs, some people attempt to calm their partner down, but remember his/her behavior is not your fault or responsibility.

$>$ Are there any particular times you can think of you might want to stay clear because it is most dangerous? (4)

5. Some people try to resist the abuse when it occurs. Examples of this are fighting back, hiding a gun where only you know where it is, trying to leave or end the relationship, or running and hiding during episodes. Research has shown that resisting the abuse can be more dangerous and increase the violence.

$>$ Are you considering leaving your relationship? (5) Yes No If so, it is good to create a plan because this is the most dangerous time in a violent relationship. (6) Plan is

6. Other strategies include preparing for an emergency and hiding money, important phone numbers and personal items in case you need to leave in a hurry. It is also good to plan in advance where you could go in your house if a fight occurs (go to a safe room where there are no weapons or sharp objects)

In closing this call, I would like to help you make a plan for how you may safely take care of yourself, given this new diagnosis and the danger in your relationship.

If you decide to tell your partner about your status, now or sometime later, what are some ways you can protect yourself? (7)

If you suspect your partner is having a sexual relationship with someone else, it is important to know that confronting your partner about this may lead to more violence. Using condoms, after getting an HIV diagnosis, has been shown to reduce re-infection but asking your partner to use condoms has also been shown to 
increase violence. If you're going to begin using condoms, consider the safest way to talk to your partner about this.

It is best after a diagnosis to immediately go to a clinic to get medical care. Until you decide if you want to tell your partner about the diagnosis, how can you safely get medical services? Do you need to plan to tell your partner you are going somewhere else? What could you say? (8)

If your CD4 cell count is low (less than 500), it will be recommended that you begin antiretroviral therapy medication. A common safety strategy is to hide these medications from an abusive partner. What are some ways you could do this if needed? (for example, putting pills into a Tylenol bottle and/or keeping it with you). What can you do? (9)

Is there anything else we can help you with today so that you can safely get the medical attention you need and take care of yourself physically and emotionally? (10)

\section{Time safety plan ends}

Qualitative answers from Safety Plan:

1. Level of danger is:

2. Feelings after hearing level of danger:

3. If accepted free hotline number, where is safe place to keep number:

4. Times/situations need to stay away from partner:

5. Yes or No (to leaving relationship)

6. Plan for leaving is:

7. Ways to protect self if discloses to partner:

8. Plan to safely get to HIV medical care or clinic:

9. Plan to keep HIV medication safe (if applicable):

10. Anything else we can help with to keep physically and emotionally safe: 
Appendix F

\section{Initial safety plan for past testers}

\section{Safety Plan Protocol Past Testers: (Script): Time Safety plan begins:}

Based on your answers to questions that tell us how safe you are and feel in your relationship you have a score of out of 10 points where 10 means extremely high danger. How do you feel hearing this information? (2)

Given that you have known about your HIV status for some time, can you tell us what caused you to return to Shout-it-Now recently to be retested? 3)

Although you have had some time to adjust to this diagnosis, your relationship may be affected by your HIV status or your need to get treatment.

I have some important information for you. Women experience violence in their relationships in three main ways: emotionally, physically and sexually. All of these types of abuse may increase after an HIV diagnosis. Examples of emotional abuse are name-calling, putting you down or trying to control you. Examples of physical abuse are hitting you, kicking you, or slapping you. Examples of sexual abuse are forcing you to have sex or forcing you to have sex without a condom.

Women, who are in a relationship where there is abuse or violence, are at an increased risk for more violence once they are diagnosed with HIV. Some women experience more danger when they disclose their HIV status to their partner or when they attempt to get medical care or medication for HIV. Abusive partners may try to prevent them from accessing treatment or from taking medication.

Have you experienced any difficulties getting to your HIV appointments or being able to safely take your medication because of your relationship? 4) A) No (B)Yes: explain:

A) No: If you have not experienced danger in your relationship related to getting to medical appointments or taking HIV medication, can you talk about how you approached this with your partner that allowed these activities to be safe?

Thank you. Do you mind if I tell you about some other general strategies for staying safe when there is violence in your relationship and you must take care of your health? (follow with safety plan below)

B) Yes: Can we discuss a plan for you to do these things safely? (follow with safety plan below)

7. There are many people in your life and community that can help you if you feel you are in danger in your relationship. This could include your doctor, nurse, counselor, clergy or spiritual leader or a service specifically for relationship abuse. 
There are free and confidential hotlines you can call if you need someone to talk to or emergency shelter. One number is: 0800-150150. It is important to write this in a safe place so that your partner cannot find it. Where are some safe places you could put this? (ex: work or in your phone as a different listing).

(5)

8. Some people prefer to talk with or get help from people they know. This may mean talking with your family or friends or someone in your community. Talking with a family member or friend can help you if an emergency occurs.

$>$ Think about which of your family members or friends are safe (will not relay information to your partner). You may call this person if there is an emergency (and use a code word to signal for help).

9. There is also legal help for partner violence. Some examples are calling the police, contacting a lawyer or filing for an order of protection.

> Basically, an order of protection will prevent your partner from entering your home or your work, or from having contact with your children. Also, these orders help prevent further abusive acts. Would you like the number to access information about an order of protection? (must be completed via application or affidavit with local police department) or through head office (in Pretoria) at +27 (0) 12 3931000.

$>$ Keep this in a safe place (family member's house, on you at home or in your purse in a secret place like inside tube of lipstick or write the name of a different service next to it (hair salon, take out food, etc)

10. Some people try to do things to avoid abuse during dangerous times. When fighting occurs, some people attempt to calm their partner down, but remember his/her behavior is not your fault or responsibility.

$>$ Are there any particular times you can think of you might want to stay clear because it is most dangerous? (6)

11. Some people try to resist the abuse when it occurs. Examples of this are fighting back, hiding a gun where only you know where it is, trying to leave or end the relationship, or running and hiding during episodes. Research has shown that resisting the abuse can be more dangerous and increase the violence.

> Are you considering leaving your relationship? (7) Yes No If so, it is good to create a plan because this is the most dangerous time in a violent relationship. (8) Plan is

12. Other strategies include preparing for an emergency and hiding money, important phone numbers and personal items in case you need to leave in a hurry. It is also good to plan in advance where you could go in your house if a fight occurs (go to a safe room where there are no weapons or sharp objects) 
In closing this call, I would like to help you make a plan for how you may safely take care of yourself, given your on-going HIV care needed and the danger in your relationship.

When you need to get to medical appointments, now or sometime later, what are some ways you can protect yourself? (9)

If you suspect your partner is having a sexual relationship with someone else, it is important to know that confronting your partner about this may lead to more violence. Using condoms has been shown to reduce re-infection but asking your partner to use condoms has also been shown to increase violence. If you're going to use condoms, consider the safest way to talk to your partner about this.

If your CD4 cell count is low (less than 500), it will be recommended that you begin antiretroviral therapy medication. If it is unsafe for you partner to know you are taking these medications, a common safety strategy is to hide these medications from an abusive partner. What are some ways you could do this if needed? (for example, putting pills into a Tylenol bottle and/or keeping it with you). What can you do? (10)

Is there anything else we can help you with today so that you can safely get the medical attention you need and take care of yourself physically and emotionally? (11) 


\section{Follow-up Safety plan}

\author{
Appendix G \\ Follow-up safety plan: \\ Time call begins
}

Directions:

First, before phone call review information discussed during first safety plan.

Then ask participant:

How has your relationship been in the last two weeks?

Have you been able to use the safety plan we created?

Can we review it together? (review plan from participant packet)

Has anything changed with your safety?

Do you need to discuss any new plans to increase your safety now?

I will be in touch with you one final time in about two weeks.

Thank you for talking with me.

\section{Time call ends:}


Appendix $\mathrm{H}$

Standard of Care Post-test

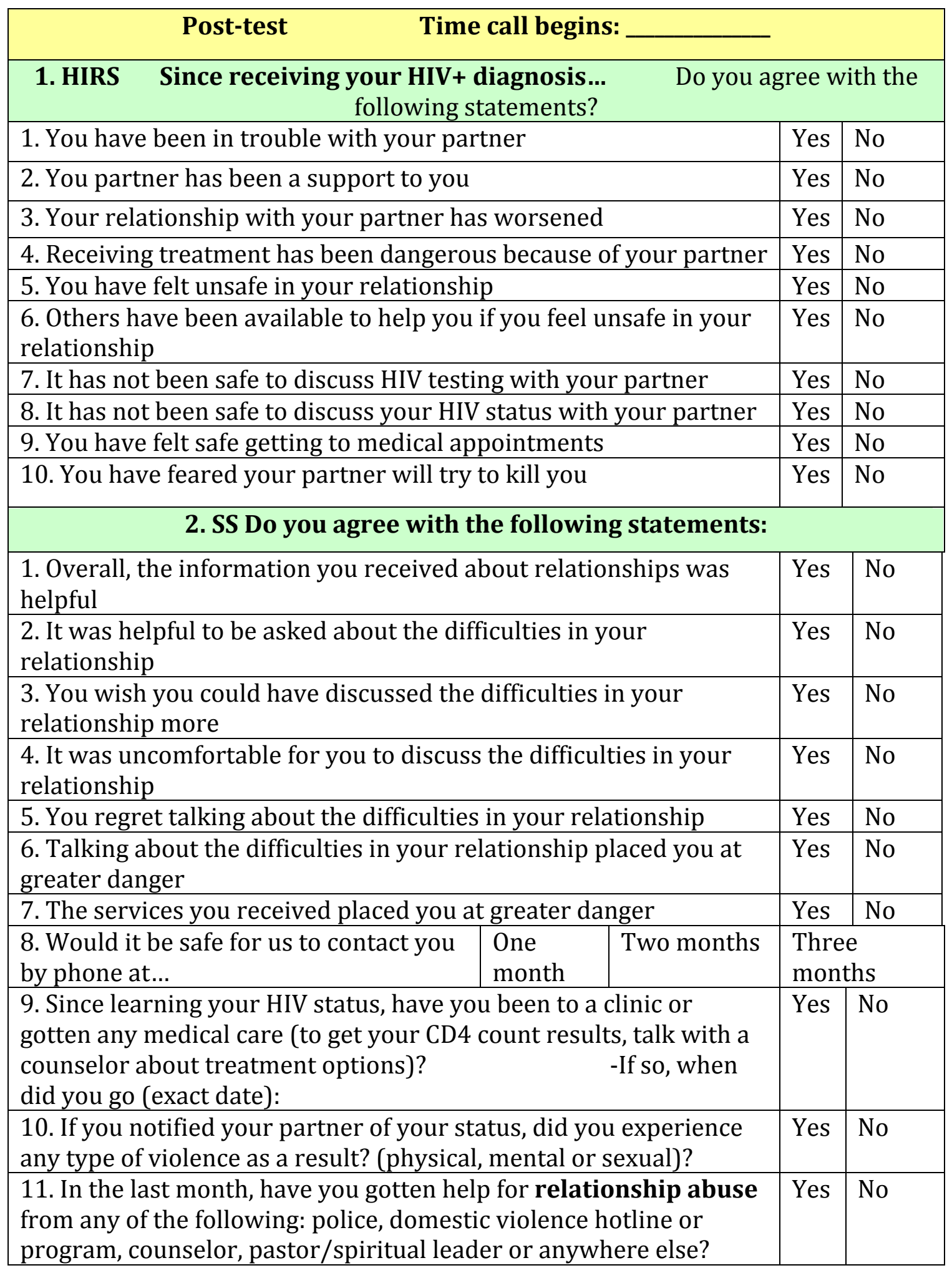


Appendix I

Experimental Post-test

\begin{tabular}{|c|c|c|c|c|c|}
\hline \multicolumn{5}{|c|}{ Time call begins: } & \\
\hline \multicolumn{6}{|c|}{ 1. HIRS } \\
\hline $\begin{array}{l}\text { Since receiving your HIV+ diagnosis } \\
\text { statements? }\end{array}$ & \multicolumn{5}{|c|}{ Do you agree with the following } \\
\hline \multicolumn{2}{|c|}{ 1. You have been in trouble with your partner } & \multicolumn{3}{|l|}{ Yes } & No \\
\hline \multicolumn{2}{|l|}{ 2. Your partner has been a support to you } & \multicolumn{3}{|l|}{ Yes } & No \\
\hline \multicolumn{2}{|c|}{ 3. Your relationship with your partner has worsened } & \multicolumn{3}{|l|}{ Yes } & No \\
\hline \multicolumn{2}{|c|}{$\begin{array}{l}\text { 4. Receiving treatment has been dangerous because of your } \\
\text { partner }\end{array}$} & \multicolumn{3}{|l|}{ Yes } & No \\
\hline \multicolumn{2}{|c|}{ 5. You have felt unsafe in your relationship } & \multicolumn{3}{|l|}{ Yes } & No \\
\hline \multicolumn{2}{|c|}{$\begin{array}{l}\text { 6. Others have been available to help you if you feel unsafe in } \\
\text { your relationship }\end{array}$} & \multicolumn{3}{|l|}{ Yes } & No \\
\hline \multicolumn{2}{|c|}{ 7. It has not been safe to discuss HIV testing with your partner } & \multicolumn{3}{|l|}{ Yes } & No \\
\hline \multicolumn{2}{|c|}{$\begin{array}{l}\text { 8. It has not been safe to discuss your HIV status with your } \\
\text { partner }\end{array}$} & \multicolumn{3}{|l|}{ Yes } & No \\
\hline \multicolumn{2}{|c|}{ 9. You have felt safe getting to medical appointments } & \multicolumn{3}{|l|}{ Yes } & No \\
\hline \multicolumn{2}{|c|}{ 10. You have feared your partner will try to kill you } & \multicolumn{3}{|l|}{ Yes } & No \\
\hline \multicolumn{6}{|c|}{ 2.SS } \\
\hline \multicolumn{6}{|l|}{ Do you agree with the following: } \\
\hline \multicolumn{2}{|c|}{$\begin{array}{l}\text { 1. Overall, the information you received about relationships was } \\
\text { helpful }\end{array}$} & & Yes & No & \\
\hline \multicolumn{2}{|c|}{$\begin{array}{l}\text { 2. It was helpful to be asked about the difficulties in your } \\
\text { relationship }\end{array}$} & & Yes & No & \\
\hline \multicolumn{2}{|c|}{$\begin{array}{l}\text { 3. You wish you could have discussed the difficulties in your } \\
\text { relationshin more }\end{array}$} & & Yes & No & \\
\hline \multicolumn{2}{|c|}{$\begin{array}{l}\text { 4. It was uncomfortable for you to discuss the difficulties in your } \\
\text { relationship }\end{array}$} & & Yes & No & \\
\hline \multicolumn{2}{|c|}{ 5. You regret talking about the difficulties in your relationship } & & Yes & No & \\
\hline \multicolumn{2}{|c|}{$\begin{array}{l}\text { 6. Talking about the difficulties in your relationship placed you at } \\
\text { greater danger }\end{array}$} & & Yes & No & \\
\hline \multicolumn{2}{|c|}{ 7. The services you received placed you at greater danger } & & Yes & No & \\
\hline & & & & & \\
\hline 1. Did you use the safety plan? & Yes & & No & & \\
\hline 2. Did you find the safety plan helpful? & Yes & & No & & \\
\hline strategies & stly use? & & & & \\
\hline A. Talking to doctor, nurse or other $h$ & ofessional & & & Yes & \\
\hline B. Talking to counselor & & & & Yes & \\
\hline
\end{tabular}




\begin{tabular}{|c|c|c|c|c|c|}
\hline \multicolumn{4}{|c|}{ C. Talking to a pastor, spiritual leader or someone at church } & Ye: & No \\
\hline \multicolumn{4}{|c|}{$\begin{array}{l}\text { D. Seeking help with a domestic violence service (in-person or by phone; } \\
\text { individual or group) }\end{array}$} & Ye & No \\
\hline \multicolumn{4}{|c|}{ E. Talking to Family or friends } & Ye: & No \\
\hline \multicolumn{4}{|c|}{$\begin{array}{l}\text { F. Contacting police, attorney or getting an order of } \\
\text { protection/restraining order, court order or interdict }\end{array}$} & Ye: & No \\
\hline \multicolumn{4}{|c|}{$\begin{array}{l}\text { G. Trying to avoid the violence (activities like avoiding partner at certain } \\
\text { times, doing whatever partner wants you to do, trying to keep things } \\
\text { quiet or a secret from partner) }\end{array}$} & Ye: & Nc \\
\hline \multicolumn{4}{|c|}{$\begin{array}{l}\text { H. Fighting back, trying to end the relationship, running and hiding when } \\
\text { violence occurs }\end{array}$} & Ye: & $\mathrm{Nc}$ \\
\hline \multicolumn{4}{|c|}{$\begin{array}{l}\text { I. Thinking ahead and doing things like hiding money, keeping important } \\
\text { phone numbers with you or other behaviors to help prepare for an } \\
\text { emergency }\end{array}$} & Ye: & $\mathrm{Nc}$ \\
\hline \multicolumn{4}{|c|}{$\begin{array}{l}\text { J. Creating a plan around the safest way to get to a clinic or medical care } \\
\text { for HIV treatment }\end{array}$} & Ye: & $\mathrm{Nc}$ \\
\hline \multicolumn{4}{|c|}{$\begin{array}{l}\text { K. Creating a plan around the safest way to get medication or safely take } \\
\text { medication regularly. }\end{array}$} & Ye & $\mathrm{Nc}$ \\
\hline $\begin{array}{l}\text { 4. Would you recommend a safety plan for } \\
\text { friends in similar situations? }\end{array}$ & Yes & \multicolumn{3}{|c|}{ No } & \\
\hline $\begin{array}{l}\text { 5. Would it be safe for us to contact you by } \\
\text { phone at... }\end{array}$ & \begin{tabular}{|l|} 
One \\
mont \\
h
\end{tabular} & $\begin{array}{l}\text { Two } \\
\text { month } \\
\text { s }\end{array}$ & $\begin{array}{l}\text { Three } \\
\text { months }\end{array}$ & & \\
\hline \multicolumn{2}{|c|}{$\begin{array}{l}\text { 6. Since learning your HIV status, have you been to a } \\
\text { clinic or gotten any medical care (to get your CD4 count } \\
\text { results, talk with a counselor about treatment options)? } \\
\text { If so, when did you go (exact date): }\end{array}$} & \multicolumn{3}{|l|}{ Yes } & \\
\hline \multicolumn{2}{|c|}{$\begin{array}{l}\text { 7. If you notified your partner of your status, did you } \\
\text { experience any type of violence as a result? (physical, } \\
\text { mental or sexual)? }\end{array}$} & Yes & \multicolumn{2}{|c|}{ No } & \\
\hline
\end{tabular}


Appendix J

\title{
Informed Consent isiXhosa
}

\author{
UXwebhu lokuNikezelwa kwemVume ngoLwazi \\ UkuHlolwa kwe-HIV noPhononongo koKhuseleko lobuNdlobongela \\ IweQabane eliseNyongweni
}

Igama nedilesi yom/abaXhasi: IYunivesithi yaseLouisville, eLouisville, KY kunye ne-Shout-it-Now, eKapa, eMzantsi Afrika

Igama nedilesi yom/abaphandi: Umphandi oyiNtloko uMichiel A. van Zyl noGxa wakhe okwangumphandi uLeslie Lauren Brown: 2217 South Third St. Louisville, KY 40292, eUSA.

I/iindawo oluza kuqhutywa kuzo uphononongo: Kwiphondo laseGauteng yaye iminxeba iza kwenziwa eKapa, eMzantsi Afrika.

linombolo zeminxeba abafanele batsalele kuzo abalingwa xa benemibuzo: 021 7134414

\section{InTshayelelo nenkCazelo engentSusa}

Uyamenwa ukuba ube nenxaxheba kuphononongo lophando. Olu phononongo luqhutywa nguGqr. uRiaan van Zyl, PhD; uLauren Brown, LCSW, ogaqele iPhD kunye ne-Shout-it-Now. Olu phononongo luxhaswa yiYunivesithi yaseLouisville, iSebe leSikolo iKent sezeNtlalo-ntle. Olu phononongo luza kuqhutywa kuzo zonke indawo ezingoomahamba-ehlala zeengCebiso nezokuHlola i-HIV kwiphondo laseGauteng ezityelelwa yi-S-N ngexesha lokuqhutywa kolu phononongo. Uqhagamshelwano malunga nolu phononongo luza kuqhutywa ngomnxeba, kwiziko leminxeba le-Shout-it-Now, eliseTokai, eNtshona Koloni. Ngabalingwa abamalunga nama-225 abaza kumenywa ukuba babe nenxaxheba.

\section{Injongo}

Injongo yolu phononongo kukubona enobana abantu basetyhini bayaxhamla na ngokwazi amanqaku angokhuseleko kwabo bancuma nabo xa behlolelwa i-HIV nokuthi banaso na isicwangciso sokhuseleko emveni kokuba behloliwe.

\section{linkQubo}

Kolu phononongo, uza kunxityelelwana nawe ngomnxeba emva kosuku ufumene iinkonzo ze-Shout-it-Now. Uthatho-nxaxheba lusenokwenzeka kwesinye sezi zigaba zimbini. 
Ukuba uza kuthatha inxaxheba kwisiGaba I solu phononongo, udliwano-ndlebe lomnxeba okanye kwiqela elithile luya kusetyenziselwa ukuphucula uphando lophononongo okanye ungenelelo kuphela (umba wencoko yomnxeba). Siza kuqhagamshelana nawe ukuze sive uluvo Iwakho ngemibuzo yophando olo kunye nomba wencoko yomnxeba. Sifuna ukwazi enobana ucinga ukuba imibuzo iluncedo na, enobana ikhona na into onokuyivuna kuyo, ikwenza ukhululeke okanye enobana kufuneke kwenziwe iinguqu ezithile na ukuze abantu bayiqonde imibuzo okanye ingxoxo baze bazive bexolile yile nkqubo. Eminye okanye yonke imibuzo ekwisiGaba II usenokuyibuzwa kwesi sigaba. Olu dliwano-ndlebe okanye amagqela akazokurekhodwa, kunoko, ingxelo yakho iya kubhalwa ngesandla okanye ichwethezwe ekhompyutheni.

Ukuba uza kuthatha inxaxheba kwisiGaba II solu phononongo, uza kubuzwa imibuzo ngenjongo yokufumanisa ukuba ubudlelwane bakho bukhuseleke kangakanani kubundlobongela. Eminye yemibuzo yenzelwe olu phononongo, ize eminye imibuzo ifane naleyo idla ngokubuzwa kwiindawo ngeendawo zehlabathi ngenjongo yokufumanisa ukhuseleko kubudlelwane. Eminye imibuzo ihlobene nobundlobongela ngokwasemzimbeni nangokwesondo besinqanda-mathe sakho kwaye eminye ihlobene nokhuseleko ngokwasengqondweni. Kukwakho nemibuzo embalwa engamava osenokuba wawukhe wanawo ngamaqabane anobundlobongela kwanendlela ocinga ngayo ngokumayela nobundlobongela kubudlelwane. Kuya kuqhagamshelwana nawe izihlandlo ezi-2 ukuya kwezi-3 kolu phononongo uze ubuze le mibuzo kunxibelelwano lokuqala nolokugqibela. Imibuzo yokuqala, iyonke, ifanele ithathe imizuzu esi-8 ukuya kwengama-20 ukuyiphendula. Kukho imibuzo emifutshane engama-46 kunxibelelwano lokuqala nemibuzo emifutshane eli-18 ukuya kwengama-30 kunxibelelwano lokugqibela. Xa kunokwenzeka ukuba nayiphi na imibuzo ikwenze ungakhululeki, usengakhetha ukungayiphenduli. Uya kuqhubeka ufumana iinkonzo ze-S-N kwanokuba akuyiphenduli yonke okanye eminye yale mibuzo. Abathathi-nxaxheba besiGaba II solu phononongo baya kufakwa kumaqela awohlukeneyo ophononongo ukuze abathathi-nxaxheba bangafumani iinkonzo ezifanayo bonke.

\section{ImiNgcipheko eKhoyo}

Olu phononongo ngokunokwalo alunamingcipheko ekhoyo, kodwa inkoliso yemingcipheko yenzeka xa kuhlolwa i-HIV ngokubanzi, naxa kuhlolwa i-HIV kwabo bachatshazelwa lugonyamelo Iwezinqanda-mathe zabo (abakolu phononongo okanye abangekho kulo). Eminye imingcipheko eyenzekayo xa kuhlolwa i-HIV ngokubanzi, luloyiko lokuba omnye umntu usenokufumanisa ukuba uhlolwe i-HIV kunye/okanye nokukhathazeka xa uhlolwa, yaye kungade kuthi chatha xa umntu kufunyaniswe ukuba une-HIV, usenokoyika ukubukulwa okanye ukugxekwa lusapho Iwakhe naluluntu. Usenokuba semngciphekweni wobundlobongela obumasikizi xa ngaba iqabane lakho belikhe lanobundlobongela kubudlelwane benu yaye ngenxa yokuba ubundlobongela beqabane busenokuthi chatha xa omnye efunyaniswe ene-HIV okanye ezama ukufumana unyango Iwe-HIV. Imingcipheko yolu phononongo kukuziva 
unendawana engakhululekanga xa uphendula imibuzo enzima engamava obuqu okanye ezesondo; ukuphendula le mibuzo kungakuvuselela iinkumbulo ezingamnandanga ezadlulayo. Ukuba kwenzeka loo nto, uya kunikwa inkcazelo emalunga ne-arhente yasekuhlaleni engakunceda. Omnye umngcipheko okhoyo wolu phononongo ngowokuba i-Shout-it-Now iya kuqhagamshelana nawe ngomnxeba. Le nto ingayingozi xa iqabane lakho lisazi ukuba ufumene lo mnxeba. Ungawuphenduli umnxeba ukuba akukhuselekanga ukuwuphendula. Ungaqhagamshelana neziko leminxeba xa kukhuselekile ukwenjenjalo okanye siya kuzama ukuqhagamshelana nawe ngelinye ixesha. I-Shout-in-Now sele inexesha isebenzisa le ndlela yokutsala umnxeba yaye akukho mava mabi athe axelwa. Kwakhona, uphando lubonise ukuba ukusetyenziswa komnxeba kunokunceda ekwandiseni ukhuseleko kubantu basetyhini abathe bonzakala kubudlelwane babo. Ngoko ke, inzuzo zolu phononongo zibonakala zithe xhaxhe kunemingcipheko.

\section{linzuzo}

Olu phononongo luneenzuzo ezininzi. Unganemvakalelo yokuziva uxhaswa ngakumbi okanye unemithombo yoncedo engakumbi ngenxa yokuthatha inxaxheba kolu phononongo. Usengafumanisa ukuba le mibuzo ikunceda ucinge ngobudlelwane okubo uze ufune uncedo losapho Iwakho okanye loluntu ukuze ukhuseleke ngakumbi. Kubathathi-nxaxheba abafunyaniswe bene-HIV, kuthenjwa ukuba baya kufumana unyango Iwe-HIV ngokukhuseleke ngakumbi kunaxa bebengayi kuthatha inxaxheba kolu phononongo. Ngokubanzi, kuthenjwa ukuba abathathi-nxaxheba baya kuziva bekhuseleke ngakumbi ngenxa yokuthatha inxaxheba kolu phononongo. Uphando lubonisa ukuba abanye abantu basetyhini abathatha inxaxheba kolu phononongo malunga nogonyamelo Iwamaqabane abo, bathi bafumanise olu phononongo luluncedo kubo. Ukongezelela koko, inkcazelo yolu phononongo iya kusetyenziselwa ukunceda abanye abantu basetyhini ekufumaneni iinkonzo ze-HIV ngokukhuselekileyo. Xa befumana iinkonzo ze-HIV ngokukhuseleke ngakumbi, abasetyhini bayoneliseka ngakumbi ngobomi babo baze babe sempilweni ngakumbi babe nobomi obude. Ekugqibeleni, la mava angabangela abantu bangosulelani kangako nge-HIV yaye babe mbalwa nabantu ababulawa yi-HIV. Nangona inkcazelo ekolu phononongo isenokungabi yinzuzo kuwe ngokuthe ngqo, inkcazelo efumaneke kolu phononongo ingabanceda abanye.

\section{Imbuyekezo}

Ukuba uthatha inxaxheba kolu phononongo, uze uhlale kolu phononongo de luphele, igama lakho liya kuluhlu Iwamagama aza kutsalelwa ukufumana i-i-pod yasimahla. Ukongezelela, ngokuthatha inxaxheba kulo naliphi na icandelo lolu phononongo, uya kuhlolelwa i-HIV/TB nokufumana ingcebiso simahla kunye nokuphononongelwa ubundlobongela beqabane lakho simahla. Ukuba ukhetha ukungathathi inxaxheba kolu phononongo, usaya kuqhubeka ufumana ukuhlolwa i-HIV/TB nokufumana iingcebiso simahla kodwa ungaphononongelwa ubundlobongela beqabane lakho. 


\section{Imfihlo}

Asinakukwazi ukuqinisekisa ukuba izinto ziya kuba yindaba yakwamkhozi ngokupheleleyo. Izinto zakho ziya kukhuselwa kangangoko kwamkelekileyo ngokwasemthethweni. Ukuba iziphumo zolu phononongo ziyapapashwa, igama lakho aliyi kuthiwa pahaha. Ngeli lixa kungenakufane kwenzeke, la maziko alandelayo asenokuzijonga iingxelo zophononongo:

IBhodi yokuHlola yeZiko leYunivesithi yaseLouisville, i-Ofisi yenkQubo yokuKhuselwa kwabaLingwa abangabaNtu, kwane-Ofisi yeemFihlo. I-Ofisi yokuKhuselwa koPhando IwabaNtu (OHRP)

Imeko nganye iya kufumana inombolo eyahlulayo ukuze igama lakho lingasetyenziselwa ukwahlula inkcazelo yakho. lingcombolo (okanye inkcazelo yolu phononongo) ziya kugcinwa ekhompyutheni liqela lophando eMzantsi Afrika kwiseva ekhuselekileyo. Lusakube lugqityiwe uphononongo, inkcazelo yophononongo, (iingcombolo) ebhalwe iinombolo hayi amagama, iya kuthunyelwa nge-imeyili kumphandi oyintloko wolu phononongo. Inkcazelo yophononongo (iingcombolo) eza kunikezelwa iya kugcinwa ekhompyutheni yomphandi oyintloko. Umntu ungena ngephasiwedi kuloo khompyutha, yaye loo khompyutha ilungiselelwe ihambisana ne-HIPAA (imigaqo yeemfihlo yoMthetho we-Inshorensi wokuPhatha nokuPhendulisa ngezeMpilo). Ikopi yeefayile zokuqala ziya kugcinwa kwi-CD zize zifakwe kwikhabhathi etshixwayo kwi-ofisi yomphandi oyintloko.

\section{UkuThatha iNxaxheba ngokuziThandela}

Uthatha inxaxheba kolu phononongo ngokuzithandela. Usenokukhetha ukungathathi inxaxheba kwaphela. Ukuba ukhetha ukuba kolu phononongo usenokuyeka ukuthatha inxaxheba nanini na. Ukuba ukhetha ukungabi nanxaxheba kolu phononongo okanye ukuba uyayeka nangaliphi na ixesha, soze uphulukane neenzuzo onokuzifumana.

\section{AmaLungelo, imiBuzo, amaXhala, kwaneziKhalazo zabaLingwa boPhando}

Ukuba unawo nawaphi na amaxhala okanye izikhalazo ngolu phononongo okanye ngabasebenzi bophononongo, ungakhetha kwezi zinto zintathu. (eUSA).

Ungaqhagamshelana nomphandi oyintloko kule nombolo 502-852-2430

Ukuba unayo nayiphi na imibuzo ngamalungelo akho njengomlingwa wophononongo, ngemibuzo, ngamaxhala okanye izikhalazo onazo, ungatsalela umnxeba kwi-Ofisi yenkQubo yokuKhuselwa koPhando IwabaNtu (HSPPO) (502) 852-5188. Ungaxoxa ngayo nayiphi na imibuzo engamalungelo akho njengomlingwa emfihlekweni, kunye nelungu leBhodi 
yokuHlola yeZiko (IRB) okanye umsebenzi we-HSPPO. I-IRB yikomiti ezimeleyo eyilwe ngamalungu ekomiti yeYunivesithi, abasebenzi bamaziko lawo, kunye namalungu oluntu angenanto yakwenza nala maziko. I-IRB iye yaluhlola olu phononongo.

Ukuba ufuna ukuthetha nomntu ongengoweYunivesithi, ungatsalela umnxeba apha 1-877-852-1167. Uya kunikwa ithuba lokuthetha ngayo nayiphi na imibuzo, amaxhala okanye izikhalazo onazo emfihlekweni. Lo ngumnxeba oxakekileyo weeyure ezingama-24 ophendulwa ngabantu abangasebenzeli iYunivesithi yaseLouisville.

Eli phepha likuxelela into eya kwenzeka ebudeni bophononongo ukuba ukhetha ukuthatha inxaxheba. Utyikityo Iwakho luthetha ukuthi olu phononongo luye Iwaxoxwa nawe, nokuthi imibuzo yakho iphendulwe, nokuthi uza kuthatha inxaxheba kolu phononongo. Olu xwebhu lokunika imvume unolwazi alusosivumelwano. Awunikezeli ngalo naliphi na ilungelo lakho elisemthethweni ngokutyikitya olu xwebhu lokunika imvume unolwazi.

Igama eliprintiweyo lomlingwa

English
Language preference:

isiZulu Xhosa

Sesotho

Inombolo yomnxeba yomlingwa:

Utyikityo lomLingwa/uMmeli osemThethweni

Umhla wokutyikitya

Utyikityo lomNtu obeCacisa iFomu yemVume (ukuba asinguye umPhandi)

Umhla wokutyikitya

Utyikityo lomPhandi

Umhla wokutyikitya

ULUHLU LWABAPHANDI

IINOMBOLO ZEMINXEBA

UMichiel A. van Zyl, PhD $1-502-852-2430$

ULauren Brown, LCSW, oGaqele iPhD 1-502-852-2430 
Pre-test: Danger Indicator isiXhosa

Xhosa Pre-test Exp \& SOC Groups Uvavanyo lokuQala Date ___ Time of call 1. RS

\begin{tabular}{|l|l|l|}
\hline 1. Ingaba uyathandana sithethanje? & Ewe & Hayi \\
\hline 2. (ukuba uthi Ewe ku-1, tsiba 2): Usoloko uzifumana uthandana & Ewe & Hayi \\
\hline \multicolumn{2}{|c|}{ No Total for RS (does not count towards total) but is used for eligibility) } \\
\hline \multicolumn{2}{|c|}{ 2. NVC (beka u-X kwibhloko nganye oyikhethileyo) } \\
\hline
\end{tabular}

\section{Ungatsho na ukuba iqabane lakho onalo ngoku...}

\begin{tabular}{|l|l|l|}
\hline $\begin{array}{l}\text { 1. lizama ukunciphisa unxibelelwano lwakho nosapho lwakho } \\
\text { kwanabahlobo? }\end{array}$ & $\begin{array}{l}\text { Ewe } \\
(1)\end{array}$ & Hayi \\
\hline 2. linesikhwele okanye liyalinda? & $\begin{array}{l}\text { Ewe } \\
(1)\end{array}$ & Hayi \\
\hline $\begin{array}{l}\text { 3. lisoloko lifuna ukwazi ukuba uhleli nabani ngawo onke } \\
\text { amaxesha? }\end{array}$ & $\begin{array}{l}\text { Ewe } \\
(1)\end{array}$ & Hayi \\
\hline $\begin{array}{l}\text { 4. likubiza ngento yonke okanye likuthob' isidima phambi } \\
\text { kwabanye? }\end{array}$ & $\begin{array}{l}\text { Ewe } \\
(1)\end{array}$ & Hayi \\
\hline 5. likwenza uzive ungento yanto? & $\begin{array}{l}\text { Ewe } \\
(1)\end{array}$ & Hayi \\
\hline 6. liyakungxolisa okanye likuthuke? & $\begin{array}{l}\text { Ewe } \\
(1)\end{array}$ & Hayi \\
\hline 7. Likwenzakalisile emzimbeni & $\begin{array}{l}\text { Ewe } \\
(1)\end{array}$ & Hayi \\
\hline Zizonke: & & \\
\hline
\end{tabular}

\section{CTS-2 (beka u-X kwibhloko nganye oyikhethileyo)}

Kunyaka ophelileyo iqabane lakho...

\begin{tabular}{|l|l|l|}
\hline 1. Lakubetha & $\begin{array}{l}\text { Ewe } \\
(1)\end{array}$ & Hayi \\
\hline 2. Lakubetha ngento & Ewe & Hayi \\
\hline
\end{tabular}




\begin{tabular}{|c|c|c|c|}
\hline & (1) & & \\
\hline 3. Lakukrwitsha & $\begin{array}{l}\text { Ewe } \\
\text { (1) }\end{array}$ & $\mathrm{Ha}$ & \\
\hline 4. Lakubetha kakhulu & $\begin{array}{l}\text { Ewe } \\
\text { (1) }\end{array}$ & $\mathrm{Ha}$ & \\
\hline 5. Lakugrogrisa ngesixhobo & $\begin{array}{l}\text { Ewe } \\
\text { (1) }\end{array}$ & $\mathrm{Ha}$ & \\
\hline 6. Lasebenzisa isixhobo kuwe & $\begin{array}{l}\text { Ewe } \\
(1)\end{array}$ & $\mathrm{Ha}$ & \\
\hline 7. Lakwenza wabelana ngesondo ngaphandle kwekhondom & $\begin{array}{l}\text { Ewe } \\
\text { (1) }\end{array}$ & $\mathrm{Ha}$ & \\
\hline 8. Lasebenzisa amandla ukukwenza wabelane ngesondo nalo & $\begin{array}{l}\text { Ewe } \\
\text { (1) }\end{array}$ & $\mathrm{Ha}$ & \\
\hline $\begin{array}{l}\text { 9. lasebenzisa amandla ukwabelana ngesondo ngendlela } \\
\text { ezohlukeneyo }\end{array}$ & $\begin{array}{l}\text { Ewe } \\
(1)\end{array}$ & $\mathrm{Ha}$ & \\
\hline \multirow{2}{*}{\multicolumn{4}{|c|}{$\begin{array}{l}\text { Zizonke "Yes" points: } \\
\text { To be IPV eligible: Must score } 2 \text { or higher on NVC or score } 1 \text { or higher in CTS- } \\
2 \\
\text { NVC: } \\
\text { IPV eligible If YT-2: } \\
\text { If yes, complete remainder of scales } \\
\text { For those not IPV eligible: “Thank you very much for answering these } \\
\text { questions. We will not be in contact with you any further. Before I go, do you } \\
\text { mind if I quickly just ask what your age and race are for research purposes? } \\
\text { Age } \\
\text { Thank you." Race__ }\end{array}$}} \\
\hline & & & \\
\hline \multicolumn{4}{|c|}{ 4. VVS (beka u-X kwibhloko nganye oyikhethileyo) } \\
\hline $\begin{array}{l}\text { Le nto elandelayo yenzeka rhoqo kangakanani: } \\
\text { 0=Phantse ayizange okanye zange } \\
\text { 1=Rhoqo }\end{array}$ & wexesh & lide & \\
\hline $\begin{array}{l}\text { 1. Iqabane lakho libonakala liwabona amazwi akho okanye izenzo } \\
\text { zakho kakubi kunokuba wenza ubucinga. }\end{array}$ & 0 & 0 & 1 \\
\hline $\begin{array}{l}\text { 2. Xa ninengxaki enifanele niyisombulule, iba ngathi nidlalela } \\
\text { amaqela achaseneyo. }\end{array}$ & 0 & 0 & 1 \\
\hline 3. Uziva ulilolo kolu thando. & 0 & 0 & 1 \\
\hline $\begin{array}{l}\text { 4. Xa nixambulisana, omnye wenu uyarhoxa ... into ethetha } \\
\text { ukuthi, akafuni ukuthetha ngayo kwakhona okanye uvela ahambe. }\end{array}$ & 0 & 0 & 1 \\
\hline 5. Uyaloyika iqabane lakho. & 0 & 0 & 1 \\
\hline $\begin{array}{l}\text { 6. Uziva ngathi iimbono zakho okanye iimvakalelo zakho } \\
\text { azibalulekanga kolu thando Iwenu. }\end{array}$ & 0 & 0 & 1 \\
\hline
\end{tabular}




\begin{tabular}{|c|c|c|}
\hline $\begin{array}{l}\text { 7. Xa nixambulisana, iqabane lakho lisoloko lifuna "ukuphumelela" } \\
\text { yaye elifuni ukumamela ukuba wena uthini. }\end{array}$ & 0 & 0 \\
\hline 8. Unoloyiko lokwalela iqabane lakho isondo. & 0 & 0 \\
\hline \multicolumn{3}{|l|}{ Zizonke “Rhogo" points: } \\
\hline \multicolumn{3}{|l|}{ 5. HIRS (beka u-X kwibhloko nganye oyikhethileyo) } \\
\hline \multicolumn{3}{|l|}{$\begin{array}{l}\text { Ingaba uyavumelana nala mazwi alandelayo? } \\
\text { Ngenxa yobume bakho bokuba ne-HIV+... }\end{array}$} \\
\hline 1. Uza kuyibeka apha kwiqabane lakho & $\begin{array}{l}\text { Ewe } \\
(1)\end{array}$ & Hayi \\
\hline 2. Iqabane lakho liza kukuxhasa & $\begin{array}{l}\text { Hayi } \\
\text { (1) }\end{array}$ & $\begin{array}{l}\text { Ewe } \\
(0)\end{array}$ \\
\hline 3. Uthando lwenu luza lubi nangakumbi & $\begin{array}{l}\text { Ewe } \\
(1)\end{array}$ & Hayi \\
\hline $\begin{array}{l}\text { 4. Ukufumana unyango kuza kuba yingozi ngenxa yeqabane } \\
\text { lakho }\end{array}$ & $\begin{array}{l}\text { Ewe } \\
(1)\end{array}$ & Hayi \\
\hline 5. Uza kuziva ungakhuselekanga kuthando lwenu & $\begin{array}{l}\text { Ewe } \\
\text { (1) }\end{array}$ & Hayi \\
\hline $\begin{array}{l}\text { 6. Abanye baza kukunceda ukuba uziva ungakhuselekanga } \\
\text { eluthandweni lwenu }\end{array}$ & $\begin{array}{l}\text { Hayi } \\
\text { (1) }\end{array}$ & $\begin{array}{l}\text { Ewe } \\
(0)\end{array}$ \\
\hline 7. Akukhuselekanga ukuxoxa ngokuhlola i-HIV neqabane lakho & $\begin{array}{l}\text { Ewe } \\
(1)\end{array}$ & Hayi \\
\hline $\begin{array}{l}\text { 8. Akukhuselekanga ukuxoxa ngobume bakho be-HIV neqabane } \\
\text { lakho }\end{array}$ & $\begin{array}{l}\text { Ewe } \\
(1)\end{array}$ & Hayi \\
\hline 9. Uziva ukhuselekile ukuya kumadinga ezonyango & $\begin{array}{l}\text { Hayi } \\
\text { (1) }\end{array}$ & $\begin{array}{l}\text { Ewe } \\
(0)\end{array}$ \\
\hline 10. Woyika ukuba iqabane lakho lingazama ukukubulala & $\begin{array}{l}\text { Ewe } \\
(3)\end{array}$ & Hayi \\
\hline \multicolumn{3}{|c|}{$\begin{array}{l}\text { Zizonke: } \_ \text {(remember question } 10 \text { is worth } 3 \text { points and } 2,6 \& 9 \text { are } \\
\text { reverse scoring) }\end{array}$} \\
\hline \multicolumn{3}{|c|}{ 6. DA (beka u-X kwibhloko nganye oyikhethileyo) } \\
\hline \multicolumn{3}{|l|}{ Kunyaka ophelileyo... } \\
\hline $\begin{array}{l}\text { 1. Ingaba ubundlobongela buye bathi chatha kubudlelwane } \\
\text { benu? }\end{array}$ & Ewe (1) & Hayi \\
\hline 2. Ingaba iqabane lakho linompu? & Ewe (2) & Hayi \\
\hline 3. Have you left or broken up with your partner? & Ewe (2) & Hayi \\
\hline 4. Ingaba iqabane lakho likugrogrisa ngokukubulala? & Ewe (2) & Hayi \\
\hline $\begin{array}{l}\text { 5. Ingaba iqabane lakho lisebenzisa iziyobisi ezingekho } \\
\text { mthethweni ezifana (umz., i-tik, i-crack/cocaine, lifunxa i-glu, } \\
\text { njl.) okanye lixuba intsangu notywala okanye nezinye iziyobisi? }\end{array}$ & Ewe (1) & Hayi \\
\hline
\end{tabular}




\begin{tabular}{|c|c|c|}
\hline or mix dagga with other drugs? & & \\
\hline $\begin{array}{l}\text { 6. Ingaba iqabane lakho likhoboka lotywala okanye liyindla- } \\
\text { manzi? }\end{array}$ & Ewe (1) & Hayi \\
\hline 7. Ingaba iqabane lakho lakha lagrogrisa ngokuzibulala? & Ewe (2) & Hayi \\
\hline $\begin{array}{l}\text { 8. Ingaba iqabane lakho liyakulandela okanye lisoloko likubeke } \\
\text { elisweni okanye likushiyele imiyalezo egrogrisayo? }\end{array}$ & $\begin{array}{l}\text { Ewe } \\
\text { (.5) half }\end{array}$ & Hayi \\
\hline 9. Ingaba iqabane lakho liyazitshabalalisa izinto zakho? & $\begin{array}{l}\text { Ewe } \\
\text { (.5) half }\end{array}$ & Hayi \\
\hline $\begin{array}{l}\text { 10. Ingaba wakha wagrogrisa ngokuzibulala okanye wazama } \\
\text { ukuzibulala? }\end{array}$ & Ewe (0) & Hayi \\
\hline 11. Do you have a child that is not by your current partner? & Ewe (1) & Hayi \\
\hline \multicolumn{3}{|c|}{$\begin{array}{l}\text { Zizonke points } \quad \text { (remembering that questions } 2,3,4 \text {, and } 7 \text { count } 2 \\
\text { points each): } \\
\text { Time of End of Pre-test }\end{array}$} \\
\hline
\end{tabular}

Thank you for answering those questions. Do you mind if I ask what your age and race are?

Uhlanga: Iminyaka:

For Standard of Care group, state:

Script: Thank you so much for participating. We will contact you again in one month."

For Experimental groups, tally their scores and begin Safety plan.

Totals:

NVC:

CTS-2:

VVS:

HIRS:

DA:

\section{ONLY THOSE IN EXPERIMENTAL GROUP RECEIVE THEIR SCORE!}

Total Score:

Meaning of Score

$\begin{array}{llll}1 & 0-5 & 6 & 26-30 \\ 2 & 6-10 & 7 & 31-35 \\ 3 & 11-15 & 8 & 36-40 \\ 4 & 16-20 & 9 & 41-45 \\ 5 & 21-25 & 10 & 46-50\end{array}$


Appendix L

\section{Initial Safety Plan isiXhosa}

\section{Initial Safety Plan IsiCwangciso senkQubo yoKhuseleko: (Umbhalo):}

Ngokweempendulo zakho kwimibuzo esixelela ngendlela okhuseleke ngayo noziva ngayo ngomntu wakho ufumene kwi-10 apho i-10 libonisa ukuba usengozini enkulu. Uziva njani ngokuva le nkcazelo? (2)

Ubume bakho be-HIV busebutsha yaye awukhange ufumane ixesha elaneleyo lokucinga ngayo. Kusenganzima ukucinga into eninzi ngokwangoku, kodwa kubalulekile ukucinga ngendlela oya kuhlala ukhuseleke ngayo kumntu wakho ekubeni unezi ziphumo.

Ndikuphathele inkcazelo ebalulekileyo. Abantu basetyhini bafumana ubundlobongela ngeendlela ezintathu eziphambili: ngokwasengqondweni, emzimbeni nangokwesondo. Zonke ezi ntlobo zokuxhatshazwa zinokuthi chatha emva kokufunyaniswa kwe-HIV. Imizekelo yokuxhatshazwa ngokwasengqondweni kukubizwa ngento yonke, ukuthob' isidima okanye ukuzama ukukulawula. Imizekelo yokuxhatshazwa emzimbeni kukubethwa, ukukhatywa, okanye ukukuqhwaba ngempama. Imizekelo yokuxhatshazwa kwezesondo kukukunyanzela wabelane ngesondo naye okanye wabelane ngesondo ngaphandle kwekhondom.

Abasetyhini abaxhatshazwayo okanye abanamaqabane anobundlobongela, baba semngciphekweni othe chatha wokufumana ubundlobongela obungakumbi emveni kokufunyaniswa bene-HIV. Abanye abantu basetyhini baba kwingozi enkulu xa bexelela amaqabane abo ngobume babo be-HIV okanye xa bezama ukufumana unyango lwezamayeza okanye unyango lwe-HIV. Amaqabane axhaphazayo anokuzama ukubathintela ekufumaneni unyango okanye xa besiya kuthatha amayeza.

Nangona isenokuba lixesha eliyingozi kwezothando lwakho, abasetyhini abaninzi bakhetha ukuwaxelela amaqabane abo ngobume babo ukuze nawo ahlolwe okanye afumane unyango. Ukuba ugqiba ekubeni ulixelele iqabane lakho, kubalulekile ukuvela nesicwangciso seyona ndlela ikhuselekileyo ongakwenza ngayo oko. Sisigqibo sakho ukuba ugqiba ekubeni ulixelele iqabane lakho ngobume bakho be-HIV.

Ingaba kulungile xa sisithi gqaba-gqaba nje ngezinye zeendlela onokuhlala ukhuselekile ngazo kumntu wakho?

13. Kukho abantu abaninzi ebomini bakho naseluntwini abanokukunceda ukuba uziva usengozini emntwini wakho. Baquka ugqirha wakho, umongikazi, umcebisi, umfundisi okanye umthandazeli okanye inkonzo ejongene nabantu abaxhatshazwayo.

$>$ Kukho iminxeba exakekileyo esimahla neyimfihlo onokuyitsalela xa udinga umntu onokuthetha naye okanye indawo kaxakeka. Enye yile nombolo: 0800-150-150. Kubalulekile ukuba uyibhale kwindawo 
ekhuselekileyo ukuze iqabane lakho lingayiboni. Zeziphi ezinye zeendawo onokuyibeka kuzo? (umz.: emsebenzini okanye emnxebeni wakho qha uyinike igama elahlukileyo). (3)

14. Abanye abantu bakhetha ukuthetha okanye ukufuna uncedo kubantu ababaziyo. Oku kusenokuquka ukuthetha nosapho lwakho okanye abahlobo okanye umntu wasekuhlaleni. Ukuthetha nelungu losapho okanye umhlobo kungakunceda xa ngexesha likaxakeka.

$>$ Cinga ukuba leliphi kumalungu osapho okanye kubahlobo ekukhuselekileyo ukuthetha naye (ongasayi kulixelela iqabane lakho). Ungambiza lo mntu ngexesha likaxakeka (unokusebenzisa igama elithile elaziwa nini nobabini xa uchaza ukuba ufuna uncedo).

15. Kukwakho noncedo olusemthethweni xa iqabane linobundlobongela. Eminye imizekelo kukubiza amapolisa, ukunxibelelana negqwetha okanye ukufaka umyalelo wokhuseleko.

> Ngokusiseko, umyalelo wokhuseleko uya kulithintela iqabane lakho ekungeneni kwikhaya lakho okanye emsebenzini wakho, okanye ekunxibelelaneni nabantwana bakho. Kwakhona, le miyalelo inceda ekuthinteleni izenzo ezingakumbi zokuxhaphaza. Ingaba ungathanda ukufumana inombolo ongafumana kuyo inkcazelo malunga nomyalelo wokhuseleko? (ifanele igcwaliswe njengesicelo okanye ingxelo efungelweyo kwisebe lamapolisa asekuhlaleni) okanye kwi-ofisi elikomkhulu (ePitoli) apha +27 (0) 123931000.

> Yigcine kwindawo ekhuselekileyo (kwindlu yelungu losapho, kwikhaya lakho okanye kwipesi yakho kwindawo efihlakeleyo njengakwityhubhu ye-lipstick okanye ubhale igama lenkonzo eyahlukileyo kuyo (isalon yeenwele, indawo ethengisa ukutya, njl)

16. Abanye abantu bazama ukwenza izinto ngenjongo yokuphepha ukuxhatshazwa ngamaxesha ayingozi. Xa kuvuka umlo, abanye abantu bazama ukuwathomalalisa umsindo amaqabane abo, kodwa khumbula ukuba indlela eliziphatha ngayo asilotyala lakho okanye uxanduva lwakho.

$>$ Ingaba akho amaxesha athile ocinga ukuba kungakuhle ukuba uhlalele kude engozini? (4)

17. Abanye abantu bazama ukuxhathisa ukuxhatshazwa xa kuvela. Imizekelo yoku kukuzilwela, ukufihla umpu kwindawo eyaziwa nguwe wedwa, ukuzama ukuhamba okanye ukuphelisa into ebenihlangene ngayo, okanye ukubaleka okanye ukuzimela xa kuvela ingxaki. Uphando lubonise ukuba ukuxhathisa ukuxhatshazwa kunokuba yingozi enkulu yaye kungabenza buthi chatha ubundlobongela.

$>$ Ingaba ucinga ngokumshiya umntu wakho? (5) Ewe Hayi Ukuba kunjalo, Kuhle ukwenza isicwangciso kuba eli lixesha eliyingozi kakhulu kwezothando ezinobundlobongela. (6) Isicwangciso sesi

18. Ezinye izicwangciso ziquka ukulungiselela imini kaxakeka nokufihla imali, iinombolo ezibalulekileyo nezinto zobuqu xa kunokufuneka umke buphuthuphuthu. Kukwakuhle ukuceba kwangaphambili ukuba uza kuya phi 
xa kuvuka umlo endlwini yakho (uye kwigumbi elikhuselekileyo apho kungekho zixhobo okanye izinto ezibukhali)

Ekuqukumbeleni lo mnxeba, ndingathanda ukukunceda wenze isicwangciso ngendlela onokuzikhathalela ngayo ngokukhuselekileyo, ekubeni kukho ezi ziphumo zintsha kunye nengozi yeqabane lakho.

Ukuba ugqiba ekubeni uxelele iqabane lakho ngobume bakho, ngoku okanye ngelinye ixesha, zeziphi ezinye zeendlela onokuzikhusela ngazo? (7)

Ukuba ukrokrela ukuba iqabane lakho lilala nomnye umntu, kubalulekileyo ukwazi ukuba ukuxambulisana neqabane lakho ngaloo nto kungabangela ubundlobongela obungakumbi. Ukusebenzisa iikhondom, emveni kokufumana iziphumo ze-HIV kuye kwabonisa ukuba kuyanceda ekosuleleni abanye abantu kodwa ukucela iqabane lakho lisebenzise iikhondom kuye kwabonisa ukuba kuye kwakho ubundlobongela obuthe chatha. Ukuba uza kuqalisa ukusebenzisa iikhondom, cinga ngeyona ndlela ekhuselekileyo yokuthetha neqabane lakho ngaloo nto.

Kuhle kakhulu ukuba emveni kweziphumo uye ngoko nangoko ekliniki ukuya kufumana unyango lwezamayeza. Ude ube ugqiba ekubeni ulixelele iqabane lakho ngeziphumo, ungazifumana njani ngokukhuselekileyo iinkonzo zamayeza? Ingaba kufuneka wenze icebo lokuxelela iqabane lakho ukuba uya kwenye indawo? Uza kuthini? (8)

Ukuba umlinganiselo we-CD4 uphansi (ungaphantsi kwama-500), kuya kucetyiswa ukuba uqalise unyango lwee-antiretroviral. Icebo elixhaphakileyo kukuwafihla la machiza kwiqabane elixhaphazayo. Zeziphi ezinye iindlela ongakwenza ngazo oku ukuba kuyimfuneko? (ngokomzekelo, ukufaka iipilisi kwibhotile ye-Tylenol kunye/okanye nokuzigcina kuwe). Uza kwenza njani wena? (9)

Ingaba ikho enye into esingakunceda ngayo namhlanje ukuze ufumane unyango oludingayo lwezamayeza ngokukhuselekileyo ukuze uzikhathalele ngokwasemzimbeni nangokwasengqondweni? (10)

\section{Time safety plan ends}

Qualitative answers from Safety Plan:

1. Level of danger is:

2. Feelings after hearing level of danger:

3. If accepted free hotline number, where is safe place to keep number:

4. Times/situations need to stay away from partner: 
5. Yes or No (to leaving relationship)

6. Plan for leaving is:

7. Ways to protect self if discloses to partner:

8. Plan to safely get to HIV medical care or clinic:

9. Plan to keep HIV medication safe (if applicable):

10. Anything else we can help with to keep physically and emotionally safe: 
Post-test Experimental isiXhosa

\begin{tabular}{|c|c|c|c|}
\hline \multicolumn{4}{|l|}{ Uvavanyo lwaMva } \\
\hline \multicolumn{4}{|l|}{ 1. HIRS } \\
\hline $\begin{array}{l}\text { Ukususela ekufunyanisweni kwakho ukuba une-HIV... } \\
\text { nala mazwi alandelayo? }\end{array}$ & \multicolumn{3}{|c|}{ Ingaba uyavumelana } \\
\hline 1. Usenkathazweni kwiqabane lakho & \multicolumn{2}{|c|}{ Ewe } & $\begin{array}{l}\mathrm{Ha} \\
\text { yi }\end{array}$ \\
\hline 2. Iqabane lakho liyakuxhasa & \multicolumn{2}{|c|}{ Ewe } & $\begin{array}{l}\mathrm{Ha} \\
\mathrm{yi}\end{array}$ \\
\hline 3. Uthando lwenu lusengxakini enkulu & \multicolumn{2}{|c|}{ Ewe } & $\begin{array}{l}\mathrm{Ha} \\
\mathrm{yi}\end{array}$ \\
\hline 4. Ukufumana unyango bekuyingozi ngenxa yeqabane lakho & \multicolumn{2}{|c|}{ Ewe } & $\begin{array}{l}\mathrm{Ha} \\
\mathrm{yi}\end{array}$ \\
\hline 5. Uziva ungakhuselekanga kolu thando lwenu & \multicolumn{2}{|c|}{ Ewe } & $\begin{array}{l}\mathrm{Ha} \\
\mathrm{yi}\end{array}$ \\
\hline $\begin{array}{l}\text { 6. Abanye bebekhona ukuze bakuncede xa uziva } \\
\text { ungakhuselekanga kumntu wakho }\end{array}$ & \multicolumn{2}{|c|}{ Ewe } & $\begin{array}{l}\mathrm{Ha} \\
\mathrm{yi}\end{array}$ \\
\hline $\begin{array}{l}\text { 7. Bekungakhuselekanga ukuxoxa ngokuhlola i-HIV neqabane } \\
\text { lakho }\end{array}$ & \multicolumn{2}{|c|}{ Ewe } & $\begin{array}{l}\mathrm{Ha} \\
\mathrm{yi}\end{array}$ \\
\hline $\begin{array}{l}\text { 8. Bekungakhuselekanga ukuxoxa ngobume bakho be-HIV } \\
\text { neqabane lakho }\end{array}$ & \multicolumn{2}{|c|}{ Ewe } & $\begin{array}{l}\mathrm{Ha} \\
\mathrm{yi}\end{array}$ \\
\hline 9. Ubuziva ukhuselekile ukuya kumadinga ezonyango & \multicolumn{2}{|c|}{ Ewe } & $\begin{array}{l}\mathrm{Ha} \\
\mathrm{yi}\end{array}$ \\
\hline $\begin{array}{l}\text { 10. Ubunoloyiko lokuba iqabane lakho liza kuzama } \\
\text { ukukubulala }\end{array}$ & \multicolumn{2}{|c|}{ Ewe } & $\begin{array}{l}\mathrm{Ha} \\
\mathrm{yi}\end{array}$ \\
\hline \multicolumn{4}{|l|}{ 2.SS } \\
\hline \multicolumn{4}{|l|}{ Ingaba uyavumelana nezi zinto zilandelayo: } \\
\hline \multicolumn{2}{|l|}{ 1. Iyonke inkcazelo othe wayifumana ngezothando ibe luncedo } & Ewe & Hayi \\
\hline \multicolumn{2}{|l|}{ 2. Bekuluncedo ukubuzwa ngeenzingo kwezothando } & Ewe & Hayi \\
\hline \multicolumn{2}{|l|}{$\begin{array}{l}\text { 3. Unqwenela ukuba akwaba ubunokuxoxa ngakumbi ngeenzingo } \\
\text { kwezothando }\end{array}$} & Ewe & Hayi \\
\hline \multicolumn{2}{|l|}{ 4. Ubungakhululekanga ukuxoxa ngeenzingo zothando } & Ewe & Hayi \\
\hline \multicolumn{2}{|l|}{ 5. Uyazisola ngokuthetha ngeenzingo zothando lwakho } & Ewe & Hayi \\
\hline \multicolumn{2}{|l|}{$\begin{array}{l}\text { 6. Ukuthetha ngeenzingo zothando lwam kundibeke kwenkulu } \\
\text { ingozi }\end{array}$} & Ewe & Hayi \\
\hline \multicolumn{2}{|l|}{ 7. Linkonzo ozifumeneyo zikubeke kwenkulu ingozi } & Ewe & Hayi \\
\hline \multicolumn{4}{|l|}{ 3.FAS } \\
\hline 1. Ingaba usisebenzisile isicwangciso & & Hayi & \\
\hline
\end{tabular}




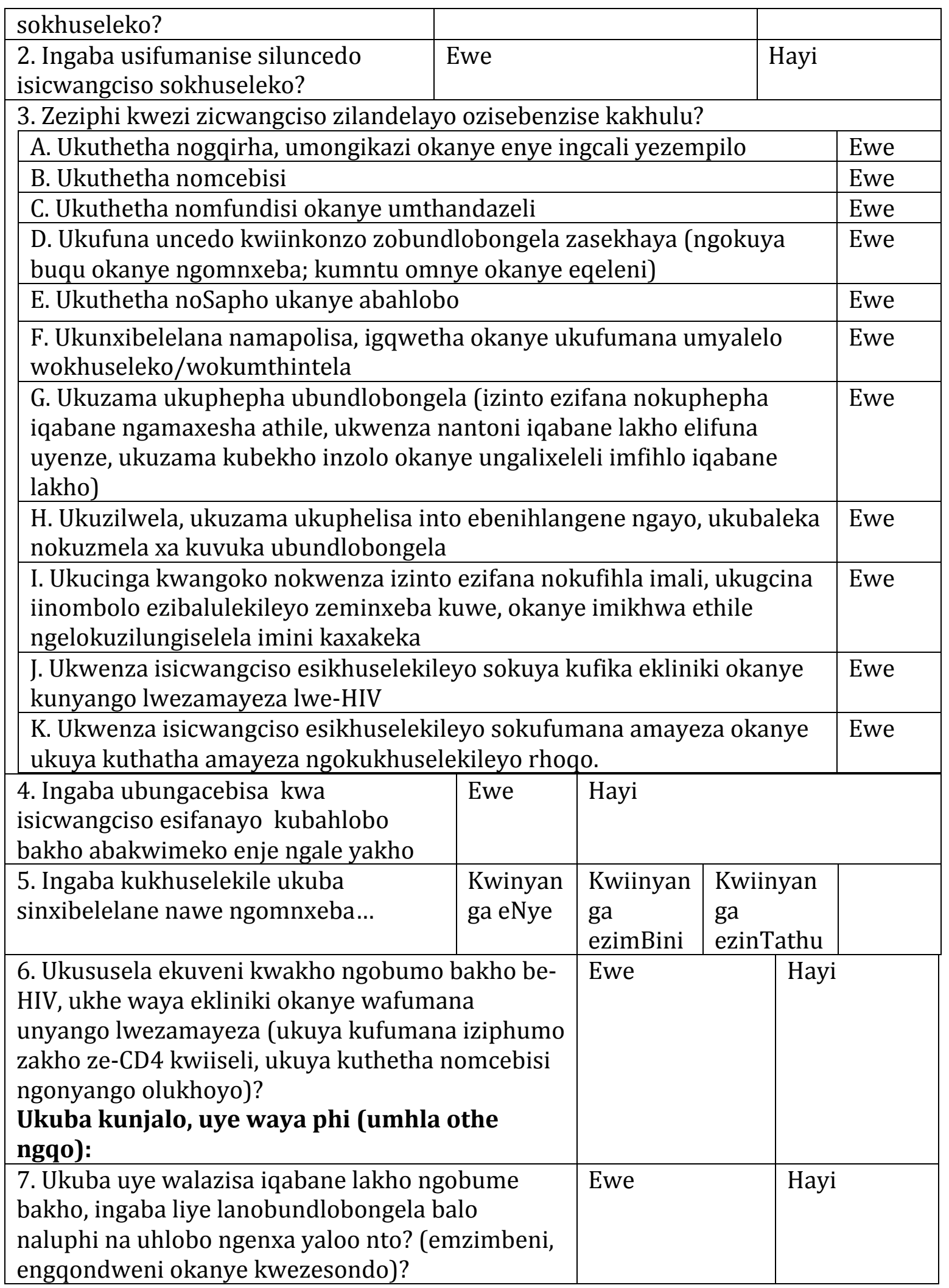


Post-test Standard of Care isiXhosa

\begin{tabular}{|c|c|c|}
\hline \multicolumn{3}{|c|}{ Uvavanyo lwaMva Date ___ Time call begins } \\
\hline \multicolumn{3}{|l|}{$\begin{array}{ll}1 . \text { HIRS } \\
\end{array}$} \\
\hline \multicolumn{3}{|l|}{$\begin{array}{l}\text { Ukususela ekufunyanisweni kwakho ukuba une-HIV... } \\
\text { uyavumelana nala mazwi alandelayo? }\end{array}$} \\
\hline 1. Usenkathazweni kwiqabane lakho & Ewe & $\begin{array}{l}\mathrm{Ha} \\
\mathrm{yi}\end{array}$ \\
\hline 2. Iqabane lakho liyakuxhasa & Ewe & $\begin{array}{l}\mathrm{Ha} \\
\mathrm{yi}\end{array}$ \\
\hline 3. Uthando lwenu lusengxakini enkulu & Ewe & $\begin{array}{l}\mathrm{Ha} \\
\mathrm{yi}\end{array}$ \\
\hline 4. Ukufumana unyango bekuyingozi ngenxa yeqabane lakho & Ewe & $\begin{array}{l}\mathrm{Ha} \\
\mathrm{yi}\end{array}$ \\
\hline 5. Uziva ungakhuselekanga kolu thando lwenu & Ewe & $\begin{array}{l}\mathrm{Ha} \\
\text { yi }\end{array}$ \\
\hline $\begin{array}{l}\text { 6. Abanye bebekhona ukuze bakuncede xa uziva ungakhuselekanga } \\
\text { kumntu wakho }\end{array}$ & Ewe & $\begin{array}{l}\mathrm{Ha} \\
\mathrm{yi}\end{array}$ \\
\hline $\begin{array}{l}\text { 7. Bekungakhuselekanga ukuxoxa ngokuhlola i-HIV neqabane } \\
\text { lakho }\end{array}$ & Ewe & $\begin{array}{l}\mathrm{Ha} \\
\text { yi }\end{array}$ \\
\hline $\begin{array}{l}\text { 8. Bekungakhuselekanga ukuxoxa ngobume bakho be-HIV } \\
\text { neqabane lakho }\end{array}$ & Ewe & $\begin{array}{l}\mathrm{Ha} \\
\mathrm{yi}\end{array}$ \\
\hline 9. Ubuziva ukhuselekile ukuya kumadinga ezonyango & Ewe & $\begin{array}{l}\mathrm{Ha} \\
\mathrm{yi}\end{array}$ \\
\hline 10. Ubunoloyiko lokuba iqabane lakho liza kuzama ukukubulala & Ewe & $\begin{array}{l}\mathrm{Ha} \\
\mathrm{yi}\end{array}$ \\
\hline \multicolumn{3}{|l|}{ 2.SS } \\
\hline \multicolumn{3}{|l|}{ Ingaba uyavumelana nezi zinto zilandelayo: } \\
\hline 1. Iyonke inkcazelo othe wayifumana ngezothando ibe luncedo & $\begin{array}{l}\text { Ew } \\
\text { e }\end{array}$ & Hayi \\
\hline 2. Bekuluncedo ukubuzwa ngeenzingo kwezothando & $\begin{array}{l}\text { Ew } \\
\text { e }\end{array}$ & Hayi \\
\hline $\begin{array}{l}\text { 3. Unqwenela ukuba akwaba ubunokuxoxa ngakumbi ngeenzingo } \\
\text { kwezothando }\end{array}$ & $\begin{array}{l}\text { Ew } \\
\text { e }\end{array}$ & Hayi \\
\hline 4. Ubungakhululekanga ukuxoxa ngeenzingo zothando & $\begin{array}{l}\text { Ew } \\
\text { e }\end{array}$ & Hayi \\
\hline 5. Uyazisola ngokuthetha ngeenzingo zothando lwakho & $\begin{array}{l}\text { Ew } \\
\text { e }\end{array}$ & Hayi \\
\hline 6. Ukuthetha ngeenzingo zothando lwam kundibeke kwenkulu & Ew & Hayi \\
\hline
\end{tabular}




\begin{tabular}{|c|c|c|c|c|}
\hline \multicolumn{3}{|l|}{ ingozi } & e & \\
\hline \multicolumn{3}{|c|}{ 7. Linkonzo ozifumeneyo zikubeke kwenkulu ingozi } & Ew & Hayi \\
\hline $\begin{array}{l}\text { 8. Ingaba kukhuselekile ukuba } \\
\text { sinxibelelane nawe ngomnxeba... }\end{array}$ & Kwinyanga eNye & $\begin{array}{l}\text { Kwiinya } \\
\text { nga } \\
\text { ezimBin } \\
\text { i }\end{array}$ & \multicolumn{2}{|c|}{$\begin{array}{l}\text { Kwiinyanga } \\
\text { ezinTathu }\end{array}$} \\
\hline \multicolumn{2}{|c|}{$\begin{array}{l}\text { 9. Ukususela ekuveni kwakho ngobumo bakho be-HIV, } \\
\text { ukhe waya ekliniki okanye wafumana unyango } \\
\text { lwezamayeza (ukuya kufumana iziphumo zakho ze-CD4 } \\
\text { kwiiseli, ukuya kuthetha nomcebisi ngonyango } \\
\text { olukhoyo)? } \\
\text { Ukuba kunjalo, uye waya phi (umhla othe ngqo): }\end{array}$} & Ewe & \multicolumn{2}{|c|}{ Hayi } \\
\hline \multicolumn{2}{|c|}{$\begin{array}{l}\text { 10. Ukuba uye walazisa iqabane lakho ngobume bakho, } \\
\text { ingaba liye lanobundlobongela balo naluphi na uhlobo } \\
\text { ngenxa yaloo nto? (emzimbeni, engqondweni okanye } \\
\text { kwezesondo)? }\end{array}$} & Ewe & \multicolumn{2}{|c|}{ Hayi } \\
\hline \multicolumn{2}{|c|}{$\begin{array}{l}\text { 11. Kwinyanga ephelileyo, ingaba ukhe wafumana } \\
\text { uncedo ngenxa yokuxhatshazwa kwezothando kuyo } \\
\text { nayiphi na yale mithombo elandelayo: emapoliseni, } \\
\text { umnxeba oxakekileyo okanye inkqubo yobundlobongela } \\
\text { basekhaya, umcebisi, umfundisi/okanye umthandazeli } \\
\text { okanye naphi na? }\end{array}$} & Ewe & \multicolumn{2}{|c|}{ Hayi } \\
\hline
\end{tabular}


Appendix 0

\section{Informed Consent isiZulu}

\section{Subject Informed Consent Document \\ HIV Screening and Intimate Partner Violence Safety Study}

Sponsor(s) name \& address:University of Louisville, Louisville, KY and Shout-itNow, Cape Town, SA

Investigator(s) name \& address: Primary Investigator Michiel A. van Zyl and Coinvestigator Leslie Lauren Brown: 2217 South Third St. Louisville, KY 40292, USA.

Site(s) where study is to be conducted: Gauteng province and telephone calls will be made from Cape Town, South Africa.

Phone number for subjects to call for questions:021 7134414

\section{Introduction and Background Information}

Umenyiwe uzoxhasa uchwaningo. Ucwaningo luholwa uDr. Riaan van Zyl, PhD;Lauren Brown, LCSW, PhD Candidate and Shout-it-Now. Lolucwaningo luxhaswe yiuniese yase Louisville, nomyaga we Kent School of Social Work. Lolucwaingo lozothathela kuwownke ahambayo umeluleki wenculaza nokuhlolwa emalokishini aseGoli we S-N loluhambo luzokwenzala esikhathi esihleliwe. Ukuthintana kwalocwaningo kuzovela ngocingo, ngaphansi kwe Shout-it-Now, elitholakala eTokai, eMpuma koloni. Abantu abalinganiswe ku 225 bazoba inxenye yalokhu.

\section{Isizathu}

Isizathu salolucwaningo ukubona ukuthi abantu besimame baunani uma bazi ngobudlelwane nokuphepha mabehlolwaminculaza noma ukuba necebo uma esehloliwe.

\section{Izindlela}

Kolocwaningo, uzothintwa ngocingo ngakusasa emva kokuthola usizo nababe Shout-it-Now.

Ukuxhasana kungavela ngedlela ezimbili.

Uma uvela kwisombe sokuqaba, ukuhlolwa kwakho ngocingo kuzo bakana nokuxhasa ucwaningo kabanzi. Sizokuthinta ukuze sithole umbono wakho 
ngololucwaningo nemibuzo. Sifuna ukwazi ukuthi ucabanga ukhuthi lemibuzo inosizo. Eminye imibuzo yasesombeni sesibili ingabuzwa esomboni sokuqala. Lama intavu nyeke enkodiwe kodwa kozo bhalwa phansi nase komputheni. Uma ungenxanye yesombe sesibili kwalolucwaningo, uzobuzwa imibuzo ezokusiza ukuthi sibone ukuthi ubudlelwane bakho buphephile yini. Eminye imibuzo ikhandelwe lolucwaningo. Eminye imibuzo ejwayelekile ukubuzwa umhlaba wonke jikele. Eminye imibuzo ibheke kwabantu abazwanayo ngokwenganekwane ngokozimba nangokwecansi udlame nokunye okomoyo ukuphepha. Ikhona eminye imibuzo mayelana nokwazi kwakho okwayenzeka kudala nomlingani wakho onodlame nemizwa yakho mayelana nodlame ebudlelwaneni. Ungathinwa 2-3 isikhathi mayelana locwaningo okokuqala ubuzwe imibuzo kanti nasekugcineni. Imibuzo yokuqala ingathatha imizuzu eqala ku 8 kuya ewu 20 ukuze uphendule. Kunemibuzo engu 46 emifishane uma besaqala ukukuthinta kanti egugcineni iba ngu 18-30 emifishane. Uma eminye imibuzo ingakuphathi khahle, ungakhetha ukungayiphenduli. Uzoqhubeka uthole usizo luka S-N noma ungaphendulanga yonke imibuzo. Amalungu esombeni sesibili azoxutshwa befakwe emabandleni ukuze bengatholi usizo olufanayo.

\section{Potential Risks}

Lolucwaningo alunalo ubugozi obutize, kodwa ubungozi kuvela uma kuhlolwa inculaza, nokuhlolwa kwabantu abanaba lingani abanodlame. Ubungozi obuvela uma kuhlolwa ukuthuka kokuthi umuntu okwaziyo ekubone, lokukungenyuka uma usuthola ukuthi unesifo senculaza. Uma umuntu esethola ukuthi uneculaza uzizwa ngathi uyagxekwa umdeni wakhe nomphakathi. Ungagena engozini kakhulu uma ele ebudlelaleni wakho kunodlame vele. Ubungozi kahle kahle kololucwaningo ukuzizwa ungaphathekile kahle uma uphendula imibuzo enzima engawe noma ezinocansi kakhulu, ukuphendula lemibuzo kungenza ucabange izino ezenzeka kudala ezibuhlungu. Uma lokhu kwenzeka uzonikezwa imimimigwane yomuntu ongakusiza oseduze. Obunye ubungozi ukuthi I Shout-it Now izosebenzisa ucingo ukuxhumana nawe. Lokhu kungaba ngozi uma umlingani wakhe ethola. Ungayiphenduli ucingo uma kungaphephile. Kubonakala ngathi imiphumela emihle mining ukundlula emibi.

\section{Imiphumela Emihle}

Lolucwaningo linemipumela emihle eminingi. Ungazizwa uxhaswa kancono nezinto zenzeka kalula. Uzothola ukuthi lemibuzo ikwenze ucabanga kancono ngobudlelwane bako futhi ikwenze ufune usizo emundeni nasemphakathini ukuze uzizwe uphephile. Kukho konke locwaningo linethemba lokuthuthu abantu bathole usizo futhi bazizwe bephephile ucwaningo lukhombisa ukuthi abantu besifazane ababeyinxhenye yaloluhlelo bathole usizo. Futhi ulwazi lulana kulolucwaningo kuzokwenza abanye abantu besimame bethole usizo nedlela zokutho imithi yenculaza. Ngokutho imithi yabo abantu besimame bazoziwa bephephile, bezizwe becqusekile futhi begomqemane bephila impilo ezinde. 
Lemiphumela enganza kwehle ukuthelelana kwenculaza nabantu bengasafi bebulawa inculaza. Noma ulwazi kulocwaningo kungathinti wena bukhoma lizosiza abanye.

\section{Isinxephezelo}

Uma uwungenela lolucwaningo, uhlale lizo liyophela, igama lakho lizongena kumncitiswano uzothola i-pod yamahala. Futhi uzotho ukuhlolwa kwamahala kwi HIV/TB kanye umeluleki wamahala. Uma ungafuni ukuba yinxenye yocwaningo uzothola ukuhlolwa kwamahala kodwa nyeke umthole umeluleki wamahala. .

\section{Yimfihlo}

Imfihlo egcelwe nyeke uyethembise. Imfihlo zakho zizo ikelwa umthetho. Uma imiphumela yalolucwaningo iezwa igama lakho nyeke ivezwe, kodwa laba bazokwaz: The University of Louisville Institutional Review Board, Human Subjects Protection Program Office, and Privacy Office.

Office for Human Research Protections (OHRP)

Icala necala lizothola inobolo yayo ukuze igama lakho alaziwa emuninigwano yakho. Umninigwano igcineka kahle iqhembu lase Ningizimu Afika. Uma umninigwane isiqediwe lonke ulwazi selizovela kodwa hayi amagamo inombolo zamacala, bese zithunyelwa komseshi oqavile. Ukungena kuwu lamakomputha akukho lula noba ku khona igama lemfihlo elivalile nekomputha ine-HIPPA (follow privacy guidelines of the Health Insurance Portability and Accountability Act). Eminye imininingwano izogcineka kwi CD bese ivalelwa kukabin kwamaseshi oqaile emahoisini wakhe.

\section{Ozenzelayo}

Ukungenela kulento uyazinkela. Ungakhetha ukunga ngeneli. Uma ungenile ungakhetha ukushiya phansi noma yinini, nyeke uphuthelwe yilutho.

\section{Research Subject's Rights, Questions, Concerns, and Complaints}

Uma unenkinga noma ikhalazo ngocwaningo ngabasebenzi, unezinto eziwu 3 ongazenza.Ungaxhumana noNhlokomseshi ku 502-852-2430 (Emelika).

Uma unemibuzo mayelana namalungelo wakho ngocwaningo, imibuzo, ikhalazo noma inking, ungashayela Human Subjects Protection Program Office (HSPPO) (502) 852-5188.

Ungaxoxisana uma unemibuzo ngama lungelo wakho ngemfihlo, nelungu le Institutional Review Board (IRB) noma HSPPO isebenzi sakhona. I IRB ilungu elizimele lecomidi elihlanganiswe amalungu eyuniesi yomphakathi, amalungu 
noma isebenzi zenhlagano, kanye namalungu omphakathi abangahlangene nalenhlangano I-IRB iyawabukisisa lolucwaniningo.

Uma ufuna ukukhuluma nomuntu ngaphandle kwenivesi ungathinta 1-877-8521167. Uzonikezwa ithuba lokukhuluma nanoma umuphi umbuzo, ikhalazo noma inking ngemfihlo. Lolucingo lusebenza amahora angu 24, kodwa labantu abasebenzi euniesi yase Louisville.

Leliphepha lizokutshela ukuthi kuzokwezalani uma ukhetha ukuba inxenye yalolucwaningo. Ukusiyina kwakho kuchaza ukuthi yonkinto ikhulunyiwe nawe, yonke imibuzo iphenduliwe futhi uzoba yinxenye yocwaningo. Leliphepha akusikona ikotlaki Amalungelo wakho awayi ndawo uyawagcine ngokusiyina leliphepha. Uzonikezwa alinye iphepha ukuze nawe ubenerekordi.

Igama elibhaliwe isikhonzi

\section{Language preference:}

isiZulu Xhosa

Sesotho

English

Inombolo yocingo yesikhozi:

Isayini yesikhozi/Omele Obomthetho

Usuku Lokusayina

Isayini yomuntu ochazayo leliformi (uma ngaphandle komhloli)

Usuku Lokusayina

Isayini yoseshi

Usuku Lokusayina

AMAGAMA WABASESHI

INOMBOLO

Michiel A. van Zyl, PhD

$1-502-852-2430$

Lauren Brown, LCSW, PhD Candidate 1-502-852-2430 
Pre-test: Danger Indicator isiZulu

\section{Pre-test Zulu SOC \& Exp Groups}

Script: "Hi, this is with Shout-it-Now. I was given your information from my shout-it-now teammate because you agreed to participate in a brief study with us. This will take about 20 minutes or more. Are you still willing to participate in this study? Great. Is this a safe time to talk? Okay, I am going to ask you some questions now."

\begin{tabular}{|l|l|l|}
\hline \multicolumn{3}{|c|}{ Pre-test 1 RS } \\
\hline \multicolumn{2}{|c|}{} \\
\hline $\begin{array}{l}|c| \\
\text { 1. Unomuntu ozwana naye njengamanje? }\end{array}$ & Yebo & Cha \\
\hline $\begin{array}{l}\text { 2. (Uma uyebo ku 1, ndula u2): Uzithola uthandana esikhathini } \\
\text { esiningi }\end{array}$ & Yebo & Cha \\
\hline No Total for RS (does not count towards total) but is used for eligibility) \\
\hline \multicolumn{2}{|c|}{ 2. Put an X in each chosen block) } \\
\hline $\begin{array}{l}\text { Ungathi umlingani wakho manje uya... } \\
\text { 1. Uzama ukunciphisa ubudlelwane bakho nabangani }\end{array}$ & $\begin{array}{l}\text { Yebo } \\
\text { nomndeni wakho? }\end{array}$ & Cha \\
\hline $\begin{array}{l}\text { 2. Unesikhwele noma unesiphano? } \\
\text { Yebo } \\
\text { 3. Ufuna ukwazi ukuthi ukuphi ngaso sonke isikhathi? }\end{array}$ & $\begin{array}{l}\text { Cha } \\
(1)\end{array}$ & Cha \\
\hline $\begin{array}{l}\text { 4. Ukubiza amagama noma ukuwisa umoya uma ninabanye } \\
\text { abanthu? }\end{array}$ & $\begin{array}{l}\text { Yebo } \\
(1)\end{array}$ & Cha \\
\hline 5. Ukwenza uzizwe ngathi awulutho noma awunamusebenzi? & $\begin{array}{l}\text { Yebo } \\
(1)\end{array}$ & Cha \\
\hline
\end{tabular}




\begin{tabular}{|c|c|c|}
\hline 6. Uyakwethuka noma akuthethise? & $\begin{array}{l}\text { Yebo } \\
\text { (1) }\end{array}$ & Cha \\
\hline 7. Ulimaze umzimba wakho & $\begin{array}{l}\text { Yebo } \\
\text { (1) }\end{array}$ & Cha \\
\hline \multicolumn{3}{|c|}{ Total “Yebo" points: } \\
\hline \multicolumn{3}{|c|}{ 3. CTS-2 (Put an X in each chosen block) } \\
\hline \multicolumn{3}{|l|}{ Onyakeni ondlule umlingani wakho... } \\
\hline 1. Ukushayile & $\begin{array}{l}\text { Yebo } \\
\text { (1) }\end{array}$ & Cha \\
\hline 2. Ukushaye ngokutize & $\begin{array}{l}\text { Yebo } \\
\text { (1) }\end{array}$ & Cha \\
\hline 3. Ukuklinyile & $\begin{array}{l}\text { Yebo } \\
\text { (1) }\end{array}$ & Cha \\
\hline 4. Uku shayile kakhulu & $\begin{array}{l}\text { Yebo } \\
(1)\end{array}$ & Cha \\
\hline 5. Songela ngesikhali & $\begin{array}{l}\text { Yebo } \\
(1)\end{array}$ & Cha \\
\hline 6. Wasebenzisa isikhali kuwe & $\begin{array}{l}\text { Yebo } \\
(1)\end{array}$ & Cha \\
\hline $\begin{array}{l}\text { 7. Wakwenza ukuthi niyecansini nigaliqokile ijazi } \\
\text { lomkhwenyane }\end{array}$ & $\begin{array}{l}\text { Yebo } \\
(1)\end{array}$ & Cha \\
\hline 8. Wasebenzisa udlame ukuze niye ocansini & $\begin{array}{l}\text { Yebo } \\
\text { (1) }\end{array}$ & Cha \\
\hline $\begin{array}{l}\text { 9. Wasebenzisa udlame ukuze niye ocansini ngendlela } \\
\text { ezahlukile }\end{array}$ & $\begin{array}{l}\text { Yebo } \\
\text { (1) }\end{array}$ & Cha \\
\hline \multicolumn{3}{|c|}{ Total “Yebo" points: } \\
\hline \multicolumn{3}{|c|}{$\begin{array}{l}\text { To be IPV eligible: Must score } 2 \text { or higher on NVC or score } 1 \text { or higher in CTS- } \\
2 \quad \text { CTS-2: } \\
\text { NVC: }\end{array}$} \\
\hline \multicolumn{3}{|l|}{$\begin{array}{l}\text { IPV eligible } \\
\text { If yes, complete remainder of scales }\end{array}$} \\
\hline \multicolumn{3}{|c|}{$\begin{array}{l}\text { For those not IPV eligible: "Thank you very much for answering these } \\
\text { questions. We will not be in contact with you any further. Before I go, do you } \\
\text { mind if I quickly just ask what your age and race are for research purposes? } \\
\text { Age } \\
\text { Thank you." }\end{array}$} \\
\hline \multicolumn{3}{|c|}{ 4. VVS (put an X in each chosen block) } \\
\hline \multicolumn{3}{|c|}{$\begin{array}{l}\text { Lokhu okulandelayo kujwayele ukwenza kayingaki: } \\
\text { 0=Akujwayelekile/akusoza kwenzeke } \quad \mathbf{0 = N g o k u h a m b a ~ k w e s i k h a t h i ~}\end{array}$} \\
\hline
\end{tabular}




\begin{tabular}{|c|c|c|}
\hline \multicolumn{3}{|l|}{ 1=Njalo } \\
\hline $\begin{array}{l}\text { 1. Umlingani wakho uthatha izinto ozisho nozenzayo kabi noma } \\
\text { kunga fanele. }\end{array}$ & 0 & 1 \\
\hline $\begin{array}{l}\text { 2. Uma kukhona inkinga efuna ukhuthi niyixazulule, kuyashintsha } \\
\text { niqhudelane. }\end{array}$ & 0 & 1 \\
\hline 3. Uzizwa uwedwa kulobu budlelwane. & 0 & 1 \\
\hline $\begin{array}{l}\text { 4. Uma nixabana, oyedwa wenu aphonse ithawula...makwenzeka, } \\
\text { akasafuni ukukhuluma ngenxabano noma evele ehambe. }\end{array}$ & 0 & 1 \\
\hline 5. Uyamusaba umlingani wakho & 0 & 1 \\
\hline $\begin{array}{l}\text { 6. Uzizwa ngathi imizwa nemibono yakho ayibalulekile kolobu } \\
\text { budlelwane. }\end{array}$ & 0 & 1 \\
\hline $\begin{array}{l}\text { 7. Uma nixabana, umlingani wakho kufanele ephumelele futhi } \\
\text { akali laleli icala lakho. }\end{array}$ & 0 & 1 \\
\hline $\begin{array}{l}\text { 8. Ukewaba novalo nehloni ukwala izinto zo cansi zomlingani } \\
\text { wakho }\end{array}$ & 0 & 1 \\
\hline \multicolumn{3}{|l|}{ Total “Njalo” or Frequently points: } \\
\hline \multicolumn{3}{|l|}{ 5. HIRS (put an $\mathrm{X}$ in each chosen block) } \\
\hline \multicolumn{3}{|l|}{$\begin{array}{l}\text { Uyavumelana nalezizistetimende ezilandelayo? } \\
\text { Ngenxa yokuba nesifo senculaza... }\end{array}$} \\
\hline 1. Uzongena enkingeni nomlingani wakho & $\begin{array}{l}\text { Yebo } \\
(1)\end{array}$ & Cha \\
\hline 2. Umlingani wakho uzokuxasa & Cha (1) & $\begin{array}{l}\text { Yebo } \\
(0)\end{array}$ \\
\hline 3. Ubuhlobo benu buzohlukumezeka & $\begin{array}{l}\text { Yebo } \\
(1)\end{array}$ & Cha \\
\hline 4. Ukuthola imithi kuzoba nobungozi ngenxa yomlingani wakho & $\begin{array}{l}\text { Yebo } \\
(1)\end{array}$ & Cha \\
\hline 5. Uzozizwa ungaphephile ebuhlobeni benu & $\begin{array}{l}\text { Yebo } \\
(1)\end{array}$ & Cha \\
\hline $\begin{array}{l}\text { 6. Abanye bazokusiza uma uzizwa ungaphephile ebudlewaneni } \\
\text { benu }\end{array}$ & Cha (1) & $\begin{array}{l}\text { Yebo } \\
(0)\end{array}$ \\
\hline $\begin{array}{l}\text { 7. Akuphephile ukuxoxisana ngohlolwa kwengculaza } \\
\text { nomlingani wakho }\end{array}$ & $\begin{array}{l}\text { Yebo } \\
(1)\end{array}$ & Cha \\
\hline $\begin{array}{l}\text { 8. Akuphephile ukuxoxisana ngesimo sakho nesifo sengculaza } \\
\text { nomlingani wakho }\end{array}$ & $\begin{array}{l}\text { Yebo } \\
\text { (1) }\end{array}$ & Cha \\
\hline $\begin{array}{l}\text { 9. Uzizwa uphephile uma uthola amalanga ahloliwe } \\
\text { nabezomtholampilo }\end{array}$ & Cha (1) & $\begin{array}{l}\text { Yebo } \\
(0)\end{array}$ \\
\hline 10. Uzizwa uthukile ukuthi umlingani wakho uzokubulala & $\begin{array}{l}\text { Yebo } \\
\text { (3) }\end{array}$ & Cha \\
\hline
\end{tabular}




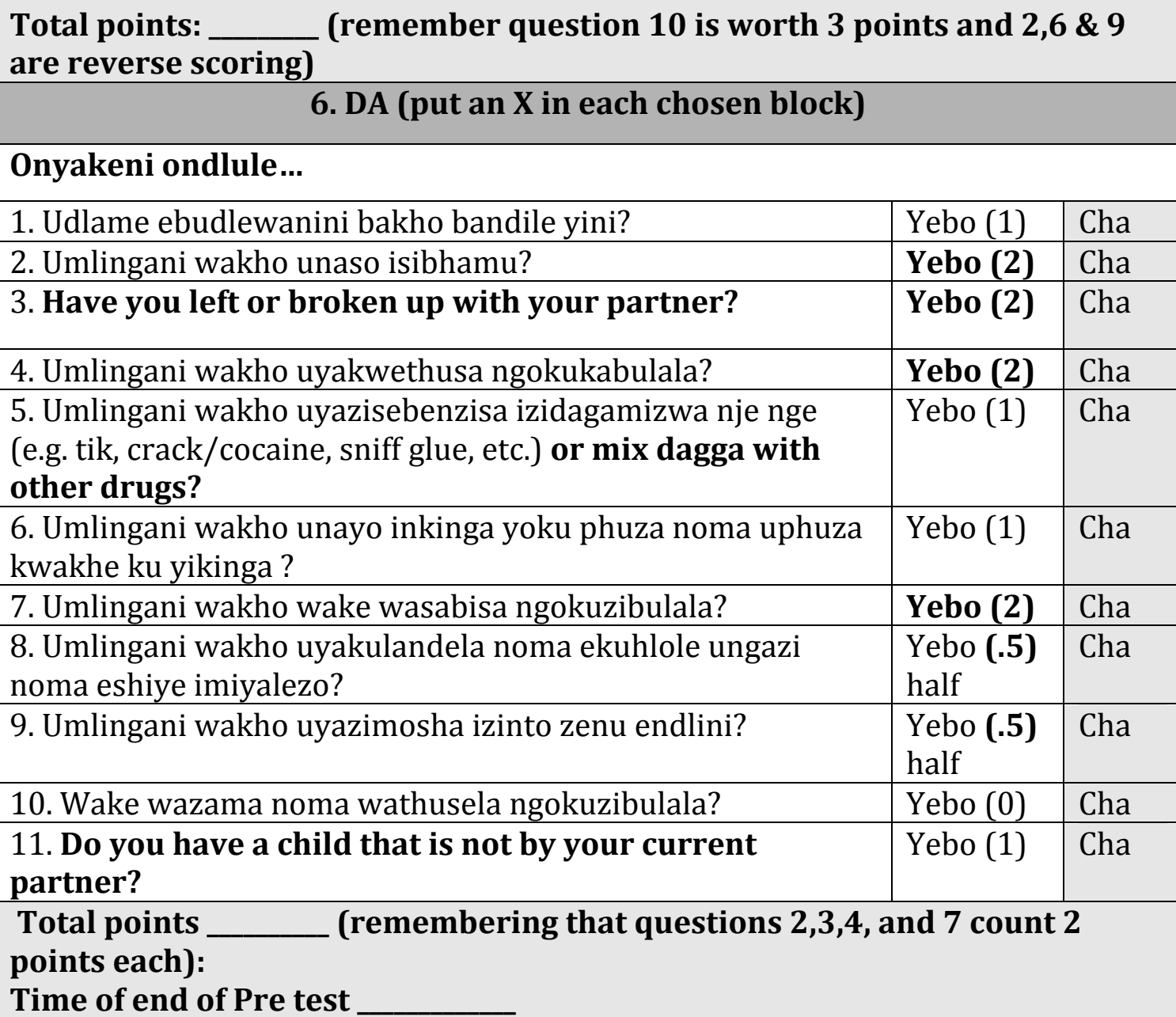

Thank you for answering those questions. Do you mind if I ask what your age and race are?

Race: Age:

Time of end of call:

For Standard of Care group, state:

Script: Thank you so much for participating. We will contact you again in one month."

For Experimental groups, tally their scores and begin Safety plan.

Totals:

NVC:

CTS-2:

VVS:

HIRS:

DA: 


\section{ONLY THOSE IN EXPERIMENTAL GROUP RECEIVE THEIR SCORE!}

Total Score:

$\begin{array}{lllll}\text { Meaning of Score: } & 1 & 0-5 & 6 & 26-30 \\ & 2 & 6-10 & 7 & 31-35 \\ 3 & 11-15 & 8 & 36-40 \\ & 4 & 16-20 & 9 & 41-45 \\ & 5 & 21-25 & 10 & 46-50\end{array}$


Appendix Q

\section{Safety Plans isiZulu}

Ngokwendlela ophendule ngayo imibuzo esitshelayo ngendlela ophephe ngayo ebudlelwaneni onabo, umphumela wakno ungu kokungu 10. Lapho u 10 asho ukuthi ubungozi buphezulu kakhulu.Uzizwa njani ngokuzwa lezindaba? (2)

Ulwazi lakho ngenculaza lusha futhi awukakabi nesikhathi esiningi sokucabanga. Kunga bandzima ukucabanga ukhuthi uzophepha kanjani ebudlelwaneni bakho njoba usuthole ulwazi. Nginolwazi olubalulekile. Abantu besimame bahlukunyezwa ngezindle ezintathu ebudlelwaneni: ngokomoya, ngokomzimba na ngokocansi. Konke lokuhlukunyezwa kungenyuka emva kokuthola ngesifiso senculaza. Izibonelo zokuhlukunyezwa ngokomoya ,ukubiza ngamagama amabi ,ukucikela phansi, ukuzama ukulawula .Izibonelo zokuhlukuyezwa ngokomomzimba ukushawa, ukhahlelwa noma ushayiwa ngempama. Izibonelo zokuhlukunyezwa ngokwecansi ukuphoqwa ukuya ecansini noma ukuphoqwa ukuthi ningali sebenzisi ijazi linkamkhwenyana . Abantu besimame abazithola ebudlelwaneni lapho kunodlame noku hlukumezeka, basencuphelweni enkulu uma sebethole ulwazi ngesimo sabo ngesifo sengculaza. Abanye abantu besifazane bazithola engozi enkulu uma beveza izimo zabo noma sebelanda imithi yesifo sengculazi. Abalingani babo bangazama ukubanqanda ukuthi bangawutholi umuthi noma bawuphuze .Abesimame abaningi bakhetha ukubatshela abalingani babo ukuzee nabo behlolwe futhi bewuthole umuthi. Uma ukhetha ukumtshela umlingani wakho, kuncono uthole icebo lokumtshela eliphephile.Yisinqumo sakho uma ufuna ukumshela umlingani wakho ngolwazi ngesifo sengculazi

Kulungile yini uma singakhuluma ngedlela zokuhlala uphephile ebudlelwaneni bakho?

19. Baninigi abantu empilweni yakho nasemphakathini abangakusiza uma uzizwa ngathi usengozini. Singabala udokotela wakho onesi, umeluleki, umfundisi.

LUkhona ucingo lamahala elinezimfihlo uma ufuna umuntu ozokhuluma naye noma udinga indawo yakulala. Inombolo eyodwa ithi: 0800-150-150. Kubalulekile ukubhala lokhu endaweni ephephile ukuze umlingani wakho angazukuyibona. Zikuphi izindawo eziphephile? (Isb: emsebenzini noma ocingweni lakho kodwa ubhale ngegama elinye). (3)

20. Abanye abantu babona kugcono ukukhuluma noma besizwe abantu ababaziyo. Lokhu kuchaza ukuthi uzokhulumo nelungu lomndeni, umngani noma ilungu lomphakhathi. Ukukhuluma nomdeni noma nomgani kusiza uma kunesimo esiphuthumayo 
> Cabanga ukuthi ubani emundenini noma kubangani ophephile (ongeke edlulise ulwazi kumlingani wakho). Ungamamuthinta lomuntu uma kunisimo esiphuthumayo (usebenzise igama noma isibonelo).

21. Lukhona nosizo olusemthethweni uma umlingani wakho enodlame. Ezinye izibonelo ukushayela amaphoyisa, ukuthinta umeli noma uhlelo lokuvimba ngomthetho (protection order).

$>$ Kahle kahle, uhlelo lokuvimba ngomthetho kuzovimbela umlingani wakho ukuze angangeni endlini yakho noma emusebenzini wakho, noma ukuthintana izingane zakho. Kanti futhi, loluhlelo lusiza ukuthintela okunye ukuhlukumezeka okungenzeka . Ungathanda ukwazi inombolo zokuthola ulwazi ngohlelo lokuvimba ngomthetho? (Kufanele ugcwalise amaphepha noma isitatimende esifugelwayo namaphoyisa asendaweni) noma ehovisi eliphezulu (ePetoli) shayela + 27 (0) 123931000 .

$>$ Gcina lokhu endaweni ephephile (ilungu lomndeni, kuwe endlini noma esikhwameni sakho noma phakathi kwerooge bese ubhala igama elihlukile eduze kwayo (ukudla, inkantini)

4. Abanye abantu bazama izinto ukuze benqande ukuhlukunyezwa ngeykhathi eziyingozi. Uma kuliwa, abanye abantu bazama ukupholisa abalingani babo, kodwa khumbula ukuthi ukuziphatha kwakhe akusikona icala lakho.

$>$ Zikhona izikhathi ongazicabanga ukuthi ungaqhelelana nazo ngoba ziyingozi khakhulu ?(4)

5. Abanye abantu bazama ukusinqaba isimo sokuhlukumezeka umasehla. Izibonelo zalokho ukuzilwelwa, ukufihla isibamu lapho kwazi wena wedwa, ukuzama ukuhamba noma uqede ubudlelwane, ukubaleka noma uzifihle ezikhathini. Uphenyo lukhobisa ukuthi ukunqanda ukuhlukunyezwa kunengozi futhi kwandisa udlame.

> Ucabanga ukushiya ubudlelwane bakho? (5) Yebo Cha Uma kunjalo, kungcono uzame icebo ngoba manje isikhathi esiyingozi kakhulu ebudlelwaneni obunodlame. (6) Cebo lithi

6. Amanye amasu ukugcina imali mhla kuvela isimo esiphuthumayo, inobolo ezibalulekile nezinto ongazidinga uma uhamba usushesha.

Ekuvaleliseni, ngingathanda ukukusiza wenze icebo ukuze uzizwe uphephile futhi uzinakekele, njoba uphethe ulwazi olusha ngobungozi bobudlelwane bakho. Uma usutshela umlingani wakho ngesimo sakho, iziphi izindlela ongazi phephela ngazo?(7)

Uma usola ukuthi kukhona okudla izithende, kubalulekile ukwazi ukuthi ukumbuza kunga letha olunye udlame, ukusebenzisa ijazi lomkhwenyana ngemuva kokuthola ulwazi ngesimo sakho kuyanciphisa uzalo lenculaza, kodwa ukucela umlingani wakho ukuthi esebenzise ijazi kunyusa udlame. Uma usaqala ukusebenzisa ijazi thola indlela ephephili yokumtshela. Kulungekakhuku emva kokuthola ulwazi ngesimo sakho ushone komtholampilo. Waze wanquma ukuthi ufuna ukutshela 
umlingani wakho ngesimo sakho, iyiphi indlela ephephile yokuthola imithi? Kufanele yini ukuthi umazise umlingani wakho uma uzophuma? Ungathini? 8)

Uma uCD4 cell count yakho iphansi (ngaphansi kwa 500), kuzofanela ukuthi uqale i antiretroviral theraphy medication. Indlela ephephile ejwayelekile ukufihla lemithi uma umlingani wakho enodlame. Eziphi ezinye izindlela zokwenza lokhu makudingeka? (Isibonelo, ukufaka amaphilisi ebhodleleni le-tylenol ( panado)/ noma uwagcine nawe). Ungenzani? (9)

Akukho okunye esingakusiza ngakho namhlanje ukuze uzizwe uphephile uthole imithi noma ukuze uphatheke kahle ngokomzimba nangokomoya?

\section{Time safety plan ends}

Qualitative answers from Safety Plan:

1. Level of danger is:

2. Feelings after hearing level of danger:

3. If accepted free hotline number, where is safe place to keep number:

4. Times/situations need to stay away from partner:

5. Yes or No (to leaving relationship)

6. Plan for leaving is:

7. Ways to protect self if discloses to partner:

8. Plan to safely get to HIV medical care or clinic:

9. Plan to keep HIV medication safe (if applicable):

10. Anything else we can help with to keep physically and emotionally safe: 
Appendix R

Post-test Experimental group isiZulu

Experimental

Post-test Time call begins

1. HIRS

Sukela uthola ulwazi ngesifo sengculaza ngawe... Uyavumelana nalezistetimente ezilandelayo?

\begin{tabular}{|c|c|c|}
\hline 1. Uke wangena enkingeni nomlingani wakho & \multicolumn{2}{|l|}{ Yebo } \\
\hline 2. Umlingani wakho uyakuxhasa & \multicolumn{2}{|l|}{ Yebo } \\
\hline 3. Ubudlelwane nomlingane wakho bubebucayi & \multicolumn{2}{|l|}{ Yebo } \\
\hline 4. Ukuthola imithi kube nengozi ngexa yomlingani wakho & \multicolumn{2}{|l|}{ Yebo } \\
\hline 5. Uzizwa ungaphephile ebudlelwaneni bakho & \multicolumn{2}{|l|}{ Yebo } \\
\hline 6. Abantu bakhona abakusizayo uma uzizwa ungaphephile & \multicolumn{2}{|l|}{ Yebo } \\
\hline $\begin{array}{l}\text { 7. Akuphephile ukuxoxa ngokuhlolwa kwengculaza nomlingani } \\
\text { wakho }\end{array}$ & \multicolumn{2}{|l|}{ Yebo } \\
\hline 8. Akuphephile ukuxoxa ngesimo senculaza nomlingano wakho & \multicolumn{2}{|l|}{ Yebo } \\
\hline $\begin{array}{l}\text { 9. Uzizwa uphephile uma uthola amalanga akhethiwe ukuze } \\
\text { uthole imithi }\end{array}$ & \multicolumn{2}{|l|}{ Yebo } \\
\hline $\begin{array}{l}\text { 10. Unokwethuka ukuthi umlingani wakho engazama } \\
\text { ukwebulala }\end{array}$ & Yebo & $\begin{array}{l}\mathrm{Ch} \\
\mathrm{a}\end{array}$ \\
\hline \multicolumn{3}{|l|}{$2.5 S$} \\
\hline \multicolumn{3}{|l|}{ Uyavumela nalokhu okulandelayo: } \\
\hline 1. Kukokonke, ulwazi olutholile libenosizo kakhulu & $\begin{array}{l}\text { Yeb } \\
0\end{array}$ & Cha \\
\hline 2. Kube nosizo kakhulu ukubuzwa ngezinkinga zobudlelwane bami & $\begin{array}{l}\text { Yeb } \\
0\end{array}$ & Cha \\
\hline $\begin{array}{l}\text { 3. Ufisa ngathi ngabe nixoxe kabanzi ngezinkinga zobudlelwane } \\
\text { bami }\end{array}$ & $\begin{array}{l}\text { Yeb } \\
0\end{array}$ & Cha \\
\hline 4. Bekungangihlalisi kahle ukuxoxa ngezinkinga zami & $\begin{array}{l}\text { Yeb } \\
0\end{array}$ & Cha \\
\hline 5. Uyazisola ngokukhuluma ngekinga zobudlwane bami & $\begin{array}{l}\text { Yeb } \\
0\end{array}$ & Cha \\
\hline 6. Ukukhuluma ngekinga zama kungi beke ebungozini obukhulu & $\begin{array}{l}\text { Yeb } \\
0\end{array}$ & Cha \\
\hline
\end{tabular}




\begin{tabular}{|c|c|c|c|c|}
\hline \multicolumn{4}{|l|}{ 7. Usizo olutholile lokubeke ebungozini obukhulu } & Cha \\
\hline \multicolumn{5}{|l|}{ 3. FAS } \\
\hline 1. Ulisebenzisile icebo lokuphepha? & \multicolumn{2}{|l|}{ Yebo } & \multicolumn{2}{|c|}{ Cha } \\
\hline 2. Lelicebo ulithole linosizo olukhulu? & \multicolumn{2}{|l|}{ Yebo } & \multicolumn{2}{|c|}{ Cha } \\
\hline \multicolumn{5}{|l|}{ 3. Iliphi uso olusebenzise kakhulu? } \\
\hline \multicolumn{4}{|c|}{$\begin{array}{l}\text { A. Ukukhuluma nodokotela, unesi nomunye umuntu osebenza } \\
\text { kwamtholampilo }\end{array}$} & Yebo \\
\hline \multicolumn{4}{|c|}{ B. Ukukhuluma nomululeki } & Yebo \\
\hline \multicolumn{4}{|c|}{ C. Ukukhuluma noclergy, nomfundisi/spiritual leader } & Yebo \\
\hline \multicolumn{4}{|c|}{ D. Ukubeka usizo nabo bedlame lwasendlini (ngocingo,uyele, ibandla) } & Yebo \\
\hline \multicolumn{4}{|l|}{ E.Ukukhuluma nomndeni noma umngani } & Yebo \\
\hline \multicolumn{4}{|c|}{$\begin{array}{l}\text { F. Thintana namaphoyisa, umeli noma uthola uhlelo lokuvimba } \\
\text { ngomthetho }\end{array}$} & Yebo \\
\hline \multicolumn{4}{|c|}{$\begin{array}{l}\text { G. Uzama ukubalekela udlame (ngenye iynkathi ngamboni umlingani, } \\
\text { wenze lokhu umlingani whakho ethi kwenze) }\end{array}$} & Yebo \\
\hline \multicolumn{4}{|c|}{ H. Ukuzilwela, uqede ubudlelwane, ukubaleka ucashe uma udlame luqala } & Yebo \\
\hline \multicolumn{4}{|c|}{$\begin{array}{l}\text { I. Ukucababgela phambili njengokufihla imali, ukugcina izinombolo } \\
\text { ezibalulekile }\end{array}$} & Yebo \\
\hline \multicolumn{4}{|c|}{ J. Ukuthola amasu okuya komtholamphilo utho yenculaza } & Yebo \\
\hline \multicolumn{4}{|c|}{ K. Ukuthola indlela ephephile yokuthola imithi njalo nje } & Yebo \\
\hline $\begin{array}{l}\text { 4. Ungaphakamisa icebo lokuphepha } \\
\text { abanganini bakho abanekinga efana neyakho? }\end{array}$ & Yebo & \multicolumn{3}{|l|}{ Cha } \\
\hline $\begin{array}{l}\text { 5. Kungaphepha uma singakulandela ngocingo } \\
\text { emva kwe... }\end{array}$ & $\begin{array}{l}\text { Nyan } \\
\text { ga } 1\end{array}$ & $\begin{array}{l}\text { Nyang } \\
\text { a } 2\end{array}$ & $\begin{array}{l}\text { Nyanga } \\
3\end{array}$ & \\
\hline \multicolumn{2}{|c|}{$\begin{array}{l}\text { 6. Sukela uthola ngesifo senculaza, usuke waya } \\
\text { kwomatholampilo wathola usizo (uthole imiphumela } \\
\text { ye CD4 cell, wakhuluma nomemuleki)? } \\
\text {-If so, when did you go (exact date): }\end{array}$} & \multicolumn{2}{|l|}{ Yebo } & Cha \\
\hline \multicolumn{2}{|c|}{$\begin{array}{l}\text { 7. Uma usumazisile umlingani wakho ngesimo sakho, } \\
\text { ubone udlame (ngokomoyo, ngokomzimba noma } \\
\text { ngokwecansi)? }\end{array}$} & \multicolumn{2}{|l|}{ Yebo } & Cha \\
\hline
\end{tabular}

Time call ends 
Appendix S

Post-test Standard of Care isiZulu

\begin{tabular}{|c|c|c|c|c|}
\hline \multicolumn{4}{|l|}{ Post-test } & \\
\hline \multicolumn{5}{|c|}{ 1. HIRS } \\
\hline \multicolumn{5}{|c|}{$\begin{array}{l}\text { Kumuva kokuthola ulwazi ngawe nesifo senculaza ...Uyavumelana } \\
\text { nalezistetimente ezilandelayo? }\end{array}$} \\
\hline \multicolumn{3}{|c|}{ 1. Ukewaba nekinga nomlingani wakho } & Yebo & Cha \\
\hline \multicolumn{3}{|l|}{ 2. Umlingani wakho uyakuxhasa } & Yebo & Cha \\
\hline \multicolumn{3}{|c|}{ 3. Ubudlelwane benu buele bababucayi } & Yebo & Cha \\
\hline \multicolumn{3}{|c|}{$\begin{array}{l}\text { 4. Ukuthola ukulapheka kobe nokungozi ngenxa yomlingani } \\
\text { wakho }\end{array}$} & Yebo & Cha \\
\hline \multicolumn{3}{|c|}{ 5. Uzizwa ungakhoselekile ebudlwelwane benu } & Yebo & Cha \\
\hline \multicolumn{3}{|c|}{$\begin{array}{l}\text { 6. Abanthu bakhona abakusekelayo uma uzizwa } \\
\text { ungakhoselekile ebudlelwaneni bakho }\end{array}$} & Yebo & Cha \\
\hline \multicolumn{3}{|c|}{$\begin{array}{l}\text { 7. Akuphephile yini uma ukhuluma ngokuhlolwa isifo senculaza } \\
\text { nomlingani wakho }\end{array}$} & Yebo & Cha \\
\hline \multicolumn{3}{|c|}{$\begin{array}{l}\text { 8. Akuphephile uma nixoxa ngesimo sakho mayelana nesifo } \\
\text { senculaza nomlingani wakho }\end{array}$} & Yebo & Cha \\
\hline \multicolumn{3}{|c|}{$\begin{array}{l}\text { 9. Uzizwa uphephile uma unqumelwa usuku lokuya } \\
\text { kotholampilo }\end{array}$} & Yebo & Cha \\
\hline \multicolumn{3}{|c|}{ 10. Uzizwa unoalo ukhuthi umlingani wakho engakubulala } & Yebo & Cha \\
\hline \multicolumn{5}{|c|}{ 2. SS } \\
\hline \multicolumn{5}{|c|}{ Uyavumelana nalezistetimente ezilandelayo: } \\
\hline \multicolumn{3}{|c|}{ 1. Kukokonke, ulwazi olutholile ngobudlwelwane libonosizo } & Yebo & Cha \\
\hline \multicolumn{3}{|c|}{ 2. Kubenosizo ukubuzwa ngobunzima abutholakala ebuhlobeni } & Yebo & Cha \\
\hline \multicolumn{3}{|c|}{ 3. Ufisa ngathi uxoxisane kabanzi ngobunzima ebuhlobeni bakho } & Yebo & Cha \\
\hline \multicolumn{3}{|c|}{$\begin{array}{l}\text { 4. Bekumgangi phathi khahle uma ukhuluma ngekinga } \\
\text { ebuhlobeni bakho }\end{array}$} & Yebo & Cha \\
\hline \multicolumn{3}{|c|}{ 5. Uyazisola ngokuxoxa ngezikinga zobudlelwane bakho } & Yebo & Cha \\
\hline \multicolumn{3}{|c|}{ 6. Ukuxoxa ngekinga zabudlewano bakho beke wena engozini } & Yebo & Cha \\
\hline \multicolumn{3}{|c|}{ 7. Usizo olitholile likubeke engozini enkulu } & Yebo & Cha \\
\hline $\begin{array}{l}\text { 8. Kungaba ncono uma sikuthinte } \\
\text { ngomakhalekhukhwini... }\end{array}$ & Inyanga 1 & Inyanga 2 & \multicolumn{2}{|c|}{ Inyanga 3} \\
\hline \multicolumn{3}{|c|}{$\begin{array}{l}\text { 9. Usuwaya yini etholampilo uyothola imithi ngemva kokuthola } \\
\text { ulwazi ngawe ngesifo senculaza (imphumela ye CD4 cell, } \\
\text { ukukhuluma nomeluleki ngamakhambi athize)? } \\
\text {-If so, when did you go (exact date): }\end{array}$} & Yebo & Cha \\
\hline \multicolumn{3}{|c|}{ 10. Uma usumtshelile umlingani wakho ngesimo sakho, ubone } & Yebo & Cha \\
\hline
\end{tabular}


ushintso ebudlelwaneni benu? (ngokomzimba, ngokomqondo noma ngokocansi)?

11. Enyangeni endlule, ukewathola usizo mayelana nokuhlukumezeka ebudlelwane bakho kulezi ndawo: emphoyiseni, ucingo lokuhlukumezeka endlini noma uhlelo umeluleki, umfundisi noma ezinye izindawo 
Appendix T

\author{
Informed Consent form Sesotho
}

\title{
Subject Informed Consent Document
}

\section{HIV Screening and Intimate Partner Violence Safety Study}

Sponsor(s) name \& address:University of Louisville, Louisville, KY and Shout-itNow, Cape Town, SA

Investigator(s) name \& address: Primary Investigator Michiel A. van Zyl and Coinvestigator Leslie Lauren Brown: 2217 South Third St. Louisville, KY 40292, USA.

Site(s) where study is to be conducted: Gauteng province and telephone calls will be made from Cape Town, South Africa.

Phone number for subjects to call for questions:021 7134414

\section{Introduction and Background Information}

O memelwa ho nka karolo diphuputsong. Dr. Riaan van Zyl, PhD;Lauren Brown, LCSW, PhD Candidate YA Shout-it-Now. Diphuputso di patallwa ke University of Louisville, lefapheng la Kent School of Social Work. Thupelo etla nka sebaka di dibakeng tsohle tsa bohlabolli le diteko tsa HIV proincing ya Gauteng ka nako ya thupelo tsena. Diphuputso di tla nka sebaka ka nako ya thupelo. Dipuisano di tla etswa ka mohala ka Shout-it-Now call centre e sebakeng sa Tokai, Western Cape. Bonyane batho ba 225 ba tla memelwa ho nka karolo.

\section{Sepheo}

Sepheo thutong ena ke ho bona hore basadi ba kgola ho tseba hore lerato la bona le lehlakoreng la polokeho ho hlahiswa ha HIV kapa hoba le leqheka le bolokehileng la mora diteko

\section{Tsamaiso}

Mo thutlong ena otla hoka hangwa ka mohala letsatsi kamora ho bona tshebediso ka pitso ya hona jwale. Honka karolo e ka etsahala ka seka sele seng kanako tse kabang pedi.

Haole monka karalo ya seka sa pele sa thuto, teko ya mohala kappa mokgahlo. Oo shebileng otla o boning otla sebediswa feela ho thusa ho lokafatsa tholo ya thuto kappa hokenella (dikahare tsa puisano ya mohala). Retla holahana le wena 
ho bona tshwaelo ya hao ka dipotso tsa tokafatso le diteng tsa puisano ya mohala. Re batla ho tseba hore o nahana hore dipotso tsena ditla re tswela masola ho inwela, ho o etsa oseka wa dudiseha kapa ho hlolehala ho fetola hore batho ba utlwisise haholo dipotso kapa dipuisano le ho ikutwa ba kgotsofaditswe ke tsamaiso tse ding tsa dipotso kapa dipuisana le ho ikutwa ba kgotsofaditswe ke tsamaiso tse ding tsa dipotso kapa dipotso kaofela hotswa ho seka seo. Thodiso ena kapa mokgahlo eka sehatiswe empa mo boemong dipatlisiso tsa hao ditla ngolwa fatshe ka letshoho kapa e hatiswe ka mochini wa botaki haole monka karolo moo sekeng sena sa bobedi sa thuto o tla botswa ho thusa ho bontsha thireletso ya hao setwalleng sa dikamano. Dipotso tse ding di fela hore ruta le dipotso tse ding di tswella ho botsiswa moo dikarolong tse di fapanang tsa lefatshe ho batlisisa tshireletso ya setswalle. Dipotso tse ding di amana le popeho kapa bongwe ba kgolahano le molekana le tse ding tsa tshireletso ya maikutlo hona le dipotso hape tse mmalwa ka maitemohelo ao o ka bang o a bone moo nakong e fetileng ka moratuwa oo utlwisang bohloko. O tla hoka hangwa ha bedi kapa ha raro ka thuto le ho botswa dipotso ka nako ya hokahanyo ya pele le ya hoqetela. Dipotso tsa pele ka kakaretso di tshwanetse ho tsamaya metsotso e 8-20 hore di arabuwe. Hone le dipotso tse kgutsafaditsweng dile 46 tsa kgokahano ya pele le dipotso tse kgutsofaditsweng dile 18-30 tsa kgolahano ya hoqetela. Ha ho kaba le dipotso tseodi o etsang o seke wa dudiseha oka kgetha ho se diarabe. O ka tswella ho amohela tshebediso ya S-N le ha o sa araba tse ding kapa dipotso kaofela. Banka karolo ba seka sa bobedi sa thuto e ba tsweletsa ho thuta ho mekgatlo efapaneng hore seka ban ka karolo bohle ba amohela tshebediso tse tshwanang.

\section{Kotsi e bohlokwa}

Thutong ena e na le karolo ya tse ding tsa boiteko bo bongata a hlahellang moo ho itlatlhobong HIV le bao ba setseng ba tshwaeditswe ke baratuwa ba bona ba setseng ba tshwaeditswe ke baratuwa ba bona ba ba utlwisang bohloko (ka thuto kapa eseng ka thuto) . diteko tse ding tsee din ka karolo ka diteko tsa HIV kapa o ikutlwa o utlwile bohloko ka nako ya teko ya HIV e ka oketsehang ha motho a bolellwa hore ona le HIV baka ikutlwa ba tshohile kapa ba ikgatholla kapa ba a hlolwa le leloko le setjhaba o ka ba kotsing e feteletseng ya ho utlwiswa bohloko haho setse hona le le mong le moratuwa o ho utlwisang bohloko moo leratong la lona. Ka hoba kutlwiso bohloko ke moratuwa moo leratong eka oketseha ha omong a fihletswe a le HIV kapa leka holo amohela kalafi ya HIV .

Bokotsi ba thuto ena ke kgonahalo ya ho ikutlwa osa phutholoha hao araba dipotso tse thatha ka bowena, hona ho ikekellwa ha thobalana ho araba dipotso tsena hoka o bakela ho hoopla di ketsahalo otla fuwa tsebiso ya mosebetsi ya haufi yaka o thusang. Khotsi engwe e bohlokwa ka thuto ena ke ho o eletsa jwale o ka sebedisa mohala ho ikopanya le wena. Sena se kaba kotsi ha moratuwa wa hao a ka lemoha hore o amohetse mohala oo. O seke wa araba mohala ha ho sa loka, o seke wa etsa jwalo. O ka ikopanya le lefapha la mehala ha ho loketse ho etsa jwalo, hobane re tlo leka ho o fihlella ka nako e ngwe, ho letsa homa jwaele 
etsa e etse ka mokgwa o mong wa ho letsa ha morao, ebile ke matsapa a feng a beilweng.

Ditlhatlhobo di behilwe hore tshebediso ya basadi ba ntshwang kotsi mo leratong ya basadi ba ntshwang kotsi mo leratong la bona. Hahole jwalo ditholwana tsa thato ena hlahella hoba ka hodimo ha kotsi.

\section{Kgolo}

Thuto ena le dikgolo tse bohlokwa. O ka itemohela maikutlo eya theilweng ka ho feteletseng kapa ho ba le disebediswa e le ditla morao tsa ho nka karolo moo thutong ena. O ka fihlela hore dipotso tse dika o thusa ho nahana ka kamahano ya hao leho batla thuso lelokong kapa setjhabang hore o sireletsehe. Ba nka karolo ba fihletsweng bana le HIV ke tshepo ya hore batla kgona ho bona kalafo ya HIV e sireletsehileng. Kaho fitisisa ho fitisa baneng ba sa nke karalo thutong ena. Dipatiisiso di bontsha hore basadi ba bang ba nka karolo dithutong ka baratuwa ba bona bao utlwisang bohloko bare ba fihletse thuto ena. Ba oketsa tsebo ya bona ka tsebiso ya hotswa ho thuto etla etswa ho thusa basadi ba bang ho fihlele tshebediso ya HIV ka mokgwa o sireletsehileng. Ho fihlella tshebediso ya HIV tshireletso ho basadi ba ka ikutwa ba kgotsofetse hofitisisa ka maphelo a bona hape le ka maitemohelo a eketsehileng a tsa maphelo kapa ho phela nako etelele. Qetellong maitemohelo a ka ke hore palo ya batho ba ba tshwaetsang ba bang HIV hape palo ena emyame ya batho ba shwa ka baka la HIV .Tsebo ena ya thuto eka se ho kgole re ithutile hore thuto ena eka thusa ba bang. .

\section{Papiso}

Hao nka karolo thutong ena, hape o sala ka hara thato ena kaofela, lebitso la hao letla Kenya thodisanong ya kgalo ya i-pod ya mahala. Kemyeletso ya ba nkileng karalo thutong otla amohela HIV /TB thatlhobo le kemoso le thoso ya ho hlahlobelwa moratuwa wa hao mahala. Hao sa battle ho nka karolo thutong, otla amohela thatlhobo ya HIV/TB le kemoso empa ho kenyeletswe moratuwa wa hao ya o hlekefetsang.

\section{Lekunuthu}

Sephiri seka se tshepuwe. Sehiri sa hao setla bolokeha ho fihlela tumellanong sa molao. Haeba sepheto sa thuto se phatlaladitswe, lebitso la hao leka se phatlalatswe. Haho se jwalo, ba latelang batla lekola rekota ya thuto. Setheo sa thuto ekgolo sa Louisille sa lekala la thatlhobiso kantoro le kantoro e ikgathileng The University of Louisville Institutional Review Board, Human Subjects Protection Program Office, and Privacy Office. Office for Human Research Protections (OHRP)

Taba ka ngwe etla amohela nomoro hore lebitso la hao le se sebediswe ho hlalosa ho tsejwa ha hao data (kapa tsebo hotswa thutong) etla behwa ke ba hlahlobi ba lekgotla la Africa Borwa. Hang ha thuto e qetilwe, tsebo ya thuto (data) o hlodilwe hotsha dinomorong eseng mabitsong. Etla romelwa ka email 
hoba hlahlubi ba tsa thuto ena. TIhebo ka computa etlaba HIPAA ka botlalo (ho latela lefapha la tsa Health Insurance Portability and Accountability Act). Defile otla dithola ka cabineteng e notletsweng ya primary officer.

\section{Baithaupi}

Honka karolo thutong ena ya baithaupi. O ka kgetha ho senke karalo hohong. Hao nka qeto ya hoba thutong ena oka emisa monka karolo nako efe kapa efe hao emisa honka karalo nako engwe le engwe, o ka se lahlehlwe ke dikqolo tsa seo tshwanetseng.

\section{Patlisiso ya tsa Dilokelo, Dipotso, Tumelano le Ditlotlebo}

Patlisiso ya tsa dilokelo, dipotso, tumelano le ditletlebo.

Hao na le seo osa se utlwisising kapa seo o tletlebang ka sona ka thato ena kapa disebediswa tsa thuto ema le dikgetho tse tharo.

O ka hokahana ka mohala le Mosuwe hloho ya dipatlisiso ho 502-852-2430 (USA).

Haeba ona le dipotso ka ditokela tsa hao ka ha thuto, dipotso, le hoba kgahlano, kapa hotletleba o ka hoka hana le ba kantoro ya Human Subjects Protection Program Office (HSPPO) (502) 852-5188. O ka bua ka dipotso kaha ditokelo tsa hao ka hara sephiri, le setho sa setheo sa thuto e kgalo kapa kantoro ya Institutional Review Board (IRB) kapa ba HSPPO. IRB e hlahisitswe thusong ena

Haiba o batla ho bua le motho kantle ho lefapha la thutelo, oka hokahana le 187-852-1167. O ka fuwa monyetla wa ho bua ka dipotso, kgahlano kapa tletlebo sephiring. Sena ke 24 1-87-852-1167. O ka fuwa monyetla wa ho bua ka dipotso, kgahlano kapa tletlebo sephiring. Sena ke 24 a dihora ya mohala o arajwang ke batho basa sebetseng lefaphang University of Louisville.

Pampiri ena yao bolella hore ho etsahalang nakong eo o kgethang honka karalo. Ho tekema ho bolela hore thuto ena ho buisana le wena hore dipotso tsa hao di arabilwe, hape o tla nka karalo thutong ena. Sena hase tumellano. Hao nehelane ka ditikelo tsa molao ka ho tekema lengolo lena o tla fuwa pampiri ya bopaki hoe beya ele bopaki. 
Gatisa lebitso la motho

English
isiZulu

Xhosa

Sesotho

Nomoro ya mogala watho:

Tekeno ya moemedi wa Moloa Kekeno ya letsatsi

Motho ya Hlalosang Lemgolo la Kumellano (if other than the Investigator)

Tekenoya Mmatiisisi

Kekano ya letsasi

LENANE LA BABATIISISI NOMORO YA MOGALA

Michiel A. van Zyl, PhD

$1-502-852-2430$

Lauren Brown, LCSW, PhD Candidate 1-502-852-2430 
Sesotho Pre-test

Script: "Hi, this is with Shout-it-Now. I was given your information from my shout-it-now teammate because you agreed to participate in a brief study with us. This will take about 20 minutes or more. Are you still willing to participate in this study? Great. Is this a safe time to talk? okay, I am going to ask you some questions now."

\begin{tabular}{|c|c|c|}
\hline \multicolumn{3}{|l|}{ Pre-test } \\
\hline \multicolumn{3}{|l|}{ 1. RS } \\
\hline 1. Anai o maratong & Nnete & Tjhee \\
\hline $\begin{array}{l}\text { 2. (Ha hole jwalo, se arebe potso ya bobedi): Ha ngata o ithola } \\
\text { ole maratong }\end{array}$ & Nnete & Tjhee \\
\hline \multicolumn{3}{|c|}{ 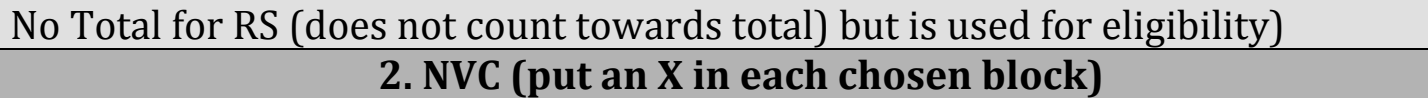 } \\
\hline \multicolumn{3}{|l|}{ o kare ho moratuwa wa hao... } \\
\hline 1. Leka ho fokotsha kamano ya leloko la hao le metswalle? & Nnete (1) & Tjhee \\
\hline 2. Ke mona kapa ho o laola? & Nnete (1) & Tjhee \\
\hline 3. 0 phehella ho tseba hore ona lemang ka nako tsohle? & Nnete (1) & Tjhee \\
\hline 4.0 o bitsa ka mabitso kappa oo nkela fatshe pela ba bang? & Nnete (1) & Tjhee \\
\hline 5. 0 etsa hore o ikutlwe o sena loleng kapa mosola? & Nnete (1) & Tjhee \\
\hline 6. Wao halefela kappa ho ho hlapaola? & Nnete (1) & Tjhee \\
\hline 7.0 utlwisitse mmele wa hao bohloko? & Nnete (1) & Tjhee \\
\hline \multicolumn{3}{|l|}{ Totala "Nnete" (or Yes) points: } \\
\hline 3. CTS-2 (put an X in each chosen blo & & \\
\hline \multicolumn{3}{|l|}{ Ka selemo sefitileng moratuwa wa hao... } \\
\hline 1. 0 o shapile? & Nnete (1) & Tjhee \\
\hline 2. 0 o shapile ka hohong? & Nnete (1) & Tjhee \\
\hline 3.0 o kgamme? & Nnete (1) & Tjhee \\
\hline 4. Oho shapile haholo? & Nnete (1) & Tjhee \\
\hline 5.0 o tshoseditse ka sebetsa? & Nnete (1) & Tjhee \\
\hline
\end{tabular}




\begin{tabular}{|c|c|c|}
\hline 6. O sebedisetse sebetsa ho wena? & Nnete (1) & Tjhee \\
\hline 7. 0 entse hore le etse thobalano ntle le tshireletso & Nnete (1) & Tjhee \\
\hline 8. 0 o hatelletse hore le etse thobalano? & Nnete (1) & Tjhee \\
\hline $\begin{array}{l}\text { 9. } 0 \text { hatelletse hore le etse thobalano ya pele kapa ya ka } \\
\text { morao }\end{array}$ & Nnete (1) & Tjhee \\
\hline \multicolumn{3}{|c|}{ Totala “Nnete" (or Yes) points: } \\
\hline \multicolumn{3}{|c|}{$\begin{array}{l}\text { NVC: } \quad \text { CTS-2: } \quad \text { Total: } \\
\text { IPV eligible } \quad \text { Yes }- \text { No } \\
\text { If ves, complete remainder of scales }\end{array}$} \\
\hline \multicolumn{3}{|l|}{ 4. VVS (put an X in each chosen block) } \\
\hline \multicolumn{3}{|l|}{$\begin{array}{l}\text { O kare tsena dietsehala kgafetsa hakakang? } \\
\text { Ha ho kgonahale, ka haho etsahale Ha ngwe ka mora nako } \\
\text { Kgafetsa }\end{array}$} \\
\hline $\begin{array}{l}\text { 1. Moratuwa wa hao o hlahisa mantswe a hao kapa diketso ka } \\
\text { mokgwa o sa nepahalang ho feta ka moo o di bolelang. }\end{array}$ & 0 & 1 \\
\hline $\begin{array}{l}\text { 2. Ha lena le mathata ho o rarolla, o kare lo mokgahlo o } \\
\text { fapaneng. }\end{array}$ & 0 & 1 \\
\hline 3. O ikutlwa ole mong se tswalleng sa hao & 0 & 1 \\
\hline $\begin{array}{l}\text { 4. Hale ngangisana, o mong o tshe-tshella morao, ka seo, ha a } \\
\text { sabatla ho bua ka sona kapa o tlohele taba ele jwalo. }\end{array}$ & 0 & 0 \\
\hline 5. O tshaba moratuwa wa hao. & 0 & 0 \\
\hline $\begin{array}{l}\text { 6. O oikutlwa ekareng tlhahiso kapa maikutlo a hao ha a nataba } \\
\text { setwalleng sa hao? }\end{array}$ & 0 & 0 \\
\hline $\begin{array}{l}\text { 7. Hale ngangisano moratuwa wa hao o tlameha ho hlola } \\
\text { ngangisaho hape aka se mamele lehlakore la taba ya hao. }\end{array}$ & 0 & 0 \\
\hline $\begin{array}{l}8.8 \text { o ba le ho tshoha kapa ho tshaba ho hana ha morutuwa oa } \\
\text { hao aleka hore le etse thobalano. }\end{array}$ & 0 & 0 \\
\hline \multicolumn{3}{|l|}{ Totala “Kgafetsa” (or Frequently) points: } \\
\hline \multicolumn{3}{|l|}{ 5. HIRS (Put an X in each chosen block) } \\
\hline \multicolumn{3}{|c|}{$\begin{array}{l}\text { O dumellana le sebolewang? } \\
\text { Ka baka la boemo ba ha obo kwaysi ya bosalla tlhapi HIV ... }\end{array}$} \\
\hline 1. O tlaba ka hara mathata le moratuwa wa hao & Nnete (1) & Tjhee \\
\hline 2. Moratuwa wa hao e tlaba tshebetso ho wena & Tjhee (1) & Nnete(0) \\
\hline $\begin{array}{l}\text { 3. Setswale sa hao le moratuwa wa hao setlaba ma emong } \\
\text { a hlobaetsang haholo na? }\end{array}$ & Nnete & Tjhee \\
\hline $\begin{array}{l}\text { 4. Ho fumana pheko ho thlaba kotsi ka baka la moratuwa } \\
\text { wa hao }\end{array}$ & Nnete & Tjhee \\
\hline 5. 0 tla ikutlwa osa bolokeha ka hara setswale la lona & Nnete & Tjhee \\
\hline
\end{tabular}




\begin{tabular}{|c|c|c|}
\hline $\begin{array}{l}\text { 6. Babang batla o thusa ha o ikutlwa osa tshirelletseha se } \\
\text { tswaleng sahao }\end{array}$ & Tjhee (1) & Nnete(0) \\
\hline $\begin{array}{l}\text { 7. Ha wa bolokeha hao bua ka boemo bo hao ba kwatsi ya } \\
\text { bosolla tlhapi HIV le moratuwa wa hao }\end{array}$ & Nnete & Tjhee \\
\hline $\begin{array}{l}\text { 8. Ha hao bolokeha ho bua ka boemo ba hao ba kwatsi ya } \\
\text { bosolla thapi HIV le moratuwa wa hao }\end{array}$ & Nnete & Tjhee \\
\hline $\begin{array}{l}\text { 9. } 0 \text { i kutlwile o bolokehile hao kena ho tsa hlophiso ya } \\
\text { maphelo? }\end{array}$ & Tjhee (1) & Nnete(0) \\
\hline 10. 0 tshaba moratuwa wa hao otla leka hoo bolaya & $\begin{array}{l}\text { Nnete } \\
\text { (3) }\end{array}$ & Tjhee \\
\hline \multicolumn{3}{|c|}{$\begin{array}{l}\text { Totala points: } \\
9 \text { are reverse scoring) }\end{array}$} \\
\hline \multicolumn{3}{|c|}{ 6. DA (put an X in each chosen block) } \\
\hline \multicolumn{3}{|l|}{ Ka selemo sa ho se fitileng ... } \\
\hline 1. Moferefere setswaleng saho oile wafitisisa & Nnete (2) & Tjhee \\
\hline 2. Moratuwa wa hao ona le sethuya? & Nnete (1) & Tjhee \\
\hline 3. 0 mmoho le moratuwa wa hao? & Nnete (1) & Tjhee \\
\hline 4. Moratuwa wa hao o tshosetsa ho o bolaya? & Nnete (2) & Tjhee \\
\hline $\begin{array}{l}\text { 5. Moratuwa wa hao o sebedisa di thitefatsi tse seng } \\
\text { molaong jwaloka (e.g. tik, crack/cocaine, sniff glue, } \\
\text { etc.)kappa ho kopanya matekwane mmoho le bojwala kappa } \\
\text { tse ding tsa dithethefatsi? }\end{array}$ & Nnete (1) & Tjhee \\
\hline $\begin{array}{l}\text { 6. A na moratuwa wa hao ke letawa kappa onale bothata ba } \\
\text { honwa }\end{array}$ & Nnete (1) & Tjhee \\
\hline 7. Ebe moratuwa wa hao oile a tshosetsa ho I polaya. & Nnete (2) & Tjhee \\
\hline $\begin{array}{l}\text { 8. Moratuwa wa hao o waho latela kapa ahonyonyobele kapa } \\
\text { a siye melaetsa etshosetsang }\end{array}$ & Nnete (1) & Tjhee \\
\hline 9. Moratuwa wa hao oile a senya thapa ya hao? & Nnete (1) & Tjhee \\
\hline 10. O kile wa tshosetsa kapa ho leka ho ipolaya? & Nnete (2) & Tjhee \\
\hline
\end{tabular}

Thank you for answering those questions. Do you mind if I ask what your age and race are?

Race: Age:

For Standard of Care group, state:

Script: Thank you so much for participating. We will contact you again in one month."

For Experimental groups, tally their scores and begin Safety plan.

\section{Totals:}

NVC:

CTS-2: 
VVS:

HIRS:

DA:

ONLY THOSE IN EXPERIMENTAL GROUP RECEIVE THEIR SCORE!

Total Score:

Meaning of Score

$10-5$

$2 \quad 6-10$

$3 \quad 11-15$

$4 \quad 16-20$

$5 \quad 21-25$

$\begin{array}{ll}6 & 26-30 \\ 7 & 31-35 \\ 8 & 36-40 \\ 9 & 41-45 \\ 10 & 46-50\end{array}$




\section{Appendix V}

Sesotho Safety Plan

Moloo o ngotsweng wa Tshireletso:

Ho ea ka dikarabo tseo ore nehileng tsona,tse re bolellang hore o tshereletsehile ha kakang setswaleng sena sa hao.O fomane dintlha tse ho tse 10.mo leshume (10) le bolellang hore okotsing e hodimo haholo. e kaba oe kutlwa joang ka tsebo e?

Kemeho ya hao ya kwatsi ya bosolla tlhapi entjha ha waka wa fumana nako e ngata ho nahana ka yona. Ho kaba boima ho nahana ka yona haholo hona jwale, empa ho bohlokwa ho qala ho nahana kaha hore otla ikuklwa o bolokehile le ratong la lona ka kameho ena.

Kena le tsebiso e bohlokwa bakeng sa hao. Basadi bana le ho bona thekefetso ka ditsela tse tharo: maikutlong, mmeleng le ka thobalano. Kaofela mefuta ena ya tlhekefetso e phahama kamora kameho ya HIV. Mehlala ya tlhekefetso ya maikutlo ao bitsa, baya fatshe kappa o leka ho itaola. Mehlala ya tlhekefetso ya mmele ya o hlola, yao raha, kappa ho o shapa. Mehlala ya tlhekefetso ya thobalano eo hatella ho robalana kappa ho o hatella ho etsa thobalano eo hatella ho robalana kappa ho o hatella ho etsa thobalano ntle le tshireletso.

Basadi ba kahara lerato hona le tlhekefetso kappa moruso,monyetla o moholo wa tlhekefetso haba qeta ho amohela sepheto sa HIV. Basadi ba bang ba tseba kotsi haba qeta ho thola kaha maemo a bona ho baratuwa ba bona kappa ba thola tsa tlhokomelo ka kalafo ya HIV.Motho ya hlekefetsa baka leka ho itshereletsa tekong ya thatlhobo kappa honka kalafo.

Hole jwalo ekaba nako e kotsi leratong la hao, basadi ba bangata ba tshepa ho bolella baratuwa ba bona ka maemo a bona hore baratuwa ba bona le bona ba hlahlojwe kappa bat hole kalafo. Habeba ban ka qeto ya ho bolella baratuwa ba bona. Ho bohlokwa hotla ka leano ho tshepahala ha seo ose etsang. Ke qeto ya hao, hao batla ho bolella moratuwa wa hao ka boemo ba hao ba HIV.

Ho lokile haeba o bolela hanyane ka ditsela tsa ho dula o bolokehile kahara lerato la hao?

22. Batho ba bangata bophelong ba hao le setjhabeng baka o thusang hao iketla ole kotsing ka hara lerato la hao. Sena seka konyeletsa ngaka ya hao, mooki, molemosi, baeteledi pele, baeteledi pele ba semoya kapa tshebeletso e itseng ya tlhekefetso ya thobalano.

$>$ Hona le maholale ditokomane tse itseng eo o ka e letsetsang hao hloka motho oo oka le yena kappa ya potlako. Nomoro ele new ke : 0800150-150. Ho bohlokwa ho ngola sena moo ho bolokehileng oka behang tsena? (mosebetsing wa hao kappa mohaleng wa hao omang le mabitso a fapaneng). (3) 
23. Batho ba bang ba thabela ho bua le kapa ba thole thuso bathong baba tsebang. Sena seka bolela ho bua le ba leloko la hao kapa metswalle e ka o thusa ha etsahala ho itseng .

> Nahana ka omong wa leloko kappa motswalle ba bolokehileng (yaka se hlahise sena ho moratuwa wa hao). 0 ka letsetsa motho eo ha fela hona leho potlakileng (sebedisa nomoro ya kgokahane bakeng sa thuso).

24. Hona le thuso ya molao bakeng sa moratuwa ya hlelefetsang. Mehlala, letsetsa maponesa, letsetsa mmuelli kappa otlatse diforomo tsa tshireletso.

$>$ Ho itshetlehile, hore foromo ya tshireletso etla thibela moratuwa wa hao kema ntlong ya hao kappa mosebetsing wa hao kappa hoba le kgokahano le bana ba hao. Tsena di thusa ho thibela diketso tsa tlhekefetso. 0 ka thabela dinomoro tse ka o thusang tsebang kaha tshireletso? (Di tshwanetse di phethahale hoya ka bopaki ba lefapha la seponesa) le hahole jwale kamtoro e kgolo (ko Petoria)ko+27 (0) 12 3931000 .

$>$ Boloka sena sebakang sa polokehe (ntlong yaw a leloko ho wena ntlong kappa sepatjheng tulong e kahare ka hara setlotsa molomo kappa ngola lebitso ka tshebetso e fapaneng haufi le yona (tokisong ya moriri,moo hontshwang dijo)

25. Batho ba bang ba leka ho qoba nakong ya kotsi ya tlhekefetso. Ha ntwa e bat eng batho ba bang ba leka ho kokobetsa ba ratuwa ba bona. Empa hoopla hore boitshwaro hase molato kappa boikarabelo.

> Hona le ka dinako tse ding moo oka nahanang hore o batla ho ema o hlwekile ka baka la bo ngata ba dikotsi? (4)

26. Batho ba bang ba leka ho balehela tlhekefetso ha e tsahala. Mohlala, ho leka ho ipuseletsa ho pata sethunya mo ho tsebang wena hore se kae ho leka ho tsamaya kappa ho fedisa lerato kappa hobaleya, hoipata nakong ya ketsahalo. Di patlisiso di bontshitse hore ho balehela tlhekefetso ho kotsi haholo ho eketsa le ntwa kappa moferefere.

$>$ O nahana ho tswa leratong? (5) Nnete tjhee, ha hole jwalo, ho lokile ho lohale qheka hobane ke nako e hlokolosi haholo nakong ya lerato lemoferefere. (6) Leqheka ke

27. Mekgahlelo emeng e kenyeletsa ho lokisetsa botlako le ho pata tjhelete, ho bohlokwa ho letsetsa dinomoro le batho haeba o hloka ho tsamaya ka potloko. Hape ho holokile ho ba leleqheka pele, o kaya ntlong ya hao ha ntwa eka etsahala (eya kamoreng e bolokehileng moo ho semang dibetsa kapa dintho tse bohale)

Hare kwala pitso ena, re lakatsa ho o thusa ho etsa leqheka hore otla itlhokomela ka bowena, o fane ka botjha ba kameho le kotsi leratong.

Hao nka qeto ya ho bolella moratuwa wa hao ka boemo ba hao, hona jwale kappa pele letsatsi le dikela, ke ditsela tse feng tseo oka itshereletsang ka tsona? (7) 
Hao belaela hore moratuwa wa hao o etsa thobalano le motho omong, ho bohlokwa ho tseba hoo, o mo bolelle kaha sona. Sena seka hohela moferefere o moholo.

Ho sebedisa tshireletso ka mora hoba o thole tshwaetso ya HIV, ho eketsa bontshitse ho eketseha ha tshwaetso empa ho kopa moratuwa wa hao ho sebedisa tshireletso hoka bontsha ho eketsa moferefere. Empa hao qla ho sebedisa tshireletso, nahana tsela ya polokeho ya ho bua le moratuwa wa hao ka yona.

Hotlang pele ka mora kameho hanghang eya kalafong ho thola pheko ya meriana.

Ho fihlela onka qeto hore o batla ho bolella moratuwa wa hao ka kameho, o tla thola jwang tshebeletso ya pheko.

Ohloka honka qeto ya ho bolella moratuwa wa hao hore hona le koo oyang teng? 0 tlareng(8)

Haeba CD4 cell count (ele tlase )e tla totobatswa hore o qale ka ho nka kalafi e tshwanetseng. Mokga o bolokehileng o tlwaelehileng ke ho pata di kalafo tsa hao ho moratuwa wa hao ya o hlekefetsang. Ke ditsela tse feng tseo o ka di etsang (mohlala, ho Kenya dipidisi ka hara lebotlolo la polasitiki kappa le boloke le wena). 0 ka etsang?(9)

Hona le seo reka ka o thusang ka sona kajeno hotleo bolokehe ho fumana pheko eo oe hlokang hape itlhokomele mmeleng le moyeng?

(10)

Appendix W

Post-test Experimental group Sesotho

Tlhatlhobo Post-test Date ___ Time of test 


\begin{tabular}{|c|c|c|}
\hline \multicolumn{3}{|l|}{ 1. HIRS } \\
\hline \multicolumn{3}{|c|}{$\begin{array}{l}\text { Ha esale o a amohela sepheto sakwatsi ea bosolla thapi ...ekaba o dumellana le } \\
\text { se bolelwang? }\end{array}$} \\
\hline 1. 0 bile ka hara mathata le moratuwa wa hao & Nnete & \begin{tabular}{l|l} 
Tjhe \\
$\mathrm{e}$
\end{tabular} \\
\hline 2. Moratuwa wa hao o bile tshehetso ho wena & Nnete & $\begin{array}{l}\text { Tjhe } \\
\text { e }\end{array}$ \\
\hline 3. Setswalle sa lona setlaba matateng hofitisisa & Nnete & $\begin{array}{l}\text { Tjhe } \\
\text { e }\end{array}$ \\
\hline $\begin{array}{l}\text { 4. Ho thola kalafo hobi le kotsi ka baka la moratuwa wa } \\
\text { wahao }\end{array}$ & Nnete & $\begin{array}{l}\text { Tjhe } \\
\text { e }\end{array}$ \\
\hline 5. 0 ikutlwa osa bolokeha setswalleng la lona & Nnete & $\begin{array}{l}\text { Tjhe } \\
\text { e }\end{array}$ \\
\hline $\begin{array}{l}\text { 6. Ba bang ba tholahala hoo thusa hao ikutlwa osa bolokeha } \\
\text { setswalleng la lona }\end{array}$ & Nnete & $\begin{array}{l}\text { Tjhe } \\
\text { e }\end{array}$ \\
\hline $\begin{array}{l}\text { 7. Hone hosa bolokeha ho bua ka teko ya kwatsi ya bosolla } \\
\text { thapI }\{\text { HIV }\} \text { le moratuwa wa hao }\end{array}$ & Nnete & $\begin{array}{l}\text { Tjhe } \\
\text { e }\end{array}$ \\
\hline $\begin{array}{l}\text { 8. Hone hosa bolokeha ho bua ka boemo ba hao ba HIV le } \\
\text { moratuwa wa hao }\end{array}$ & Nnete & $\begin{array}{l}\text { Tjhe } \\
\text { e }\end{array}$ \\
\hline 9. 0 ikutlwile o bolokehile ho kena hara hlophiso ya maphelo & Nnete & $\begin{array}{l}\text { Tjhe } \\
\text { e }\end{array}$ \\
\hline $\begin{array}{l}\text { 10. oile waikutlwa otsoha hore molekane ao hao aka leka ho o } \\
\text { bolaya }\end{array}$ & Nnete & $\begin{array}{l}\text { Tjhe } \\
\text { e }\end{array}$ \\
\hline \multicolumn{3}{|l|}{$2 . S S$} \\
\hline \multicolumn{3}{|l|}{ O dumellana le sebolelwang: } \\
\hline $\begin{array}{l}\text { 1. Kaofela thuso ena eo oye fumaneng ka tsa setswalle ebile } \\
\text { molemo }\end{array}$ & $\begin{array}{l}\text { Nnet } \\
\text { e }\end{array}$ & Tjhee \\
\hline 2. Ho bile thata ho botswa katsa marato a hao & $\begin{array}{l}\text { Nnet } \\
\text { e }\end{array}$ & Tjhee \\
\hline $\begin{array}{l}\text { 3. O lakatsa hore nkabe o buile ka mathatha a hao a setswalle } \\
\text { hofeta }\end{array}$ & $\begin{array}{l}\text { Nnet } \\
\text { e }\end{array}$ & Tjhee \\
\hline $\begin{array}{l}4.0 \text { ne osa phothuloha hao ne o bua ka mathato a tsa setswalle sa } \\
\text { hao }\end{array}$ & $\begin{array}{l}\text { Nnet } \\
\text { e }\end{array}$ & Tjhee \\
\hline 5. Wa itshola ho bua ka mathata a setswalle sa hao & $\begin{array}{l}\text { Nnet } \\
\text { e }\end{array}$ & Tjhee \\
\hline $\begin{array}{l}\text { 6. Ho bua ka mathata setswalleng la hao ho o beile mongetleng o } \\
\text { moholo wa kotsi }\end{array}$ & $\begin{array}{l}\text { Nnet } \\
\text { e }\end{array}$ & Tjhee \\
\hline $\begin{array}{l}\text { 7. Tshebeletso eo o efumanang eo beile mnyetleng o moholo wa } \\
\text { kotsi }\end{array}$ & $\begin{array}{l}\text { Nnet } \\
\text { e }\end{array}$ & Tjhee \\
\hline \multicolumn{3}{|l|}{ 3.FAS } \\
\hline 1. O sebedisitse leqheka le bolokehileng? & Tjhe & \\
\hline
\end{tabular}




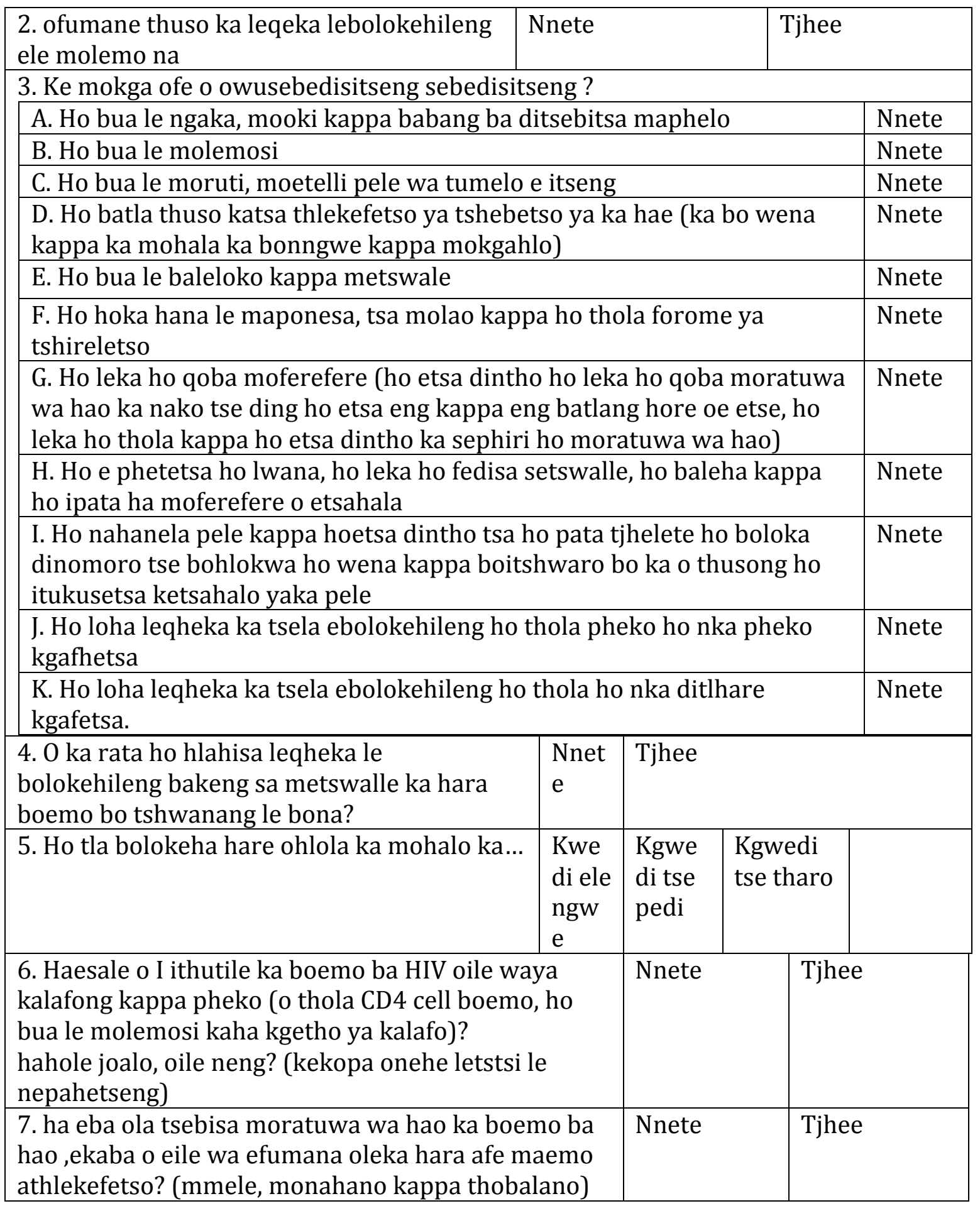

Time of test end 
Appendix X

Post-test Standard of Care Sesotho

\begin{tabular}{|c|c|c|}
\hline \multicolumn{3}{|l|}{ Post-test Date $\quad$ Time of test } \\
\hline \multicolumn{3}{|l|}{ 1. HIRS } \\
\hline \multicolumn{3}{|c|}{$\begin{array}{l}\text { Ha esale o a amohela sepheto sakwatsi ea bosolla tlhapi ...ekaba o dumellana } \\
\text { le se bolelwang? }\end{array}$} \\
\hline 1. 0 bile ka hara mathata le moratuwa wa hao & Nnete & \begin{tabular}{l|l} 
Tjhee \\
\end{tabular} \\
\hline 2. Moratuwa wa hao o bile tshehetso ho wena & Nnete & Tjhee \\
\hline 3. Setswalle sa lona setlaba matateng hofitisisa & Nnete & Tjhee \\
\hline $\begin{array}{l}\text { 4. Ho thola kalafo hobi le kotsi ka baka la moratuwa wa } \\
\text { wahao }\end{array}$ & Nnete & Tjhee \\
\hline 5. 0 ikutlwa osa bolokeha setswalleng la lona & Nnete & Tjhee \\
\hline $\begin{array}{l}\text { 6. Ba bang ba tholahala hoo thusa hao ikutlwa osa } \\
\text { bolokeha setswalleng la lona }\end{array}$ & Nnete & Tjhee \\
\hline $\begin{array}{l}\text { 7. Hone hosa bolokeha ho bua ka teko ya kwatsi ya bosolla } \\
\text { thapI }\{\text { HIV }\} \text { le moratuwa wa hao }\end{array}$ & Nnete & Tjhee \\
\hline $\begin{array}{l}\text { 8. Hone hosa bolokeha ho bua ka boemo ba hao ba HIV le } \\
\text { moratuwa wa hao }\end{array}$ & Nnete & Tjhee \\
\hline $\begin{array}{l}\text { 9. } 0 \text { ikutlwile o bolokehile ho kena hara hlophiso ya } \\
\text { maphelo }\end{array}$ & Nnete & Tjhee \\
\hline $\begin{array}{l}\text { 10. oile waikutlwa otsoha hore molekane ao hao aka leka } \\
\text { ho o bolaya }\end{array}$ & Nnete & Tjhee \\
\hline \multicolumn{3}{|l|}{ 2.SS } \\
\hline \multicolumn{3}{|l|}{ O dumellana le sebolelwang: } \\
\hline $\begin{array}{l}\text { 1. Kaofela thuso ena eo oye fumaneng ka tsa setswalle ebile } \\
\text { molemo }\end{array}$ & Nnete & Tjhee \\
\hline 2. Ho bile thata ho botswa katsa marato a hao & Nnete & Tjhee \\
\hline $\begin{array}{l}\text { 3. O lakatsa hore nkabe o buile ka mathatha a hao a setswalle } \\
\text { hofeta }\end{array}$ & Nnete & Tjhee \\
\hline $\begin{array}{l}\text { 4. } 0 \text { ne osa phothuloha hao ne o bua ka mathato a tsa setswalle } \\
\text { sa hao }\end{array}$ & \begin{tabular}{l|l} 
lle & Nnete \\
\end{tabular} & Tjhee \\
\hline 5. Wa itshola ho bua ka mathata a setswalle sa hao & Nnete & Tjhee \\
\hline $\begin{array}{l}\text { 6. Ho bua ka mathata setswalleng la hao ho o beile mongetleng } \\
\text { o moholo wa kotsi }\end{array}$ & Nnete & Tjhee \\
\hline $\begin{array}{l}\text { 7. Tshebeletso eo o efumanang eo beile mnyetleng o moholo } \\
\text { wa kotsi }\end{array}$ & Nnete & Tjhee \\
\hline
\end{tabular}




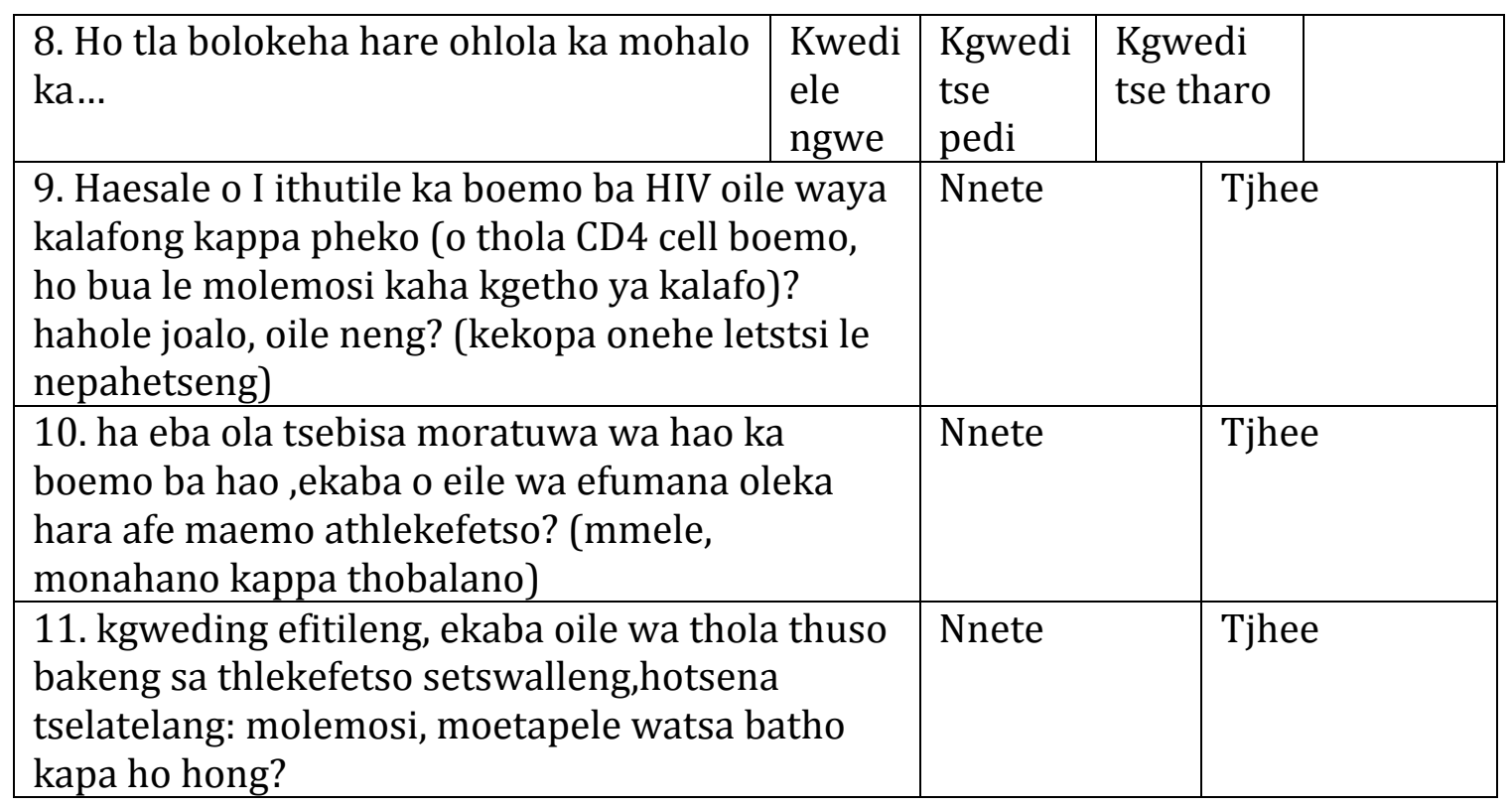




\section{CURRICULUM VITAE}

Lauren Brown, LCSW, MSSW

Kent School of Social Work

University of Louisville, Louisville, KY

Julius John Oppenheimer Hall

School of Social Work

2217 S $3^{\text {rd }}$ St.

Louisville, KY 40292

(615) 557-3499

lauren.brown@louisville.edu

\section{EDUCATION}

$\mathrm{PhD}$

University of Louisville

Kent School of Social Work

Expected May 2016

LCSW

Board of Health, Tennessee

2012-present

MSSW

University of Tennessee, Nashville

School of Social Work

Clinical Track

August 2008

BA

Middle Tennessee State University

English (Minor in Classical Studies)

2003

\section{DISSERTATION}

Title: Mitigating Intimate Partner Violence to Promote Safe Linkage to Care for South African Women who test HIV+ During Mobile HIV Counseling and Testing

Committee: Michiel "Riaan" van Zyl (Chair), Bibhuti Sar, Anita Barbee, Marie Antoinette Sossou, \& Emma Sterrett-Hong

- Phase II trial study using an experimental pre post design, with two tests groups of standard of care and experimental and two treatment arms 
within experimental, to test effectiveness and safety of HIV-IPV risk and safety protocol

- Creation of a reliable psychometric instrument (Cronbach's alpha coefficient .882) measuring risk and safety relative to the HIV-IPV intersection

- Chi-square and Independent sample t tests were conducted to tests for condition differences on safety scores, linkage to care rates, and post-test satisfaction, feasibility and acceptability score differences among cohorts.

\section{RESEARCH INTERESTS}

- Intimate Partner Violence

- Trauma

- HIVIAIDS

- HIV and IPV link

- International Social Work and Global Health

- Social Work Epistemology

- Forensic Social Work

- Older Adults

- Scale development in social work

\section{TEACHING INTERESTS}

- Trauma

- Direct Social Work Practice

- Psychopathology

- International Social Work

- Macro Practice

- Anti-Oppressive Social Work

- Research Methods

\section{GRANTS \& ASSISTANTSHIPS}




\begin{tabular}{|c|c|c|}
\hline 2015 & $\$ 3000$ & $\begin{array}{l}\text { Dissertation Research Grant } \\
\text { Kent School of Social Work }\end{array}$ \\
\hline 2015 & $\$ 3000$ & $\begin{array}{l}\text { Dissertation funding by committee member } \\
\text { Kent School of Social Work }\end{array}$ \\
\hline 2015 & $\$ 1000$ & $\begin{array}{l}\text { Dissertation funding by Assistant Dean of } \\
\text { Research } \\
\text { Kent School of Social Work }\end{array}$ \\
\hline 2013 & Admission & $\begin{array}{l}\text { IDEA festival scholarship } \\
\text { Louisville, KY }\end{array}$ \\
\hline 2013 & $\$ 600$ & $\begin{array}{l}6^{\text {th }} \text { annual SAAIDS conference scholarship } \\
\text { SAAIDS, Durban, South Africa }\end{array}$ \\
\hline 2013 & $\$ 2000$ & $\begin{array}{l}\text { Dean's Office Award for Conference Travel } \\
\text { Kent School of Social Work }\end{array}$ \\
\hline 2012-2014 & $\$ 38,000$ & $\begin{array}{l}\text { Doctoral Student Assistantship } \\
\text { University of Louisville }\end{array}$ \\
\hline \multicolumn{3}{|c|}{ Other Grant Experiences } \\
\hline 2014 & $\begin{array}{l}\text { submitted, } \\
\text { not funded }\end{array}$ & $\begin{array}{l}\text { National Institute of Health RO1 } \\
\text { University of Louisville, University of California } \\
\text { Los Angeles, Shout-it-Now } \\
\text { Assisted with development of application } \\
\text { largelyby creating literature review. }\end{array}$ \\
\hline
\end{tabular}

\section{PUBLICATIONS}

Peer-reviewed journal articles

1. Norton, L., Woods, G., Brown, L. (2014). Secondary Trauma in Forensic Settings: Effects on Court Personnel, Jurists, Jurors, and Correctional Officers. International Journal of Trauma Research and Practice, 1(1), 2833.

2. Van Zyl, A.M., Brown, L.L. Pahl, K. (2015). Using a Call Center to Expand Mobile HCT and Link HIV positive Individuals to Care. AIDS Care. Print information forthcoming. (2014 Impact Factor 2.095) 
3. Brown, L.L. (under review). HIV IPV Risk \& Safety Protocol for Young South African Women Post Mobile HIV Counseling and Testing.

\section{In preparation}

1. Brown, L.L., van Zyl, A.M., Pahl, K. Attitudes of Gender Inequalities among Mobile HIV Testers (in preparation).

2. Held, M.L., Brown, L.L. (in preparation). Effects of Intimate Partner Violence on the Well-Being of Hispanic Women and their Children.

3. Cooper, R.L., Brown, L., Cummings, S. (in preparation). Outcomes of a pilot study of a combined MI \& CBT intervention for older adult public housing residents.

4. Brown, L., Winter, G. (in preparation). Social Work \& Capital Punishment: A call to action in Tennessee

5. Brown, L. Boamah, D., Thompson, J., van Zyl, A. The Practice of Social Work Knowledge Building: Beyond Postmodernism. (in preparation).

\section{Technical reports:}

Van Zyl, M.A., Pahl, K, Brown, L.L. (2013). Youth attitudes, perceptions and behaviors around gender norms and gender based violence in South Africa. Report submitted to Swedish International Development Cooperation, December 2013.

\section{PRESENTATIONS}

Brown, L.L. \& Lawson, J. (2015). Active Teaching to Promote Collaborative Engagement in Online Learning Communities. Break out presentation at Council on Social Work Education annual conference in Denver, CO, October, 2015.

Cooper, R.L., Cummings, S., Brown, L.L. (2014). Substance Abuse and Mental Health Among Older Adults in Public Housing. Paper presentation at Council on Social Work Education, annual conference in Tampa, FL, October 2014.

Faul, A.C., Gordon, B., D'Ambrosio, J.G., Ferrell, G., Tucker, M., Schapmire, T.J., Fields, M., Thompson, J., Rhema, S., Miller, J.D., Boamah, D.A., Brown, L., Boes, R.C., Zheng, Y., Lawson, T.R. (2013). Academia and the 
Aging Network. 38th Annual N4A Conference and Tradeshow, Louisville, KY, July $27-31$.

Faul, A.C., Lawson, T. R., D’Ambrosio, J.G., Boamah, D.A., Cotton, S., Alkohaiz, M., Smith, L.D., Lewis, S.N., \& Brown, L. (2014). Collaborative Teaching and Learning as a Tool to Ignite the Spark in students. 2014 Celebration of Teaching and Learning, University of Louisville, Delphi Center for Teaching \& Learning, Louisville, KY, February 7.

Faul, A.C., Lawson, T. R., D’Ambrosio, J.G., Boamah, D.A., Brown, L., Fields, M. (2014). Collaborative Teaching and Learning as a Tool to Ignite the Spark in students. 2014 Kentucky Association of Social Work Educators. Western Kentucky University, Bowling Green, KY, April 10.

Faul, A.C., Gordon, T.R. Lawson, Boamah, D.A., Boes, R.C., Brown, L.L., J.G., Fields, M., Thompson, J., Zheng, Y., (2013). Lower Income, Vulnerable Adults: Effects of Nutritional Programs on Aging in Place. Summer Series on Aging, Louisville, KY June.

Van Zyl, R.M., Pahl, K., Brown, L.L. (2013) Innovations in Links to Care for Community-based HCT Programmes, SAAIDS Conference, Durban, South Africa, June 2013

Brown, L.L. Domestic Violence Resources and Emotional Safety Planning. Nashville Coalition Against Domestic Violence (NCADV) Annual Training. Nashville, TN, 2011.

Brown, L.L., Kennedy, B. Domestic Violence Shelter Rules: Rising to the Changes in our Movement: advocating without power and control. Tennessee Coalition Against Domestic and Sexual Violence (TCADSV) Annual Conference. Franklin, TN, 2009.

Brown, L.L. Butler, A. Domestic Violence through the Lens of Family Systems Theory. Tennessee Coalition Against Domestic and Sexual Violence (TCADSV) annual conference. Franklin, TN, 2007

\section{RESEARCH EXPERIENCE}

$2015 \quad$ Research Assistant, Qualitative Coder

Principal Investigator: Mary Lehman Held

University of Tennessee

Hired as researcher, with knowledge of Latin American culture, to assist with coding qualitative component of mixed methods 
dissertation study over remunerations with immigrant populations. Created themes and codes over focus groups and interviews and then discussed themes and codes with other coder to enhance the study's validity and reliability.

2015 Co-Investigator, Fragile Families

Principal Investigator: Mary Lehman Held

University of Louisville \& University of Tennessee

Used secondary data from the Fragile Families data set to build knowledge on the effects of Intimate Partner Violence (IPV) on Hispanic/Latina women and their children. Applied logistic regression to analyze how the differences between country nativity and contextualized intimate partner violence (IPV) affect mental and physical well-being of women and their children. Conducted literature review, assisted with manuscript composition and variable coding.

2014- Co-Investigator, Tennesseans for Alternatives to the Death present penalty Principal Investigator: Michiel van Zyl Nashville, TN

Collaborated with community organization to analyze secondary data over three years of student conference evaluations. Used basic descriptive statistics to analyze demographic information of student attendees. Conducted literature review, composed manuscript, analyzed results, and collaborated with MSSW student intern.

2014- Co-Investigator, The Family Center

present Nashville, TN

Principal Investigator: Anita Barbee

Collaborated with community organization to analyze secondary data over 12 months of pre/post surveys used to measure the efficacy of parenting classes administered in prisons, community centers and drug and alcohol programs. Will use Hierarchal Linear Modeling to analyze efficacy of programs given the various site locations. Used Confirmatory Factor Analysis (CFA) to test validity and reliability of psychometric tool (Adult Adolescent Parenting Inventory version-2).

2014 Graduate Student Researcher, Kent School of Social Work Principal Investigator: Michiel van Zyl University of Louisville 
Analyzed results from Kent School of Social Work's students' pre/post responses on the California Critical Thinking Survey, and assisted in the development of discipline-specific survey for social work. Built on previous cohort's social work critical thinking survey and used Classical Test Theory and Item Response Theory to test old and new items to replace those with unacceptable reliability and validity.

2013-2014 Graduate Student Researcher, Shout-it-Now

Principal Investigator: Michiel van Zyl

University of Louisville and Gauteng \& Limpopo provinces, South

Africa

Under a grant from the Swedish government, conducted study to determine link between gender inequalities and HIV in youth in South African provinces of Gauteng and Limpopo. Used principle component analysis (PCA) for scale development of psychometric tool created to measure violence vulnerability of participants. After PCA, Cronbach alpha of created subscale was .807 with a Corrected Mean Total Correlation of .45.

2013- Graduate Student Researcher, Center for Women and Families present Principal Investigator: Anita Barbee University of Louisville and Louisville, $K Y$ Collaborated with community agency for chart review (secondary data analysis) to help organization conduct program evaluation. Acted as consultant to organization in their transition to a trauma informed center or program of No Rules.

2013-2015 Graduate Student Researcher, Shout-it-Now Principal Investigator: Michiel van Zyl University of Louisville \& Gauteng and Limpopo provinces, South Africa

Secondary data analysis over data collected by agency's call centre. Use of Chi Square Automatic Interaction Detection (CHAID) analysis to determine success of links-to-care program. Conducted literature review, prepared manuscript, submitted and was approved by IRB, submitted to journal and was published.

2012-2014 Graduate Student Researcher, Kentuckiana Regional Planning \& Development Agency (KIPDA)

Principal Investigators Anna Faul and Tom Lawson

University of Louisville

Conducted collaborative study between Kent school of social work doctoral program and KIPDA to help determine efficacy of three 
different KIPDA programs on older adults aging in place. Presented results and won poster award at Summer Series on Aging.

2011- Project Coordinator, Metropolitan Development \& Housing Agency 2014 (MDHA)

Principal Investigator: Robert Cooper

Oversaw and administered pilot research study done in collaboration between MDHA and University of Tennessee.

Administered study as clinician, oversaw grant reporting and later assisted with data analysis. Used Fischer's exact test and paired sample t tests during analysis.

\section{TEACHING EXPERIENCE}

Fall, 2014 Instructor

\& 2015 Advanced Social Work Practice II (SW 640 Distance Education) University of Louisville, Louisville, KY

This is an online course that utilizes Blackboard, Softchalk, Tegrity and Collaborate for virtual meetings. This course teaches advanced problem-solving practice models and critical thinking skills to assist social workers in preparation for clinical roles or supervision of direct service providers. Practice models taught are crisis intervention theory, solution-focused therapy, motivational interviewing, and cognitive-behavioral therapy. The course requires integration of Advanced Research Course material related to: evidence-based practice, evaluation, critical thinking, problem solving, and planned intervention.

\section{Spring, 2015 Instructor}

Advanced Social Work Practice III (SW 677 Distance

Education)

University of Louisville, Louisville, KY

This course provides special attention to complex practice situations encountered by social workers in social welfare organizations, schools, health and mental health care facilities, and in the community. Students learn intervention strategies and practice skills to promote change at multiple levels of social systems using a case based learning approach. The first half of the course explores trauma and trauma-focused care, and the latter half focuses on the theory and application of Anti-Oppressive social work practice on multi-systemic levels. The course helps students bridge clinical and macro-level social work and seeks to inspire 
them to practice and respond to oppression on multi-levels as they commit to lifelong learning.

Spring, 2015 Course Designer

Advanced Social Work Practice III (SW 677) Distance Education Designed online course for entire online course sequence and was asked by Associate Dean to do so as the course had never been taught online. Created modules for weekly sessions using Softchalk and designed assignments to fit online medium.

Fall, 2014 Course Designer

Advanced Social Work Practice III (SW 677) Distance Education Designed online course for entire online course sequence and was asked by Associate Dean for Academic Affairs to do so as the course had never been taught online. Created modules for weekly sessions using Softchalk and designed assignments to fit online medium.

Spring, 2014 Instructor

Advanced Social Work Practice III (SW 677)

University of Louisville, Louisville, KY

This is the same course as listed above but is taught face-to-face within a traditional classroom setting.

Fall, 2013 Graduate Instructor

Advanced Social Work Practice II (SW 640)

University of Louisville, Louisville, KY

This is the same course as listed above but is taught

face-to-face within a traditional classroom setting.

Jan., 2009- Instructor

Dec. 2011 Social Work Interviewing Skills (SW 220)

Nashville State Community College

Nashville, TN

This course teaches basic social work interviewing skills focusing on engagement, assessment, the helping phase, evaluation and termination. Students learn basic principles of ethical decisionmaking and how to use an ethics hierarchy to resolve ethical dilemmas. Basic practice skills are also taught.

\section{SOCIAL WORK PRACTICE EXPERIENCE}

\section{Mar., 2015- Mitigation Expert}


Present Contract consultation

Provide social work mitigation for homicide cases with public

defender's office

Jan., 2014- Mitigation Expert

Present Center for Trauma Therapy

Chronologist for capital homicide cases

Sep., 2012- Psychotherapist

Present Symmetry Counseling

Focusing on trauma (sexual assault, etc.), transgender issues, \& self harm. Work with teens, young adults, adults, couples \& families. Use of EMDR, Cognitive Behavioral Therapy, Motivational Interviewing and Acceptance and Commitment therapy

Sep. 2011- HeLP Project Coordinator

Apr., 2014 Metropolitan Development \& Housing Agency (MDHA)

Administered research study using Motivational Interviewing and Cognitive Behavioral therapy for older adults with substance misuse, mental health or dual diagnosis; Managed grant and implemented pilot study.

Sep., 2007- Program Coordinator

Sep. 2011 YWCA Domestic Violence Center

Supervised full-time and part-time crisis counselors, case managers, childcare workers and interns in residential and hotline setting

Oct., 2008- Therapist

Sep., 2011 YWCA Domestic Violence Center Conducted individual and group therapy using Cognitive Behavioral Therapy and Motivational Interviewing

Aug., 2007- MSSW Field Practicum

Jul., 2008 Nashville Cares, HIVIAIDS

Individual and group therapy with adults infected and affected with HIVIAIDS who either had mental health issues or substance abuse Worked in Intensive Outpatient treatment

Aug., 2006- MSSW Foundation Field Practicum

May, 2007 YW Newstart Program, TN Prison for Women, Individual and group therapy for incarcerated women in minimum security 
Mar., 2005- Children's Counselor

Sep., 2007 YWCA Domestic Violence Center

Individual, family and group sessions in-shelter and in community

with DeDe Wallace, Oasis center and Monroe Harding

Conducted Parenting group in-shelter and TN Prison for Women

Child Abuse Review Team with Child Protective Services

Mar., 2004- Women's Advocate

March, 2005 YWCA Domestic Violence Center

Answered hotline, conducted intakes, worked in residential setting

\section{CERTIFICATES \& SKILLS}

2012- $\quad$ CITI and HIPPA certified

present

2012- Licensed Clinical Social Worker

present

2011/2012 EMDR Level II trained

2008 Phi Alpha Honor Society lota Epsilon Chapter, 4.0 GPA University of Tennessee

2009-2011 Spanish courses, Nashville State Community College

2012-2014 Spanish courses, Tennessee Foreign Language Institute Conversational Spanish and extensive travel in Latin America:

Colombia, Dominican Republic, Puerto Rico, Argentina, Uruguay, Paraguay, Peru, Bolivia, Brazil, Mexico, Costa Rica, Panama, Belize, and Guatemala.

\section{SERVICE SOCIAL WORK PROFESSION}

Fall, 2015 Peer Reviewer for AIDS Care

Invited personally by editors of UK-based Taylor \& Frances journal, with impact factor of 2.095, for specific article as an expert on HIV services in South Africa

Feb. 2009- Board of Directors 
Present Tennesseans for Alternatives to the Death Penalty Lobby for death penalty repeal; fundraise for agency operation; organize and manage annual benefit; oversee organization; mobilize community towards abolition

Apr., 2013 Capstone Judge

BSW Capstone poster presentations

Oct. 2009- Board of Directors

Oct. 2012 South Nashville Action People

Organize neighborhood events such as monthly meeting, Creek clean up and holiday gift drive; help re-write organization mission and by laws; worked on grants to move from volunteer-based organization to one with two paid staff; oversaw budget of organization as well as organization-owned affordable housing units; oversaw renovation of housing units and assisted with money appropriation of housing revenue.

2009-2012 Board of Directors, Chair 2009-2010, Co-Chair 2011-2012 Nashville Coalition Against Domestic Violence, Conducted monthly meetings, organized events such as Meet Us at the Bridge, NCADV annual legal training and NCADV annual benefit; synergized with domestic violence service providers in Nashville to provide cohesive and unified response to domestic violence in Davidson county; wrote and received a grant to provide financial assistance to community victims of domestic violence and then oversaw scholarship of grants on case-by-case basis.

\section{REFERENCES}

\section{Riaan van Zyl}

Professor, Associate Dean of Research

Kent School of Social Work

109 Oppenheimer Hall

University of Louisville

Louisville, $\mathrm{KY}$

1-502-852-2430

Mavanz01@louisville.edu

\section{Anita Barbee}

Professor \& Distinguished University Scholar

Kent School of Social Work

202 Oppenheimer Hall 
University of Louisville

Louisville, $\mathrm{KY}$

1-502-852-0416

Anita.barbee@louisville.edu

Bibhuti Sar

Professor \& Doctoral Program Director

Kent School of Social Work

Patterson Hall

University of Louisville

Louisville, KY

1-502-852-3932

b.k.sar@louisville.edu

Anna Faul

Professor, Associate Dean for Academic Affairs

Kent School of Social Work

Oppenheimer Hall

University of Louisville

Louisville, KY

1-502-930-5445

Acfaul01@louisville.edu

Sandra Gonzalez

Instructor

Baylor College of Medicine

Department of Family and Community Medicine

3701 Kirby Dr., Suite 600

Houston, TX 77098

Sandra.gonzalez@bcm.edu

1-713-798-3633 